NISTIR 7953

\title{
Interlaboratory Analytical Comparison Study of Total Fatty Acid Concentrations in Human Serum: Results for Exercise 01: QA12FASER01
}

\author{
Michele M. Schantz \\ Carissa D. Powers \\ Rosemary L. Schleicher
}


NISTIR 7953

\section{Interlaboratory Analytical Comparison Study of Total Fatty Acid Concentrations in Human Serum: Results for Exercise 01: QA12FASER01}

Michele M. Schantz

Chemical Sciences Division Material Measurement Laboratory

Carissa D. Powers and Rosemary L. Schleicher Centers for Disease Control and Prevention (CDC) Atlanta, GA

http://dx.doi.org/10.6028/NIST.IR.7953

September 2013

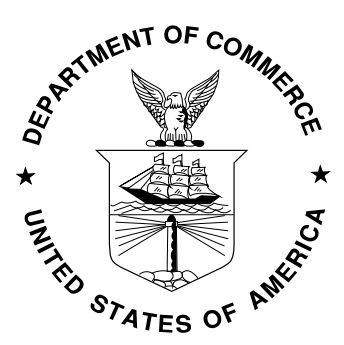

U.S. Department of Commerce Penny Pritzker, Secretary

National Institute of Standards and Technology Patrick D. Gallagher, Deputy Secretary of Commerce and Director 


\section{ABSTRACT}

The National Institute of Standards and Technology (NIST), in conjunction with the Centers for Disease Control and Prevention (CDC) and NIH's Office of Dietary Supplements (ODS), initiated an interlaboratory analytical comparison study of total fatty acid concentrations in human serum in May 2012. The first sample was candidate Standard Reference Material (SRM) 2378. SRM 2378 consists of three levels of fatty acids in serum collected from: (1) donors who have not taken fish or flaxseed oil supplements for one month prior to collection; (2) donors who have taken flaxseed oil supplements for a minimum of one month prior to collection; and (3) donors who have taken fish oil supplements for a minimum of one month prior to collection. In addition, participants were requested to run SRM 1950 Metabolites in Human Plasma as a control sample. This is a performance-based program so participating laboratories were requested to use the analytical procedures that they typically use in their laboratories for these analyses and report data for those fatty acids that they typically quantify. The results from the first exercise are reported along with a summary of the analytical methods used. 


\section{Overview of Exercise 01: QA12FASER01}

For the first exercise of Interlaboratory Analytical Comparison Study of Total Fatty Acid Concentrations in Human Serum, candidate Standard Reference Material (SRM) 2378 was distributed as the study sample along with SRM 1950 Metabolites in Human Plasma which was distributed as the control sample. Candidate SRM 2378 consists of three serum materials collected from: donors who have not taken fish or flaxseed oil supplements for one month prior to collection; donors who have taken flaxseed oil supplements for a minimum of one month prior to collection; and donors who have taken fish oil supplements for a minimum of one month prior to collection. SRM 1950 is designed to represent "normal" human plasma. Plasma was obtained from 100 individuals (equal number of men and women in a narrow age range (40 to 50 years) who had undergone an overnight fast prior to blood draw. Certified and reference values are provided for selected fatty acids on the Certificate of Analysis for SRM 1950(https://www-s.nist.gov/srmors/view detail.cfm?srm=1950).

The laboratories were provided with four vials of each material and were requested to do triplicate measurements for each material (SRM 1950 and three levels of SRM 2378) using their laboratory's and/or program's analytical protocols, for the concentrations of the fatty acids currently being determined in their laboratory. A target list of compounds is presented in Table 1; however, participants did not need to quantify all of these compounds and could add additional compounds when reporting data. They were requested to report results, using three significant figures, in triplicate per analyte for each level of candidate SRM 2378 and for SRM 1950 in units of either $\mu \mathrm{g} / \mathrm{g}$ or $\mu \mathrm{mol} / \mathrm{L}$ and to provide brief descriptions of their cleanup and analytical procedures.

\section{REPORTED RESULTS}

Laboratories were assigned numerical identification codes in order of receipt of data with the exception of the NIST laboratory which is Lab 1 in this exercise. The laboratory mean replicate data are shown in Tables 2 to 5 for SRM 2378-1, SRM 2378-2, SRM 2378-3, and SRM 1950, respectively. Included in these tables are the laboratory mean values, standard deviations, and relative standard deviations both in the units that were reported and in a common unit, $\mu \mathrm{mol} / \mathrm{L}$. Summaries of the methods used by each laboratory are in Appendix A as well as charts of the mean numerical results reported by each laboratory for each analyte in the exercise materials.

\section{DISCUSSION}

NIST, CDC, and NIH's ODS contacted over 30 laboratories in early 2012 regarding participation in an interlaboratory analytical comparison study of total fatty acid concentrations in human serum. Of the laboratories contacted, 13 replied with interest in participating in this study and received samples in May 2012. The deadline for receipt of data was July 31, 2102. At that point, however, only seven laboratories had returned data so the deadline was extended with four additional laboratories submitting data. An alphabetical list of laboratories who returned data is given in Appendix B. Please note that the numerical codes in the following tables and figures are based on the receipt of the data and therefore do not follow the order in Appendix B. 
Laboratories were requested to submit data for the fatty acids that they typically monitor in similar samples (suggested list in Table 1) for three subsamples of each sample received (SRM 1950 and three levels of candidate SRM 2378) in units of either $\mu \mathrm{g} / \mathrm{g}$ or $\mu \mathrm{mol} / \mathrm{L}$ along with the density of each sample. Summaries of the results submitted by each laboratory are presented in Tables 2 through 5 for SRM 2378-1, SRM 2378-2, SRM 2378-3, and SRM 1950, respectively. In Table 5, the certified and reference values for SRM 1950 are also summarized for comparison. The data from each laboratory are presented in the tables as the means, standard deviations, and relative standard deviations for the three values submitted. Four laboratories reported the data in $\mu \mathrm{g} / \mathrm{g}$ (shown in red in Tables 2 through 5). These data were converted to $\mu \mathrm{mol} / \mathrm{L}$ using the densities provided by each laboratory except for lab 9 . Lab 9 did not report a density so the density was assumed to be $1 \mathrm{~g} / \mathrm{mL}$ for the conversion to $\mu \mathrm{mol} / \mathrm{L}$.

The relative standard deviations (RSDs) for the laboratory data are generally $<20 \%$. Lab 3 had a few analytes with higher RSDs (up to $41 \%$ ) as did lab 4 (up to $36 \%$ ), lab 7 (up to $60 \%$ ), lab 8 (up to $31 \%$ ), and lab 9 (up to $27 \%$ ), but these were generally based on a limited number of analytes in each sample. Labs 3 and 4 reported data for three fatty acids not included in Table 1, Lab 7 reported data for nine additional fatty acids, and Lab 11 reported data for 10 additional fatty acids. The additional analytes and data are summarized in Table 6.

The data are plotted by analyte in material in the chart section of Appendix A. Each data point is the mean and associated standard deviation of the data reported by the laboratory of the indicated analyte in the indicated sample. The medians calculated from the data received for the fatty acid in each sample are summarized in Table 7 and included for each chart in a text box. Note that after an initial look at the data, Lab 8's data were not included in the median calculations since their data were substantially lower (in many cases an order of magnitude lower) than the consensus data from the other laboratories. The charts for SRM 1950 also include the reference or certified values in the chart title for comparison purposes.

In addition to nomenclature, Table 1 summarizes the number of laboratories reporting data for each fatty acid in each material, the range of mean values reported, and the lab number reporting the highest and lowest values for each fatty acid in each material. Note that lab 8 was included in the summation of the number of laboratories reporting data (including LOD and LOQ) but not in the range of values or identification of lab number reporting the lowest value. Excluding lab 8's data, there are no trends in the data across all fatty acids. There are a few trends with individual fatty acid as can be noted in Table 1. For example, lab 10 reported the lowest mean value for myristic acid and myristoleic acid, and lab 2 reported the highest mean value for cis-vaccenic acid and arachidic acid.

This information is also presented in Table 8 as the percent difference for each laboratory mean ( $n=3)$ from the median data for the particular analyte in the indicated sample. There do appear to be certain trends for individual fatty acids as noted above with data from one or more laboratories being high or low for a particular fatty acid in all of the materials studied. These results could be obtained by incomplete extraction (low), coelution with other fatty acids or matrix components on the analytical column used (high), or from some other method variations such as inaccurate calibration. The methods used by each laboratory are summarized in Appendix A with the procedures in Table A-1 and the 
calibration information in Table A-2. The hydrolysis and extraction methods were generally different among the laboratories with the exception being labs 3 and 4 reporting the use of very similar procedures. All laboratories used gas chromatography (GC) with either flame ionization detection (FID) or mass spectrometry (MS) following a derivatization. For the calibration information (Table A-2), most laboratories used a linear regression with the exception of lab 6 which used a quadratic regression, lab 9 which reported only a weighting factor, lab 10 which did a one point calibration, and lab 11 used a mix of linear and quadratic regression tailored by analyte. Labs 5 and 7 did not report calibration information.

Intercomparison exercises provide an important mechanism for assessing the comparability, repeatability, and trueness of data being produced by the participating laboratories. Exercise materials similar in matrix, form, and analyte concentration to typical samples routinely analyzed by the laboratories are most useful for demonstrating the level of comparability and for revealing potential measurement and method problems. The data from this exercise can be used to assess the comparability across a limited number of international laboratories (see Appendix B). The data do not indicate specific method biases but do indicate the need for such intercomparisons and for the use of control materials to increase the comparability of data across laboratories.

\section{Acknowledgments}

The time and effort of the analysts and management of the participating laboratories are gratefully acknowledged.

\section{Disclaimer}

Certain commercial equipment, instruments, or materials are identified in this report to specify adequately the experimental procedure. Such identification does not imply recommendation or endorsement by the National Institute of Standards and Technology, nor does it imply that the materials or equipment identified are the best available for the purpose. 


\begin{tabular}{|c|c|c|c|c|c|c|c|}
\hline & & mol wt & & \# Labs & range reported & lab reporting & lab reporting \\
\hline & & $\mathrm{g} / \mathrm{mol}$ & material & reporting & $(\mu \mathrm{mol} / \mathrm{L})$ & highest value $^{a}$ & lowest value ${ }^{a}$ \\
\hline \multirow[t]{4}{*}{ C14:0 } & Myristic acid & 228.38 & SRM 2378-1 & 11 & $128-286$ & 7 & 10 \\
\hline & & & SRM 2378-2 & 11 & 86.4-175 & 5 & 10 \\
\hline & & & SRM 2378-3 & 11 & $88.2-224$ & 7 & 10 \\
\hline & & & SRM 1950 & 11 & $61.4-162$ & 7 & 10 \\
\hline \multirow[t]{4}{*}{ C14:1n5 } & Myristoleic acid & 226.38 & SRM 2378-1 & 8 & $7.31-28.5$ & 7 & 10 \\
\hline & & & SRM 2378-2 & 8 & $5.49-19.3$ & 2 & 10 \\
\hline & & & SRM 2378-3 & 8 & $5.69-24.5$ & 7 & 10 \\
\hline & & & SRM 1950 & 8 & $4.31-16.5$ & 7 & 10 \\
\hline \multirow[t]{4}{*}{ C16:0 } & Palmitic acid & 256.43 & SRM 2378-1 & 11 & $1473-3884$ & 7 & 2 \\
\hline & & & SRM 2378-2 & 11 & $1523-3680$ & 5 & 2 \\
\hline & & & SRM 2378-3 & 11 & 1307-3186 & 7 & 2 \\
\hline & & & SRM 1950 & 11 & $1273-3058$ & 7 & 2 \\
\hline \multirow[t]{4}{*}{ C16:1n7 } & Palmitoleic acid & 254.43 & SRM 2378-1 & 11 & $55.1-252$ & 5 & 7 \\
\hline & & & SRM 2378-2 & 11 & $63.9-333$ & 5 & 7 \\
\hline & & & SRM 2378-3 & 11 & $58.6-228$ & 5 & 7 \\
\hline & & & SRM 1950 & 11 & $61.6-338$ & 5 & 7 \\
\hline \multirow[t]{4}{*}{ C18:0 } & Stearic acid & 284.48 & SRM 2378-1 & 11 & $694-1154$ & 7 & 2 \\
\hline & & & SRM 2378-2 & 11 & $696-1122$ & 5 & 10 \\
\hline & & & SRM 2378-3 & 11 & $587-1069$ & 7 & 10 \\
\hline & & & SRM 1950 & 11 & $526-932$ & 7 & 10 \\
\hline \multirow[t]{4}{*}{ C18:1n7 } & cis-Vaccenic acid & 282.48 & SRM 2378-1 & 9 & $118-234$ & 2 & 3 \\
\hline & & & SRM 2378-2 & 9 & $109-264$ & 2 & 3 \\
\hline & & & SRM 2378-3 & 9 & $92.5-212$ & 2 & 3 \\
\hline & & & SRM 1950 & 9 & $129-295$ & 2 & 1 \\
\hline \multirow[t]{4}{*}{ C18:1n9 } & Oleic acid & 282.48 & SRM 2378-1 & 11 & $1113-2788$ & 7 & 2 \\
\hline & & & SRM 2378-2 & 11 & $1303-3285$ & 5 & 2 \\
\hline & & & SRM 2378-3 & 11 & $1077-2603$ & 7 & 2 \\
\hline & & & SRM 1950 & 11 & $1113-2513$ & 7 & 2 \\
\hline \multirow[t]{4}{*}{ C18:2n6 } & Linoleic acid & 280.48 & SRM 2378-1 & 11 & $2631-4228$ & 7 & 10 \\
\hline & & & SRM 2378-2 & 11 & $3180-5328$ & 5 & 7 \\
\hline & & & SRM 2378-3 & 11 & $2331-4024$ & 5 & 10 \\
\hline & & & SRM 1950 & 11 & $2081-3776$ & 7 & 10 \\
\hline \multirow[t]{4}{*}{ C18:3n3 } & alpha-Linolenic acid & 278.48 & SRM 2378-1 & 11 & $79.2-147$ & 7 & 4 \\
\hline & & & SRM 2378-2 & 11 & $78.7-130$ & 5 & 10 \\
\hline & & & SRM 2378-3 & 11 & $40.8-84.6$ & 7 & 4 \\
\hline & & & SRM 1950 & 11 & $36.6-76.2$ & 7 & 10 \\
\hline \multirow[t]{4}{*}{ C18:3n6 } & gamma-Linolenic acid & 278.48 & SRM 2378-1 & 11 & $30.8-57.5$ & 7 & 10 \\
\hline & & & SRM 2378-2 & 11 & $54.4-93.9$ & 5 & 10 \\
\hline & & & SRM 2378-3 & 11 & $34.0-66.2$ & 7 & 10 \\
\hline & & & SRM 1950 & 11 & $36.6-72.9$ & 7 & 10 \\
\hline \multirow[t]{4}{*}{ C20:0 } & Arachidic acid & 312.54 & SRM 2378-1 & 10 & $15.8-68.0$ & 2 & 4 \\
\hline & & & SRM 2378-2 & 10 & $15.2-78.3$ & 2 & 4 \\
\hline & & & SRM 2378-3 & 10 & $12.5-59.0$ & 2 & 4 \\
\hline & & & SRM 1950 & 10 & $11.5-51.7$ & 2 & 4 \\
\hline \multirow[t]{4}{*}{ C20:1n9 } & 11-Eicosenoic acid & 310.54 & SRM 2378-1 & 9 & $11.8-48.1$ & 7 & 4 \\
\hline & & & SRM 2378-2 & 9 & $12.2-37.3$ & 7 & 10 \\
\hline & & & SRM 2378-3 & 9 & $13.1-29.1$ & 7 & 10 \\
\hline & & & SRM 1950 & 9 & $10.3-27.4$ & 7 & 4 \\
\hline
\end{tabular}


Table 1 (cont). Nomenclature used in this study and summary of values reported

\begin{tabular}{|c|c|c|c|c|c|c|c|}
\hline & & $\mathrm{mol} \mathrm{wt}$ & & \# Labs & range reported & reported & reported \\
\hline & & $\mathrm{g} / \mathrm{mol}$ & material & reporting & $(\mu \mathrm{mol} / \mathrm{L})$ & highest value ${ }^{a}$ & lowest value ${ }^{a}$ \\
\hline \multirow[t]{4}{*}{$\mathrm{C} 20: 2 \mathrm{n} 6$} & 11,14-Eicosadienoic acid & 308.53 & SRM 2378-1 & 8 & $8.50-19.8$ & 3 & 7 \\
\hline & & & SRM 2378-2 & 8 & $5.98-25.8$ & 5 & 7 \\
\hline & & & SRM 2378-3 & 8 & $6.59-22.5$ & 5 & 7 \\
\hline & & & SRM 1950 & 8 & $7.78-22.4$ & 5 & 7 \\
\hline \multirow[t]{4}{*}{ C20:3n6 } & homo-gamma-Linolenic acid & 306.53 & SRM 2378-1 & 10 & $59.3-158$ & 7 & 2 \\
\hline & & & SRM 2378-2 & 10 & $89.3-231$ & 5 & 2 \\
\hline & & & SRM 2378-3 & 10 & $62.0-176$ & 7 & 2 \\
\hline & & & SRM 1950 & 10 & $68.0-204$ & 7 & 2 \\
\hline \multirow[t]{4}{*}{ C20:4n6 } & Arachidonic acid & 304.52 & SRM 2378-1 & 11 & $493-987$ & 2 & 4 \\
\hline & & & SRM 2378-2 & 11 & $646-1330$ & 2 & 10 \\
\hline & & & SRM 2378-3 & 11 & $569-1127$ & 2 & 10 \\
\hline & & & SRM 1950 & 11 & $540-1067$ & 2 & 10 \\
\hline \multirow[t]{4}{*}{$C 20: 5 n 3$} & Eicosapentaenoic acid (EPA) & 302.52 & SRM 2378-1 & 11 & $228-370$ & 2 & 10 \\
\hline & & & SRM 2378-2 & 11 & $62.6-115$ & 2 & 10 \\
\hline & & & SRM 2378-3 & 11 & $50.8-87.0$ & 2 and 7 & 10 \\
\hline & & & SRM 1950 & 11 & $35.8-61.7$ & 7 & 10 \\
\hline \multirow[t]{4}{*}{ C22:0 } & Docosanoic acid & 340.59 & SRM 2378-1 & 11 & $5.02-67.1$ & 10 & 7 \\
\hline & & & SRM 2378-2 & 11 & $2.37-92.7$ & 11 & 7 \\
\hline & & & SRM 2378-3 & 11 & $3.53-61.0$ & 11 & 7 \\
\hline & & & SRM 1950 & 11 & $2.95-52.1$ & 11 & 7 \\
\hline \multirow[t]{4}{*}{ C22:1n9 } & Docosenoic acid & 338.59 & SRM 2378-1 & 7 & $3.24-19$ & 9 & 10 \\
\hline & & & SRM 2378-2 & 7 & $2.20-16.3$ & 9 & 10 \\
\hline & & & SRM 2378-3 & 7 & $2.09-19.9$ & 9 & 10 \\
\hline & & & SRM 1950 & 7 & $2.12-20.7$ & 9 & 10 \\
\hline \multirow[t]{4}{*}{$\mathrm{C} 22: 4 \mathrm{n} 6$} & Docosatetraenoic acid & 332.57 & SRM 2378-1 & 10 & $9.00-29.9$ & 9 & 2 \\
\hline & & & SRM 2378-2 & 10 & $17.7-41.8$ & 9 & 2 \\
\hline & & & SRM 2378-3 & 10 & $12.3-32.6$ & 9 & 2 \\
\hline & & & SRM 1950 & 10 & $12.7-41.2$ & 7 & 5 \\
\hline \multirow[t]{4}{*}{$C 22: 5 n 3$} & Docosapentaenoic acid & 330.57 & SRM 2378-1 & 11 & $52.0-99.8$ & 7 & 2 and 4 \\
\hline & & & SRM 2378-2 & 11 & $39.0-55.9$ & 5 & 4 \\
\hline & & & SRM 2378-3 & 11 & $27.0-50.6$ & 7 & 4 \\
\hline & & & SRM 1950 & 11 & $32.7-59.5$ & 7 & 2 \\
\hline \multirow[t]{4}{*}{$\mathrm{C} 22: 5 \mathrm{n} 6$} & Docosapentaenoic acid & 330.57 & SRM 2378-1 & 9 & $6.00-12.8$ & 7 & 2 \\
\hline & & & SRM 2378-2 & 9 & $9.67-16.7$ & 7 & 2 \\
\hline & & & SRM 2378-3 & 9 & $8.67-21.1$ & 7 & 2 \\
\hline & & & SRM 1950 & 9 & $11.7-27.3$ & 7 & 2 \\
\hline \multirow[t]{4}{*}{$C 22: 6 n 3$} & Docosahexaenoic acid (DHA) & 328.57 & SRM 2378-1 & 11 & $241-450$ & 7 & 4 \\
\hline & & & SRM 2378-2 & 11 & 136-195 & 7 & 10 \\
\hline & & & SRM 2378-3 & 11 & $124-251$ & 7 & 4 \\
\hline & & & SRM 1950 & 11 & $82.3-170$ & 7 & 10 \\
\hline \multirow[t]{4}{*}{ C24:0 } & Lignoceric acid & 368.64 & SRM 2378-1 & 11 & $3.77-70.0$ & 10 & 7 \\
\hline & & & SRM 2378-2 & 11 & $2.61-81.9$ & 10 & 7 \\
\hline & & & SRM 2378-3 & 11 & $4.10-56.5$ & 10 & 7 \\
\hline & & & SRM 1950 & 11 & $2.47-47.3$ & 10 & 7 \\
\hline \multirow[t]{4}{*}{ C24:1n9 } & Nervonic acid & 366.63 & SRM 2378-1 & 10 & $18.2-104$ & 10 & 3 \\
\hline & & & SRM 2378-2 & 10 & $20.4-86.4$ & 11 & 4 \\
\hline & & & SRM 2378-3 & 10 & $13.1-63.8$ & 10 & 4 \\
\hline & & & SRM 1950 & 10 & $16.0-72.1$ & 10 & 3 \\
\hline${ }^{\mathrm{a}}$ Excludir & data submitted by Lab 8 & & & & & & \\
\hline
\end{tabular}




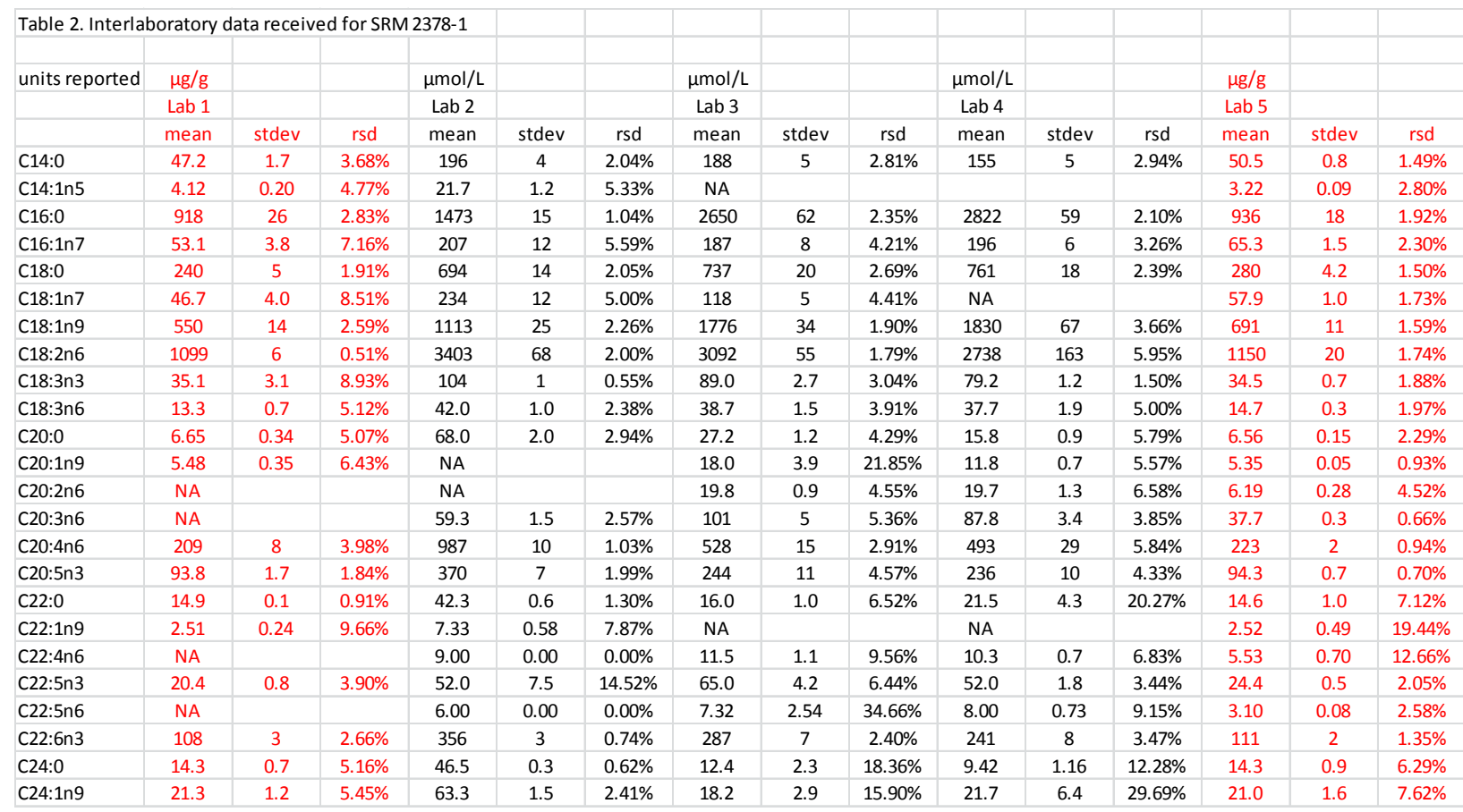

\begin{tabular}{|c|c|c|c|c|c|c|c|c|c|c|c|c|c|c|c|c|c|c|}
\hline \multicolumn{19}{|c|}{ Table 2 (Continued) Interlaboratory data received for SRM 2378-1 } \\
\hline & & & & & & & & & & & & & & & & & & \\
\hline \multirow[t]{3}{*}{ units reported } & $\mu \mathrm{mol} / \mathrm{L}$ & & & $\mu \mathrm{g} / \mathrm{g}$ & & & $\mu \mathrm{mol} / \mathrm{L}$ & & & $\mu g / g$ & & & $\mu \mathrm{mol} / \mathrm{L}$ & & & $\mu \mathrm{mol} / \mathrm{L}$ & & \\
\hline & Lab 6 & & & Lab 7 & & & Lab 8 & & & Lab 9 & & & Lab 10 & & & Lab 11 & & \\
\hline & mean & stdev & rsd & mean & stdev & rsd & mean & stdev & rsd & mean & stdev & rsd & mean & stdev & rsd & mean & stdev & rsd \\
\hline C14:0 & 189 & 4 & $2.20 \%$ & 65.6 & 2.4 & $3.67 \%$ & 17.3 & 0.3 & $1.93 \%$ & 44.8 & 0.493821 & $1.10 \%$ & 128 & 3 & $2.72 \%$ & 198 & 1 & $0.54 \%$ \\
\hline C14:1n5 & NA & & & 6.48 & 0.66 & $10.14 \%$ & $<L O D$ & & & 3.51 & 0.07 & $1.91 \%$ & 7.31 & 0.66 & $8.98 \%$ & 12.3 & 0.3 & $2.58 \%$ \\
\hline C16:0 & 2977 & 74 & $2.48 \%$ & 1000 & 13 & $1.30 \%$ & 387 & 29 & $7.61 \%$ & 774 & 7 & $0.84 \%$ & 2290 & 37 & $1.62 \%$ & 3113 & 20 & $0.64 \%$ \\
\hline C16:1n7 & 210 & 4 & $1.98 \%$ & 14.1 & 1.4 & $9.94 \%$ & 6.04 & 0.93 & $15.39 \%$ & 53.1 & 0.5 & $1.00 \%$ & 150 & 3 & $2.26 \%$ & 207 & 2 & $0.74 \%$ \\
\hline C18:0 & 806 & 4 & $0.45 \%$ & 330 & 8 & $2.30 \%$ & 102 & 5 & $5.25 \%$ & 240 & 2 & $0.71 \%$ & 696 & 15 & $2.18 \%$ & 714 & 9 & $1.26 \%$ \\
\hline C18:1n7 & 143 & 3 & $1.85 \%$ & 42.6 & 11.3 & $26.54 \%$ & 43.3 & 7.4 & $17.12 \%$ & ND & & & 138 & 3 & $2.19 \%$ & 136 & 3 & $1.97 \%$ \\
\hline C18:1n9 & 2017 & 55 & $2.73 \%$ & 791 & 25 & $3.12 \%$ & 71.7 & 5.6 & $7.80 \%$ & 636 & 5 & $0.71 \%$ & 1602 & 23 & $1.42 \%$ & 2076 & 19 & $0.92 \%$ \\
\hline C18:2n6 & 3387 & 112 & $3.32 \%$ & 1191 & 2 & $0.13 \%$ & 130 & 41 & $31.08 \%$ & 955 & 7 & $0.77 \%$ & 2631 & 29 & $1.10 \%$ & 3422 & 32 & $0.94 \%$ \\
\hline C18:3n3 & 104 & 2 & $2.01 \%$ & 41.1 & 0.5 & $1.14 \%$ & $<L O D$ & & & 30.5 & 0.2 & $0.78 \%$ & 80.6 & 0.4 & $0.51 \%$ & 112 & 1 & $0.46 \%$ \\
\hline C18:3n6 & 40.8 & 0.9 & $2.23 \%$ & 16.1 & 0.8 & $4.94 \%$ & 5.18 & 0.96 & $18.45 \%$ & 12.1 & 0.0 & $0.11 \%$ & 30.8 & 0.3 & $1.03 \%$ & 40.3 & 0.2 & $0.48 \%$ \\
\hline C20:0 & NA & & & 8.76 & 0.46 & $5.25 \%$ & $<L O D$ & & & 8.59 & 0.66 & $7.64 \%$ & 25.9 & 0.6 & $2.48 \%$ & 24.9 & 0.5 & $1.89 \%$ \\
\hline C20:1n9 & NA & & & 15.0 & 8.7 & $57.67 \%$ & $<L O D$ & & & 4.96 & 0.27 & $5.36 \%$ & 16.2 & 0.3 & $1.71 \%$ & 16.3 & 0.2 & $1.21 \%$ \\
\hline C20:2n6 & NA & & & 2.63 & 0.58 & $22.14 \%$ & $<L O D$ & & & 6.04 & 0.25 & $4.19 \%$ & 14.0 & 0.6 & $4.04 \%$ & 17.4 & 0.4 & $2.41 \%$ \\
\hline $\mathrm{C} 20: 3 \mathrm{n} 6$ & 104 & 2 & $1.46 \%$ & 48.8 & 0.2 & $0.39 \%$ & 6.53 & 0.96 & $14.68 \%$ & 32.8 & 0.2 & $0.54 \%$ & 94.8 & 2.5 & $2.61 \%$ & 102 & 1 & $1.37 \%$ \\
\hline C20:4n6 & 625 & 13 & $2.06 \%$ & 277 & 3 & $0.99 \%$ & 29.9 & 2.3 & $7.55 \%$ & 194 & 1 & $0.41 \%$ & 533 & 9 & $1.68 \%$ & 634 & 3 & $0.53 \%$ \\
\hline $\mathrm{C} 20: 5 \mathrm{n} 3$ & 257 & 5 & $2.06 \%$ & 112 & 0 & $0.13 \%$ & 9.83 & 1.09 & $11.13 \%$ & 81.4 & 0.6 & $0.71 \%$ & 228 & 2 & $0.96 \%$ & 272 & 7 & $2.41 \%$ \\
\hline C22:0 & 44.5 & 1.1 & $2.47 \%$ & 1.72 & 0.35 & $20.38 \%$ & $<L O D$ & & & 15.7 & 1.3 & $8.08 \%$ & 67.1 & 0.8 & $1.24 \%$ & 58.7 & 0.8 & $1.29 \%$ \\
\hline C22:1n9 & NA & & & & & & $<L O D$ & & & 6.42 & 0.71 & $10.99 \%$ & 3.24 & 0.26 & $7.91 \%$ & 4.21 & 0.62 & $14.70 \%$ \\
\hline $\mathrm{C} 22: 4 \mathrm{n} 6$ & 12.4 & 0.2 & $1.40 \%$ & 7.18 & 0.66 & $9.19 \%$ & $<L O D$ & & & 9.93 & 0.91 & $9.19 \%$ & 13.1 & 0.1 & $0.97 \%$ & 12.4 & 0.11 & $0.85 \%$ \\
\hline C22:5n3 & 66.2 & 1.4 & $2.12 \%$ & 33.1 & 1.0 & $3.14 \%$ & $<\mathrm{LOQ}$ & & & 21.8 & 0.5 & $2.30 \%$ & 65.7 & 2.3 & $3.50 \%$ & 70.5 & 0.5 & $0.64 \%$ \\
\hline C22:5n6 & NA & & & 4.26 & 0.23 & $5.40 \%$ & $<L O D$ & & & 3.01 & 0.09 & $3.12 \%$ & 8.00 & 0.32 & $4.00 \%$ & 8.37 & 0.26 & $3.06 \%$ \\
\hline $\mathrm{C} 22: 6 \mathrm{n} 3$ & 283 & 7 & $2.47 \%$ & 149 & 2 & $1.31 \%$ & 13.0 & 0.8 & $6.34 \%$ & 98.2 & 2.5 & $2.56 \%$ & 276 & 7 & $2.45 \%$ & 320 & 6 & $1.96 \%$ \\
\hline $\mathrm{C} 24: 0$ & 42.2 & 0.5 & $1.09 \%$ & 1.39 & 0.24 & $17.00 \%$ & $<L O D$ & & & 15.7 & 1.5 & $9.78 \%$ & 70.0 & 1.1 & $1.55 \%$ & 56.2 & 0.4 & $0.39 \%$ \\
\hline \multirow{5}{*}{$\mathrm{C} 24: \ln 9$} & 62.5 & 2.7 & $4.28 \%$ & & & & $<L O D$ & & & 21.0 & 1.6 & $7.66 \%$ & 104 & 1 & $1.43 \%$ & 87.1 & 0.8 & $0.80 \%$ \\
\hline & & & & & & & & & & \multicolumn{2}{|c|}{ NOTE for Lab 9: } & & & & & & & \\
\hline & & & & & & & & & & C18:1n9 & Oleic acid & & \multicolumn{4}{|c|}{ Total 18:1 determined \& entered } & & \\
\hline & & & & & & & & & & C20:1n9 & \multicolumn{2}{|c|}{ 11-Eicosenoic acid } & \multicolumn{4}{|c|}{ Total 20:1 determined \& entered } & & \\
\hline & & & & & & & & & & C22:1n9 & \multicolumn{2}{|c|}{ Docosenoic acid } & \multicolumn{4}{|c|}{ Total 22:1 determined \& entered } & & \\
\hline
\end{tabular}




\begin{tabular}{|c|c|c|c|c|c|c|c|c|c|c|c|c|c|c|c|}
\hline \multicolumn{16}{|c|}{ Table 2 (Continued) Interlaboratory data received for SRM 2378-1 } \\
\hline & Lab 1 & & & Lab 2 & & \multirow{3}{*}{ rsd } & Lab 3 & & & Lab 4 & & \multirow[b]{2}{*}{ rsd } & Lab 5 & \multirow{3}{*}{$\begin{array}{l}\text { stdev } \\
0.035\end{array}$} & \multirow{3}{*}{$\begin{array}{c}\text { rsd } \\
3.56 \%\end{array}$} \\
\hline & mean & stdev & rsd & mean & stdev & & mean & stdev & rsd & mean & stdev & & mean & & \\
\hline density $(\mathrm{g} / \mathrm{mL})$ & 1.02 & 0.00 & $0.01 \%$ & \multicolumn{2}{|c|}{ not reported } & & 0.989 & 0.030 & $3.06 \%$ & \multicolumn{2}{|c|}{ not reported } & & 0.983 & & \\
\hline & & & & & & & & & & & & & & & \\
\hline \multicolumn{16}{|c|}{ CONVERT ALL TO $\mu \mathrm{mol} / \mathrm{L}$} \\
\hline & Lab 1 & & & Lab 2 & & & Lab 3 & & & Lab 4 & & & Lab 5 & & \\
\hline & mean & stdev & rsd & mean & stdev & rsd & mean & stdev & rsd & mean & stdev & rsd & mean & stdev & rsd \\
\hline C14:0 & 211 & 8 & $3.68 \%$ & 196 & 4 & $2.04 \%$ & 188 & 5 & $2.81 \%$ & 155 & 5 & $2.94 \%$ & 217 & 3 & $1.49 \%$ \\
\hline C14:1n5 & 18.6 & 0.9 & $4.77 \%$ & 21.7 & 1.2 & $5.33 \%$ & NA & & & & & & 14.0 & 0.4 & $2.80 \%$ \\
\hline C16:0 & 3661 & 103 & $2.83 \%$ & 1473 & 15 & $1.04 \%$ & 2650 & 62 & $2.35 \%$ & 2822 & 59 & $2.10 \%$ & 3588 & 69 & $1.92 \%$ \\
\hline C16:1n7 & 213 & 15 & $7.16 \%$ & 207 & 12 & $5.59 \%$ & 187 & 8 & $4.21 \%$ & 196 & 6 & $3.26 \%$ & 252 & 6 & $2.30 \%$ \\
\hline C18:0 & 863 & 16 & $1.91 \%$ & 694 & 14 & $2.05 \%$ & 737 & 20 & $2.69 \%$ & 761 & 18 & $2.39 \%$ & 968 & 15 & $1.50 \%$ \\
\hline C18:1n7 & 169 & 14 & $8.51 \%$ & 234 & 12 & $5.00 \%$ & 118 & 5 & $4.41 \%$ & NA & & & 201 & 3 & $1.73 \%$ \\
\hline C18:1n9 & 1990 & 51 & $2.59 \%$ & 1113 & 25 & $2.26 \%$ & 1776 & 34 & $1.90 \%$ & 1830 & 67 & $3.66 \%$ & 2405 & 38 & $1.59 \%$ \\
\hline C18:2n6 & 4007 & 20 & $0.51 \%$ & 3403 & 68 & $2.00 \%$ & 3092 & 55 & $1.79 \%$ & 2738 & 163 & $5.95 \%$ & 4030 & 70 & $1.74 \%$ \\
\hline $\mathrm{C} 18: 3 \mathrm{n} 3$ & 129 & 12 & $8.93 \%$ & 104 & 1 & $0.55 \%$ & 89.0 & 2.7 & $3.04 \%$ & 79.2 & 1.2 & $1.50 \%$ & 122 & 2 & $1.88 \%$ \\
\hline C18:3n6 & 48.9 & 2.5 & $5.12 \%$ & 42.0 & 1.0 & $2.38 \%$ & 38.7 & 1.5 & $3.91 \%$ & 37.7 & 1.9 & $5.00 \%$ & 51.9 & 1.0 & $1.97 \%$ \\
\hline C20:0 & 21.7 & 1.1 & $5.07 \%$ & 68.0 & 2.0 & $2.94 \%$ & 27.2 & 1.2 & $4.29 \%$ & 15.8 & 0.9 & $5.79 \%$ & 20.6 & 0.5 & $2.29 \%$ \\
\hline C20:1n9 & 18.1 & 1.2 & $6.43 \%$ & NA & & & 18.0 & 3.9 & $21.85 \%$ & 11.8 & 0.7 & $5.57 \%$ & 16.9 & 0.2 & $0.93 \%$ \\
\hline $\mathrm{C} 20: 2 \mathrm{n} 6$ & NA & & & NA & & & 19.8 & 0.9 & $4.55 \%$ & 19.7 & 1.3 & $6.58 \%$ & 19.7 & 0.9 & $4.52 \%$ \\
\hline $\mathrm{C} 20: 3 \mathrm{n} 6$ & NA & & & 59.3 & 1.5 & $2.57 \%$ & 101 & 5 & $5.36 \%$ & 87.8 & 3.4 & $3.85 \%$ & 121 & 1 & $0.66 \%$ \\
\hline C20:4n6 & 703 & 28 & $3.98 \%$ & 987 & 10 & $1.03 \%$ & 528 & 15 & $2.91 \%$ & 493 & 29 & $5.84 \%$ & 720 & 7 & $0.94 \%$ \\
\hline$C 20: 5 n 3$ & 317 & 6 & $1.84 \%$ & 370 & 7 & $1.99 \%$ & 244 & 11 & $4.57 \%$ & 236 & 10 & $4.33 \%$ & 306 & 2 & $0.70 \%$ \\
\hline C22:0 & 44.8 & 0.4 & $0.91 \%$ & 42.3 & 0.6 & $1.30 \%$ & 16.0 & 1.0 & $6.52 \%$ & 21.5 & 4.3 & $20.27 \%$ & 42.1 & 3.0 & $7.12 \%$ \\
\hline C22:1n9 & 7.57 & 0.73 & $9.66 \%$ & 7.33 & 0.58 & $7.87 \%$ & NA & & & NA & & & 7.32 & 1.42 & $19.44 \%$ \\
\hline $\mathrm{C} 22: 4 \mathrm{n} 6$ & NA & & & 9.00 & 0.00 & $0.00 \%$ & 11.5 & 1.1 & $9.56 \%$ & 10.3 & 0.7 & $6.83 \%$ & 16.3 & 2.1 & $12.66 \%$ \\
\hline $\mathrm{C} 22: 5 \mathrm{n} 3$ & 63.2 & 2.5 & $3.90 \%$ & 52.0 & 7.5 & $14.52 \%$ & 65.0 & 4.2 & $6.44 \%$ & 52.0 & 1.8 & $3.44 \%$ & 72.6 & 1.5 & $2.05 \%$ \\
\hline $\mathrm{C} 22: 5 \mathrm{n} 6$ & NA & & & 6.00 & 0.00 & $0.00 \%$ & 7.32 & 2.54 & $34.66 \%$ & 8.00 & 0.73 & $9.15 \%$ & 9.22 & 0.24 & $2.58 \%$ \\
\hline C22:6n3 & 337 & 9 & $2.66 \%$ & 356 & 3 & $0.74 \%$ & 287 & 7 & $2.40 \%$ & 241 & 8 & $3.47 \%$ & 332 & 4 & $1.35 \%$ \\
\hline C24:0 & 39.6 & 2.0 & $5.16 \%$ & 46.5 & 0.3 & $0.62 \%$ & 12.4 & 2.3 & $18.36 \%$ & 9.42 & 1.16 & $12.28 \%$ & 38.1 & 2.4 & $6.29 \%$ \\
\hline C24:1n9 & 59.3 & 3.2 & $5.45 \%$ & 63.3 & 1.5 & $2.41 \%$ & 18.2 & 2.9 & $15.90 \%$ & 21.7 & 6.4 & $29.69 \%$ & 56.3 & 4.3 & $7.62 \%$ \\
\hline
\end{tabular}

\begin{tabular}{|c|c|c|c|c|c|c|c|c|c|c|c|c|c|c|c|c|c|c|}
\hline & & & \multicolumn{16}{|c|}{ Table 2 (Continued) Interlaboratory data received for SRM 2378-1 } \\
\hline & Lab 6 & & & Lab 7 & & & Lab 8 & & & Lab 9 & & & Lab 10 & & & Lab 11 & & \\
\hline & mean & stdev & rsd & mean & stdev & rsd & mean & stdev & rsd & mean & stdev & rsd & mean & stdev & rsd & mean & stdev & rsd \\
\hline $\operatorname{density~}(\mathrm{g} / \mathrm{mL})$ & 1.04 & 0.00 & $0.41 \%$ & 0.996 & 0.010 & $0.95 \%$ & 1.00 & 0.00 & $0.01 \%$ & \multicolumn{2}{|c|}{ not reported use 1} & & & & & 0.967 & 0.005 & $0.52 \%$ \\
\hline \multirow{2}{*}{\multicolumn{19}{|c|}{ CONVERT ALL TO $\mu \mathrm{mol} / \mathrm{L}$}} \\
\hline & & & & & & & & & & & & & & & & & & \\
\hline & Lab 6 & & & Lab 7 & & & Lab 8 & & & Lab 9 & & & Lab 10 & & & Lab 11 & & \\
\hline & mean & stdev & rsd & mean & stdev & rsd & mean & stdev & rsd & mean & stdev & rsd & mean & stdev & rsd & mean & stdev & rsd \\
\hline C14:0 & 189 & 4 & $2.20 \%$ & 286 & 11 & $3.67 \%$ & 17.3 & 0.3 & $1.93 \%$ & 196 & 2 & $1.10 \%$ & 128 & 3 & $2.72 \%$ & 198 & 1 & $0.54 \%$ \\
\hline C14:1n5 & NA & & & 28.5 & 2.9 & $10.14 \%$ & $<L O D$ & & & 15.5 & 0.3 & $1.91 \%$ & 7.31 & 0.66 & $8.98 \%$ & 12.3 & 0.3 & $2.58 \%$ \\
\hline C16:0 & 2977 & 74 & $2.48 \%$ & 3884 & 50 & $1.30 \%$ & 387 & 29 & $7.61 \%$ & 3019 & 25 & $0.84 \%$ & 2290 & 37 & $1.62 \%$ & 3113 & 20 & $0.64 \%$ \\
\hline C16:1n7 & 210 & 4 & $1.98 \%$ & 55.1 & 5.5 & $9.94 \%$ & 6.04 & 0.93 & $15.39 \%$ & 209 & 2 & $1.00 \%$ & 150 & 3 & $2.26 \%$ & 207 & 2 & $0.74 \%$ \\
\hline C18:0 & 806 & 4 & $0.45 \%$ & 1154 & 27 & $2.30 \%$ & 102 & 5 & $5.25 \%$ & 842 & 6 & $0.71 \%$ & 696 & 15 & $2.18 \%$ & 714 & 9 & $1.26 \%$ \\
\hline C18:1n7 & 143 & 3 & $1.85 \%$ & 150 & 40 & $26.54 \%$ & 43.3 & 7.4 & $17.12 \%$ & NA & & & 138 & 3 & $2.19 \%$ & 136 & 3 & $1.97 \%$ \\
\hline C18:1n9 & 2017 & 55 & $2.73 \%$ & 2788 & 87 & $3.12 \%$ & 71.7 & 5.6 & $7.80 \%$ & 2250 & 16 & $0.71 \%$ & 1602 & 23 & $1.42 \%$ & 2076 & 19 & $0.92 \%$ \\
\hline C18:2n6 & 3387 & 112 & $3.32 \%$ & 4228 & 6 & $0.13 \%$ & 130 & 41 & $31.08 \%$ & 3403 & 26 & $0.77 \%$ & 2631 & 29 & $1.10 \%$ & 3422 & 32 & $0.94 \%$ \\
\hline C18:3n3 & 104 & 2 & $2.01 \%$ & 147 & 2 & $1.14 \%$ & $<L O D$ & & & 110 & 1 & $0.78 \%$ & 80.6 & 0.4 & $0.51 \%$ & 112 & 1 & $0.46 \%$ \\
\hline C18:3n6 & 40.8 & 0.9 & $2.23 \%$ & 57.5 & 2.8 & $4.94 \%$ & 5.18 & 0.96 & $18.45 \%$ & 43.5 & 0.0 & $0.11 \%$ & 30.8 & 0.3 & $1.03 \%$ & 40.3 & 0.2 & $0.48 \%$ \\
\hline C20:0 & NA & & & 27.9 & 1.5 & $5.25 \%$ & $<L O D$ & & & 27.5 & 2.1 & $7.64 \%$ & 25.9 & 0.6 & $2.48 \%$ & 24.9 & 0.5 & $1.89 \%$ \\
\hline C20:1n9 & NA & & & 48.1 & 27.7 & $57.67 \%$ & $<L O D$ & & & 16.0 & 0.9 & $5.36 \%$ & 16.2 & 0.3 & $1.71 \%$ & 16.3 & 0.2 & $1.21 \%$ \\
\hline $\mathrm{C} 20: 2 \mathrm{n} 6$ & NA & & & 8.50 & 1.88 & $22.14 \%$ & $<L O D$ & & & 19.6 & 0.8 & $4.19 \%$ & 14.0 & 0.6 & $4.04 \%$ & 17.4 & 0.4 & $2.41 \%$ \\
\hline $\mathrm{C} 20: 3 \mathrm{n} 6$ & 104 & 2 & $1.46 \%$ & 158 & 1 & $0.39 \%$ & 6.53 & 0.96 & $14.68 \%$ & 107 & 1 & $0.54 \%$ & 94.8 & 2.5 & $2.61 \%$ & 102 & 1 & $1.37 \%$ \\
\hline $\mathrm{C} 20: 4 \mathrm{n} 6$ & 625 & 13 & $2.06 \%$ & 908 & 9 & $0.99 \%$ & 29.9 & 2.3 & $7.55 \%$ & 637 & 3 & $0.41 \%$ & 533 & 9 & $1.68 \%$ & 634 & 3 & $0.53 \%$ \\
\hline $\mathrm{C} 20: 5 \mathrm{n} 3$ & 257 & 5 & $2.06 \%$ & 368 & 0 & $0.13 \%$ & 9.83 & 1.09 & $11.13 \%$ & 269 & 2 & $0.71 \%$ & 228 & 2 & $0.96 \%$ & 272 & 7 & $2.41 \%$ \\
\hline C22:0 & 44.5 & 1.1 & $2.47 \%$ & 5.02 & 1.02 & $20.38 \%$ & $<L O D$ & & & 46.2 & 3.7 & $8.08 \%$ & 67.1 & 0.8 & $1.24 \%$ & 58.7 & 0.8 & $1.29 \%$ \\
\hline C22:1n9 & NA & & & & & & $<L O D$ & & & 19.0 & 2.1 & $10.99 \%$ & 3.24 & 0.26 & $7.91 \%$ & 4.21 & 0.62 & $14.70 \%$ \\
\hline $\mathrm{C} 22: 4 \mathrm{n} 6$ & 12.4 & 0.2 & $1.40 \%$ & 21.5 & 2.0 & $9.19 \%$ & $<L O D$ & & & 29.9 & 2.7 & $9.19 \%$ & 13.1 & 0.1 & $0.97 \%$ & 12.4 & 0.11 & $0.85 \%$ \\
\hline $\mathrm{C} 22: 5 \mathrm{n} 3$ & 66.2 & 1.4 & $2.12 \%$ & 99.8 & 3.1 & $3.14 \%$ & $<\mathrm{LOQ}$ & & & 66.1 & 1.5 & $2.30 \%$ & 65.7 & 2.3 & $3.50 \%$ & 70.5 & 0.5 & $0.64 \%$ \\
\hline $\mathrm{C} 22: 5 \mathrm{n} 6$ & NA & & & 12.8 & 0.7 & $5.40 \%$ & $<L O D$ & & & 9.12 & 0.28 & $3.12 \%$ & 8.00 & 0.32 & $4.00 \%$ & 8.37 & 0.26 & $3.06 \%$ \\
\hline $\mathrm{C} 22: 6 \mathrm{n} 3$ & 283 & 7 & $2.47 \%$ & 450 & 6 & $1.31 \%$ & 13.0 & 0.8 & $6.34 \%$ & 299 & 8 & $2.56 \%$ & 276 & 7 & $2.45 \%$ & 320 & 6 & $1.96 \%$ \\
\hline C24:0 & 42.2 & 0.5 & $1.09 \%$ & 3.77 & 0.64 & $17.00 \%$ & $<L O D$ & & & 42.5 & 4.2 & $9.78 \%$ & 70.0 & 1.1 & $1.55 \%$ & 56.2 & 0.4 & $0.39 \%$ \\
\hline \multirow[t]{5}{*}{ C24:1n9 } & 62.5 & 2.7 & $4.28 \%$ & & & & $<L O D$ & & & 57.3 & 4.4 & $7.66 \%$ & 104 & 1 & $1.43 \%$ & 87.1 & 0.8 & $0.80 \%$ \\
\hline & & & & & & & & & & \multicolumn{2}{|c|}{ NOTE for Lab 9: } & & & & & & & \\
\hline & & & & & & & & & & C18:1n9 & Oleic acid & & \multicolumn{4}{|c|}{ Total 18:1 determined \& entered } & & \\
\hline & & & & & & & & & & C20:1n9 & \multicolumn{2}{|c|}{ 11-Eicosenoic acid } & \multicolumn{4}{|c|}{ Total 20:1 determined \& entered } & & \\
\hline & & & & & & & & & & C22:1n9 & \multicolumn{2}{|c|}{ Docosenoic acid } & \multicolumn{4}{|c|}{ Total 22:1 determined \& entered } & & \\
\hline
\end{tabular}




\begin{tabular}{|c|c|c|c|c|c|c|c|c|c|c|c|c|c|c|c|}
\hline Table 3. Interlat & oratory & a receiv & d for SRN & $2378-2$ & & & & & & & & & & & \\
\hline \multirow[t]{3}{*}{ units reported } & $\mu \mathrm{g} / \mathrm{g}$ & & & $\mu \mathrm{mol} / \mathrm{L}$ & & & $\mu \mathrm{mol} / \mathrm{L}$ & & & $\mu \mathrm{mol} / \mathrm{L}$ & & & $\mu \mathrm{g} / \mathrm{g}$ & & \\
\hline & Lab 1 & & & Lab 2 & & & Lab 3 & & & Lab 4 & & & Lab 5 & & \\
\hline & mean & stdev & rsd & mean & stdev & rsd & mean & stdev & rsd & mean & stdev & rsd & mean & stdev & rsd \\
\hline C14:0 & 35.4 & 2.4 & $6.82 \%$ & 158 & 1 & $0.45 \%$ & 153 & 8 & $5.41 \%$ & 126 & 8 & $6.13 \%$ & 39.6 & 2.0 & $5.16 \%$ \\
\hline C14:1n5 & 3.38 & 0.28 & $8.18 \%$ & 19.3 & 0.6 & $2.99 \%$ & NA & & & & & & 2.55 & 0.12 & $4.71 \%$ \\
\hline C16:0 & 719 & 22 & $3.13 \%$ & 1523 & 32 & $2.11 \%$ & 2811 & 122 & $4.35 \%$ & 2762 & 142 & $5.15 \%$ & 935 & 26 & $2.78 \%$ \\
\hline C16:1n7 & 71.5 & 1.9 & $2.72 \%$ & 264 & 13 & $4.74 \%$ & 262 & 16 & $5.99 \%$ & 242 & 11 & $4.57 \%$ & 84.0 & 2.1 & $2.46 \%$ \\
\hline C18:0 & 246 & 7 & $2.88 \%$ & 785 & 22 & $2.80 \%$ & 864 & 29 & $3.41 \%$ & 848 & 55 & $6.49 \%$ & 316 & 10 & $3.01 \%$ \\
\hline C18:1n7 & 31.5 & 1.4 & $4.48 \%$ & 264 & 4 & $1.33 \%$ & 109 & 7 & $6.46 \%$ & NA & & & 62.0 & 0.8 & $1.32 \%$ \\
\hline C18:1n9 & 707 & 10 & $1.36 \%$ & 1303 & 64 & $4.93 \%$ & 2611 & 152 & $5.81 \%$ & 2461 & 214 & $8.68 \%$ & 919 & 13 & $1.41 \%$ \\
\hline C18:2n6 & 1194 & 51 & $4.24 \%$ & 4067 & 64 & $1.58 \%$ & 4378 & 255 & $5.83 \%$ & 3615 & 415 & $11.48 \%$ & 1480 & 20 & $1.35 \%$ \\
\hline C18:3n3 & 31.0 & 1.2 & $3.77 \%$ & 116 & 5 & $3.88 \%$ & 96.6 & 6.8 & $7.04 \%$ & 86.6 & 9.5 & $10.99 \%$ & 36.0 & 0.5 & $1.25 \%$ \\
\hline C18:3n6 & 20.4 & 1.0 & $4.82 \%$ & 82.0 & 4.6 & $5.59 \%$ & 71.3 & 7.7 & $10.81 \%$ & 65.0 & 5.6 & $8.58 \%$ & 25.9 & 0.3 & $1.24 \%$ \\
\hline $\mathrm{C} 20: 0$ & 7.05 & 0.37 & $5.20 \%$ & 78.3 & 2.3 & $2.95 \%$ & 28.7 & 3.8 & $13.15 \%$ & 15.2 & 3.5 & $23.05 \%$ & 7.39 & 0.66 & $8.93 \%$ \\
\hline $\mathrm{C} 20: 1 \mathrm{n} 9$ & 4.92 & 0.23 & $4.63 \%$ & NA & & & 20.7 & 2.4 & $11.59 \%$ & 12.3 & 1.2 & $9.45 \%$ & 5.24 & 0.20 & $3.72 \%$ \\
\hline $\mathrm{C} 20: 2 \mathrm{n} 6$ & NA & & & NA & & & 24.5 & 1.8 & $7.40 \%$ & 17.4 & 0.8 & $4.82 \%$ & 7.90 & 0.15 & $1.90 \%$ \\
\hline C20:3n6 & NA & & & 89.3 & 1.2 & $1.29 \%$ & 200 & 11 & $5.42 \%$ & 167 & 14 & $8.36 \%$ & 70.1 & 0.6 & $0.84 \%$ \\
\hline $\mathrm{C} 20: 4 \mathrm{n} 6$ & 225 & 8 & $3.61 \%$ & 1330 & 56 & $4.19 \%$ & 746 & 36 & $4.88 \%$ & 668 & 72 & $10.74 \%$ & 293 & 3 & $0.86 \%$ \\
\hline $\mathrm{C} 20: 5 \mathrm{n} 3$ & 21.9 & 0.6 & $2.67 \%$ & 115 & 3 & $2.79 \%$ & 64.4 & 9.1 & $14.10 \%$ & 76.2 & 6.3 & $8.28 \%$ & 24.7 & 0.2 & $0.81 \%$ \\
\hline C22:0 & 21.1 & 1.3 & $5.99 \%$ & 59.2 & 0.3 & $0.54 \%$ & 27.8 & 6.2 & $22.35 \%$ & 33.9 & 5.2 & $15.32 \%$ & 21.2 & 2.0 & $9.42 \%$ \\
\hline C22:1n9 & 2.50 & 0.06 & $2.54 \%$ & 7.00 & 0.00 & $0.00 \%$ & NA & & & NA & & & 3.15 & 0.12 & $3.81 \%$ \\
\hline $\mathrm{C} 22: 4 \mathrm{n} 6$ & NA & & & 17.7 & 0.6 & $3.27 \%$ & 21.0 & 3.2 & $15.04 \%$ & 17.8 & 2.3 & $12.94 \%$ & 7.66 & 1.03 & $13.45 \%$ \\
\hline $\mathrm{C} 22: 5 \mathrm{n} 3$ & 17.8 & 0.2 & $0.87 \%$ & 45.3 & 4.0 & $8.91 \%$ & 49.3 & 3.8 & $7.81 \%$ & 39.0 & 1.7 & $4.30 \%$ & 18.3 & 0.5 & $2.73 \%$ \\
\hline $\mathrm{C} 22: 5 \mathrm{n} 6$ & NA & & & 9.67 & 1.53 & $15.80 \%$ & 12.0 & 1.5 & $12.18 \%$ & 13.4 & 0.7 & $4.84 \%$ & 5.13 & 0.20 & $3.90 \%$ \\
\hline $\mathrm{C} 22: 6 \mathrm{n} 3$ & 58.0 & 3.0 & $5.19 \%$ & 184 & 13 & $7.11 \%$ & 163 & 10 & $6.28 \%$ & 145 & 2 & $1.43 \%$ & 59.3 & 0.4 & $0.61 \%$ \\
\hline C24:0 & 16.1 & 1.9 & $11.57 \%$ & 60.2 & 1.5 & $2.45 \%$ & 18.4 & 7.2 & $39.06 \%$ & 21.2 & 0.9 & $4.42 \%$ & 17.1 & 2.2 & $13.01 \%$ \\
\hline C24:1n9 & 17.6 & 1.0 & $5.83 \%$ & 67.3 & 2.1 & $3.09 \%$ & 21.9 & 9.0 & $41.35 \%$ & 20.4 & 6.0 & $29.67 \%$ & 18.8 & 1.9 & $9.88 \%$ \\
\hline
\end{tabular}

\begin{tabular}{|c|c|c|c|c|c|c|c|c|c|c|c|c|c|c|c|c|c|c|}
\hline \multicolumn{19}{|c|}{ Table 3 (continued) Interlaboratory data received for SRM 2378-2 } \\
\hline & & & & & & & & & & & & & & & & & & \\
\hline \multirow[t]{3}{*}{ units reported } & $\mu \mathrm{mol} / \mathrm{L}$ & & & $\mu \mathrm{g} / \mathrm{g}$ & & & $\mu \mathrm{mol} / \mathrm{L}$ & & & $\mu \mathrm{g} / \mathrm{g}$ & & & $\mu \mathrm{mol} / \mathrm{L}$ & & & $\mu \mathrm{mol} / \mathrm{L}$ & & \\
\hline & Lab 6 & & & Lab 7 & & & Lab 8 & & & Lab 9 & & & Lab 10 & & & Lab 11 & & \\
\hline & mean & stdev & rsd & mean & stdev & rsd & mean & stdev & rsd & mean & stdev & rsd & mean & stdev & rsd & mean & stdev & rsd \\
\hline C14:0 & 142 & 3 & $2.11 \%$ & 35.0 & 3.4 & $9.60 \%$ & 19.9 & 1.0 & $4.80 \%$ & 34.3 & 1.1 & $3.20 \%$ & 86.4 & 0.8 & $0.88 \%$ & 152 & 1 & $0.63 \%$ \\
\hline C14:1n5 & NA & & & 3.42 & 0.31 & $8.95 \%$ & $<L O D$ & & & 2.75 & 0.19 & $6.86 \%$ & 5.49 & 0.62 & $11.35 \%$ & 9.24 & 0.16 & $1.68 \%$ \\
\hline C16:0 & 2963 & 87 & $2.95 \%$ & 717 & 85 & $11.81 \%$ & 299 & 16 & $5.24 \%$ & 768 & 31 & $4.07 \%$ & 2057 & 15 & $0.72 \%$ & 3151 & 20 & $0.62 \%$ \\
\hline C16:1n7 & 266 & 7 & $2.56 \%$ & 16.3 & 1.7 & $10.47 \%$ & 8.08 & 0.81 & $10.07 \%$ & 65.7 & 2.8 & $4.20 \%$ & 182 & 3 & $1.80 \%$ & 264 & 4 & $1.48 \%$ \\
\hline C18:0 & 905 & 12 & $1.33 \%$ & 285 & 27 & $9.47 \%$ & 102 & 10 & $9.86 \%$ & 264 & 12 & $4.69 \%$ & 696 & 4 & $0.64 \%$ & 808 & 3 & $0.38 \%$ \\
\hline C18:1n7 & 146 & 0 & $0.00 \%$ & 33.5 & 3.1 & $9.13 \%$ & 36.2 & 2.4 & $6.55 \%$ & ND & & & 130 & 8 & $6.06 \%$ & 140 & 1 & $0.90 \%$ \\
\hline C18:1n9 & 2680 & 85 & $3.19 \%$ & 702 & 87 & $12.45 \%$ & 127 & 7 & $5.79 \%$ & 827 & 29 & $3.51 \%$ & 1984 & 23 & $1.17 \%$ & 2764 & 23 & $0.84 \%$ \\
\hline C18:2n6 & 4353 & 172 & $3.96 \%$ & 896 & 100 & $11.12 \%$ & 100 & 6 & $6.44 \%$ & 1221 & 41 & $3.38 \%$ & 3200 & 40 & $1.24 \%$ & 4479 & 37 & $0.81 \%$ \\
\hline C18:3n3 & 108 & 2 & $1.85 \%$ & 29.9 & 4.7 & $15.80 \%$ & $<L O D$ & & & 31.2 & 1.7 & $5.36 \%$ & 78.7 & 0.7 & $0.85 \%$ & 118 & 1 & $1.25 \%$ \\
\hline C18:3n6 & 74.3 & 1.2 & $1.62 \%$ & 15.3 & 2.3 & $14.92 \%$ & 5.47 & 0.29 & $5.26 \%$ & 21.2 & 0.7 & $3.54 \%$ & 54.4 & 1.1 & $2.07 \%$ & 76.3 & 0.7 & $0.87 \%$ \\
\hline C2O:0 & NA & & & 7.84 & 1.12 & $14.25 \%$ & $<L O D$ & & & 10.6 & 1.6 & $15.29 \%$ & 26.1 & 0.4 & $1.64 \%$ & 29.4 & 0.3 & $0.84 \%$ \\
\hline C20:1n9 & NA & & & 11.6 & 3.82 & $32.85 \%$ & $<L O D$ & & & 4.46 & 0.53 & $11.95 \%$ & 12.2 & 0.4 & $3.13 \%$ & 16.0 & 0.3 & $1.96 \%$ \\
\hline C20:2n6 & NA & & & 1.86 & 0.51 & $27.55 \%$ & $<L O D$ & & & 7.66 & 0.45 & $5.92 \%$ & 19.4 & 0.5 & $2.82 \%$ & 23.1 & 0.2 & $0.87 \%$ \\
\hline C20:3n6 & 189 & 3 & $1.52 \%$ & 64.5 & 7.1 & $11.06 \%$ & 7.94 & 0.50 & $6.32 \%$ & 58.1 & 3.3 & $5.62 \%$ & 156 & 2 & $1.25 \%$ & 196 & 7 & $3.35 \%$ \\
\hline C20:4n6 & 823 & 17 & $2.07 \%$ & 239 & 26 & $10.89 \%$ & 33.9 & 1.8 & $5.20 \%$ & 247 & 15 & $6.03 \%$ & 646 & 9 & $1.42 \%$ & 856 & 8 & $0.94 \%$ \\
\hline $\mathrm{C} 20: 5 \mathrm{n} 3$ & 68.5 & 1.1 & $1.55 \%$ & 20.7 & 1.9 & $8.95 \%$ & $<L O D$ & & & 20.4 & 1.5 & $7.59 \%$ & 62.6 & 0.2 & $0.30 \%$ & 71.9 & 0.5 & $0.73 \%$ \\
\hline C22:0 & 69.7 & 1.9 & $2.66 \%$ & 0.810 & 0.072 & $8.89 \%$ & $<L O D$ & & & 22.7 & 3.8 & $16.55 \%$ & 88.6 & 1.6 & $1.85 \%$ & 92.7 & 1.2 & $1.30 \%$ \\
\hline C22:1n9 & NA & & & & & & $<L O D$ & & & 5.52 & 1.11 & $20.05 \%$ & 2.20 & 0.31 & $14.02 \%$ & 4.46 & 0.32 & $7.19 \%$ \\
\hline C22:4n6 & 21.3 & 0.8 & $3.79 \%$ & 9.14 & 0.85 & $9.24 \%$ & $\angle L O D$ & & & 13.9 & 0.7 & $5.23 \%$ & 20.2 & 0.5 & $2.55 \%$ & 23.0 & 1.3 & $5.81 \%$ \\
\hline $\mathrm{C} 22: 5 \mathrm{n} 3$ & 48.3 & 1.1 & $2.28 \%$ & 17.8 & 2.7 & $15.16 \%$ & $<L O D$ & & & 15.3 & 0.9 & $6.03 \%$ & 44.3 & 0.3 & $0.69 \%$ & 52.1 & 1.2 & $2.35 \%$ \\
\hline C22:5n6 & NA & & & 5.54 & 1.17 & $21.13 \%$ & $<L O D$ & & & 4.39 & 1.17 & $26.73 \%$ & 14.5 & 0.3 & $2.35 \%$ & 16.1 & 0.5 & $3.14 \%$ \\
\hline C22:6n3 & 156 & 3 & $1.85 \%$ & 64.3 & 5.4 & $8.45 \%$ & 4.53 & 0.61 & $13.44 \%$ & 52.5 & 3.3 & $6.27 \%$ & 136 & 2 & $1.31 \%$ & 179 & 1 & $0.33 \%$ \\
\hline C24:0 & 58.3 & 2.5 & $4.23 \%$ & 0.966 & 0.065 & $6.73 \%$ & $<L O D$ & & & 20.0 & 3.6 & $17.87 \%$ & 81.9 & 2.7 & $3.29 \%$ & 78.0 & 1.0 & $1.33 \%$ \\
\hline \multirow[t]{5}{*}{ C24:1n9 } & 61.6 & 3.4 & $5.44 \%$ & & & & $<L O D$ & & & 19.4 & 3.7 & $19.12 \%$ & 84.4 & 1.7 & $1.99 \%$ & 86.4 & 2.6 & $2.95 \%$ \\
\hline & & & & & & & & & & \multicolumn{2}{|c|}{ NOTE for Lab 9: } & & & & & & & \\
\hline & & & & & & & & & & C18:1n9 & Oleic acid & & \multicolumn{4}{|c|}{ Total 18:1 determined \& entered } & & \\
\hline & & & & & & & & & & C20:1n9 & \multicolumn{2}{|c|}{ 11-Eicosenoic acid } & \multicolumn{4}{|c|}{ Total 20:1 determined \& entered } & & \\
\hline & & & & & & & & & & C22:1n9 & \multicolumn{2}{|c|}{ Docosenoic acid } & \multicolumn{4}{|c|}{ Total 22:1 determined \& entered } & & \\
\hline
\end{tabular}




\begin{tabular}{|c|c|c|c|c|c|c|c|c|c|c|c|c|c|c|c|}
\hline \multicolumn{16}{|c|}{ Table 3 (continued) Interlaboratory data received for SRM 2378-2 } \\
\hline & Lab 1 & & & Lab 2 & & \multirow{3}{*}{ rsd } & Lab 3 & & & Lab 4 & & \multirow{3}{*}{ rsd } & Lab 5 & & \multirow{3}{*}{$\begin{array}{c}\text { rsd } \\
2.03 \%\end{array}$} \\
\hline & mean & stdev & rsd & mean & stdev & & mean & stdev & rsd & mean & stdev & & mean & stdev & \\
\hline density $(\mathrm{g} / \mathrm{mL})$ & 1.02 & 0.00 & $0.01 \%$ & \multicolumn{2}{|c|}{ not reported } & & 1.06 & 0.04 & $3.45 \%$ & \multicolumn{2}{|c|}{ not reported } & & 1.01 & 0.02 & \\
\hline \multicolumn{16}{|c|}{ CONVERT ALL TO $\mu \mathrm{mol} / \mathrm{L}$} \\
\hline & Lab 1 & & & Lab 2 & & & Lab 3 & & & Lab 4 & & & Lab 5 & & \\
\hline & mean & stdev & rsd & mean & stdev & rsd & mean & stdev & rsd & mean & stdev & rsd & mean & stdev & rsd \\
\hline C14:0 & 158 & 11 & $6.82 \%$ & 158 & 1 & $0.45 \%$ & 153 & 8 & $5.41 \%$ & 126 & 8 & $6.13 \%$ & 175 & 9 & $5.16 \%$ \\
\hline C14:1n5 & 15.3 & 1.2 & $8.18 \%$ & 19.3 & 0.6 & $2.99 \%$ & NA & & & & & & 11.4 & 0.5 & $4.71 \%$ \\
\hline C16:0 & 2870 & 90 & $3.13 \%$ & 1523 & 32 & $2.11 \%$ & 2811 & 122 & $4.35 \%$ & 2762 & 142 & $5.15 \%$ & 3680 & 102 & $2.78 \%$ \\
\hline C16:1n7 & 288 & 8 & $2.72 \%$ & 264 & 13 & $4.74 \%$ & 262 & 16 & $5.99 \%$ & 242 & 11 & $4.57 \%$ & 333 & 8 & $2.46 \%$ \\
\hline C18:0 & 886 & 26 & $2.88 \%$ & 785 & 22 & $2.80 \%$ & 864 & 29 & $3.41 \%$ & 848 & 55 & $6.49 \%$ & 1122 & 34 & $3.01 \%$ \\
\hline C18:1n7 & 114 & 5 & $4.48 \%$ & 264 & 4 & $1.33 \%$ & 109 & 7 & $6.46 \%$ & NA & & & 222 & 3 & $1.32 \%$ \\
\hline C18:1n9 & 2561 & 35 & $1.36 \%$ & 1303 & 64 & $4.93 \%$ & 2611 & 152 & $5.81 \%$ & 2461 & 214 & $8.68 \%$ & 3285 & 46 & $1.41 \%$ \\
\hline C18:2n6 & 4355 & 185 & $4.24 \%$ & 4067 & 64 & $1.58 \%$ & 4378 & 255 & $5.83 \%$ & 3615 & 415 & $11.48 \%$ & 5328 & 72 & $1.35 \%$ \\
\hline C18:3n3 & 114 & 4 & $3.77 \%$ & 116 & 5 & $3.88 \%$ & 96.6 & 6.8 & $7.04 \%$ & 86.6 & 9.5 & $10.99 \%$ & 130 & 2 & $1.25 \%$ \\
\hline C18:3n6 & 74.8 & 3.6 & $4.82 \%$ & 82.0 & 4.6 & $5.59 \%$ & 71.3 & 7.7 & $10.81 \%$ & 65.0 & 5.6 & $8.58 \%$ & 93.9 & 1.2 & $1.24 \%$ \\
\hline C2O:0 & 23.1 & 1.2 & $5.20 \%$ & 78.3 & 2.3 & $2.95 \%$ & 28.7 & 3.8 & $13.15 \%$ & 15.2 & 3.5 & $23.05 \%$ & 23.9 & 2.1 & $8.93 \%$ \\
\hline C20:1n9 & 16.2 & 0.8 & $4.63 \%$ & NA & & & 20.7 & 2.4 & $11.59 \%$ & 12.3 & 1.2 & $9.45 \%$ & 17.0 & 0.6 & $3.72 \%$ \\
\hline C20:2n6 & NA & & & NA & & & 24.5 & 1.8 & $7.40 \%$ & 17.4 & 0.8 & $4.82 \%$ & 25.8 & 0.5 & $1.90 \%$ \\
\hline C20:3n6 & NA & & & 89.3 & 1.2 & $1.29 \%$ & 200 & 11 & $5.42 \%$ & 167 & 14 & $8.36 \%$ & 231 & 2 & $0.84 \%$ \\
\hline C20:4n6 & 757 & 27 & $3.61 \%$ & 1330 & 56 & $4.19 \%$ & 746 & 36 & $4.88 \%$ & 668 & 72 & $10.74 \%$ & 972 & 8 & $0.86 \%$ \\
\hline C20:5n3 & 74.0 & 2.0 & $2.67 \%$ & 115 & 3 & $2.79 \%$ & 64.4 & 9.1 & $14.10 \%$ & 76.2 & 6.3 & $8.28 \%$ & 82.4 & 0.7 & $0.81 \%$ \\
\hline C22:0 & 63.5 & 3.8 & $5.99 \%$ & 59.2 & 0.3 & $0.54 \%$ & 27.8 & 6.2 & $22.35 \%$ & 33.9 & 5.2 & $15.32 \%$ & 62.9 & 5.9 & $9.42 \%$ \\
\hline C22:1n9 & 7.54 & 0.19 & $2.54 \%$ & 7.00 & 0.00 & $0.00 \%$ & NA & & & NA & & & 9.39 & 0.36 & $3.81 \%$ \\
\hline $\mathrm{C} 22: 4 \mathrm{n} 6$ & NA & & & 17.7 & 0.6 & $3.27 \%$ & 21.0 & 3.2 & $15.04 \%$ & 17.8 & 2.3 & $12.94 \%$ & 23.3 & 3.1 & $13.45 \%$ \\
\hline$C 22: 5 n 3$ & 55.0 & 0.5 & $0.87 \%$ & 45.3 & 4.0 & $8.91 \%$ & 49.3 & 3.8 & $7.81 \%$ & 39.0 & 1.7 & $4.30 \%$ & 55.9 & 1.5 & $2.73 \%$ \\
\hline C22:5n6 & NA & & & 9.67 & 1.53 & $15.80 \%$ & 12.0 & 1.5 & $12.18 \%$ & 13.4 & 0.7 & $4.84 \%$ & 15.7 & 0.6 & $3.90 \%$ \\
\hline$C 22: 6 n 3$ & 181 & 9 & $5.19 \%$ & 184 & 13 & $7.11 \%$ & 163 & 10 & $6.28 \%$ & 145 & 2 & $1.43 \%$ & 182 & 1 & $0.61 \%$ \\
\hline $\mathrm{C} 24: 0$ & 44.7 & 5.2 & $11.57 \%$ & 60.2 & 1.5 & $2.45 \%$ & 18.4 & 7.2 & $39.06 \%$ & 21.2 & 0.9 & $4.42 \%$ & 46.8 & 6.1 & $13.01 \%$ \\
\hline C24:1n9 & 49.2 & 2.9 & $5.83 \%$ & 67.3 & 2.1 & $3.09 \%$ & 21.9 & 9.0 & $41.35 \%$ & 20.4 & 6.0 & $29.67 \%$ & 51.9 & 5.1 & $9.88 \%$ \\
\hline
\end{tabular}

\begin{tabular}{|c|c|c|c|c|c|c|c|c|c|c|c|c|c|c|c|c|c|c|}
\hline \multicolumn{19}{|c|}{ Table 3 (continued) Interlaboratory data received for SRM 2378-2 } \\
\hline & Lab 6 & & & Lab 7 & & & Lab 8 & & & Lab 9 & & & Lab 10 & & & Lab 11 & & \\
\hline & mean & stdev & rsd & mean & stdev & rsd & mean & stdev & rsd & mean & stdev & rsd & mean & stdev & rsd & mean & stdev & rsd \\
\hline density $(\mathrm{g} / \mathrm{mL})$ & 1.04 & 0.00 & $0.14 \%$ & 0.995 & 0.016 & $1.60 \%$ & 1.00 & 0.00 & $0.01 \%$ & \multicolumn{2}{|c|}{ not reported use 1} & & & & & 0.983 & 0.006 & $0.64 \%$ \\
\hline \multirow{2}{*}{\multicolumn{19}{|c|}{ CONVERT ALL TO $\mu \mathrm{mol} / \mathrm{L}$}} \\
\hline & & & & & & & & & & & & & & & & & & \\
\hline & Lab 6 & & & Lab 7 & & & Lab 8 & & & Lab 9 & & & Lab 10 & & & Lab 11 & & \\
\hline & mean & stdev & rsd & mean & stdev & rsd & mean & stdev & rsd & mean & stdev & rsd & mean & stdev & rsd & mean & stdev & rsd \\
\hline C14:0 & 142 & 3 & $2.11 \%$ & 152 & 15 & $9.60 \%$ & 19.9 & 1.0 & $4.80 \%$ & 150 & 5 & $3.20 \%$ & 86.4 & 0.8 & $0.88 \%$ & 152 & 1 & $0.63 \%$ \\
\hline C14:1n5 & NA & & & 15.0 & 1.3 & $8.95 \%$ & $<L O D$ & & & 12.1 & 0.8 & $6.86 \%$ & 5.49 & 0.62 & $11.35 \%$ & 9.24 & 0.16 & $1.68 \%$ \\
\hline C16:0 & 2963 & 87 & $2.95 \%$ & 2782 & 329 & $11.81 \%$ & 299 & 16 & $5.24 \%$ & 2995 & 122 & $4.07 \%$ & 2057 & 15 & $0.72 \%$ & 3151 & 20 & $0.62 \%$ \\
\hline C16:1n7 & 266 & 7 & $2.56 \%$ & 63.9 & 6.7 & $10.47 \%$ & 8.08 & 0.81 & $10.07 \%$ & 258 & 11 & $4.20 \%$ & 182 & 3 & $1.80 \%$ & 264 & 4 & $1.48 \%$ \\
\hline C18:0 & 905 & 12 & $1.33 \%$ & 997 & 94 & $9.47 \%$ & 102 & 10 & $9.86 \%$ & 928 & 44 & $4.69 \%$ & 696 & 4 & $0.64 \%$ & 808 & 3 & $0.38 \%$ \\
\hline C18:1n7 & 146 & 0 & $0.00 \%$ & 118 & 11 & $9.13 \%$ & 36.2 & 2.4 & $6.55 \%$ & NA & & & 130 & 8 & $6.06 \%$ & 140 & 1 & $0.90 \%$ \\
\hline C18:1n9 & 2680 & 85 & $3.19 \%$ & 2473 & 308 & $12.45 \%$ & 127 & 7 & $5.79 \%$ & 2927 & 103 & $3.51 \%$ & 1984 & 23 & $1.17 \%$ & 2764 & 23 & $0.84 \%$ \\
\hline C18:2n6 & 4353 & 172 & $3.96 \%$ & 3180 & 354 & $11.12 \%$ & 100 & 6 & $6.44 \%$ & 4354 & 147 & $3.38 \%$ & 3200 & 40 & $1.24 \%$ & 4479 & 37 & $0.81 \%$ \\
\hline C18:3n3 & 108 & 2 & $1.85 \%$ & 107 & 17 & $15.80 \%$ & $<L O D$ & & & 112 & 6 & $5.36 \%$ & 78.7 & 0.7 & $0.85 \%$ & 118 & 1 & $1.25 \%$ \\
\hline C18:3n6 & 74.3 & 1.2 & $1.62 \%$ & 54.5 & 8.1 & $14.92 \%$ & 5.47 & 0.29 & $5.26 \%$ & 76.1 & 2.7 & $3.54 \%$ & 54.4 & 1.1 & $2.07 \%$ & 76.3 & 0.7 & $0.87 \%$ \\
\hline C20:0 & NA & & & 25.0 & 3.6 & $14.25 \%$ & $<L O D$ & & & 34.0 & 5.2 & $15.29 \%$ & 26.1 & 0.4 & $1.64 \%$ & 29.4 & 0.3 & $0.84 \%$ \\
\hline C20:1n9 & NA & & & 37.3 & 12.2 & $32.85 \%$ & $<L O D$ & & & 14.4 & 1.7 & $11.95 \%$ & 12.2 & 0.4 & $3.13 \%$ & 16.0 & 0.3 & $1.96 \%$ \\
\hline $\mathrm{C} 20: 2 \mathrm{n6}$ & NA & & & 5.98 & 1.65 & $27.55 \%$ & $<L O D$ & & & 24.8 & 1.5 & $5.92 \%$ & 19.4 & 0.5 & $2.82 \%$ & 23.1 & 0.2 & $0.87 \%$ \\
\hline $\mathrm{C} 20: 3 \mathrm{n} 6$ & 189 & 3 & $1.52 \%$ & 209 & 23 & $11.06 \%$ & 7.94 & 0.50 & $6.32 \%$ & 190 & 11 & $5.62 \%$ & 156 & 2 & $1.25 \%$ & 196 & 7 & $3.35 \%$ \\
\hline C20:4n6 & 823 & 17 & $2.07 \%$ & 780 & 85 & $10.89 \%$ & 33.9 & 1.8 & $5.20 \%$ & 813 & 49 & $6.03 \%$ & 646 & 9 & $1.42 \%$ & 856 & 8 & $0.94 \%$ \\
\hline $\mathrm{C} 20: 5 \mathrm{n} 3$ & 68.5 & 1.1 & $1.55 \%$ & 68.0 & 6.1 & $8.95 \%$ & $<L O D$ & & & 67.4 & 5.1 & $7.59 \%$ & 62.6 & 0.2 & $0.30 \%$ & 71.9 & 0.5 & $0.73 \%$ \\
\hline $\mathrm{C} 22: 0$ & 69.7 & 1.9 & $2.66 \%$ & 2.37 & 0.21 & $8.89 \%$ & $\angle L O D$ & & & 66.7 & 11.0 & $16.55 \%$ & 88.6 & 1.6 & $1.85 \%$ & 92.7 & 1.2 & $1.30 \%$ \\
\hline C22:1n9 & NA & & & & & & $<L O D$ & & & 16.3 & 3.3 & $20.05 \%$ & 2.20 & 0.31 & $14.02 \%$ & 4.46 & 0.32 & $7.19 \%$ \\
\hline C22:4n6 & 21.3 & 0.8 & $3.79 \%$ & 27.3 & 2.5 & $9.24 \%$ & $<L O D$ & & & 41.8 & 2.2 & $5.23 \%$ & 20.2 & 0.5 & $2.55 \%$ & 23.0 & 1.3 & $5.81 \%$ \\
\hline C22:5n3 & 48.3 & 1.1 & $2.28 \%$ & 53.5 & 8.1 & $15.16 \%$ & $\angle L O D$ & & & 46.4 & 2.8 & $6.03 \%$ & 44.3 & 0.3 & $0.69 \%$ & 52.1 & 1.2 & $2.35 \%$ \\
\hline C22:5n6 & NA & & & 16.7 & 3.5 & $21.13 \%$ & $<L O D$ & & & 13.3 & 3.6 & $26.73 \%$ & 14.5 & 0.3 & $2.35 \%$ & 16.1 & 0.5 & $3.14 \%$ \\
\hline $\mathrm{C} 22: 6 \mathrm{n} 3$ & 156 & 3 & $1.85 \%$ & 195 & 16 & $8.45 \%$ & 4.53 & 0.61 & $13.44 \%$ & 160 & 10 & $6.27 \%$ & 136 & 2 & $1.31 \%$ & 179 & 1 & $0.33 \%$ \\
\hline $\mathrm{C} 24: 0$ & 58.3 & 2.5 & $4.23 \%$ & 2.61 & 0.18 & $6.73 \%$ & $<L O D$ & & & 54.3 & 9.7 & $17.87 \%$ & 81.9 & 2.7 & $3.29 \%$ & 78.0 & 1.0 & $1.33 \%$ \\
\hline \multirow[t]{5}{*}{ C24:1n9 } & 61.6 & 3.4 & $5.44 \%$ & & & & $<L O D$ & & & 52.9 & 10.1 & $19.12 \%$ & 84.4 & 1.7 & $1.99 \%$ & 86.4 & 2.6 & $2.95 \%$ \\
\hline & & & & & & & & & & \multicolumn{2}{|c|}{ NOTE for Lab 9: } & & & & & & & \\
\hline & & & & & & & & & & C18:1n9 & Oleic acid & & \multicolumn{4}{|c|}{ Total 18:1 determined \& entered } & & \\
\hline & & & & & & & & & & C20:1n9 & \multicolumn{2}{|c|}{ 11-Eicosenoic acid } & \multicolumn{4}{|c|}{ Total 20:1 determined \& entered } & & \\
\hline & & & & & & & & & & C22:1n9 & \multicolumn{2}{|c|}{ Docosenoic acid } & \multicolumn{4}{|c|}{ Total 22:1 determined \& entered } & & \\
\hline
\end{tabular}




\begin{tabular}{|c|c|c|c|c|c|c|c|c|c|c|c|c|c|c|c|}
\hline \multicolumn{16}{|c|}{ Table 4. Interlaboratory data received for SRM 2378-3 } \\
\hline & & & & & & & & & & & & & & & \\
\hline \multirow[t]{3}{*}{ units reported } & $\mu \mathrm{g} / \mathrm{g}$ & & & $\mu \mathrm{mol} / \mathrm{L}$ & & & $\mu \mathrm{mol} / \mathrm{L}$ & & & $\mu \mathrm{mol} / \mathrm{L}$ & & & $\mu \mathrm{g} / \mathrm{g}$ & & \\
\hline & Lab 1 & & & Lab 2 & & & Lab 3 & & & Lab 4 & & & Lab 5 & & \\
\hline & mean & stdev & rsd & mean & stdev & rsd & mean & stdev & rsd & mean & stdev & rsd & mean & stdev & rsd \\
\hline C14:0 & 34.1 & 2.0 & $5.75 \%$ & 158 & 3 & $1.60 \%$ & 163 & 2 & $1.52 \%$ & 129 & 6 & $4.58 \%$ & 40.1 & 0.7 & $1.84 \%$ \\
\hline C14:1n5 & 4.02 & 0.29 & $7.31 \%$ & 19.3 & 0.6 & $2.99 \%$ & NA & & & & & & 2.81 & 0.18 & $6.41 \%$ \\
\hline C16:0 & 774 & 11 & $1.47 \%$ & 1307 & 29 & $2.21 \%$ & 2401 & 28 & $1.16 \%$ & 2495 & 141 & $5.66 \%$ & 780 & 7 & $0.83 \%$ \\
\hline C16:1n7 & 49.4 & 1.7 & $3.51 \%$ & 174 & 8 & $4.83 \%$ & 180 & 2 & $1.13 \%$ & 175 & 5 & $3.02 \%$ & 56.7 & 0.5 & $0.79 \%$ \\
\hline C18:0 & 207 & 10 & $4.88 \%$ & 663 & 10 & $1.44 \%$ & 742 & 8 & $1.08 \%$ & 736 & 31 & $4.26 \%$ & 265 & 2 & $0.87 \%$ \\
\hline C18:1n7 & 36.3 & 3.2 & $8.94 \%$ & 212 & 5 & $2.33 \%$ & 92.5 & 2.4 & $2.59 \%$ & NA & & & 51.3 & 0.7 & $1.36 \%$ \\
\hline C18:1n9 & 603 & 16 & $2.57 \%$ & 1077 & 61 & $5.68 \%$ & 1841 & 26 & $1.43 \%$ & 1769 & 108 & $6.12 \%$ & 649 & 7 & $1.11 \%$ \\
\hline C18:2n6 & 931 & 27 & $2.91 \%$ & 3327 & 107 & $3.21 \%$ & 3282 & 53 & $1.63 \%$ & 2689 & 200 & $7.44 \%$ & 1103 & 6 & $0.53 \%$ \\
\hline C18:3n3 & 16.9 & 0.2 & $1.36 \%$ & 61.0 & 1.7 & $2.84 \%$ & 51.6 & 1.1 & $2.20 \%$ & 40.8 & 2.4 & $5.89 \%$ & 19.3 & 0.4 & $1.87 \%$ \\
\hline C18:3n6 & 15.1 & 0.4 & $2.41 \%$ & 50.3 & 0.6 & $1.15 \%$ & 46.6 & 1.8 & $3.86 \%$ & 41.9 & 1.9 & $4.44 \%$ & 17.8 & 0.4 & $2.24 \%$ \\
\hline C20:0 & 10.1 & 0.2 & $1.57 \%$ & 59.0 & 1.0 & $1.69 \%$ & 30.6 & 4.1 & $13.29 \%$ & 12.5 & 1.8 & $14.17 \%$ & 6.06 & 0.27 & $4.41 \%$ \\
\hline C20:1n9 & 5.49 & 0.09 & $1.64 \%$ & NA & & & 20.7 & 1.4 & $6.73 \%$ & 14.4 & 1.9 & $13.24 \%$ & 5.62 & 0.30 & $5.34 \%$ \\
\hline C20:2n6 & NA & & & NA & & & 20.5 & 0.4 & $2.11 \%$ & 16.6 & 1.8 & $10.98 \%$ & 6.79 & 0.12 & $1.77 \%$ \\
\hline C20:3n6 & NA & & & 62.0 & 1.0 & $1.61 \%$ & 126 & 2 & $1.31 \%$ & 102 & 7 & $6.42 \%$ & 43.5 & 0.6 & $1.47 \%$ \\
\hline C20:4n6 & 245 & 12 & $5.10 \%$ & 1127 & 12 & $1.02 \%$ & 691 & 13 & $1.89 \%$ & 596 & 36 & $5.97 \%$ & 264 & 2 & $0.76 \%$ \\
\hline$C 20: 5 n 3$ & 21.0 & 0.5 & $2.61 \%$ & 87.0 & 7.2 & $8.29 \%$ & 59.8 & 3.2 & $5.40 \%$ & 61.4 & 2.2 & $3.63 \%$ & 21.5 & 0.5 & $2.10 \%$ \\
\hline C22:0 & 14.8 & 0.3 & $2.02 \%$ & 42.2 & 1.0 & $2.34 \%$ & 20.7 & 5.0 & $24.11 \%$ & 28.0 & 2.2 & $7.97 \%$ & 14.6 & 0.9 & $5.83 \%$ \\
\hline C22:1n9 & 2.83 & 0.07 & $2.36 \%$ & 6.00 & 0.00 & $0.00 \%$ & NA & & & NA & & & 3.07 & 0.07 & $2.28 \%$ \\
\hline C22:4n6 & NA & & & 12.3 & 0.6 & $4.68 \%$ & 19.7 & 2.7 & $13.82 \%$ & 13.1 & 0.5 & $3.62 \%$ & 4.51 & 0.46 & $10.08 \%$ \\
\hline$C 22: 5 n 3$ & 10.5 & 0.1 & $0.63 \%$ & 28.7 & 0.6 & $2.01 \%$ & 36.7 & 1.3 & $3.67 \%$ & 27.0 & 2.1 & $7.96 \%$ & 12.7 & 1.0 & $8.19 \%$ \\
\hline C22:5n6 & NA & & & 8.67 & 0.58 & $6.66 \%$ & 12.7 & 2.6 & $20.27 \%$ & 11.5 & 2.4 & $20.79 \%$ & 4.84 & 0.05 & $1.05 \%$ \\
\hline$C 22: 6 n 3$ & 51.1 & 0.6 & $1.13 \%$ & 157 & 7 & $4.18 \%$ & 173 & 2 & $1.03 \%$ & 124 & 3 & $2.04 \%$ & 59.9 & 0.4 & $0.70 \%$ \\
\hline C24:0 & 12.5 & 0.1 & $0.70 \%$ & 43.4 & 1.0 & $2.32 \%$ & 16.5 & 3.4 & $20.60 \%$ & 16.3 & 1.2 & $7.33 \%$ & 11.0 & 0.6 & $5.55 \%$ \\
\hline C24:1n9 & 13.6 & 0.9 & $6.60 \%$ & 48.3 & 2.1 & $4.31 \%$ & 15.9 & 1.0 & $6.39 \%$ & 13.1 & 1.9 & $14.58 \%$ & 14.2 & 0.4 & $2.82 \%$ \\
\hline
\end{tabular}

\begin{tabular}{|c|c|c|c|c|c|c|c|c|c|c|c|c|c|c|c|c|c|c|}
\hline \multicolumn{19}{|c|}{ Table 4 (continued) Interlaboratory data received for SRM 2378-3 } \\
\hline & & & & & & & & & & & & & & & & & & \\
\hline \multirow[t]{3}{*}{ units reported } & $\mu \mathrm{mol} / \mathrm{L}$ & & & $\mu \mathrm{g} / \mathrm{g}$ & & & $\mu \mathrm{mol} / \mathrm{L}$ & & & $\mu \mathrm{g} / \mathrm{g}$ & & & $\mu \mathrm{mol} / \mathrm{L}$ & & & $\mu \mathrm{mol} / \mathrm{L}$ & & \\
\hline & Lab 6 & & & Lab 7 & & & Lab 8 & & & Lab 9 & & & Lab 10 & & & Lab 11 & & \\
\hline & mean & stdev & rsd & mean & stdev & rsd & mean & stdev & rsd & mean & stdev & rsd & mean & stdev & rsd & mean & stdev & rsd \\
\hline C14:0 & 149 & 3 & $1.69 \%$ & 51.7 & 1.5 & $2.98 \%$ & 22.9 & 1.9 & $8.24 \%$ & 35.8 & 2.3 & $6.36 \%$ & 88.2 & 1.2 & $1.40 \%$ & 157 & 2 & $1.12 \%$ \\
\hline C14:1n5 & NA & & & 5.62 & 0.72 & $12.73 \%$ & $<L O D$ & & & 2.82 & 0.09 & $3.14 \%$ & 5.69 & 0.28 & $4.97 \%$ & 10.4 & 0.1 & $0.79 \%$ \\
\hline C16:0 & 2473 & 67 & $2.69 \%$ & 827 & 18 & $2.22 \%$ & 300 & 19 & $6.22 \%$ & 644 & 27 & $4.27 \%$ & 1719 & 15 & $0.85 \%$ & 2592 & 23 & $0.88 \%$ \\
\hline C16:1n7 & 181 & 5 & $2.49 \%$ & 15.1 & 0.5 & $3.44 \%$ & 8.23 & 1.02 & $12.35 \%$ & 44.7 & 1.9 & $4.36 \%$ & 120 & 3 & $2.30 \%$ & 177 & 0 & $0.27 \%$ \\
\hline C18:0 & 781 & 10 & $1.26 \%$ & 308 & 5 & $1.48 \%$ & 93.6 & 12.5 & $13.32 \%$ & 221 & 11 & $4.75 \%$ & 587 & 7 & $1.24 \%$ & 657 & 12 & $1.81 \%$ \\
\hline C18:1n7 & 118 & 2 & $1.69 \%$ & 34.9 & 12.6 & $36.00 \%$ & 39.6 & 2.7 & $6.81 \%$ & ND & & & 107 & 4 & $3.77 \%$ & 110 & 1 & $0.95 \%$ \\
\hline C18:1n9 & 1927 & 40 & $2.10 \%$ & 744 & 21 & $2.85 \%$ & 101 & 7 & $7.13 \%$ & 585 & 31 & $5.31 \%$ & 1386 & 4 & $0.31 \%$ & 1938 & 16 & $0.82 \%$ \\
\hline C18:2n6 & 3343 & 121 & $3.61 \%$ & 1128 & 50 & $4.43 \%$ & 115 & 9 & $7.94 \%$ & 906 & 47 & $5.16 \%$ & 2331 & 16 & $0.68 \%$ & 3328 & 21 & $0.64 \%$ \\
\hline $\mathrm{C} 18: 3 \mathrm{n} 3$ & 57.8 & 1.4 & $2.40 \%$ & 23.9 & 1.6 & $6.88 \%$ & $<\angle O D$ & & & 17.4 & 0.6 & $3.49 \%$ & 41.3 & 0.2 & $0.59 \%$ & 62.6 & 0.6 & $0.93 \%$ \\
\hline C18:3n6 & 50.3 & 1.6 & $3.08 \%$ & 18.7 & 0.8 & $4.16 \%$ & $<\angle O D$ & & & 14.4 & 0.9 & $6.25 \%$ & 34.0 & 0.1 & $0.38 \%$ & 51.3 & 0.7 & $1.35 \%$ \\
\hline $\mathrm{C} 20: 0$ & NA & & & 10.3 & 0.5 & $4.93 \%$ & $<L O D$ & & & 8.64 & 1.80 & $20.81 \%$ & 19.2 & 0.4 & $1.88 \%$ & 21.2 & 0.4 & $1.87 \%$ \\
\hline C20:1n9 & NA & & & 9.16 & 3.54 & $38.65 \%$ & $<L O D$ & & & 4.10 & 0.07 & $1.59 \%$ & 13.1 & 0.3 & $2.02 \%$ & 16.8 & 0.1 & $0.57 \%$ \\
\hline $\mathrm{C} 20: 2 \mathrm{n} 6$ & NA & & & 2.06 & 0.43 & $20.84 \%$ & $<\angle O D$ & & & 6.62 & 0.31 & $4.72 \%$ & 16.0 & 0.3 & $2.00 \%$ & 19.6 & 0.1 & $0.61 \%$ \\
\hline $\mathrm{C} 20: 3 \mathrm{n} 6$ & 117 & 2 & $1.31 \%$ & 54.6 & 1.5 & $2.67 \%$ & 5.04 & 0.29 & $5.73 \%$ & 36 & 1 & $3.78 \%$ & 95.4 & 1.2 & $1.31 \%$ & 121 & 0 & $0.29 \%$ \\
\hline C20:4n6 & 799 & 17 & $2.07 \%$ & 322 & 11 & $3.45 \%$ & 32.1 & 2.0 & $6.10 \%$ & 222 & 11 & $4.81 \%$ & 569 & 3 & $0.60 \%$ & 768 & 7 & $0.92 \%$ \\
\hline $\mathrm{C} 20: 5 \mathrm{n} 3$ & 62.9 & 1.0 & $1.66 \%$ & 26.6 & 0.6 & $2.06 \%$ & $<L O D$ & & & 18.1 & 0.7 & $4.05 \%$ & 50.8 & 0.7 & $1.35 \%$ & 62.2 & 1.0 & $1.65 \%$ \\
\hline C22:0 & 47.0 & 1.1 & $2.37 \%$ & 1.22 & 0.64 & $52.34 \%$ & $<\angle O D$ & & & 15.9 & 0.2 & $1.25 \%$ & 59.8 & 0.9 & $1.55 \%$ & 61.0 & 0.2 & $0.29 \%$ \\
\hline C22:1n9 & NA & & & & & & $<\angle O D$ & & & 6.74 & 1.07 & $15.83 \%$ & 2.09 & 0.08 & $3.79 \%$ & 5.03 & 0.51 & $10.20 \%$ \\
\hline C22:4n6 & 17.0 & 0.2 & $1.36 \%$ & 9.91 & 0.10 & $0.96 \%$ & $<\angle O D$ & & & 10.8 & 1.2 & $10.78 \%$ & 16.1 & 0.2 & $1.17 \%$ & 18.6 & 0.3 & $1.78 \%$ \\
\hline C22:5n3 & 33.1 & 0.7 & $2.09 \%$ & 16.9 & 0.9 & $5.35 \%$ & $<L O D$ & & & 10.24 & 0.36 & $3.48 \%$ & 29.3 & 0.6 & $2.05 \%$ & 36.4 & 1.1 & $2.98 \%$ \\
\hline $\mathrm{C} 22: 5 \mathrm{n} 6$ & NA & & & 7.06 & 0.41 & $5.78 \%$ & $<L O D$ & & & 4.57 & 0.55 & $12.02 \%$ & 13.8 & 0.5 & $3.48 \%$ & 16.0 & 0.5 & $3.28 \%$ \\
\hline C22:6n3 & 157 & 3 & $1.69 \%$ & 83.6 & 3.2 & $3.85 \%$ & 5.89 & 0.35 & $5.89 \%$ & 51.6 & 2.7 & $5.19 \%$ & 134 & 0 & $0.35 \%$ & 177 & 2 & $1.27 \%$ \\
\hline C24:0 & 39.5 & 0.4 & $1.10 \%$ & 1.53 & 0.28 & $18.51 \%$ & $<L O D$ & & & 15.2 & 0.8 & $5.03 \%$ & 56.5 & 1.8 & $3.13 \%$ & 53.2 & 0.3 & $0.58 \%$ \\
\hline \multirow[t]{5}{*}{$\mathrm{C} 24: 1 \mathrm{n} 9$} & 43.6 & 1.8 & $4.05 \%$ & & & & $<L O D$ & & & 15.4 & 0.3 & $1.90 \%$ & 63.8 & 1.3 & $2.05 \%$ & 63.5 & 1.1 & $1.68 \%$ \\
\hline & & & & & & & & & & \multicolumn{2}{|c|}{ NOTE for Lab 9: } & & \multirow{2}{*}{\multicolumn{4}{|c|}{ Total 18:1 determined \& entered }} & & \\
\hline & & & & & & & & & & C18:1n9 & Oleic acid & & & & & & & \\
\hline & & & & & & & & & & C20:1n9 & \multicolumn{2}{|c|}{ 11-Eicosenoic acid } & \multicolumn{4}{|c|}{ Total 20:1 determined \& entered } & & \\
\hline & & & & & & & & & & C22:1n9 & \multicolumn{2}{|c|}{ Docosenoic acid } & \multicolumn{4}{|c|}{ Total 22:1 determined \& entered } & & \\
\hline
\end{tabular}




\begin{tabular}{|c|c|c|c|c|c|c|c|c|c|c|c|c|c|c|c|}
\hline \multicolumn{16}{|c|}{ Table 4 (continued) Interlaboratory data received for SRM 2378-3 } \\
\hline & Lab 1 & \multirow{3}{*}{$\begin{array}{c}\text { stdev } \\
0.00\end{array}$} & \multirow{3}{*}{$\begin{array}{c}\text { rsd } \\
0.00 \%\end{array}$} & Lab 2 & & \multirow[b]{2}{*}{ rsd } & \multirow{3}{*}{$\begin{array}{c}\text { Lab } 3 \\
\text { mean } \\
1.08\end{array}$} & \multirow{3}{*}{$\begin{array}{c}\text { stdev } \\
0.02\end{array}$} & \multirow{3}{*}{$\begin{array}{c}\text { rsd } \\
1.86 \%\end{array}$} & Lab 4 & & \multirow[b]{2}{*}{ rsd } & Lab 5 & \multirow{3}{*}{$\begin{array}{c}\text { stdev } \\
0.01\end{array}$} & \multirow{3}{*}{$\begin{array}{c}\text { rsd } \\
0.56 \%\end{array}$} \\
\hline & mean & & & mean & stdev & & & & & mean & stdev & & mean & & \\
\hline density $(\mathrm{g} / \mathrm{mL})$ & 1.02 & & & \multicolumn{2}{|c|}{ not reported } & & & & & \multicolumn{2}{|c|}{ not reported } & & 1.02 & & \\
\hline & & & & & & & & & & & & & & & \\
\hline \multicolumn{16}{|c|}{ CONVERT ALL TO $\mu \mathrm{mol} / \mathrm{L}$} \\
\hline & Lab 1 & & & Lab 2 & & & Lab 3 & & & Lab 4 & & & Lab 5 & & \\
\hline & mean & stdev & rsd & mean & stdev & rsd & mean & stdev & rsd & mean & stdev & rsd & mean & stdev & rsd \\
\hline C14:0 & 152 & 9 & $5.75 \%$ & 158 & 3 & $1.60 \%$ & 163 & 2 & $1.52 \%$ & 129 & 6 & $4.58 \%$ & 180 & 3 & $1.84 \%$ \\
\hline C14:1n5 & 18.2 & 1.3 & $7.31 \%$ & 19.3 & 0.6 & $2.99 \%$ & NA & & & & & & 12.7 & 0.8 & $6.41 \%$ \\
\hline C16:0 & 3085 & 45 & $1.47 \%$ & 1307 & 29 & $2.21 \%$ & 2401 & 28 & $1.16 \%$ & 2495 & 141 & $5.66 \%$ & 3113 & 26 & $0.83 \%$ \\
\hline C16:1n7 & 199 & 7 & $3.51 \%$ & 174 & 8 & $4.83 \%$ & 180 & 2 & $1.13 \%$ & 175 & 5 & $3.02 \%$ & 228 & 2 & $0.79 \%$ \\
\hline C18:0 & 745 & 36 & $4.88 \%$ & 663 & 10 & $1.44 \%$ & 742 & 8 & $1.08 \%$ & 736 & 31 & $4.26 \%$ & 953 & 8 & $0.87 \%$ \\
\hline C18:1n7 & 131 & 12 & $8.94 \%$ & 212 & 5 & $2.33 \%$ & 92.5 & 2.4 & $2.59 \%$ & NA & & & 186 & 3 & $1.36 \%$ \\
\hline C18:1n9 & 2183 & 56 & $2.57 \%$ & 1077 & 61 & $5.68 \%$ & 1841 & 26 & $1.43 \%$ & 1769 & 108 & $6.12 \%$ & 2351 & 26 & $1.11 \%$ \\
\hline C18:2n6 & 3393 & 99 & $2.91 \%$ & 3327 & 107 & $3.21 \%$ & 3282 & 53 & $1.63 \%$ & 2689 & 200 & $7.44 \%$ & 4024 & 21 & $0.53 \%$ \\
\hline C18:3n3 & 62.2 & 0.8 & $1.36 \%$ & 61.0 & 1.7 & $2.84 \%$ & 51.6 & 1.1 & $2.20 \%$ & 40.8 & 2.4 & $5.89 \%$ & 70.9 & 1.3 & $1.87 \%$ \\
\hline C18:3n6 & 55.5 & 1.3 & $2.41 \%$ & 50.3 & 0.6 & $1.15 \%$ & 46.6 & 1.8 & $3.86 \%$ & 41.9 & 1.9 & $4.44 \%$ & 65.5 & 1.5 & $2.24 \%$ \\
\hline C2O:0 & 33.1 & 0.5 & $1.57 \%$ & 59.0 & 1.0 & $1.69 \%$ & 30.6 & 4.1 & $13.29 \%$ & 12.5 & 1.8 & $14.17 \%$ & 19.8 & 0.9 & $4.41 \%$ \\
\hline C20:1n9 & 18.1 & 0.3 & $1.64 \%$ & NA & & & 20.7 & 1.4 & $6.73 \%$ & 14.4 & 1.9 & $13.24 \%$ & 18.5 & 1.0 & $5.34 \%$ \\
\hline C20:2n6 & NA & & & NA & & & 20.5 & 0.4 & $2.11 \%$ & 16.6 & 1.8 & $10.98 \%$ & 22.5 & 0.4 & $1.77 \%$ \\
\hline C20:3n6 & NA & & & 62.0 & 1.0 & $1.61 \%$ & 126 & 2 & $1.31 \%$ & 102 & 7 & $6.42 \%$ & 145 & 2 & $1.47 \%$ \\
\hline C20:4n6 & 822 & 42 & $5.10 \%$ & 1127 & 12 & $1.02 \%$ & 691 & 13 & $1.89 \%$ & 596 & 36 & $5.97 \%$ & 887 & 7 & $0.76 \%$ \\
\hline C20:5n3 & 70.9 & 1.8 & $2.61 \%$ & 87.0 & 7.2 & $8.29 \%$ & 59.8 & 3.2 & $5.40 \%$ & 61.4 & 2.2 & $3.63 \%$ & 72.6 & 1.5 & $2.10 \%$ \\
\hline $\mathrm{C} 22: 0$ & 44.4 & 0.9 & $2.02 \%$ & 42.2 & 1.0 & $2.34 \%$ & 20.7 & 5.0 & $24.11 \%$ & 28.0 & 2.2 & $7.97 \%$ & 43.8 & 2.6 & $5.83 \%$ \\
\hline C22:1n9 & 8.53 & 0.20 & $2.36 \%$ & 6.00 & 0.00 & $0.00 \%$ & NA & & & NA & & & 9.28 & 0.21 & $2.28 \%$ \\
\hline C22:4n6 & NA & & & 12.3 & 0.6 & $4.68 \%$ & 19.7 & 2.7 & $13.82 \%$ & 13.1 & 0.5 & $3.62 \%$ & 13.9 & 1.4 & $10.08 \%$ \\
\hline C22:5n3 & 32.3 & 0.2 & $0.63 \%$ & 28.7 & 0.6 & $2.01 \%$ & 36.7 & 1.3 & $3.67 \%$ & 27.0 & 2.1 & $7.96 \%$ & 39.3 & 3.2 & $8.19 \%$ \\
\hline C22:5n6 & NA & & & 8.67 & 0.58 & $6.66 \%$ & 12.7 & 2.6 & $20.27 \%$ & 11.5 & 2.4 & $20.79 \%$ & 15.0 & 0.2 & $1.05 \%$ \\
\hline $\mathrm{C} 22: 6 \mathrm{n} 3$ & 159 & 2 & $1.13 \%$ & 157 & 7 & $4.18 \%$ & 173 & 2 & $1.03 \%$ & 124 & 3 & $2.04 \%$ & 186 & 1 & $0.70 \%$ \\
\hline C24:0 & 34.7 & 0.2 & $0.70 \%$ & 43.4 & 1.0 & $2.32 \%$ & 16.5 & 3.4 & $20.60 \%$ & 16.3 & 1.2 & $7.33 \%$ & 30.5 & 1.7 & $5.55 \%$ \\
\hline C24:1n9 & 37.9 & 2.5 & $6.60 \%$ & 48.3 & 2.1 & $4.31 \%$ & 15.9 & 1.0 & $6.39 \%$ & 13.1 & 1.9 & $14.58 \%$ & 39.5 & 1.1 & $2.82 \%$ \\
\hline
\end{tabular}

\begin{tabular}{|c|c|c|c|c|c|c|c|c|c|c|c|c|c|c|c|c|c|c|}
\hline \multicolumn{19}{|c|}{ Table 4 (continued) Interlaboratory data received for SRM 2378-3 } \\
\hline & Lab 6 & & & Lab 7 & & & Lab 8 & & & Lab 9 & & & Lab 10 & & & Lab 11 & & \\
\hline & mean & stdev & rsd & mean & stdev & rsd & mean & stdev & rsd & mean & stdev & rsd & mean & stdev & rsd & mean & stdev & rsd \\
\hline density $(\mathrm{g} / \mathrm{mL})$ & 1.04 & 0.00 & $0.47 \%$ & 0.988 & 0.010 & $1.00 \%$ & 1.02 & 0.00 & $0.01 \%$ & \multicolumn{2}{|c|}{ not reported use 1} & & & & & 0.987 & 0.001 & $0.06 \%$ \\
\hline \multirow{2}{*}{\multicolumn{19}{|c|}{ CONVERT ALL TO $\mu \mathrm{mol} / \mathrm{L}$}} \\
\hline & & & & & & & & & & & & & & & & & & \\
\hline & Lab 6 & & & Lab 7 & & & Lab 8 & & & Lab 9 & & & Lab 10 & & & Lab 11 & & \\
\hline & mean & stdev & rsd & mean & stdev & rsd & mean & stdev & rsd & mean & stdev & rsd & mean & stdev & rsd & mean & stdev & rsd \\
\hline C14:0 & 149 & 3 & $1.69 \%$ & 224 & 7 & $2.98 \%$ & 22.9 & 1.9 & $8.24 \%$ & 157 & 10 & $6.36 \%$ & 88.2 & 1.2 & $1.40 \%$ & 157 & 2 & $1.12 \%$ \\
\hline C14:1n5 & NA & & & 24.5 & 3.1 & $12.73 \%$ & $<L O D$ & & & 12.4 & 0.4 & $3.14 \%$ & 5.69 & 0.28 & $4.97 \%$ & 10.4 & 0.1 & $0.79 \%$ \\
\hline C16:0 & 2473 & 67 & $2.69 \%$ & 3186 & 71 & $2.22 \%$ & 300 & 19 & $6.22 \%$ & 2511 & 107 & $4.27 \%$ & 1719 & 15 & $0.85 \%$ & 2592 & 23 & $0.88 \%$ \\
\hline C16:1n7 & 181 & 5 & $2.49 \%$ & 58.6 & 2.0 & $3.44 \%$ & 8.23 & 1.02 & $12.35 \%$ & 176 & 8 & $4.36 \%$ & 120 & 3 & $2.30 \%$ & 177 & 0 & $0.27 \%$ \\
\hline C18:0 & 781 & 10 & $1.26 \%$ & 1069 & 16 & $1.48 \%$ & 93.6 & 12.5 & $13.32 \%$ & 777 & 37 & $4.75 \%$ & 587 & 7 & $1.24 \%$ & 657 & 12 & $1.81 \%$ \\
\hline C18:1n7 & 118 & 2 & $1.69 \%$ & 122 & 44 & $36.00 \%$ & 39.6 & 2.7 & $6.81 \%$ & NA & & & 107 & 4 & $3.77 \%$ & 110 & 1 & $0.95 \%$ \\
\hline C18:1n9 & 1927 & 40 & $2.10 \%$ & 2603 & 74 & $2.85 \%$ & 101 & 7 & $7.13 \%$ & 2071 & 110 & $5.31 \%$ & 1386 & 4 & $0.31 \%$ & 1938 & 16 & $0.82 \%$ \\
\hline C18:2n6 & 3343 & 121 & $3.61 \%$ & 3973 & 176 & $4.43 \%$ & 115 & 9 & $7.94 \%$ & 3231 & 167 & $5.16 \%$ & 2331 & 16 & $0.68 \%$ & 3328 & 21 & $0.64 \%$ \\
\hline C18:3n3 & 57.8 & 1.4 & $2.40 \%$ & 84.6 & 5.8 & $6.88 \%$ & $<L O D$ & & & 62.6 & 2.2 & $3.49 \%$ & 41.3 & 0.2 & $0.59 \%$ & 62.6 & 0.6 & $0.93 \%$ \\
\hline C18:3n6 & 50.3 & 1.6 & $3.08 \%$ & 66.2 & 2.8 & $4.16 \%$ & $<L O D$ & & & 51.7 & 3.2 & $6.25 \%$ & 34.0 & 0.1 & $0.38 \%$ & 51.3 & 0.7 & $1.35 \%$ \\
\hline C20:0 & NA & & & 32.4 & 1.6 & $4.93 \%$ & $<L O D$ & & & 27.6 & 5.8 & $20.81 \%$ & 19.2 & 0.4 & $1.88 \%$ & 21.2 & 0.4 & $1.87 \%$ \\
\hline C20:1n9 & NA & & & 29.1 & 11.3 & $38.65 \%$ & $<L O D$ & & & 13.2 & 0.2 & $1.59 \%$ & 13.1 & 0.3 & $2.02 \%$ & 16.8 & 0.1 & $0.57 \%$ \\
\hline $\mathrm{C} 20: 2 \mathrm{n} 6$ & NA & & & 6.59 & 1.37 & $20.84 \%$ & $<L O D$ & & & 21.5 & 1.0 & $4.72 \%$ & 16.0 & 0.3 & $2.00 \%$ & 19.6 & 0.1 & $0.61 \%$ \\
\hline C20:3n6 & 117 & 2 & $1.31 \%$ & 176 & 5 & $2.67 \%$ & 5.04 & 0.29 & $5.73 \%$ & 116 & 4 & $3.78 \%$ & 95.4 & 1.2 & $1.31 \%$ & 121 & 0 & $0.29 \%$ \\
\hline C20:4n6 & 799 & 17 & $2.07 \%$ & 1045 & 36 & $3.45 \%$ & 32.1 & 2.0 & $6.10 \%$ & 728 & 35 & $4.81 \%$ & 569 & 3 & $0.60 \%$ & 768 & 7 & $0.92 \%$ \\
\hline $\mathrm{C} 20: 5 \mathrm{n} 3$ & 62.9 & 1.0 & $1.66 \%$ & 87.00 & 1.80 & $2.06 \%$ & $<L O D$ & & & 59.7 & 2.4 & $4.05 \%$ & 50.8 & 0.7 & $1.35 \%$ & 62.2 & 1.0 & $1.65 \%$ \\
\hline C22:0 & 47.0 & 1.1 & $2.37 \%$ & 3.53 & 1.85 & $52.34 \%$ & $<L O D$ & & & 46.7 & 0.6 & $1.25 \%$ & 59.8 & 0.9 & $1.55 \%$ & 61.0 & 0.2 & $0.29 \%$ \\
\hline C22:1n9 & NA & & & & & & $<L O D$ & & & 19.9 & 3.1 & $15.83 \%$ & 2.09 & 0.08 & $3.79 \%$ & 5.03 & 0.51 & $10.20 \%$ \\
\hline C22:4n6 & 17.0 & 0.2 & $1.36 \%$ & 29.4 & 0.3 & $0.96 \%$ & $<L O D$ & & & 32.6 & 3.5 & $10.78 \%$ & 16.1 & 0.2 & $1.17 \%$ & 18.6 & 0.3 & $1.78 \%$ \\
\hline $\mathrm{C} 22: 5 \mathrm{n} 3$ & 33.1 & 0.7 & $2.09 \%$ & 50.6 & 2.7 & $5.35 \%$ & $<L O D$ & & & 31.0 & 1.1 & $3.48 \%$ & 29.3 & 0.6 & $2.05 \%$ & 36.4 & 1.1 & $2.98 \%$ \\
\hline $\mathrm{C} 22: 5 \mathrm{n} 6$ & NA & & & 21.1 & 1.2 & $5.78 \%$ & $<L O D$ & & & 13.8 & 1.7 & $12.02 \%$ & 13.8 & 0.5 & $3.48 \%$ & 16.0 & 0.5 & $3.28 \%$ \\
\hline $\mathrm{C} 22: 6 \mathrm{n} 3$ & 157 & 3 & $1.69 \%$ & 251 & 10 & $3.85 \%$ & 5.89 & 0.35 & $5.89 \%$ & 157 & 8 & $5.19 \%$ & 134 & 0 & $0.35 \%$ & 177 & 2 & $1.27 \%$ \\
\hline $\mathrm{C} 24: 0$ & 39.5 & 0.4 & $1.10 \%$ & 4.10 & 0.76 & $18.51 \%$ & $<L O D$ & & & 41.2 & 2.1 & $5.03 \%$ & 56.5 & 1.8 & $3.13 \%$ & 53.2 & 0.3 & $0.58 \%$ \\
\hline \multirow[t]{5}{*}{$\mathrm{C} 24: 1 \mathrm{n} 9$} & 43.6 & 1.8 & $4.05 \%$ & & & & $<L O D$ & & & 41.9 & 0.8 & $1.90 \%$ & 63.8 & 1.3 & $2.05 \%$ & 63.5 & 1.1 & $1.68 \%$ \\
\hline & & & & & & & & & & \multicolumn{2}{|c|}{ NOTE for Lab 9: } & & & & & & & \\
\hline & & & & & & & & & & C18:1n9 & Oleic acid & & \multicolumn{4}{|c|}{ Total 18:1 determined \& entered } & & \\
\hline & & & & & & & & & & C20:1n9 & \multicolumn{2}{|c|}{ 11-Eicosenoic acid } & \multicolumn{4}{|c|}{ Total 20:1 determined \& entered } & & \\
\hline & & & & & & & & & & C22:1n9 & \multicolumn{2}{|c|}{ Docosenoic acid } & \multicolumn{4}{|c|}{ Total 22:1 determined \& entered } & & \\
\hline
\end{tabular}




\begin{tabular}{|c|c|c|c|c|c|c|c|c|c|c|c|c|c|c|c|c|c|c|}
\hline \multicolumn{19}{|c|}{ Table 5. Interlaboratory data received for SRM 1950} \\
\hline \multirow{3}{*}{ units reported } & $\mu \mathrm{mol} / \mathrm{L}$ & & & $\mu \mathrm{g} / \mathrm{g}$ & & & $\mu \mathrm{mol} / \mathrm{L}$ & & & $\mu \mathrm{mol} / \mathrm{L}$ & & & $\mu \mathrm{mol} / \mathrm{L}$ & & & $\mu \mathrm{g} / \mathrm{g}$ & & \\
\hline & COA & & & Lab 1 & & & Lab 2 & & & Lab 3 & & & Lab 4 & & & Lab 5 & & \\
\hline & Cert/ref & unc & rel unc & mean & stdev & rsd & mean & stdev & rsd & mean & stdev & rsd & mean & stdev & rsd & mean & stdev & rsd \\
\hline C14:0 & 80.1 & 17 & $21.22 \%$ & 26.2 & 0.3 & $1.02 \%$ & 115 & 4 & $3.06 \%$ & 111 & 4 & $3.50 \%$ & 98.7 & 2.2 & $2.25 \%$ & 26.7 & 0.4 & $1.35 \%$ \\
\hline C14:1n5 & 7.1 & 0.1 & $1.41 \%$ & 1.90 & 0.03 & $1.69 \%$ & 16.3 & 0.6 & $3.53 \%$ & NA & & & & & & 1.80 & 0.08 & $4.17 \%$ \\
\hline C16:0 & 2364 & 77 & $3.26 \%$ & 624 & 14 & $2.28 \%$ & 1273 & 25 & $1.98 \%$ & 2210 & 105 & $4.73 \%$ & 2532 & 70 & $2.77 \%$ & 737 & 7 & $0.90 \%$ \\
\hline C16:1n7 & 215 & 26 & $12.09 \%$ & 56.1 & 0.8 & $1.43 \%$ & 252 & 2 & $0.69 \%$ & 271 & 13 & $4.98 \%$ & 271 & 2 & $0.58 \%$ & 84.8 & 1.0 & $1.12 \%$ \\
\hline C18:0 & 644 & 41 & $6.37 \%$ & 174 & 8 & $4.36 \%$ & 612 & 16 & $2.59 \%$ & 652 & 45 & $6.91 \%$ & 707 & 23 & $3.20 \%$ & 237 & 2 & $0.88 \%$ \\
\hline C18:1n7 & 136 & 3 & $2.21 \%$ & 35.6 & 0.9 & $2.39 \%$ & 295 & 4 & $1.22 \%$ & 131 & 7 & $5.05 \%$ & NA & & & 64.8 & 0.6 & $0.94 \%$ \\
\hline C18:1n9 & 1614 & 154 & $9.54 \%$ & 451 & 12 & $2.59 \%$ & 1113 & 21 & $1.87 \%$ & 1717 & 63 & $3.70 \%$ & 1868 & 40 & $2.13 \%$ & 643 & 5 & $0.80 \%$ \\
\hline C18:2n6 & 2838 & 143 & $5.04 \%$ & 879 & 9 & $1.08 \%$ & 3030 & 98 & $3.25 \%$ & 2806 & 104 & $3.69 \%$ & 2607 & 110 & $4.22 \%$ & 991 & 10 & $0.98 \%$ \\
\hline C18:3n3 & 54.6 & 3.6 & $6.59 \%$ & 16.8 & 0.4 & $2.63 \%$ & 52.7 & 0.6 & $1.10 \%$ & 44.7 & 1.2 & $2.68 \%$ & 43.2 & 0.9 & $2.07 \%$ & 17.2 & 0.3 & $1.86 \%$ \\
\hline C18:3n6 & 39.9 & 8.5 & $21.30 \%$ & 13.4 & 0.1 & $0.97 \%$ & 55.3 & 1.5 & $2.76 \%$ & 46.5 & 2.6 & $5.49 \%$ & 47.5 & 2.8 & $5.93 \%$ & 18.1 & 0.1 & $0.32 \%$ \\
\hline C20:0 & 18.0 & 0.5 & $2.78 \%$ & 5.57 & 0.05 & $0.81 \%$ & 51.7 & 1.2 & $2.23 \%$ & 21.1 & 4.2 & $19.70 \%$ & 11.5 & 1.2 & $10.42 \%$ & 4.44 & 0.50 & $11.26 \%$ \\
\hline C20:1n9 & 11.5 & 0.5 & $4.35 \%$ & 3.44 & 0.19 & $5.57 \%$ & NA & & & 15.5 & 4.4 & $28.53 \%$ & 10.3 & 0.9 & $8.85 \%$ & 4.14 & 0.21 & $5.07 \%$ \\
\hline $\mathrm{C} 20: 2 \mathrm{n} 6$ & 18.8 & 0.6 & $3.19 \%$ & NA & & & NA & & & 19.1 & 1.3 & $6.65 \%$ & 18.8 & 1.1 & $6.09 \%$ & 6.82 & 0.07 & $1.03 \%$ \\
\hline C20:3n6 & 139 & 4 & $2.88 \%$ & NA & & & 68.0 & 2.0 & $2.94 \%$ & 141 & 4 & $2.78 \%$ & 124 & 2 & $1.49 \%$ & 50.3 & 0.7 & $1.33 \%$ \\
\hline C20:4n 6 & 984 & 180 & $18.29 \%$ & 274 & 13 & $4.71 \%$ & 1067 & 15 & $1.43 \%$ & 627 & 23 & $3.74 \%$ & 625 & 10 & $1.59 \%$ & 253 & 3 & $0.99 \%$ \\
\hline $\mathrm{C} 20: 5 \mathrm{n} 3$ & 38.6 & 0.5 & $1.30 \%$ & 12.3 & 0.5 & $4.01 \%$ & 60.7 & 2.1 & $3.43 \%$ & 37.4 & 2.1 & $5.56 \%$ & 53.0 & 6.1 & $11.59 \%$ & 14.3 & 0.3 & $1.82 \%$ \\
\hline C22:0 & 47.8 & 6.0 & $12.55 \%$ & 12.3 & 0.4 & $3.01 \%$ & 37.1 & 0.6 & $1.48 \%$ & 16.6 & 4.7 & $28.07 \%$ & 30.4 & 3.8 & $12.56 \%$ & 11.1 & 1.2 & $10.48 \%$ \\
\hline C22:1n9 & 3.4 & 1.3 & $38.24 \%$ & 1.79 & 0.08 & $4.49 \%$ & 8.00 & 0.00 & $0.00 \%$ & NA & & & NA & & & 2.27 & 0.36 & $15.86 \%$ \\
\hline C22:4n6 & 25.5 & 0.6 & $2.35 \%$ & NA & & & 17.3 & 0.6 & $3.33 \%$ & 25.7 & 3.5 & $13.53 \%$ & 20.7 & 1.3 & $6.20 \%$ & 4.17 & 0.17 & $4.08 \%$ \\
\hline C22:5n3 & 38.5 & 0.7 & $1.82 \%$ & 12.5 & 0.3 & $2.78 \%$ & 32.7 & 1.2 & $3.53 \%$ & 38.7 & 4.4 & $11.35 \%$ & 33.4 & 2.2 & $6.72 \%$ & 15.1 & 0.2 & $0.99 \%$ \\
\hline C22:5n6 & 19.5 & 0.4 & $2.05 \%$ & NA & & & 11.7 & 0.6 & $4.95 \%$ & 16.5 & 2.1 & $12.90 \%$ & 18.4 & 0.9 & $4.82 \%$ & 6.74 & 0.20 & $2.97 \%$ \\
\hline $\mathrm{C} 22: 6 \mathrm{n} 3$ & 118 & 21 & $17.80 \%$ & 38.1 & 0.9 & $2.24 \%$ & 94.3 & 2.9 & $3.06 \%$ & 109 & 5 & $4.58 \%$ & 89.8 & 2.0 & $2.26 \%$ & 37.3 & 0.2 & $0.46 \%$ \\
\hline C24:0 & 46.6 & 2.6 & $5.58 \%$ & 15.7 & 0.3 & $1.63 \%$ & 38.0 & 1.1 & $2.77 \%$ & 10.9 & 4.0 & $36.37 \%$ & 13.0 & 1.5 & $11.21 \%$ & 10.2 & 0.7 & $6.55 \%$ \\
\hline C24:1n9 & 71.3 & 3.2 & $4.49 \%$ & 17.1 & 0.3 & $1.64 \%$ & 51.7 & 3.1 & $5.91 \%$ & 16.0 & 3.4 & $20.96 \%$ & 19.3 & 3.8 & $19.64 \%$ & 14.7 & 1.4 & $9.82 \%$ \\
\hline
\end{tabular}

\begin{tabular}{|c|c|c|c|c|c|c|c|c|c|c|c|c|c|c|c|c|c|c|}
\hline \multicolumn{19}{|c|}{ Table 5 (Continued) Interlaboratory data received for SRM 1950} \\
\hline & & & & & & & & & & & & & & & & & & \\
\hline \multirow[t]{3}{*}{ units reported } & $\mu \mathrm{mol} / \mathrm{L}$ & & & $\mu \mathrm{g} / \mathrm{g}$ & & & $\mu \mathrm{mol} / \mathrm{L}$ & & & $\mu \mathrm{g} / \mathrm{g}$ & & & $\mu \mathrm{mol} / \mathrm{L}$ & & & $\mu \mathrm{mol} / \mathrm{L}$ & & \\
\hline & Lab 6 & & & Lab 7 & & & Lab 8 & & & Lab 9 & & & Lab 10 & & & Lab 11 & & \\
\hline & mean & stdev & rsd & mean & stdev & rsd & mean & stdev & rsd & mean & stdev & rsd & mean & stdev & rsd & mean & stdev & rsd \\
\hline C14:0 & 103 & 1 & $0.97 \%$ & 37.3 & 3.9 & $10.45 \%$ & 11.9 & 0.5 & $4.56 \%$ & 25.0 & 0.3 & $1.06 \%$ & 61.4 & 5.0 & $8.14 \%$ & 112 & 3 & $2.36 \%$ \\
\hline C14:1n5 & NA & & & 3.77 & 0.06 & $1.64 \%$ & $<\angle O D$ & & & 2.22 & 0.12 & $5.37 \%$ & 4.31 & 0.66 & $15.24 \%$ & 7.46 & 0.14 & $1.85 \%$ \\
\hline C16:0 & 2343 & 58 & $2.46 \%$ & 793 & 58 & $7.31 \%$ & 341 & 6 & $1.87 \%$ & 612 & 8 & $1.33 \%$ & 1643 & 53 & $3.22 \%$ & 2571 & 71 & $2.76 \%$ \\
\hline C16:1n7 & 272 & 3 & $1.10 \%$ & 15.9 & 0.7 & $4.26 \%$ & 12.4 & 2.0 & $16.47 \%$ & 75.2 & 1.9 & $2.58 \%$ & 184 & 10 & $5.26 \%$ & 277 & 6 & $2.17 \%$ \\
\hline C18:0 & 679 & 8 & $1.11 \%$ & 268 & 16 & $5.90 \%$ & 113 & 4 & $3.31 \%$ & 205 & 1 & $0.27 \%$ & 526 & 6 & $1.21 \%$ & 609 & 19 & $3.15 \%$ \\
\hline C18:1n7 & 133 & 4 & $3.12 \%$ & 54.0 & 14.0 & $25.86 \%$ & 45.4 & 1.7 & $3.76 \%$ & ND & & & 134 & 10 & $7.62 \%$ & 131 & 3 & $2.61 \%$ \\
\hline C18:1n9 & 1837 & 47 & $2.57 \%$ & 718 & 65 & $9.03 \%$ & 116 & 5 & $4.50 \%$ & 608 & 3 & $0.46 \%$ & 1363 & 80 & $5.90 \%$ & 1906 & 38 & $2.01 \%$ \\
\hline C18:2n6 & 2897 & 110 & $3.79 \%$ & 1071 & 112 & $10.46 \%$ & 134 & 5 & $3.96 \%$ & 822 & 0 & $0.02 \%$ & 2081 & 112 & $5.39 \%$ & 3066 & 66 & $2.15 \%$ \\
\hline C18:3n3 & 53.3 & 1.8 & $3.31 \%$ & 21.5 & 1.9 & $8.99 \%$ & $<\angle O D$ & & & 15.6 & 0.1 & $0.82 \%$ & 36.6 & 2.1 & $5.84 \%$ & 57.6 & 1.0 & $1.67 \%$ \\
\hline C18:3n6 & 52.5 & 1.8 & $3.37 \%$ & 20.5 & 2.4 & $11.44 \%$ & 4.48 & 0.52 & $11.62 \%$ & 15.0 & 0.7 & $4.96 \%$ & 36.6 & 2.8 & $7.66 \%$ & 54.3 & 0.7 & $1.36 \%$ \\
\hline C20:0 & NA & & & 11.7 & 0.7 & $6.11 \%$ & $<\angle O D$ & & & 7.97 & 0.45 & $5.61 \%$ & 16.1 & 0.2 & $1.41 \%$ & 18.1 & 0.5 & $2.74 \%$ \\
\hline C20:1n9 & NA & & & 8.62 & 4.32 & $50.13 \%$ & $<\angle O D$ & & & 4.02 & 0.38 & $9.54 \%$ & 10.5 & 1.0 & $9.56 \%$ & 12.4 & 0.2 & $1.29 \%$ \\
\hline$C 20: 2 n 6$ & NA & & & 2.43 & 0.27 & $11.00 \%$ & $<\angle O Q$ & & & 6.15 & 0.34 & $5.53 \%$ & 15.9 & 0.2 & $1.04 \%$ & 19.4 & 0.3 & $1.66 \%$ \\
\hline C20:3n6 & 139 & 2 & $1.25 \%$ & 63.2 & 3.7 & $5.84 \%$ & 12.0 & 0.7 & $6.14 \%$ & 41.9 & 0.3 & $0.83 \%$ & 111 & 1 & $1.27 \%$ & 146 & 2 & $1.45 \%$ \\
\hline C20:4n 6 & 704 & 14 & $2.04 \%$ & 314 & 24 & $7.59 \%$ & 57.4 & 1.0 & $1.68 \%$ & 212 & 2 & $0.71 \%$ & 540 & 16 & $2.97 \%$ & 752 & 18 & $2.37 \%$ \\
\hline C20:5n3 & 42.0 & 1.3 & $3.10 \%$ & 18.9 & 1.8 & $9.59 \%$ & $<L O D$ & & & 12.3 & 0.5 & $4.34 \%$ & 35.8 & 1.4 & $3.90 \%$ & 42.2 & 2.0 & $4.72 \%$ \\
\hline C22:0 & 37.5 & 0.6 & $1.61 \%$ & 1.02 & 0.14 & $13.86 \%$ & $<\angle O D$ & & & 13.4 & 1.1 & $8.31 \%$ & 49.1 & 0.5 & $1.10 \%$ & 52.1 & 1.2 & $2.32 \%$ \\
\hline C22:1n9 & NA & & & & & & $<\angle O D$ & & & 7.01 & 0.81 & $11.49 \%$ & 2.12 & 0.11 & $5.03 \%$ & 4.75 & 0.47 & $9.81 \%$ \\
\hline C22:4n 6 & 24.1 & 0.5 & $1.87 \%$ & 13.9 & 1.0 & $7.46 \%$ & 1.67 & 0.06 & $3.88 \%$ & 13.0 & 0.6 & $4.39 \%$ & 22.4 & 0.6 & $2.50 \%$ & 28.1 & 1.1 & $4.07 \%$ \\
\hline C22: $5 \mathrm{n} 3$ & 38.3 & 0.8 & $1.96 \%$ & 19.9 & 0.8 & $4.08 \%$ & 2.97 & 0.27 & $8.94 \%$ & 12.7 & 0.1 & $0.55 \%$ & 34.6 & 0.2 & $0.68 \%$ & 42.2 & 1.5 & $3.54 \%$ \\
\hline C22:5n6 & NA & & & 9.14 & 0.44 & $4.81 \%$ & $<\angle O D$ & & & 5.73 & 0.60 & $10.45 \%$ & 18.0 & 1.3 & $7.21 \%$ & 21.5 & 0.4 & $1.73 \%$ \\
\hline C22:6n 3 & 95.8 & 2.9 & $3.02 \%$ & 56.4 & 4.6 & $8.14 \%$ & 8.12 & 0.66 & $8.17 \%$ & 32.8 & 1.3 & $4.00 \%$ & 82.3 & 0.5 & $0.57 \%$ & 113 & 3 & $2.66 \%$ \\
\hline C24:0 & 32.1 & 0.7 & $2.08 \%$ & 0.921 & 0.168 & $18.24 \%$ & $<L O D$ & & & 12.1 & 1.3 & $10.38 \%$ & 47.3 & 0.2 & $0.48 \%$ & 45.5 & 1.0 & $2.25 \%$ \\
\hline \multirow[t]{5}{*}{ C24:1n9 } & 48.2 & 1.2 & $2.45 \%$ & & & & $<\angle O D$ & & & 16.4 & 1.2 & $7.44 \%$ & 72.1 & 3.1 & $4.32 \%$ & 71.4 & 1.9 & $2.62 \%$ \\
\hline & & & & & & & & & & \multicolumn{2}{|c|}{ NOTE for Lab 9: } & & & & & & & \\
\hline & & & & & & & & & & C18:1n9 & & & \multicolumn{3}{|c|}{ Total 18:1 determined \& entered } & & & \\
\hline & & & & & & & & & & $C 20: \ln 9$ & \multirow{2}{*}{\multicolumn{2}{|c|}{ 11-Eicosenoic acid }} & \multirow{2}{*}{\multicolumn{3}{|c|}{$\begin{array}{l}\text { Total 20:1 determined \& entered } \\
\text { Total 22:1 determined \& entered }\end{array}$}} & & & \\
\hline & & & & & & & & & & $C 22: \ln 9$ & & & & & & & & \\
\hline
\end{tabular}




\begin{tabular}{|c|c|c|c|c|c|c|c|c|c|c|c|c|c|c|c|c|c|c|}
\hline \multicolumn{19}{|c|}{ Table 5 (Continued) Interlaboratory data received for SRM 1950} \\
\hline & & & & Lab 1 & & & Lab 2 & & & Lab 3 & & & Lab 4 & & & Lab 5 & & \\
\hline & & & & mean & stdev & rsd & mean & stdev & rsd & mean & stdev & rsd & mean & stdev & rsd & mean & stdev & rsd \\
\hline density $(\mathrm{g} / \mathrm{mL})$ & & & & 1.02 & 0.00 & 0.00 & not reporte & & & 1.04 & 0.02 & $1.78 \%$ & not reported & & & 1.01 & 0.02 & $2.11 \%$ \\
\hline & & & & & & & & & & & & & & & & & & \\
\hline & \multicolumn{18}{|c|}{ CONVERT ALL TO $\mu \mathrm{mol} / \mathrm{L}$} \\
\hline & & & & & & & & & & & & & & & & & & \\
\hline C14:0 & Cert/ret & unc & rel unc & mean & stdev & rsd & mean & stdev & rsd & mean & stdev & rsd & mean & stdev & rsd & mean & stdev & rsd \\
\hline & 80.1 & 17 & $21.22 \%$ & 117 & 1 & $1.02 \%$ & 115 & 4 & $3.06 \%$ & 111 & 4 & $3.50 \%$ & 98.7 & 2.2 & $2.25 \%$ & 118 & 2 & $1.35 \%$ \\
\hline C14:1n5 & 7.1 & 0.1 & $1.41 \%$ & 8.55 & 0.14 & $1.69 \%$ & 16.3 & 0.6 & $3.53 \%$ & NA & & & & & & 8.04 & 0.34 & $4.17 \%$ \\
\hline C16:0 & 2364 & 77 & $3.26 \%$ & 2483 & 56 & $2.28 \%$ & 1273 & 25 & $1.98 \%$ & 2210 & 105 & $4.73 \%$ & 2532 & 70 & $2.77 \%$ & 2911 & 26 & $0.90 \%$ \\
\hline C16:1n7 & 215 & 26 & $12.09 \%$ & 225 & 3 & $1.43 \%$ & 252 & 2 & $0.69 \%$ & 271 & 13 & $4.98 \%$ & 271 & 2 & $0.58 \%$ & 338 & 4 & $1.12 \%$ \\
\hline C18:0 & 644 & 41 & $6.37 \%$ & 623 & 27 & $4.36 \%$ & 612 & 16 & $2.59 \%$ & 652 & 45 & $6.91 \%$ & 707 & 23 & $3.20 \%$ & 844 & 7 & $0.88 \%$ \\
\hline C18:1n7 & 136 & 3 & $2.21 \%$ & 129 & 3 & $2.39 \%$ & 295 & 4 & $1.22 \%$ & 131 & 7 & $5.05 \%$ & NA & & & 232 & 2 & $0.94 \%$ \\
\hline C18:1n9 & 1614 & 154 & $9.54 \%$ & 1629 & 42 & $2.59 \%$ & 1113 & 21 & $1.87 \%$ & 1717 & 63 & $3.70 \%$ & 1868 & 40 & $2.13 \%$ & 2305 & 18 & $0.80 \%$ \\
\hline $\mathrm{C} 18: 2 \mathrm{n} 6$ & 2838 & 143 & $5.04 \%$ & 3198 & 34 & $1.08 \%$ & 3030 & 98 & $3.25 \%$ & 2806 & 104 & $3.69 \%$ & 2607 & 110 & $4.22 \%$ & 3578 & 35 & $0.98 \%$ \\
\hline C18:3n3 & 54.6 & 3.6 & $6.59 \%$ & 61.5 & 1.6 & $2.63 \%$ & 52.7 & 0.6 & $1.10 \%$ & 44.7 & 1.2 & $2.68 \%$ & 43.2 & 0.9 & $2.07 \%$ & 62.5 & 1.2 & $1.86 \%$ \\
\hline C18:3n6 & 39.9 & 8.5 & $21.30 \%$ & 49.2 & 0.5 & $0.97 \%$ & 55.3 & 1.5 & $2.76 \%$ & 46.5 & 2.6 & $5.49 \%$ & 47.5 & 2.8 & $5.93 \%$ & 65.7 & 0.2 & $0.32 \%$ \\
\hline C20:0 & 18.0 & 0.5 & $2.78 \%$ & 18.2 & 0.1 & $0.81 \%$ & 51.7 & 1.2 & $2.23 \%$ & 21.1 & 4.2 & $19.70 \%$ & 11.5 & 1.2 & $10.42 \%$ & 14.4 & 1.6 & $11.26 \%$ \\
\hline C20:1n9 & 11.5 & 0.5 & $4.35 \%$ & 11.3 & 0.6 & $5.57 \%$ & NA & & & 15.5 & 4.4 & $28.53 \%$ & 10.3 & 0.9 & $8.85 \%$ & 13.5 & 0.7 & $5.07 \%$ \\
\hline C20:2n6 & 18.8 & 0.6 & $3.19 \%$ & NA & & & NA & & & 19.1 & 1.3 & $6.65 \%$ & 18.8 & 1.1 & $6.09 \%$ & 22.4 & 0.2 & $1.03 \%$ \\
\hline C20:3n6 & 139 & 4 & $2.88 \%$ & NA & & & 68.0 & 2.0 & $2.94 \%$ & 141 & 4 & $2.78 \%$ & 124 & 2 & $1.49 \%$ & 166 & 2 & $1.33 \%$ \\
\hline C20:4n6 & 984 & 180 & $18.29 \%$ & 917 & 43 & $4.71 \%$ & 1067 & 15 & $1.43 \%$ & 627 & 23 & $3.74 \%$ & 625 & 10 & $1.59 \%$ & 842 & 8 & $0.99 \%$ \\
\hline $\mathrm{C} 20: 5 \mathrm{n} 3$ & 38.6 & 0.5 & $1.30 \%$ & 41.5 & 1.7 & $4.01 \%$ & 60.7 & 2.1 & $3.43 \%$ & 37.4 & 2.1 & $5.56 \%$ & 53.0 & 6.1 & $11.59 \%$ & 47.9 & 0.9 & $1.82 \%$ \\
\hline C22:0 & 47.8 & 6.0 & $12.55 \%$ & 36.7 & 1.1 & $3.01 \%$ & 37.1 & 0.6 & $1.48 \%$ & 16.6 & 4.7 & $28.07 \%$ & 30.4 & 3.8 & $12.56 \%$ & 32.9 & 3.5 & $10.48 \%$ \\
\hline C22:1n9 & 3.4 & 1.3 & $38.24 \%$ & 5.39 & 0.24 & $4.49 \%$ & 8.00 & 0.00 & $0.00 \%$ & NA & & & NA & & & 6.79 & 1.08 & $15.86 \%$ \\
\hline C22:4n6 & 25.5 & 0.6 & $2.35 \%$ & NA & & & 17.3 & 0.6 & $3.33 \%$ & 25.7 & 3.5 & $13.53 \%$ & 20.7 & 1.3 & $6.20 \%$ & 12.7 & 0.5 & $4.08 \%$ \\
\hline C22:5n3 & 38.5 & 0.7 & $1.82 \%$ & 38.5 & 1.1 & $2.78 \%$ & 32.7 & 1.2 & $3.53 \%$ & 38.7 & 4.4 & $11.35 \%$ & 33.4 & 2.2 & $6.72 \%$ & 46.3 & 0.5 & $0.99 \%$ \\
\hline C22:5n6 & 19.5 & 0.4 & $2.05 \%$ & NA & & & 11.7 & 0.6 & $4.95 \%$ & 16.5 & 2.1 & $12.90 \%$ & 18.4 & 0.9 & $4.82 \%$ & 20.7 & 0.6 & $2.97 \%$ \\
\hline C22:6n3 & 118 & 21 & $17.80 \%$ & 118 & 3 & $2.24 \%$ & 94.3 & 2.9 & $3.06 \%$ & 109 & 5 & $4.58 \%$ & 89.8 & 2.0 & $2.26 \%$ & 115 & 1 & $0.46 \%$ \\
\hline C24:0 & 46.6 & 2.6 & $5.58 \%$ & 43.6 & 0.7 & $1.63 \%$ & 38.0 & 1.1 & $2.77 \%$ & 10.9 & 4.0 & $36.37 \%$ & 13.0 & 1.5 & $11.21 \%$ & 27.9 & 1.8 & $6.55 \%$ \\
\hline C24:1n9 & 71.3 & 3.2 & $4.49 \%$ & 47.7 & 0.8 & $1.64 \%$ & 51.7 & 3.1 & $5.91 \%$ & 16.0 & 3.4 & $20.96 \%$ & 19.3 & 3.8 & $19.64 \%$ & 40.5 & 4.0 & $9.82 \%$ \\
\hline
\end{tabular}

\begin{tabular}{|c|c|c|c|c|c|c|c|c|c|c|c|c|c|c|c|c|c|c|}
\hline \multicolumn{19}{|c|}{ Table 5 (Continued) Interlaboratory data received for SRM 1950} \\
\hline & Lab 6 & & & Lab 7 & & & Lab 8 & & & Lab 9 & & & Lab 10 & & & Lab 11 & & \\
\hline & mean & stdev & rsd & mean & stdev & rsd & mean & stdev & rsd & mean & stdev & rsd & mean & stdev & rsd & mean & stdev & rsd \\
\hline density $(\mathrm{g} / \mathrm{mL})$ & 1.04 & 0.00 & $0.21 \%$ & 0.989 & 0.016 & $1.59 \%$ & 0.997 & 0.000 & $0.01 \%$ & \multicolumn{2}{|c|}{ not reported use 1} & & & & & 0.973 & 0.008 & $0.86 \%$ \\
\hline \multicolumn{19}{|c|}{ CONVERT ALL TO $\mu \mathrm{mol} / \mathrm{L}$} \\
\hline & Lab 6 & & & Lab 7 & & & Lab 8 & & & Lab 9 & & & Lab 10 & & & Lab 11 & & \\
\hline & mean & stdev & rsd & mean & stdev & rsd & mean & stdev & rsd & mean & stdev & rsd & mean & stdev & rsd & mean & stdev & rsd \\
\hline C14:0 & 103 & 1 & $0.97 \%$ & 162 & 17 & $10.45 \%$ & 11.9 & 0.5 & $4.56 \%$ & 109 & 1 & $1.06 \%$ & 61.4 & 5.0 & $8.14 \%$ & 112 & 3 & $2.36 \%$ \\
\hline C14:1n5 & NA & & & 16.5 & 0.3 & $1.64 \%$ & $<\angle O D$ & & & 9.8 & 0.5 & $5.37 \%$ & 4.31 & 0.66 & $15.24 \%$ & 7.46 & 0.14 & $1.85 \%$ \\
\hline C16:0 & 2343 & 58 & $2.46 \%$ & 3058 & 224 & $7.31 \%$ & 341 & 6 & $1.87 \%$ & 2388 & 32 & $1.33 \%$ & 1643 & 53 & $3.22 \%$ & 2571 & 71 & $2.76 \%$ \\
\hline C16:1n7 & 272 & 3 & $1.10 \%$ & 61.6 & 2.6 & $4.26 \%$ & 12.4 & 2.0 & $16.47 \%$ & 296 & 8 & $2.58 \%$ & 184 & 10 & $5.26 \%$ & 277 & 6 & $2.17 \%$ \\
\hline C18:0 & 679 & 8 & $1.11 \%$ & 932 & 55 & $5.90 \%$ & 113 & 4 & $3.31 \%$ & 722 & 2 & $0.27 \%$ & 526 & 6 & $1.21 \%$ & 609 & 19 & $3.15 \%$ \\
\hline C18:1n7 & 133 & 4 & $3.12 \%$ & 189 & 49 & $25.86 \%$ & 45.4 & 1.7 & $3.76 \%$ & NA & & & 134 & 10 & $7.62 \%$ & 131 & 3 & $2.61 \%$ \\
\hline C18:1n9 & 1837 & 47 & $2.57 \%$ & 2513 & 227 & $9.03 \%$ & 116 & 5 & $4.50 \%$ & 2152 & 10 & $0.46 \%$ & 1363 & 80 & $5.90 \%$ & 1906 & 38 & $2.01 \%$ \\
\hline C18:2n6 & 2897 & 110 & $3.79 \%$ & 3776 & 395 & $10.46 \%$ & 134 & 5 & $3.96 \%$ & 2932 & 1 & $0.02 \%$ & 2081 & 112 & $5.39 \%$ & 3066 & 66 & $2.15 \%$ \\
\hline C18:3n3 & 53.3 & 1.8 & $3.31 \%$ & 76.2 & 6.9 & $8.99 \%$ & $<\angle O D$ & & & 56.2 & 0.5 & $0.82 \%$ & 36.6 & 2.1 & $5.84 \%$ & 57.6 & 1.0 & $1.67 \%$ \\
\hline C18:3n6 & 52.5 & 1.8 & $3.37 \%$ & 72.9 & 8.3 & $11.44 \%$ & 4.48 & 0.52 & $11.62 \%$ & 53.9 & 2.7 & $4.96 \%$ & 36.6 & 2.8 & $7.66 \%$ & 54.3 & 0.7 & $1.36 \%$ \\
\hline C20:0 & NA & & & 36.9 & 2.3 & $6.11 \%$ & $<\angle O D$ & & & 25.5 & 1.4 & $5.61 \%$ & 16.1 & 0.2 & $1.41 \%$ & 18.1 & 0.5 & $2.74 \%$ \\
\hline C20:1n9 & NA & & & 27.4 & 13.8 & $50.13 \%$ & $<\angle O D$ & & & 12.9 & 1.2 & $9.54 \%$ & 10.5 & 1.0 & $9.56 \%$ & 12.4 & 0.2 & $1.29 \%$ \\
\hline $\mathrm{C} 20: 2 \mathrm{n} 6$ & NA & & & 7.78 & 0.86 & $11.00 \%$ & $<\angle O Q$ & & & 19.9 & 1.1 & $5.53 \%$ & 15.9 & 0.2 & $1.04 \%$ & 19.4 & 0.3 & $1.66 \%$ \\
\hline C20:3n 6 & 139 & 2 & $1.25 \%$ & 204 & 12 & $5.84 \%$ & 12.0 & 0.7 & $6.14 \%$ & 137 & 1 & $0.83 \%$ & 111 & 1 & $1.27 \%$ & 146 & 2 & $1.45 \%$ \\
\hline C20:4n 6 & 704 & 14 & $2.04 \%$ & 1019 & 77 & $7.59 \%$ & 57.4 & 1.0 & $1.68 \%$ & 696 & 5 & $0.71 \%$ & 540 & 16 & $2.97 \%$ & 752 & 18 & $2.37 \%$ \\
\hline $\mathrm{C} 20: 5 \mathrm{n} 3$ & 42.0 & 1.3 & $3.10 \%$ & 61.7 & 5.9 & $9.59 \%$ & $<L O D$ & & & 40.6 & 1.8 & $4.34 \%$ & 35.8 & 1.4 & $3.90 \%$ & 42.2 & 2.0 & $4.72 \%$ \\
\hline C22:0 & 37.5 & 0.6 & $1.61 \%$ & 2.95 & 0.41 & $13.86 \%$ & $<L O D$ & & & 39.4 & 3.3 & $8.31 \%$ & 49.1 & 0.5 & $1.10 \%$ & 52.1 & 1.2 & $2.32 \%$ \\
\hline C22:1n9 & NA & & & & & & $<\angle O D$ & & & 20.7 & 2.4 & $11.49 \%$ & 2.12 & 0.11 & $5.03 \%$ & 4.75 & 0.47 & $9.81 \%$ \\
\hline C22:4n 6 & 24.1 & 0.5 & $1.87 \%$ & 41.2 & 3.1 & $7.46 \%$ & 1.67 & 0.06 & $3.88 \%$ & 38.9 & 1.7 & $4.39 \%$ & 22.4 & 0.6 & $2.50 \%$ & 28.1 & 1.1 & $4.07 \%$ \\
\hline $\mathrm{C} 22: 5 \mathrm{n} 3$ & 38.3 & 0.8 & $1.96 \%$ & 59.5 & 2.4 & $4.08 \%$ & 2.97 & 0.27 & $8.94 \%$ & 38.3 & 0.2 & $0.55 \%$ & 34.6 & 0.2 & $0.68 \%$ & 42.2 & 1.5 & $3.54 \%$ \\
\hline C22:5n 6 & NA & & & 27.3 & 1.3 & $4.81 \%$ & $\angle L O D$ & & & 17.3 & 1.8 & $10.45 \%$ & 18.0 & 1.3 & $7.21 \%$ & 21.5 & 0.4 & $1.73 \%$ \\
\hline $\mathrm{C} 22: 6 \mathrm{n} 3$ & 95.8 & 2.9 & $3.02 \%$ & 170 & 14 & $8.14 \%$ & 8.12 & 0.66 & $8.17 \%$ & 100 & 4 & $4.00 \%$ & 82.3 & 0.5 & $0.57 \%$ & 113 & 3 & $2.66 \%$ \\
\hline C24:0 & 32.1 & 0.7 & $2.08 \%$ & 2.47 & 0.45 & $18.24 \%$ & $\angle L O D$ & & & 33.0 & 3.4 & $10.38 \%$ & 47.3 & 0.2 & $0.48 \%$ & 45.5 & 1.0 & $2.25 \%$ \\
\hline \multirow[t]{5}{*}{ C24:1n9 } & 48.2 & 1.2 & $2.45 \%$ & & & & $<\angle O D$ & & & 44.7 & 3.3 & $7.44 \%$ & 72.1 & 3.1 & $4.32 \%$ & 71.4 & 1.9 & $2.62 \%$ \\
\hline & & & & & & & & & & \multicolumn{2}{|c|}{ NOTE for Lab 9: } & & & & & & & \\
\hline & & & & & & & & & & C18:1n9 & Oleic acid & & \multicolumn{3}{|c|}{ Total 18:1 determined \& entered } & & & \\
\hline & & & & & & & & & & C20:1n9 & \multicolumn{2}{|c|}{ 11-Eicosenoic acid } & \multirow{2}{*}{\multicolumn{3}{|c|}{ Total 20:1 determined \& entered }} & & & \\
\hline & & & & & & & & & & C22:1n9 & \multicolumn{2}{|c|}{ Docosenoic acid } & & \multicolumn{2}{|c|}{ Total 22:1 determined \& entered } & & & \\
\hline
\end{tabular}




\begin{tabular}{|c|c|c|c|c|c|c|c|c|c|c|c|c|c|c|}
\hline \multicolumn{15}{|c|}{ Table 6. Additional Data ( $\mu \mathrm{mol} / \mathrm{L}$ ) Reported } \\
\hline & & & & & & & & & & & & & & \multirow[b]{3}{*}{ rsd } \\
\hline Lab & & & \multicolumn{3}{|c|}{ Candidate SRM 2378-1 } & \multicolumn{3}{|c|}{ Candidate SRM 2378-2 } & \multicolumn{3}{|c|}{ Candidate SRM 2378-3 } & \multirow{2}{*}{\multicolumn{2}{|c|}{\begin{tabular}{c|c} 
& SRM 1950 \\
mean & stdev
\end{tabular}}} & \\
\hline reporting & fatty acid ( $r$ & omenclature supplied by lab) & mean & stdev & rsd & mean & stdev & rsd & mean & stdev & rsd & & & \\
\hline 11 & C8:0 & Caprylic (octanoic) & 7.72 & 1.30 & $16.85 \%$ & 1.51 & 0.87 & $57.50 \%$ & 1.68 & 1.65 & $98.42 \%$ & 2.80 & 4.53 & $161.54 \%$ \\
\hline 11 & $\mathrm{C} 10: 0$ & Capric (decanoic) & 21.1 & 0.1 & $0.41 \%$ & 1.89 & 0.34 & $17.75 \%$ & 5.27 & 0.37 & $6.96 \%$ & 2.70 & 0.30 & $11.01 \%$ \\
\hline 7 & C12:0 & & 57.4 & 2.4 & $4.15 \%$ & 22.8 & 2.2 & $9.81 \%$ & 52.3 & 1.5 & $2.90 \%$ & 26.9 & 4.0 & $14.76 \%$ \\
\hline 11 & C12:0 & Lauric (dodecanoic) & 27.4 & 0.3 & $1.24 \%$ & 11.3 & 0.4 & $3.76 \%$ & 28.1 & 0.3 & $1.23 \%$ & 9.27 & 0.04 & $0.47 \%$ \\
\hline 7 & C15:0 & & 41.4 & 6.0 & $14.58 \%$ & 36.9 & 7.1 & $19.13 \%$ & 41.1 & 0.8 & $1.98 \%$ & 30.2 & 1.3 & $4.26 \%$ \\
\hline 11 & C15:0 & Pentadecanoic & 21.7 & 0.3 & $1.25 \%$ & 28.5 & 0.3 & $0.94 \%$ & 20.7 & 0.2 & $1.02 \%$ & 16.3 & 0.5 & $3.06 \%$ \\
\hline 7 & iso-C $16: 0$ & & 10.2 & 1.4 & $14.10 \%$ & 7.72 & 1.43 & $18.55 \%$ & 10.3 & 0.8 & $8.22 \%$ & 7.52 & 0.67 & $8.93 \%$ \\
\hline 3 & $\mathrm{C} 16: 1 \mathrm{n} 7 \mathrm{t}$ & Palmitelaidic Acid & 12.7 & 1.7 & $13.50 \%$ & 29.3 & 3.6 & $12.22 \%$ & 21.7 & 1.0 & $4.55 \%$ & 23.1 & 3.9 & $16.93 \%$ \\
\hline 4 & C16:1n7t & Palmitelaidic Acid & 152 & 7 & $4.32 \%$ & 159 & 6 & $4.01 \%$ & 148 & 4 & $2.90 \%$ & 158 & 4 & $2.49 \%$ \\
\hline 7 & C16:1n-9 & & 16.4 & 3.4 & $20.63 \%$ & 21.2 & 5.1 & $24.29 \%$ & 17.2 & 1.0 & $5.59 \%$ & 31.2 & 2.1 & $6.59 \%$ \\
\hline 7 & C16:1n-5 & & 285 & 6 & $1.99 \%$ & 234 & 27 & $11.61 \%$ & 244 & 9 & $3.51 \%$ & 382 & 38 & $9.93 \%$ \\
\hline 7 & iso-C17:0 & & 16.8 & 2.5 & $14.92 \%$ & 15.2 & 0.7 & $4.71 \%$ & 16.7 & 1.2 & $7.00 \%$ & 10.6 & 1.5 & $14.53 \%$ \\
\hline 11 & C17:0 & Margaric (heptadecanoic) & 26.9 & 0.7 & $2.68 \%$ & 33.4 & 0.3 & $0.97 \%$ & 26.5 & 0.6 & $2.13 \%$ & 25.0 & 0.4 & $1.54 \%$ \\
\hline 3 & C18:1t & total $18: 1 \mathrm{t}$ & 56.5 & 2.4 & $4.33 \%$ & 94.1 & 0.6 & $0.68 \%$ & 84.3 & 1.7 & $1.98 \%$ & 132 & 8 & $5.79 \%$ \\
\hline 4 & C18:1t & & 58.1 & 4.1 & $7.05 \%$ & 98.3 & 3.7 & $3.72 \%$ & 85.9 & 2.3 & $2.69 \%$ & 123 & 5 & $4.09 \%$ \\
\hline 7 & 9t,11c 18:2 & & 15.0 & 1.2 & $7.76 \%$ & 6.52 & 0.66 & $10.07 \%$ & 6.57 & 0.61 & $9.22 \%$ & 6.48 & 0.43 & $6.59 \%$ \\
\hline 3 & C18:2n6t & total $18: 2 \mathrm{t}$ & 41.1 & 0.1 & $0.35 \%$ & 54.0 & 7.1 & $13.22 \%$ & 46.0 & 1.6 & $3.40 \%$ & 62.0 & 4.4 & $7.11 \%$ \\
\hline 4 & $\mathrm{C} 18 \mathrm{2n} 6 \mathrm{t}$ & & 31.1 & 0.7 & $2.12 \%$ & 37.1 & 0.5 & $1.47 \%$ & 25.9 & 5.1 & $19.72 \%$ & 44.9 & 1.5 & $3.31 \%$ \\
\hline 11 & $\mathrm{C} 18: 4 \mathrm{n} 3$ & Stearidonic & 8.13 & 0.46 & $5.70 \%$ & 4.11 & 0.22 & $5.33 \%$ & 2.83 & 0.07 & $2.44 \%$ & 2.57 & 0.06 & $2.37 \%$ \\
\hline 7 & $C 20: 3 n-9$ & & 24.2 & 1.7 & $7.21 \%$ & 23.9 & 4.9 & $20.34 \%$ & 21.7 & 6.5 & $30.10 \%$ & 25.5 & 2.4 & $9.45 \%$ \\
\hline 11 & C20:3n9 & $5 Z, 8 \mathrm{Z}, 11 \mathrm{Z}$-Eicosatrienoic & 4.67 & 0.12 & $2.49 \%$ & 7.10 & 0.09 & $1.31 \%$ & 7.54 & 0.04 & $0.56 \%$ & 8.66 & 0.29 & $3.32 \%$ \\
\hline 11 & C20:3n6 & homo-gamma-Linolenic & 102 & 1 & $1.37 \%$ & 196 & 7 & $3.35 \%$ & 121 & 0 & $0.29 \%$ & 146 & 2 & $1.45 \%$ \\
\hline 7 & $C 224 n-3$ & & 17.2 & 0.7 & $3.84 \%$ & 13.4 & 3.6 & $26.94 \%$ & 10.7 & 0.2 & $2.19 \%$ & 10.8 & 0.3 & $2.49 \%$ \\
\hline 11 & $\mathrm{C} 23: 0$ & Tricosanoic & 23.6 & 0.2 & $0.91 \%$ & 37.9 & 0.6 & $1.57 \%$ & 22.3 & 0.1 & $0.34 \%$ & 21.2 & 0.4 & $1.92 \%$ \\
\hline 11 & C26:0 & Hexacosanoic (cerotic) & 0.891 & 0.049 & $5.54 \%$ & 0.632 & 0.045 & $7.12 \%$ & 0.863 & 0.089 & $10.33 \%$ & 0.549 & 0.073 & $13.25 \%$ \\
\hline
\end{tabular}


Table 7. Consensus Medians ( $\mu \mathrm{mol} / \mathrm{L}$ ) (excluding data from Lab 8) for each sample

\begin{tabular}{|l|c|c|c|c|c|c|c|c|c|}
\hline & \multicolumn{2}{|c|}{ SRM } & \multicolumn{2}{|c|}{ Fatty acid } & \multicolumn{2}{|c|}{ SRM $2378-2$} & \multicolumn{2}{|c|}{ SRM $2378-3$} & \multicolumn{2}{|c|}{ SRM 1950} \\
\hline C14:0 & median & $\mathrm{n}$ & median & $\mathrm{n}$ & median & $\mathrm{n}$ & median & $\mathrm{n}$ \\
\hline C14:1n5 & 193 & 10 & 152 & 10 & 157 & 10 & 112 & 10 \\
\hline C16:0 & 14.0 & 7 & 12.1 & 7 & 12.7 & 7 & 8.55 & 7 \\
\hline C16:1n7 & 2899 & 10 & 2841 & 10 & 2503 & 10 & 2435 & 10 \\
\hline C18:0 & 749 & 10 & 263 & 10 & 176 & 10 & 271 & 10 \\
\hline C18:1n7 & 141 & 8 & 135 & 8 & 120 & 8 & 134 & 8 \\
\hline C18:1n9 & 1910 & 10 & 2586 & 10 & 1932 & 10 & 1852 & 10 \\
\hline C18:2n6 & 3395 & 10 & 4354 & 10 & 3327 & 10 & 2981 & 10 \\
\hline C18:3n3 & 104 & 10 & 110 & 10 & 61.6 & 10 & 54.7 & 10 \\
\hline C18:3n6 & 40.5 & 10 & 74.5 & 10 & 50.8 & 10 & 53.2 & 10 \\
\hline C20:0 & 24.9 & 9 & 26.1 & 9 & 27.6 & 9 & 18.2 & 9 \\
\hline C20:1n9 & 16.3 & 8 & 16.1 & 8 & 17.4 & 8 & 12.7 & 8 \\
\hline C20:2n6 & 17.4 & 7 & 23.1 & 7 & 19.6 & 7 & 19.1 & 7 \\
\hline C20:3n6 & 101 & 9 & 190 & 9 & 117 & 9 & 139 & 9 \\
\hline C20:4n6 & 630 & 10 & 797 & 10 & 784 & 10 & 728 & 10 \\
\hline C20:5n3 & 263 & 10 & 70.2 & 10 & 62.5 & 10 & 42.1 & 10 \\
\hline C22:0 & 42.2 & 10 & 63.2 & 10 & 44.1 & 10 & 36.9 & 10 \\
\hline C22:1n9 & 5.76 & 6 & 7.27 & 6 & 7.27 & 6 & 6.09 & 6 \\
\hline C22:4n6 & 12.4 & 9 & 21.3 & 9 & 17.0 & 9 & 24.1 & 9 \\
\hline C22:5n3 & 65.4 & 10 & 48.8 & 10 & 32.7 & 10 & 38.4 & 10 \\
\hline C22:5n6 & 8.00 & 8 & 14.0 & 8 & 13.8 & 8 & 18.2 & 8 \\
\hline C22:6n3 & 293 & 10 & 171 & 10 & 158 & 10 & 104 & 10 \\
\hline C24:0 & 38.9 & 10 & 50.5 & 10 & 37.1 & 10 & 32.5 & 10 \\
\hline C24:1n9 & 57.3 & 9 & 52.9 & 9 & 41.9 & 9 & 47.7 & 9 \\
\hline
\end{tabular}


Table 8. Percent differences of the Laboratory Means $(n=3)$ from Median of Interlaboratory Data (See Table 7)

\begin{tabular}{|c|c|c|c|c|c|}
\hline Laboaratory \# & Fatty acid & SRM 2378-1 & SRM 2378-2 & SRM 2378-3 & SRM 1950 \\
\hline \multirow[t]{24}{*}{ Lab 1} & C14:0 & $9.59 \%$ & $4.11 \%$ & $-2.91 \%$ & $4.92 \%$ \\
\hline & C14:1n5 & $33.0 \%$ & $25.7 \%$ & $43.0 \%$ & $0.00 \%$ \\
\hline & C16:0 & $26.3 \%$ & $1.04 \%$ & $23.3 \%$ & $1.95 \%$ \\
\hline & C16:1n7 & $6.02 \%$ & $9.53 \%$ & $12.6 \%$ & $-16.9 \%$ \\
\hline & C18:0 & $15.2 \%$ & $1.27 \%$ & $0.19 \%$ & $-6.35 \%$ \\
\hline & C18:1n7 & $20.1 \%$ & $-15.5 \%$ & $9.49 \%$ & $-3.89 \%$ \\
\hline & C18:1n9 & $4.18 \%$ & $-0.97 \%$ & $13.0 \%$ & $-12.1 \%$ \\
\hline & C18:2n6 & $18.0 \%$ & $0.02 \%$ & $1.97 \%$ & $7.28 \%$ \\
\hline & C18:3n3 & $24.0 \%$ & $3.58 \%$ & $0.94 \%$ & $12.3 \%$ \\
\hline & C18:3n6 & $20.5 \%$ & $0.37 \%$ & $9.19 \%$ & $-7.48 \%$ \\
\hline & C20:0 & $-12.7 \%$ & $-11.5 \%$ & $19.8 \%$ & $0.00 \%$ \\
\hline & C20:1n9 & $11.1 \%$ & $0.56 \%$ & $3.67 \%$ & $-10.7 \%$ \\
\hline & $C 20: 2 n 6$ & & & & \\
\hline & $C 20: 3 n 6$ & & & & \\
\hline & C20:4n6 & $11.6 \%$ & $-4.94 \%$ & $4.94 \%$ & $26.0 \%$ \\
\hline & $C 20: 5 n 3$ & $20.5 \%$ & $5.42 \%$ & $13.3 \%$ & $-1.34 \%$ \\
\hline & C22:0 & $6.24 \%$ & $0.45 \%$ & $0.71 \%$ & $-0.56 \%$ \\
\hline & C22:1n9 & $31.4 \%$ & $3.75 \%$ & $17.4 \%$ & $-11.5 \%$ \\
\hline & C22:4n6 & & & & \\
\hline & C22:5n3 & $-3.32 \%$ & $12.8 \%$ & $-1.21 \%$ & $0.17 \%$ \\
\hline & $C 22: 5 n 6$ & & & & \\
\hline & $C 22: 6 n 3$ & $15.0 \%$ & $5.53 \%$ & $0.62 \%$ & $13.5 \%$ \\
\hline & C24:0 & $1.94 \%$ & $-11.5 \%$ & $-6.41 \%$ & $34.0 \%$ \\
\hline & C24:1n9 & $3.47 \%$ & $-6.97 \%$ & $-9.58 \%$ & $0.00 \%$ \\
\hline \multirow[t]{24}{*}{ Lab 2} & C14:0 & $1.73 \%$ & $3.52 \%$ & $0.44 \%$ & $2.78 \%$ \\
\hline & C14:1n5 & $55.0 \%$ & $59.3 \%$ & $52.2 \%$ & $91.0 \%$ \\
\hline & C16:0 & $-49.2 \%$ & $-46.4 \%$ & $-47.8 \%$ & $-47.7 \%$ \\
\hline & C16:1n7 & $3.02 \%$ & $0.41 \%$ & $-1.49 \%$ & $-7.00 \%$ \\
\hline & C18:0 & $-7.38 \%$ & $-10.3 \%$ & $-10.8 \%$ & $-8.04 \%$ \\
\hline & C18:1n7 & $66.6 \%$ & $95.8 \%$ & $76.4 \%$ & $121 \%$ \\
\hline & C18:1n9 & $-41.7 \%$ & $-49.6 \%$ & $-44.3 \%$ & $-39.9 \%$ \\
\hline & C18:2n6 & $0.25 \%$ & $-6.59 \%$ & $-0.02 \%$ & $1.64 \%$ \\
\hline & C18:3n3 & $0.32 \%$ & $5.73 \%$ & $-0.94 \%$ & $-3.76 \%$ \\
\hline & C18:3n6 & $3.62 \%$ & $10.0 \%$ & $-0.97 \%$ & $3.96 \%$ \\
\hline & C20:0 & $173 \%$ & $201 \%$ & $113 \%$ & $184 \%$ \\
\hline & C20:1n9 & & & & \\
\hline & $C 20: 2 n 6$ & & & & \\
\hline & $C 20: 3 n 6$ & $-41.3 \%$ & $-52.9 \%$ & $-46.9 \%$ & $-51.1 \%$ \\
\hline & C20:4n6 & $56.7 \%$ & $67.0 \%$ & $43.8 \%$ & $46.6 \%$ \\
\hline & $C 20: 5 n 3$ & $40.6 \%$ & $64.3 \%$ & $39.1 \%$ & $44.2 \%$ \\
\hline & C22:0 & $0.15 \%$ & $-6.31 \%$ & $-4.36 \%$ & $0.56 \%$ \\
\hline & C22:1n9 & $27.2 \%$ & $-3.75 \%$ & $-17.4 \%$ & $31.4 \%$ \\
\hline & $\mathrm{C} 22: 4 \mathrm{n} 6$ & $-27.4 \%$ & $-17.2 \%$ & $-27.6 \%$ & $-28.0 \%$ \\
\hline & $C 22: 5 n 3$ & $-20.5 \%$ & $-7.04 \%$ & $-12.3 \%$ & $-14.9 \%$ \\
\hline & C22:5n6 & $-25.0 \%$ & $-30.9 \%$ & $-37.3 \%$ & $-35.8 \%$ \\
\hline & $C 22: 6 n 3$ & $21.4 \%$ & $7.33 \%$ & $-0.62 \%$ & $-9.49 \%$ \\
\hline & C24:0 & $19.7 \%$ & $19.1 \%$ & $17.0 \%$ & $16.9 \%$ \\
\hline & C24:1n9 & $10.6 \%$ & $27.2 \%$ & $15.3 \%$ & $8.28 \%$ \\
\hline
\end{tabular}


Table 8 (cont). Percent differences of the Laboratory Means ( $n=3$ ) from Median of Interlaboratory Data (See Table 7)

\begin{tabular}{|c|c|c|c|c|c|}
\hline \multirow[t]{2}{*}{ Lab 3} & C14:0 & $-2.50 \%$ & $0.25 \%$ & $3.86 \%$ & $-0.39 \%$ \\
\hline & \multicolumn{5}{|l|}{ C14:1n5 } \\
\hline & C16:0 & $-8.62 \%$ & $-1.04 \%$ & $-4.07 \%$ & $-9.27 \%$ \\
\hline & C16:1n7 & $-7.04 \%$ & $-0.41 \%$ & $1.92 \%$ & $-0.16 \%$ \\
\hline & C18:0 & $-1.66 \%$ & $-1.27 \%$ & $-0.19 \%$ & $-2.03 \%$ \\
\hline & C18:1n7 & $-16.0 \%$ & $-19.5 \%$ & $-23.0 \%$ & $-2.12 \%$ \\
\hline & C18:1n9 & $-7.01 \%$ & $0.97 \%$ & $-4.75 \%$ & $-7.30 \%$ \\
\hline & C18:2n6 & $-8.91 \%$ & $0.56 \%$ & $-1.37 \%$ & $-5.87 \%$ \\
\hline & C18:3n3 & $-14.4 \%$ & $-12.2 \%$ & $-16.2 \%$ & $-18.3 \%$ \\
\hline & C18:3n6 & $-4.53 \%$ & $-4.31 \%$ & $-8.39 \%$ & $-12.6 \%$ \\
\hline & C20:0 & $9.34 \%$ & $10.2 \%$ & $10.7 \%$ & $16.2 \%$ \\
\hline & C20:1n9 & $10.8 \%$ & $28.1 \%$ & $19.1 \%$ & $22.3 \%$ \\
\hline & C20:2n6 & $13.7 \%$ & $5.84 \%$ & $4.63 \%$ & $0.00 \%$ \\
\hline & C20:3n6 & $0.00 \%$ & $5.73 \%$ & $7.58 \%$ & $1.35 \%$ \\
\hline & C20:4n6 & $-16.1 \%$ & $-6.32 \%$ & $-11.8 \%$ & $-13.8 \%$ \\
\hline & C20:5n3 & $-7.37 \%$ & $-8.29 \%$ & $-4.31 \%$ & $-11.1 \%$ \\
\hline & C22:0 & $-62.2 \%$ & $-56.0 \%$ & $-53.2 \%$ & $-55.1 \%$ \\
\hline & C22:1n9 & & & & \\
\hline & C22:4n6 & $-7.09 \%$ & $-1.79 \%$ & $15.7 \%$ & $6.98 \%$ \\
\hline & $C 22: 5 n 3$ & $-0.53 \%$ & $1.02 \%$ & $12.1 \%$ & $0.89 \%$ \\
\hline & $C 22: 5 n 6$ & $-8.47 \%$ & $-14.1 \%$ & $-7.80 \%$ & $-9.23 \%$ \\
\hline & $C 22: 6 n 3$ & $-1.97 \%$ & $-4.60 \%$ & $9.76 \%$ & $4.14 \%$ \\
\hline & C24:0 & $-68.0 \%$ & $-63.6 \%$ & $-55.7 \%$ & $-66.4 \%$ \\
\hline & C24:1n9 & $-68.2 \%$ & $-58.7 \%$ & $-62.2 \%$ & $-66.5 \%$ \\
\hline \multirow[t]{24}{*}{ Lab 4} & C14:0 & $-19.6 \%$ & $-17.5 \%$ & $-17.8 \%$ & $-11.6 \%$ \\
\hline & C14:1n5 & & & & \\
\hline & C16:0 & $-2.67 \%$ & $-2.78 \%$ & $-0.33 \%$ & $3.98 \%$ \\
\hline & C16:1n7 & $-2.85 \%$ & $-7.95 \%$ & $-1.00 \%$ & $0.16 \%$ \\
\hline & C18:0 & $1.66 \%$ & $-3.07 \%$ & $-1.03 \%$ & $6.24 \%$ \\
\hline & C18:1n7 & & & & \\
\hline & C18:1n9 & $-4.18 \%$ & $-4.82 \%$ & $-8.47 \%$ & $0.85 \%$ \\
\hline & C18:2n6 & $-19.4 \%$ & $-17.0 \%$ & $-19.2 \%$ & $-12.6 \%$ \\
\hline & C18:3n3 & $-23.8 \%$ & $-21.3 \%$ & $-33.8 \%$ & $-21.1 \%$ \\
\hline & C18:3n6 & $-6.90 \%$ & $-12.9 \%$ & $-17.5 \%$ & $-10.7 \%$ \\
\hline & C2O:0 & $-36.7 \%$ & $-41.8 \%$ & $-54.8 \%$ & $-37.0 \%$ \\
\hline & C20:1n9 & $-27.5 \%$ & $-23.9 \%$ & $-17.5 \%$ & $-18.5 \%$ \\
\hline & C20:2n6 & $13.0 \%$ & $-24.7 \%$ & $-15.6 \%$ & $-1.56 \%$ \\
\hline & C20:3n6 & $-13.2 \%$ & $-11.8 \%$ & $-12.9 \%$ & $-10.6 \%$ \\
\hline & C20:4n6 & $-21.7 \%$ & $-16.2 \%$ & $-23.9 \%$ & $-14.1 \%$ \\
\hline & C20:5n3 & $-10.3 \%$ & $8.54 \%$ & $-1.81 \%$ & $25.9 \%$ \\
\hline & C22:0 & $-49.2 \%$ & $-46.4 \%$ & $-36.5 \%$ & $-17.7 \%$ \\
\hline & C22:1n9 & & & & \\
\hline & C22:4n6 & $-16.8 \%$ & $-16.4 \%$ & $-23.2 \%$ & $-13.9 \%$ \\
\hline & C22:5n3 & $-20.5 \%$ & $-20.0 \%$ & $-17.5 \%$ & $-13.1 \%$ \\
\hline & C22:5n6 & $0.00 \%$ & $-3.84 \%$ & $-16.9 \%$ & $1.03 \%$ \\
\hline & C22:6n3 & $-17.8 \%$ & $-15.3 \%$ & $-21.6 \%$ & $-13.9 \%$ \\
\hline & C24:0 & $-75.8 \%$ & $-58.0 \%$ & $-56.1 \%$ & $-60.0 \%$ \\
\hline & C24:1n9 & $-62.1 \%$ & $-61.5 \%$ & $-68.8 \%$ & $-59.6 \%$ \\
\hline
\end{tabular}


Table 8 (cont). Percent differences of the Laboratory Means $(n=3)$ from Median of Interlaboratory Data (See Table 7)

\begin{tabular}{|c|c|c|c|c|c|}
\hline \multirow[t]{2}{*}{ Lab 6} & C14:0 & $-1.73 \%$ & $-6.67 \%$ & $-4.86 \%$ & $-7.67 \%$ \\
\hline & \multicolumn{5}{|l|}{ C14:1n5 } \\
\hline & C16:0 & $2.67 \%$ & $4.32 \%$ & $-1.19 \%$ & $-3.78 \%$ \\
\hline & C16:1n7 & $4.51 \%$ & $1.17 \%$ & $2.86 \%$ & $0.38 \%$ \\
\hline & C18:0 & $7.62 \%$ & $3.44 \%$ & $5.09 \%$ & $2.03 \%$ \\
\hline & C18:1n7 & $1.68 \%$ & $8.15 \%$ & $-1.68 \%$ & $-0.30 \%$ \\
\hline & C18:1n9 & $5.59 \%$ & $3.64 \%$ & $-0.29 \%$ & $-0.85 \%$ \\
\hline & C18:2n6 & $-0.25 \%$ & $-0.01 \%$ & $0.48 \%$ & $-2.83 \%$ \\
\hline & C18:3n3 & $-0.32 \%$ & $-1.84 \%$ & $-6.20 \%$ & $-2.67 \%$ \\
\hline & C18:3n6 & $0.58 \%$ & $-0.37 \%$ & $-1.10 \%$ & $-1.36 \%$ \\
\hline & C20:0 & & & & \\
\hline & C20:1n9 & & & & \\
\hline & $\mathrm{C} 20: 2 \mathrm{n} 6$ & & & & \\
\hline & C20:3n6 & $3.18 \%$ & $-0.14 \%$ & $0.00 \%$ & $0.00 \%$ \\
\hline & $C 20: 4 n 6$ & $-0.69 \%$ & $3.28 \%$ & $2.00 \%$ & $-3.32 \%$ \\
\hline & C20:5n3 & $-2.27 \%$ & $-2.44 \%$ & $0.55 \%$ & $-0.28 \%$ \\
\hline & C22:0 & $5.44 \%$ & $10.2 \%$ & $6.60 \%$ & $1.65 \%$ \\
\hline & C22:1n9 & & & & \\
\hline & C22:4n6 & $0.00 \%$ & $0.00 \%$ & $0.00 \%$ & $0.00 \%$ \\
\hline & $C 22: 5 n 3$ & $1.20 \%$ & $-1.02 \%$ & $1.21 \%$ & $-0.19 \%$ \\
\hline & $C 22: 5 n 6$ & & & & \\
\hline & $C 22: 6 n 3$ & $-3.47 \%$ & $-8.64 \%$ & $-0.62 \%$ & $-8.11 \%$ \\
\hline & C24:0 & $8.52 \%$ & $15.4 \%$ & $6.41 \%$ & $-1.36 \%$ \\
\hline & C24:1n9 & $9.05 \%$ & $16.4 \%$ & $4.09 \%$ & $1.01 \%$ \\
\hline \multirow[t]{24}{*}{ Lab 7} & C14:0 & $48.5 \%$ & $0.10 \%$ & $42.5 \%$ & $44.8 \%$ \\
\hline & C14:1n5 & $104 \%$ & $23.8 \%$ & $93.2 \%$ & $92.6 \%$ \\
\hline & C16:0 & $34.0 \%$ & $-2.08 \%$ & $27.3 \%$ & $25.6 \%$ \\
\hline & C16:1n7 & $-72.6 \%$ & $-75.7 \%$ & $-66.8 \%$ & $-77.3 \%$ \\
\hline & C18:0 & $54.1 \%$ & $13.9 \%$ & $43.7 \%$ & $40.0 \%$ \\
\hline & C18:1n7 & $6.73 \%$ & $-12.5 \%$ & $1.68 \%$ & $41.4 \%$ \\
\hline & C18:1n9 & $46.0 \%$ & $-4.35 \%$ & $34.7 \%$ & $35.7 \%$ \\
\hline & C18:2n6 & $24.5 \%$ & $-27.0 \%$ & $19.4 \%$ & $26.7 \%$ \\
\hline & C18:3n3 & $41.2 \%$ & $-2.77 \%$ & $37.4 \%$ & $39.3 \%$ \\
\hline & C18:3n6 & $41.9 \%$ & $-26.9 \%$ & $30.3 \%$ & $37.1 \%$ \\
\hline & C2O:0 & $12.0 \%$ & $-4.23 \%$ & $17.3 \%$ & $103 \%$ \\
\hline & C20:1n9 & $196 \%$ & $131 \%$ & $67.3 \%$ & $117 \%$ \\
\hline & $C 20: 2 n 6$ & $-51.2 \%$ & $-74.1 \%$ & $-66.4 \%$ & $-59.2 \%$ \\
\hline & C20:3n6 & $56.7 \%$ & $10.4 \%$ & $50.9 \%$ & $46.7 \%$ \\
\hline & C20:4n6 & $44.1 \%$ & $-2.04 \%$ & $33.3 \%$ & $40.0 \%$ \\
\hline & C20:5n3 & $39.9 \%$ & $-3.14 \%$ & $39.2 \%$ & $46.6 \%$ \\
\hline & C22:0 & $-88.1 \%$ & $-96.3 \%$ & $-92.0 \%$ & $-92.0 \%$ \\
\hline & C22:1n9 & & & & \\
\hline & $\mathrm{C} 22: 4 \mathrm{n} 6$ & $73.4 \%$ & $28.2 \%$ & $72.8 \%$ & $71.2 \%$ \\
\hline & $C 22: 5 n 3$ & $52.7 \%$ & $9.66 \%$ & $54.9 \%$ & $54.9 \%$ \\
\hline & $C 22: 5 n 6$ & $60.5 \%$ & $19.2 \%$ & $52.7 \%$ & $50.5 \%$ \\
\hline & C22:6n3 & $53.5 \%$ & $13.8 \%$ & $59.0 \%$ & $62.7 \%$ \\
\hline & C24:0 & $-90.3 \%$ & $-94.8 \%$ & $-89.0 \%$ & $-92.4 \%$ \\
\hline & C24:1n9 & & & & \\
\hline
\end{tabular}


Table 8 (cont). Percent differences of the Laboratory Means $(n=3)$ from Median of Interlaboratory Data (See Table 7)

\begin{tabular}{|c|c|c|c|c|c|}
\hline \multirow[t]{24}{*}{ Lab 9} & C14:0 & $1.76 \%$ & $-1.41 \%$ & $-0.02 \%$ & $-1.86 \%$ \\
\hline & C14:1n5 & $10.7 \%$ & $0.00 \%$ & $-2.09 \%$ & $14.8 \%$ \\
\hline & C16:0 & $4.12 \%$ & $5.42 \%$ & $0.33 \%$ & $-1.95 \%$ \\
\hline & C16:1n7 & $3.61 \%$ & $-1.73 \%$ & $-0.40 \%$ & $9.12 \%$ \\
\hline & C18:0 & $12.4 \%$ & $6.11 \%$ & $4.49 \%$ & $8.45 \%$ \\
\hline & C18:1n7 & & & & \\
\hline & C18:1n9 & $17.8 \%$ & $13.2 \%$ & $7.19 \%$ & $16.2 \%$ \\
\hline & C18:2n6 & $0.25 \%$ & $0.01 \%$ & $-2.90 \%$ & $-1.64 \%$ \\
\hline & C18:3n3 & $5.47 \%$ & $1.84 \%$ & $1.71 \%$ & $2.67 \%$ \\
\hline & C18:3n6 & $7.43 \%$ & $2.02 \%$ & $1.73 \%$ & $1.36 \%$ \\
\hline & C20:0 & $10.4 \%$ & $30.3 \%$ & $0.00 \%$ & $40.3 \%$ \\
\hline & C20:1n9 & $-1.76 \%$ & $-11.0 \%$ & $-24.2 \%$ & $2.17 \%$ \\
\hline & $C 20: 2 n 6$ & $12.5 \%$ & $7.33 \%$ & $9.44 \%$ & $4.65 \%$ \\
\hline & $C 20: 3 n 6$ & $5.93 \%$ & $0.00 \%$ & $-0.22 \%$ & $-1.75 \%$ \\
\hline & $C 20: 4 n 6$ & $1.22 \%$ & $2.04 \%$ & $-7.14 \%$ & $-4.31 \%$ \\
\hline & $C 20: 5 n 3$ & $2.27 \%$ & $-3.99 \%$ & $-4.46 \%$ & $-3.63 \%$ \\
\hline & C22:0 & $9.37 \%$ & $5.53 \%$ & $5.93 \%$ & $6.78 \%$ \\
\hline & C22:1n9 & $229 \%$ & $124 \%$ & $174 \%$ & $240 \%$ \\
\hline & $C 22: 4 n 6$ & $141 \%$ & $96.2 \%$ & $91.3 \%$ & $61.8 \%$ \\
\hline & $C 22: 5 n 3$ & $1.08 \%$ & $-4.82 \%$ & $-5.32 \%$ & $-0.17 \%$ \\
\hline & $C 22: 5 n 6$ & $13.9 \%$ & $-4.99 \%$ & $0.09 \%$ & $-4.61 \%$ \\
\hline & $C 22: 6 n 3$ & $1.97 \%$ & $-6.64 \%$ & $-0.66 \%$ & $-4.14 \%$ \\
\hline & C24:0 & $9.31 \%$ & $7.47 \%$ & $11.1 \%$ & $1.36 \%$ \\
\hline & C24:1n9 & $0.00 \%$ & $0.00 \%$ & $0.00 \%$ & $-6.23 \%$ \\
\hline \multirow[t]{24}{*}{ Lab 10} & C14:0 & $-33.6 \%$ & $-43.2 \%$ & $-43.8 \%$ & $-44.9 \%$ \\
\hline & C14:1n5 & $-47.8 \%$ & $-54.8 \%$ & $-55.2 \%$ & $-49.6 \%$ \\
\hline & C16:0 & $-21.0 \%$ & $-27.6 \%$ & $-31.3 \%$ & $-32.5 \%$ \\
\hline & C16:1n7 & $-25.3 \%$ & $-30.6 \%$ & $-31.7 \%$ & $-32.1 \%$ \\
\hline & C18:0 & $-7.04 \%$ & $-20.4 \%$ & $-21.0 \%$ & $-20.9 \%$ \\
\hline & C18:1n7 & $-1.68 \%$ & $-3.71 \%$ & $-10.9 \%$ & $0.30 \%$ \\
\hline & C18:1n9 & $-16.1 \%$ & $-23.3 \%$ & $-28.3 \%$ & $-26.4 \%$ \\
\hline & C18:2n6 & $-22.5 \%$ & $-26.5 \%$ & $-29.9 \%$ & $-30.2 \%$ \\
\hline & C18:3n3 & $-22.5 \%$ & $-28.5 \%$ & $-33.0 \%$ & $-33.1 \%$ \\
\hline & C18:3n6 & $-23.9 \%$ & $-27.0 \%$ & $-33.2 \%$ & $-31.3 \%$ \\
\hline & C2O:0 & $3.85 \%$ & $0.00 \%$ & $-30.7 \%$ & $-11.4 \%$ \\
\hline & C20:1n9 & $-0.31 \%$ & $-24.3 \%$ & $-24.7 \%$ & $-17.2 \%$ \\
\hline & $C 20: 2 n 6$ & $-19.4 \%$ & $-16.2 \%$ & $-18.6 \%$ & $-16.6 \%$ \\
\hline & $C 20: 3 n 6$ & $-6.25 \%$ & $-17.8 \%$ & $-18.3 \%$ & $-20.0 \%$ \\
\hline & $C 20: 4 n 6$ & $-15.3 \%$ & $-18.9 \%$ & $-27.4 \%$ & $-25.8 \%$ \\
\hline & $C 20: 5 n 3$ & $-13.4 \%$ & $-10.8 \%$ & $-18.8 \%$ & $-14.9 \%$ \\
\hline & C22:0 & $58.9 \%$ & $40.1 \%$ & $35.5 \%$ & $32.9 \%$ \\
\hline & C22:1n9 & $-43.7 \%$ & $-69.7 \%$ & $-71.2 \%$ & $-65.2 \%$ \\
\hline & $C 22: 4 n 6$ & $5.90 \%$ & $-5.49 \%$ & $-5.69 \%$ & $-6.80 \%$ \\
\hline & $C 22: 5 n 3$ & $0.53 \%$ & $-9.11 \%$ & $-10.4 \%$ & $-9.83 \%$ \\
\hline & $C 22: 5 n 6$ & $0.00 \%$ & $3.84 \%$ & $-0.09 \%$ & $-1.03 \%$ \\
\hline & $C 22: 6 n 3$ & $-5.75 \%$ & $-20.8 \%$ & $-15.2 \%$ & $-21.0 \%$ \\
\hline & $\mathrm{C} 24: 0$ & $79.9 \%$ & $62.1 \%$ & $52.2 \%$ & $45.5 \%$ \\
\hline & C24:1n9 & $80.7 \%$ & $59.4 \%$ & $52.1 \%$ & $51.1 \%$ \\
\hline
\end{tabular}


Table 8 (cont). Percent differences of the Laboratory Means $(n=3)$ from Median of Interlaboratory Data (See Table 7)

\begin{tabular}{|c|c|c|c|c|c|}
\hline Lab 11 & C14:0 & $2.77 \%$ & $-0.10 \%$ & $0.02 \%$ & $0.39 \%$ \\
\hline & C14:1n5 & $-12.0 \%$ & $-23.9 \%$ & $-18.0 \%$ & $-12.8 \%$ \\
\hline & C16:0 & $7.37 \%$ & $10.9 \%$ & $3.55 \%$ & $5.57 \%$ \\
\hline & C16:1n7 & $2.85 \%$ & $0.53 \%$ & $0.40 \%$ & $2.22 \%$ \\
\hline & C18:0 & $-4.67 \%$ & $-7.65 \%$ & $-11.6 \%$ & $-8.49 \%$ \\
\hline & C18:1n7 & $-3.29 \%$ & $3.71 \%$ & $-8.35 \%$ & $-2.05 \%$ \\
\hline & C18:1n9 & $8.70 \%$ & $6.89 \%$ & $0.29 \%$ & $2.89 \%$ \\
\hline & C18:2n6 & $0.80 \%$ & $2.88 \%$ & $0.02 \%$ & $2.85 \%$ \\
\hline & C18:3n3 & $7.69 \%$ & $7.25 \%$ & $1.57 \%$ & $5.18 \%$ \\
\hline & C18:3n6 & $-0.58 \%$ & $2.36 \%$ & $0.97 \%$ & $1.94 \%$ \\
\hline & C2O:0 & $0.00 \%$ & $12.7 \%$ & $-23.2 \%$ & $-0.23 \%$ \\
\hline & C20:1n9 & $0.31 \%$ & $-0.56 \%$ & $-3.67 \%$ & $-2.17 \%$ \\
\hline & $C 20: 2 n 6$ & $0.00 \%$ & $0.00 \%$ & $0.00 \%$ & $1.80 \%$ \\
\hline & $C 20: 3 n 6$ & $0.87 \%$ & $3.38 \%$ & $3.60 \%$ & $5.04 \%$ \\
\hline & $C 20: 4 n 6$ & $0.69 \%$ & $7.47 \%$ & $-2.00 \%$ & $3.32 \%$ \\
\hline & $C 20: 5 n 3$ & $3.43 \%$ & $2.44 \%$ & $-0.55 \%$ & $0.28 \%$ \\
\hline & C22:0 & $39.1 \%$ & $46.7 \%$ & $38.4 \%$ & $41.1 \%$ \\
\hline & C22:1n9 & $-26.9 \%$ & $-38.7 \%$ & $-30.8 \%$ & $-22.0 \%$ \\
\hline & $C 22: 4 n 6$ & $0.00 \%$ & $8.00 \%$ & $9.02 \%$ & $16.9 \%$ \\
\hline & $C 22: 5 n 3$ & $7.83 \%$ & $6.76 \%$ & $11.4 \%$ & $9.88 \%$ \\
\hline & $C 22: 5 n 6$ & $4.62 \%$ & $15.0 \%$ & $15.5 \%$ & $18.0 \%$ \\
\hline & $C 22: 6 n 3$ & $9.15 \%$ & $4.60 \%$ & $12.2 \%$ & $8.42 \%$ \\
\hline & C24:0 & $44.5 \%$ & $54.4 \%$ & $43.4 \%$ & $39.9 \%$ \\
\hline & C24:1n9 & $52.1 \%$ & $63.2 \%$ & $51.5 \%$ & $49.7 \%$ \\
\hline
\end{tabular}


Appendix A. CHARTS by FATTY ACID and MATERIAL

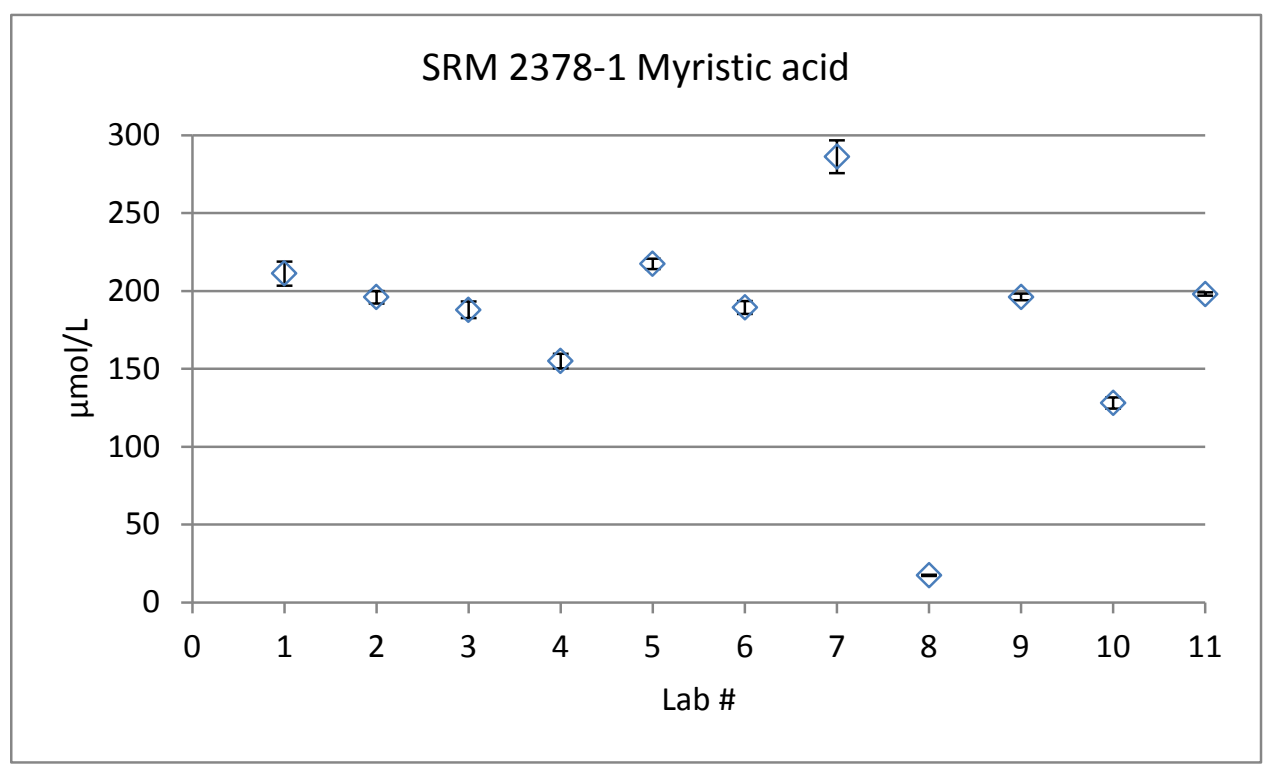

Median (excluding lab 8) $193 \mu \mathrm{mol} / \mathrm{L}$

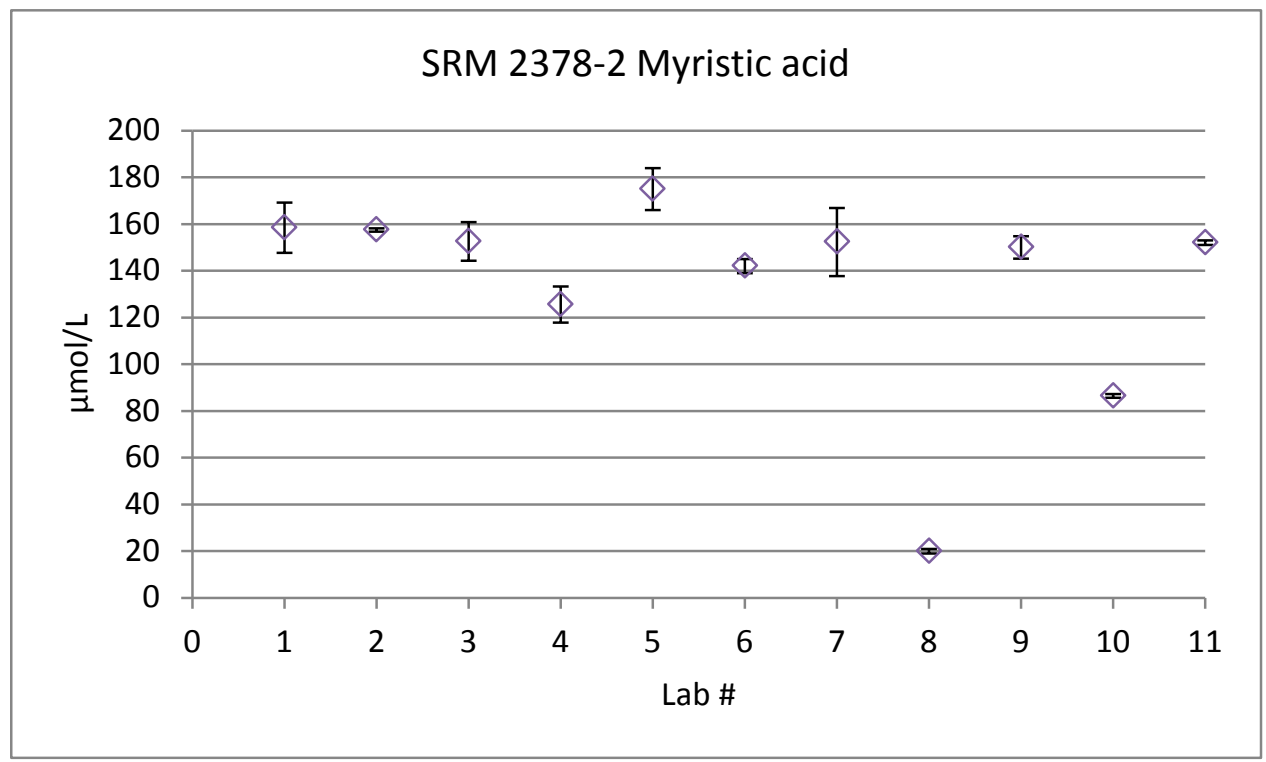

Median (excluding lab 8) $152 \mu \mathrm{mol} / \mathrm{L}$ 

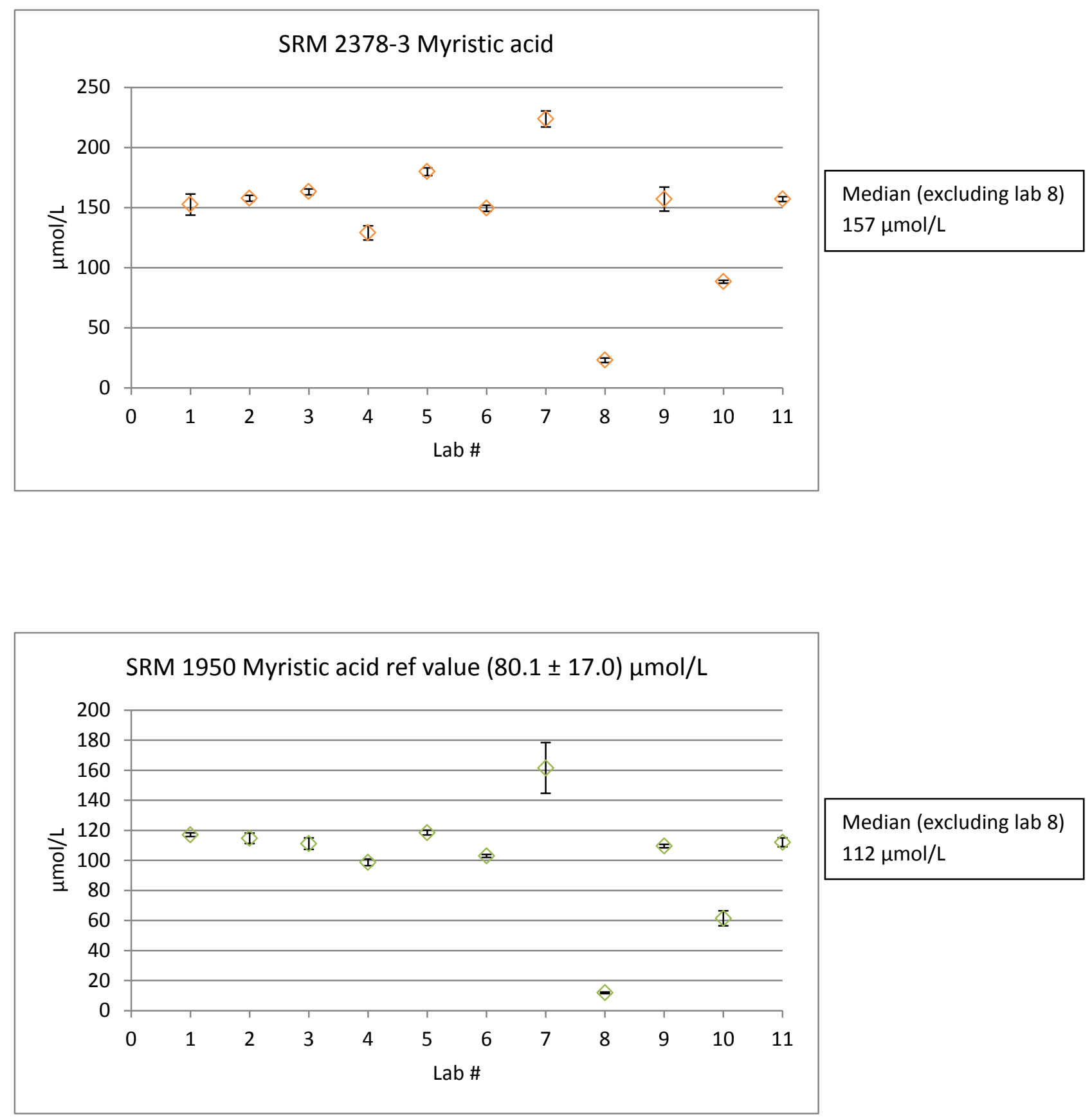


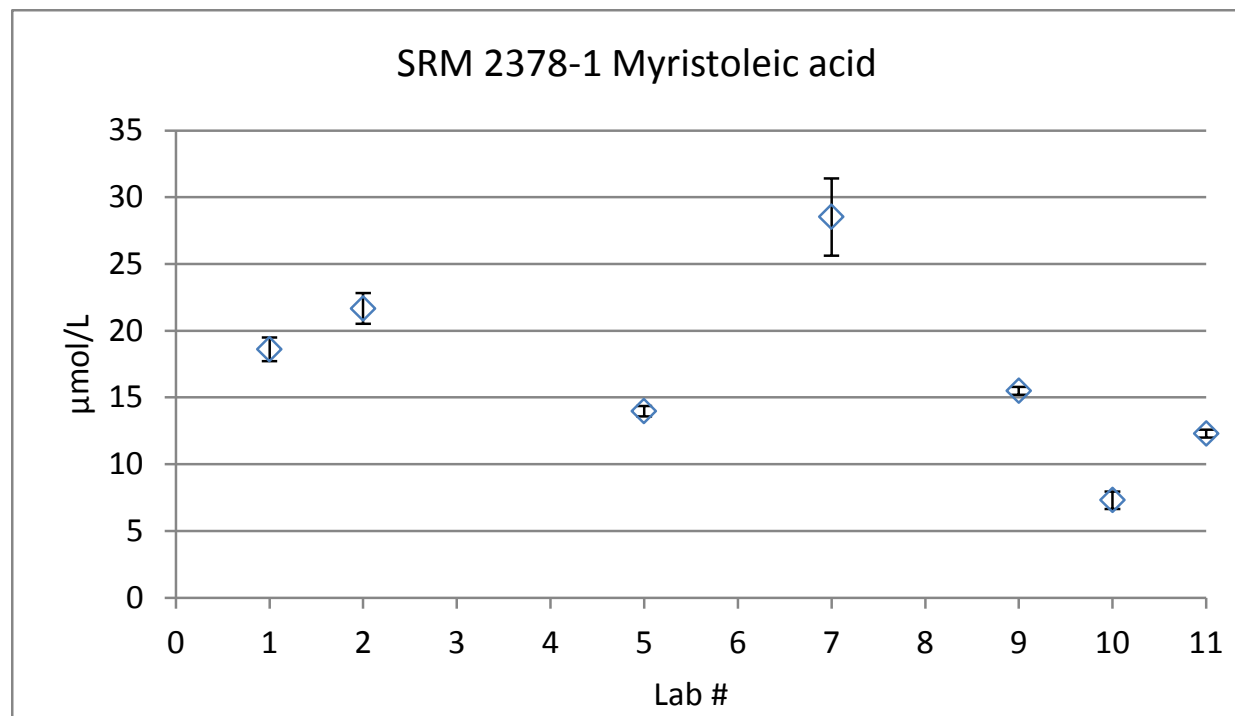

Median $14.0 \mu \mathrm{mol} / \mathrm{L}$

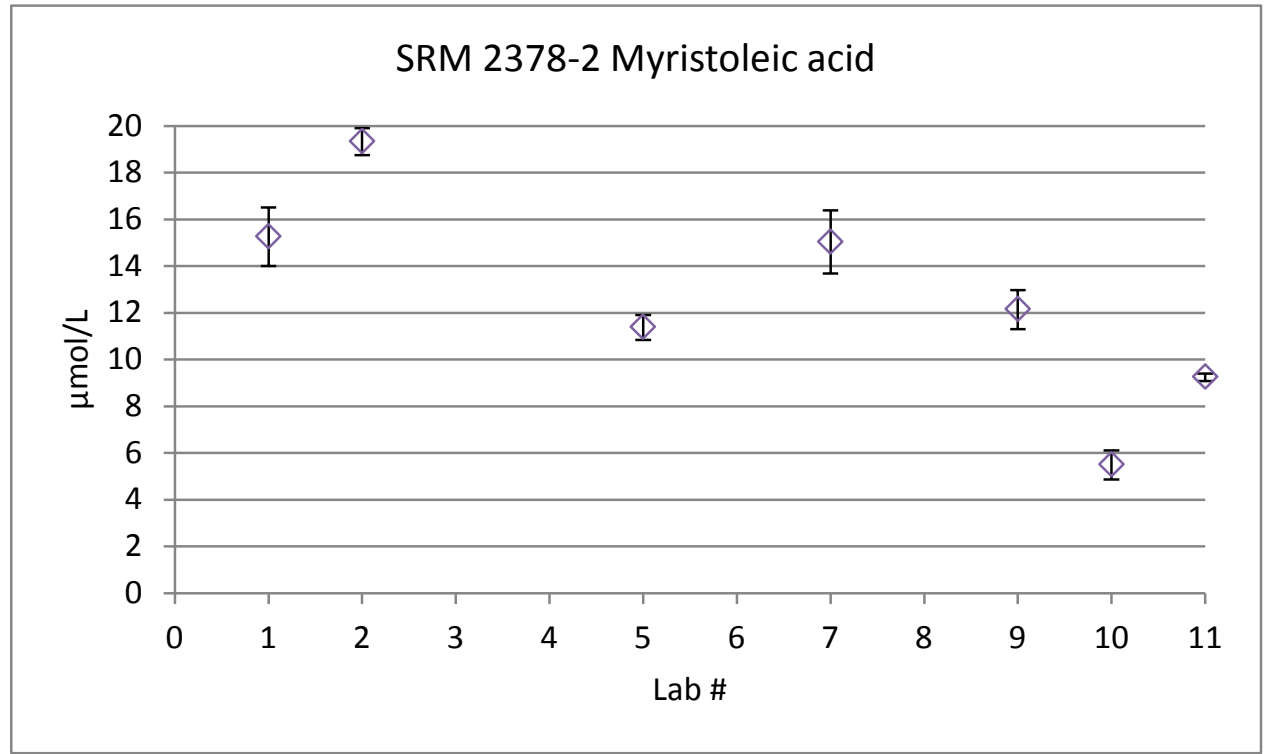

Median $12.1 \mu \mathrm{mol} / \mathrm{L}$ 


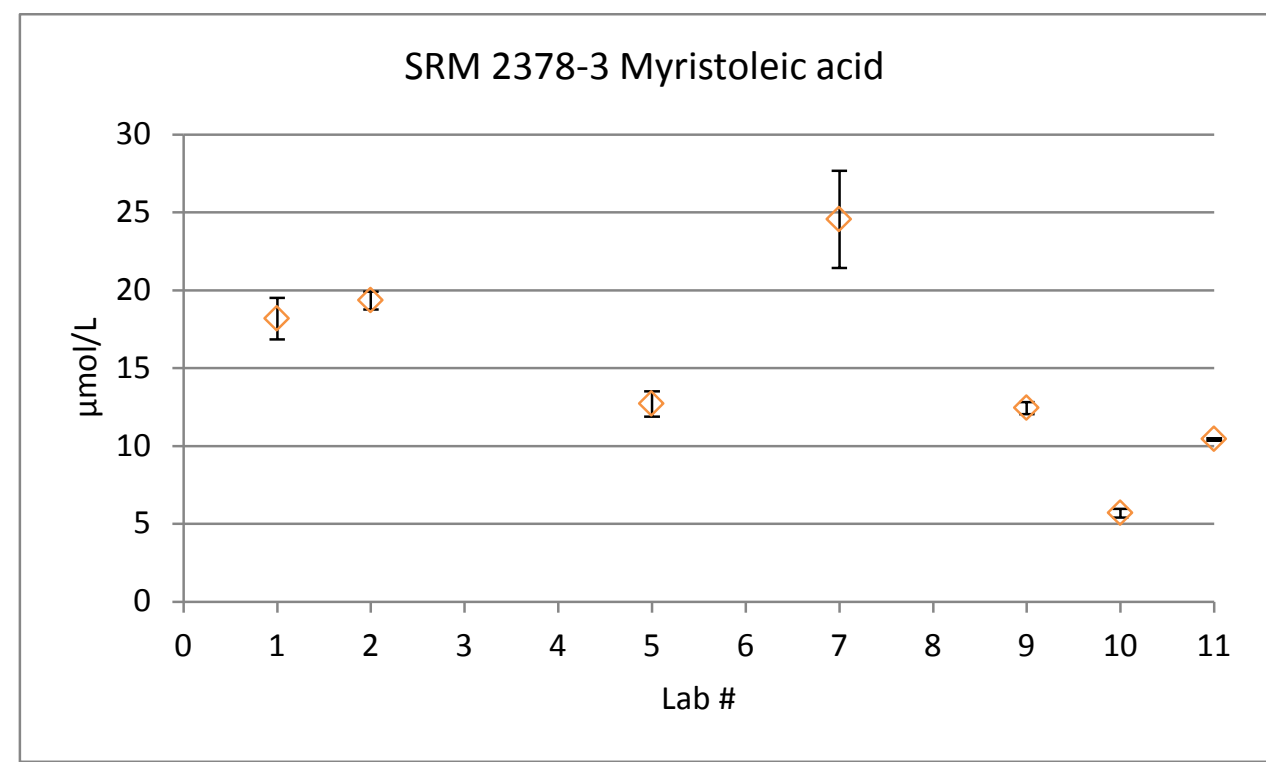

Median $12.7 \mu \mathrm{mol} / \mathrm{L}$

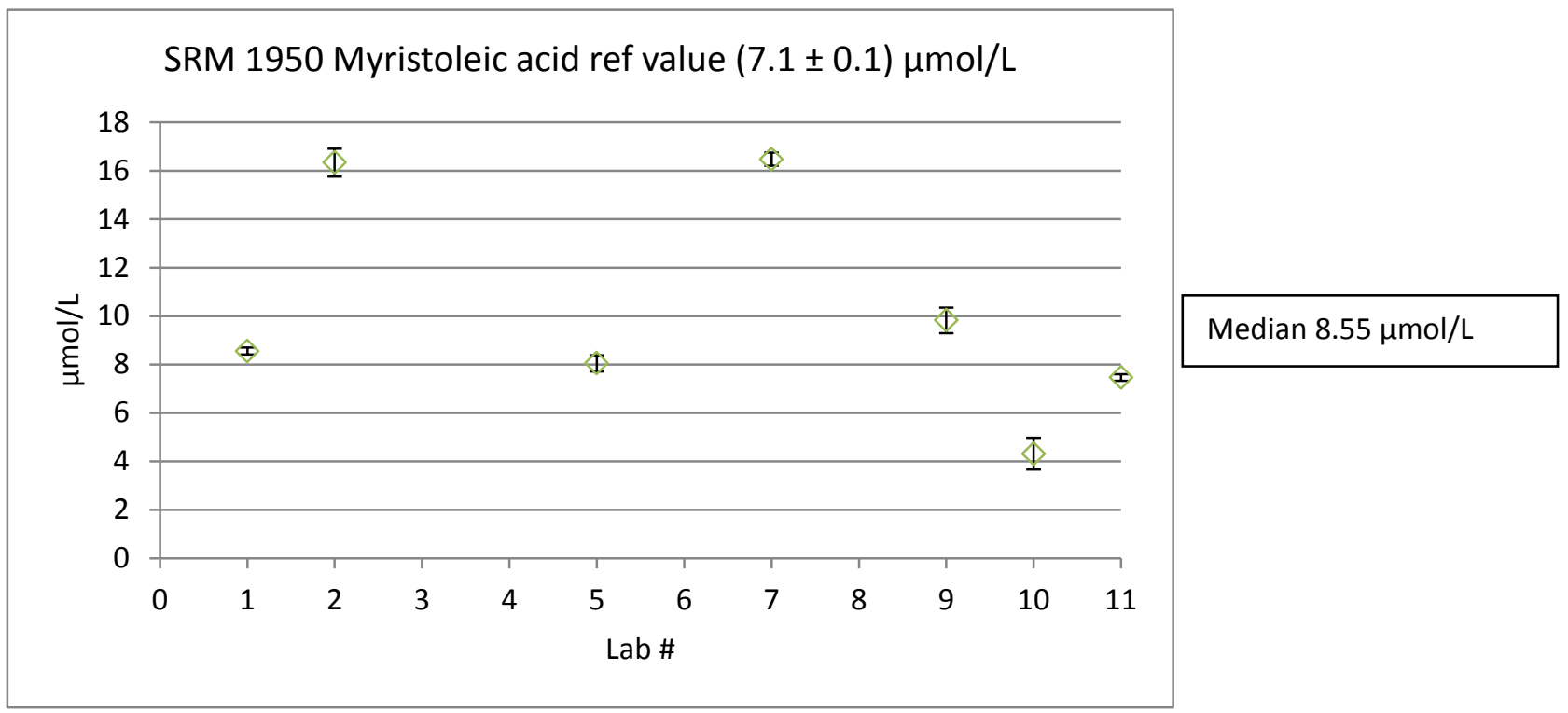




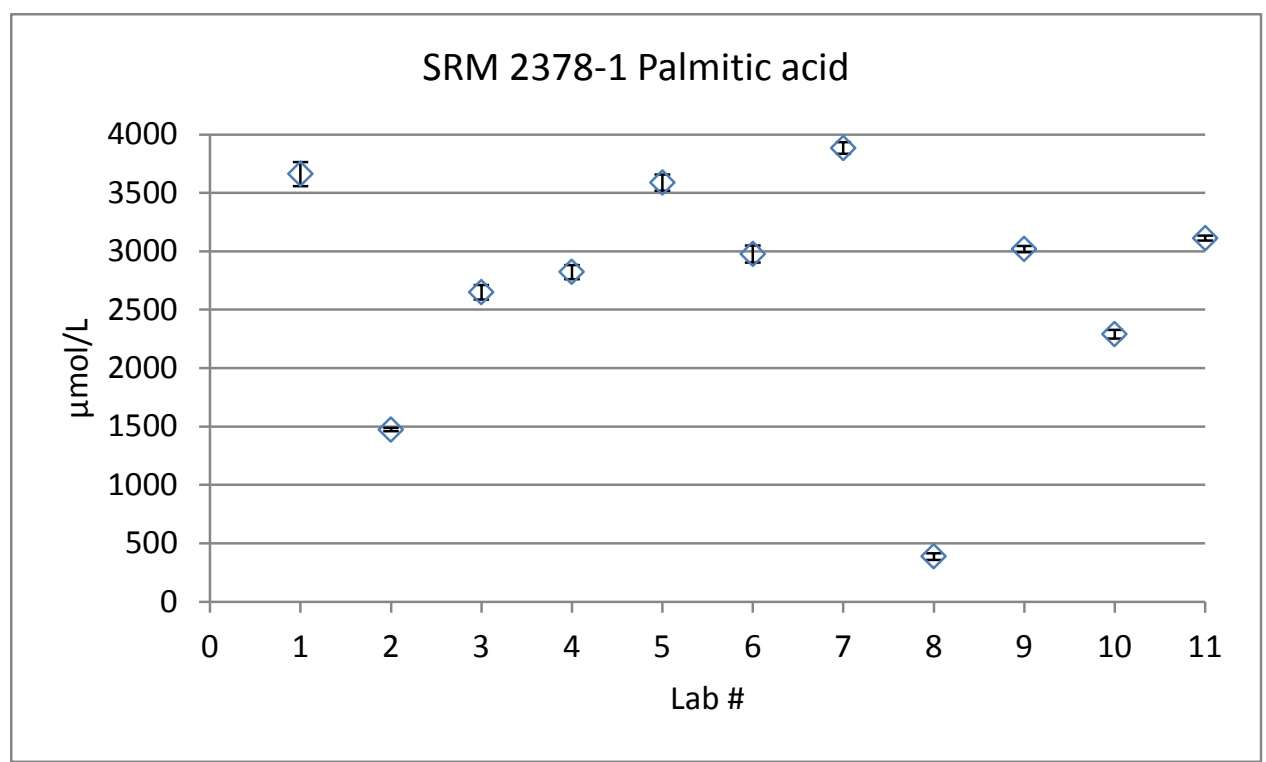

Median (excluding lab 8)

$2900 \mu \mathrm{mol} / \mathrm{L}$

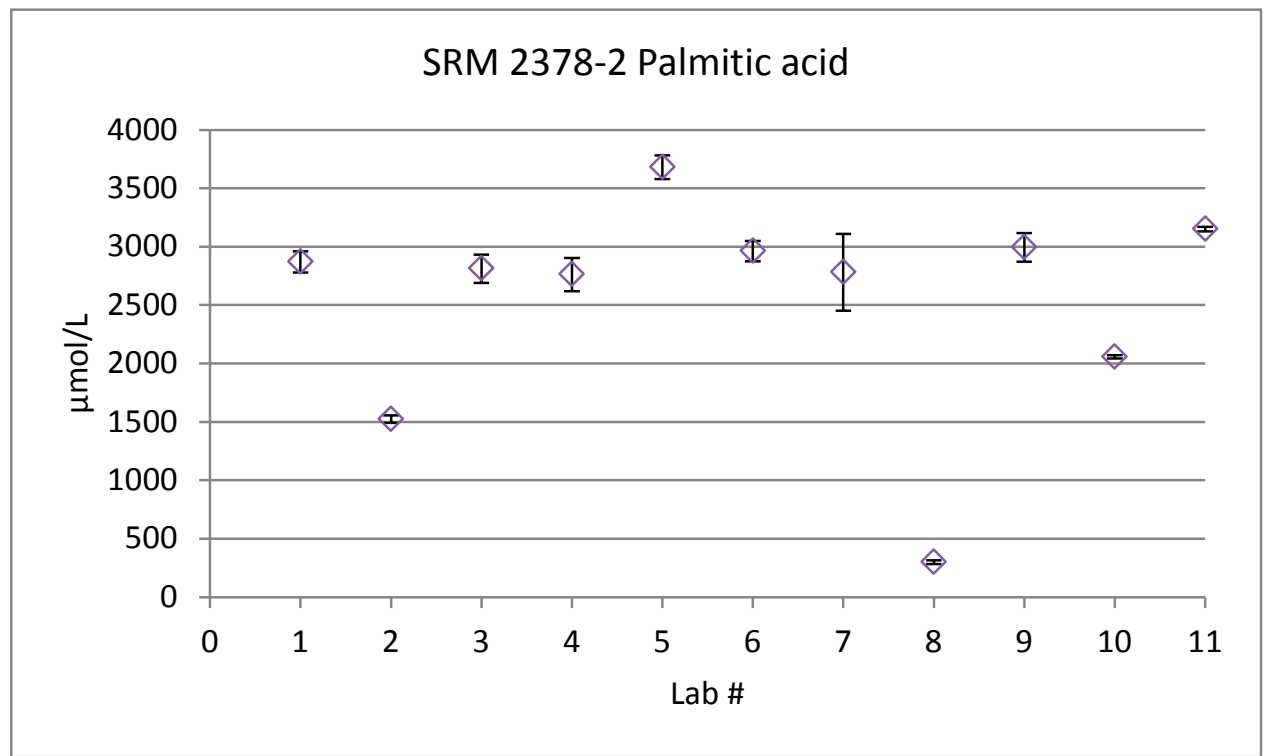

Median (excluding lab 8) $2840 \mu \mathrm{mol} / \mathrm{L}$ 

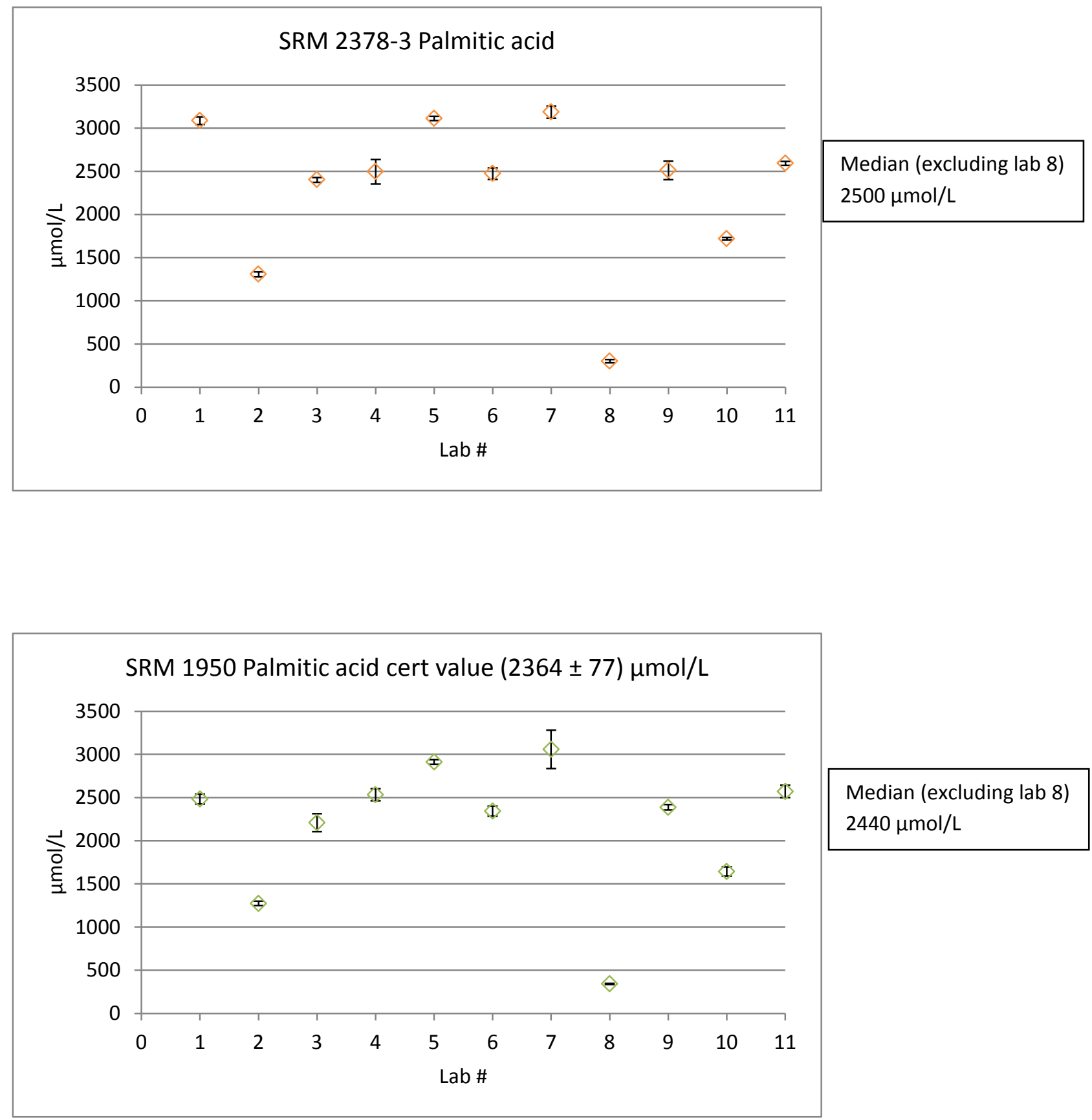

Median (excluding lab 8) $2440 \mu \mathrm{mol} / \mathrm{L}$ 


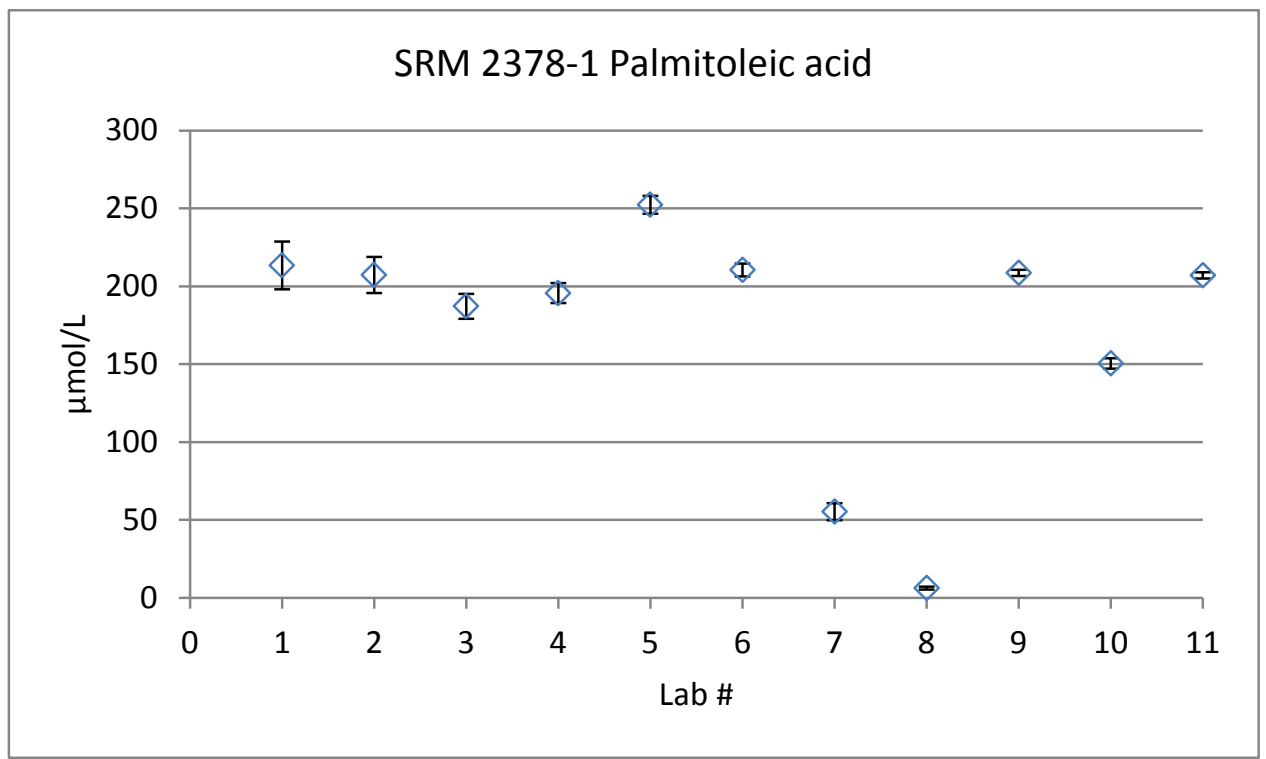

Median (excluding lab 8)

$201 \mu \mathrm{mol} / \mathrm{L}$

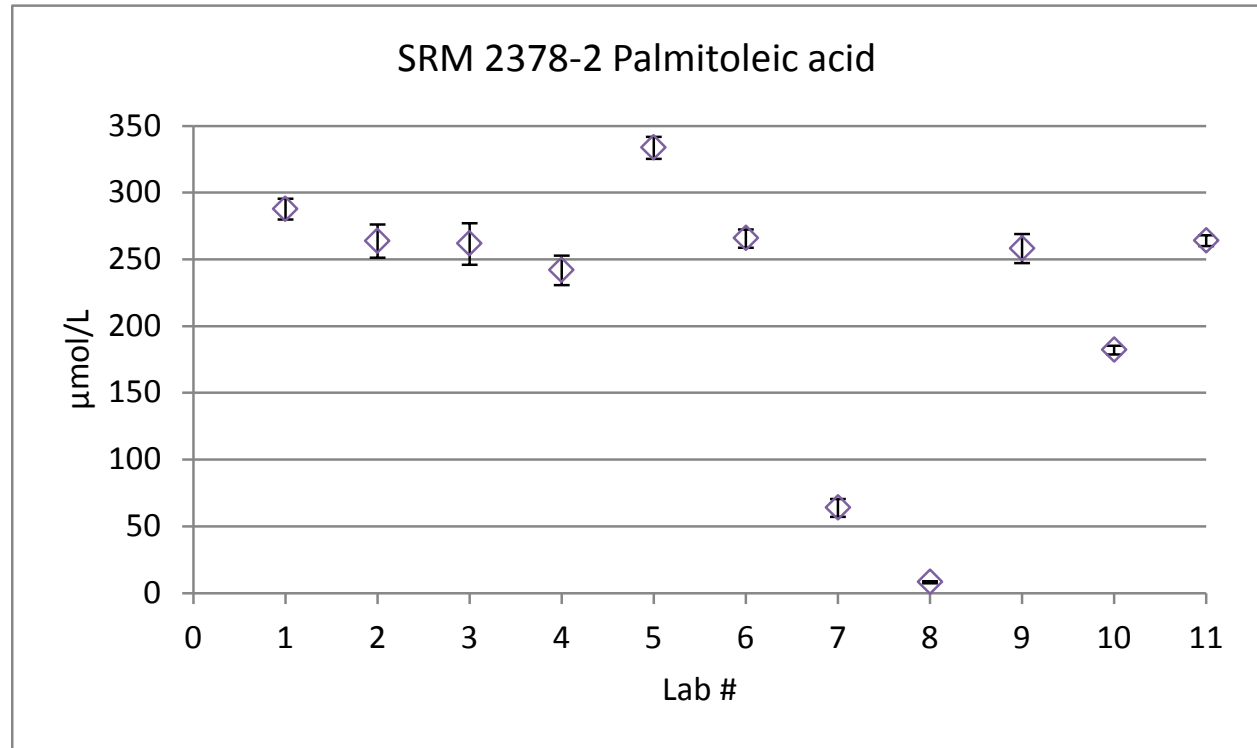

Median (excluding lab 8) $263 \mu \mathrm{mol} / \mathrm{L}$ 

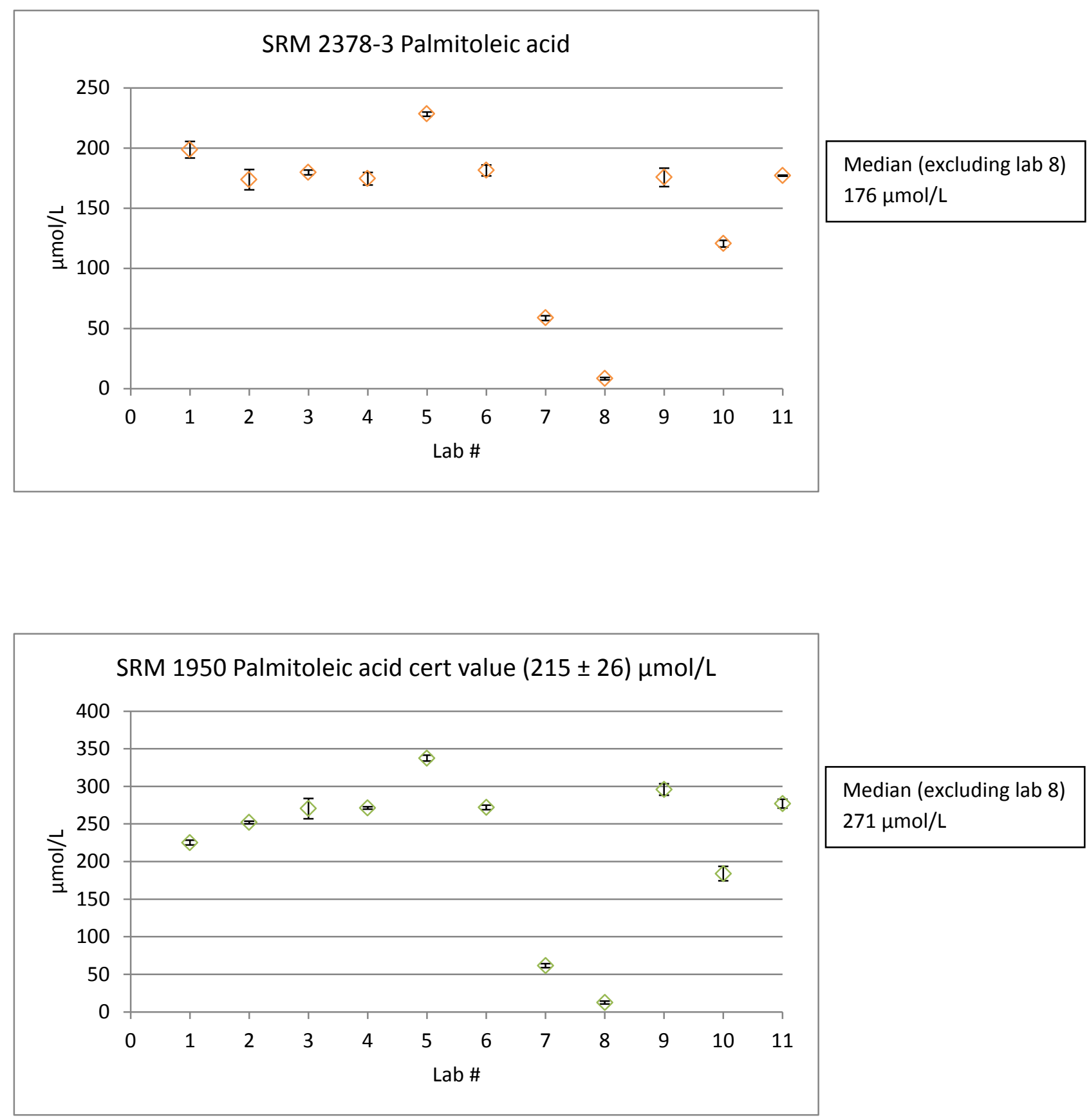

Median (excluding lab 8) $271 \mu \mathrm{mol} / \mathrm{L}$ 


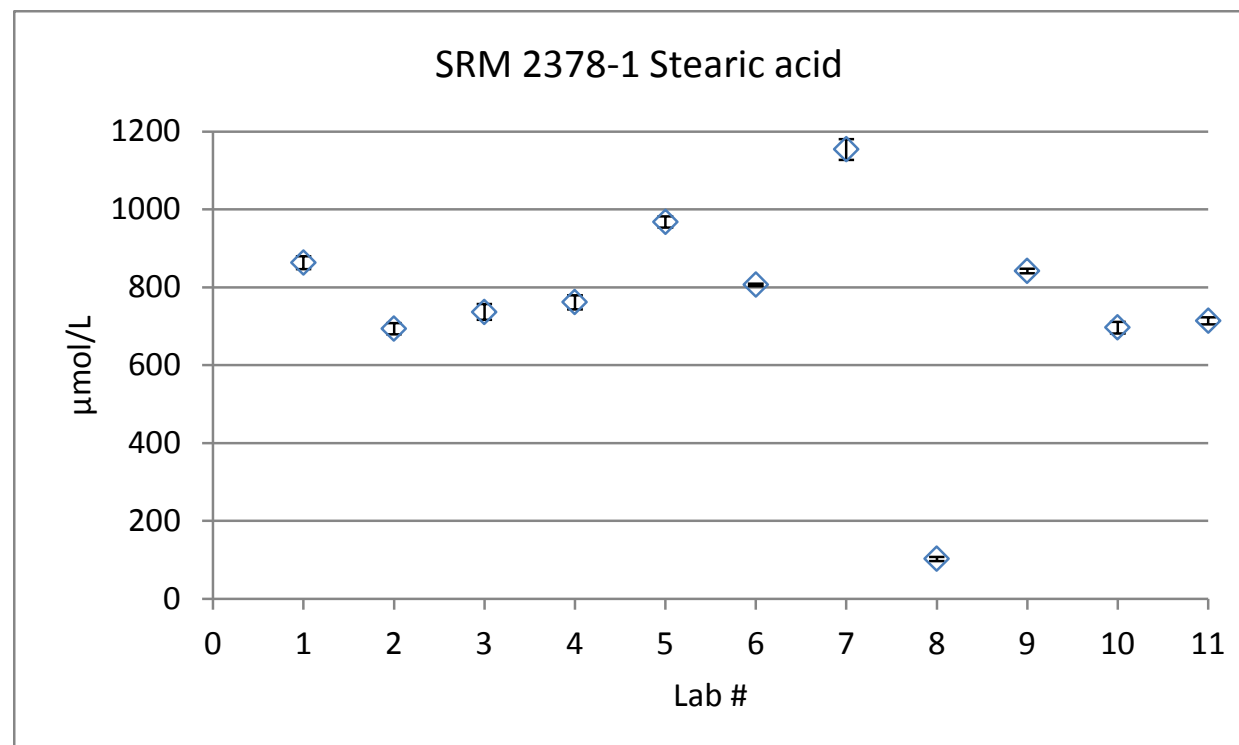

Median (excluding lab 8)

$749 \mu \mathrm{mol} / \mathrm{L}$

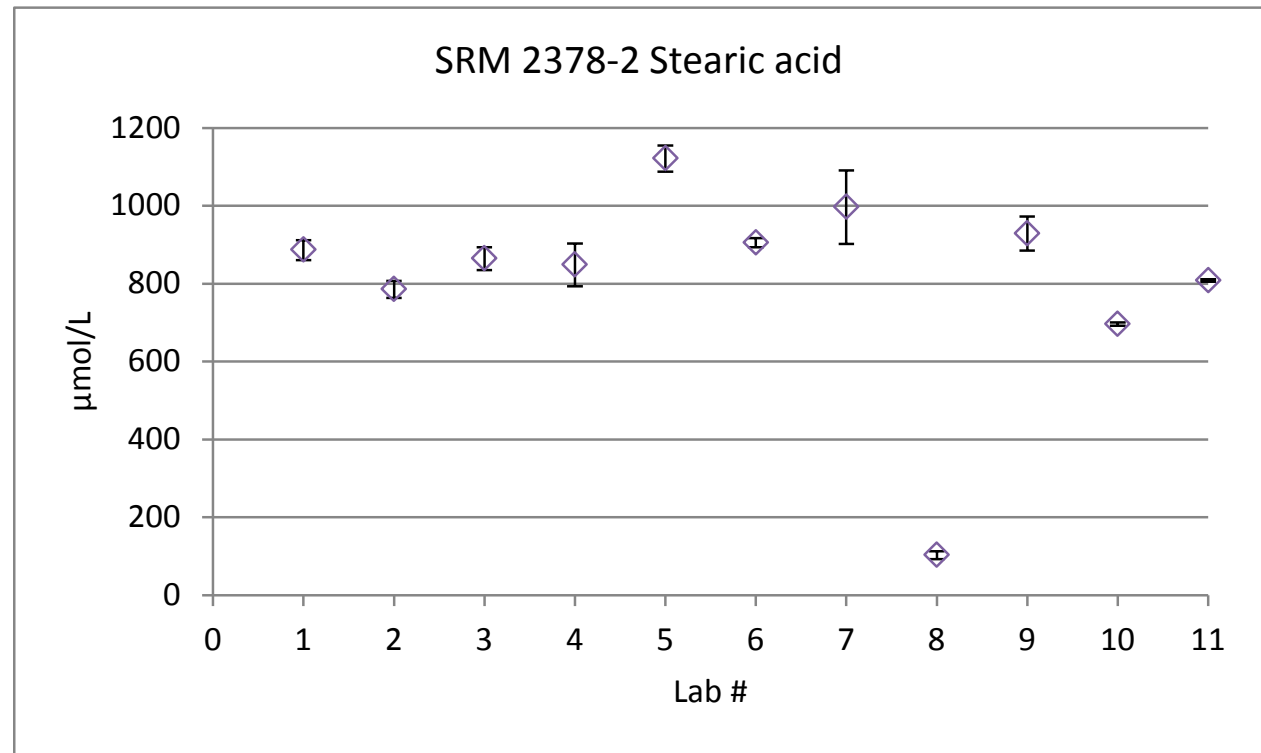

Median (excluding lab 8) $875 \mu \mathrm{mol} / \mathrm{L}$ 

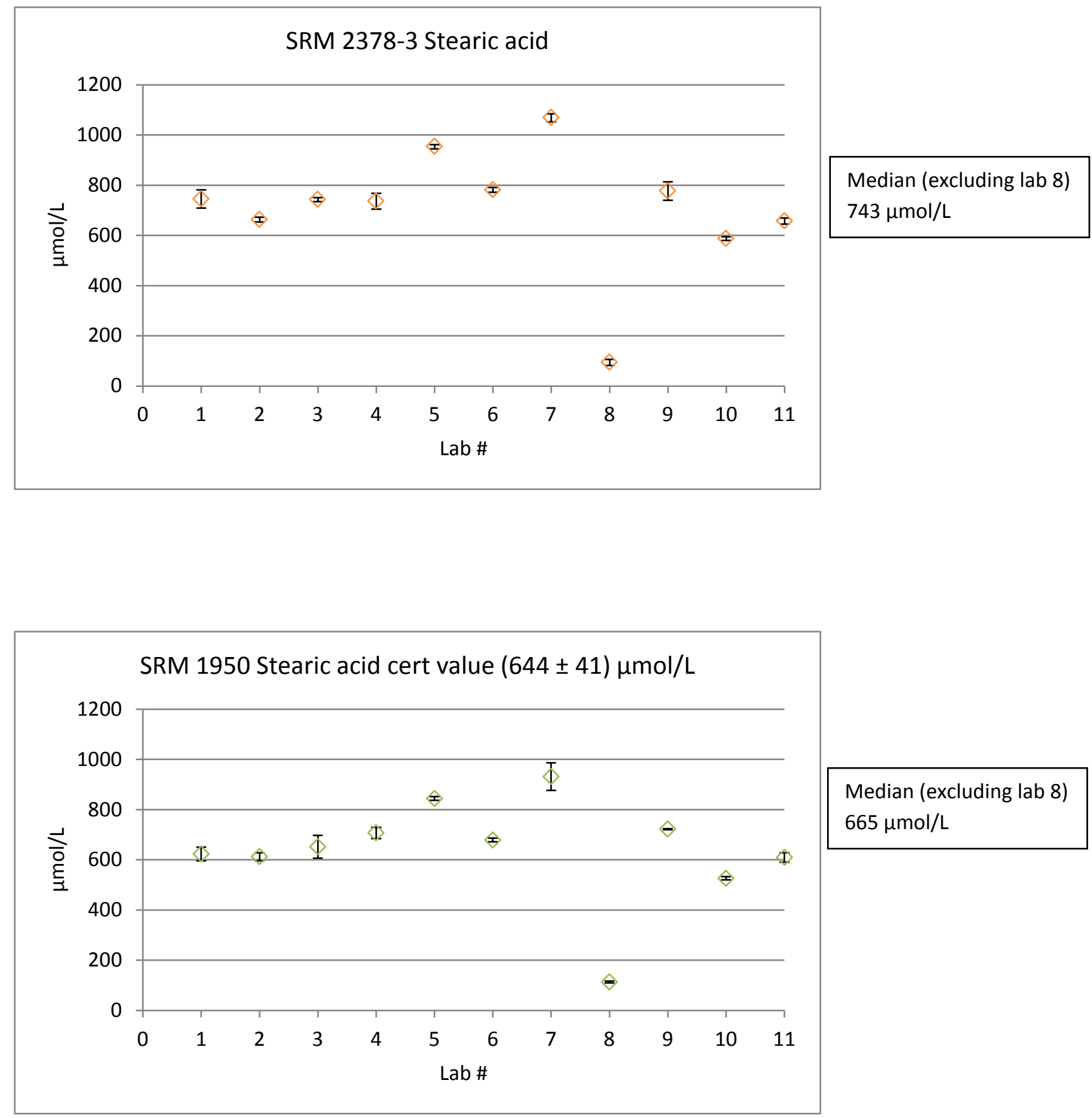

Median (excluding lab 8) $665 \mu \mathrm{mol} / \mathrm{L}$ 


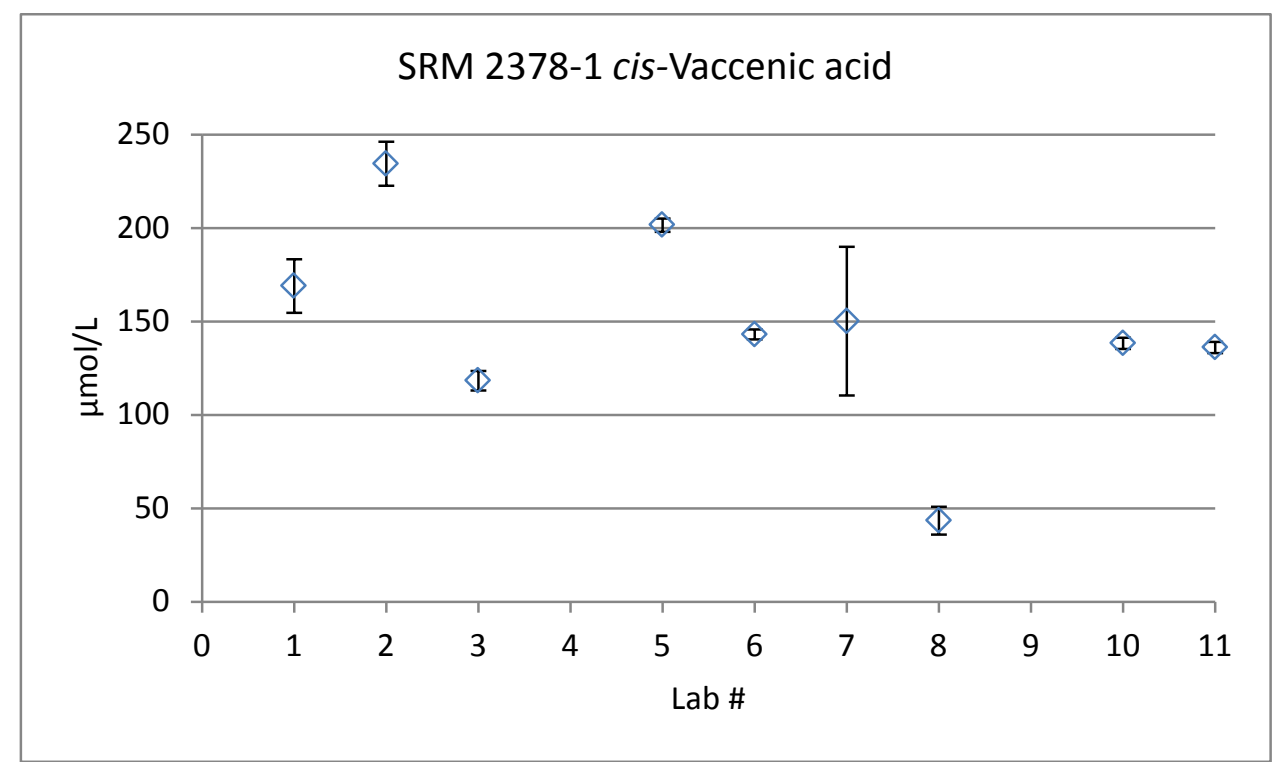

Median (excluding lab 8)

$141 \mu \mathrm{mol} / \mathrm{L}$

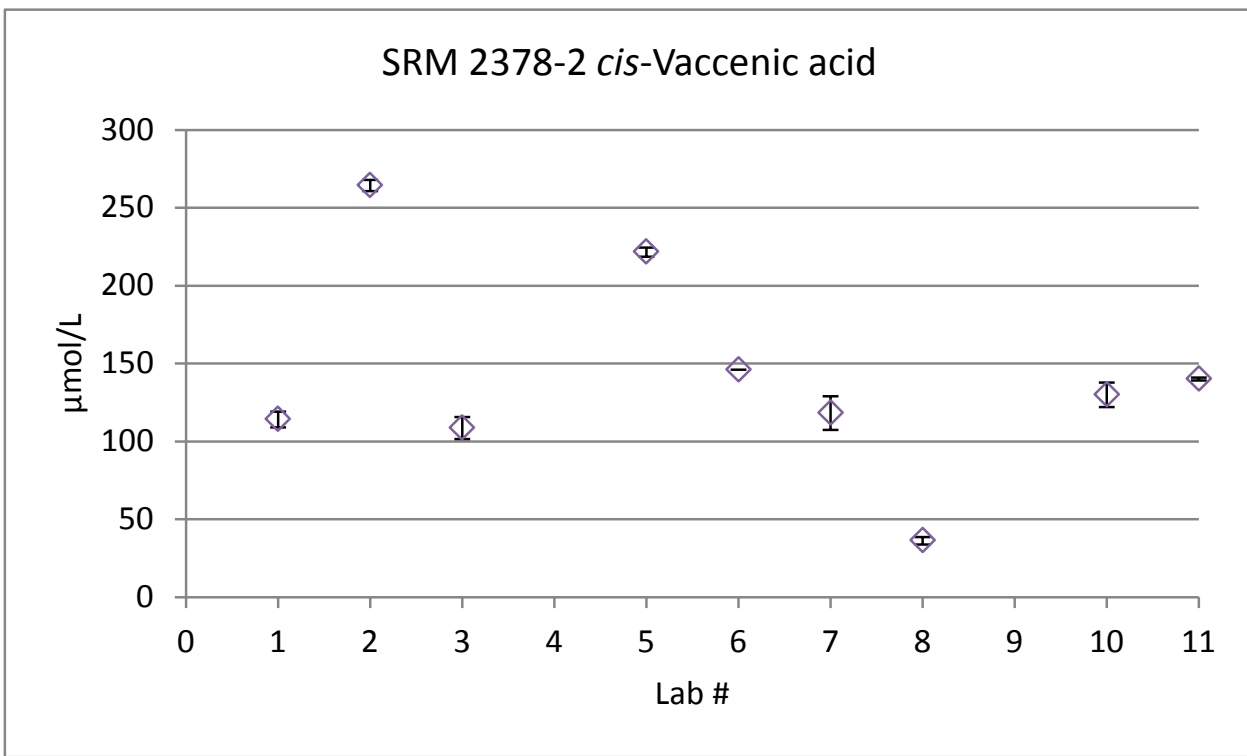

Median (excluding lab 8) $135 \mu \mathrm{mol} / \mathrm{L}$ 


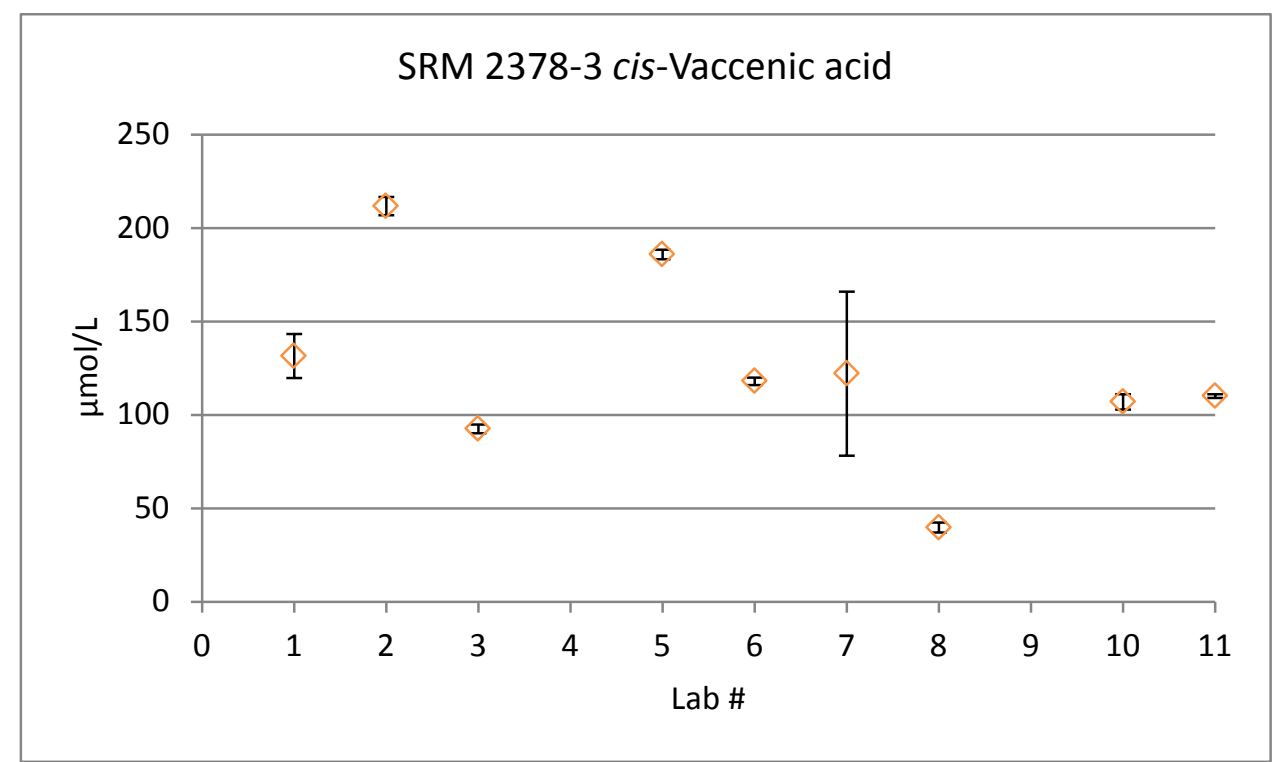

Median (excluding lab 8) $120 \mu \mathrm{mol} / \mathrm{L}$

SRM 1950 cis-Vaccenic acid ref value (136 \pm 3$) \mu \mathrm{mol} / \mathrm{L}$

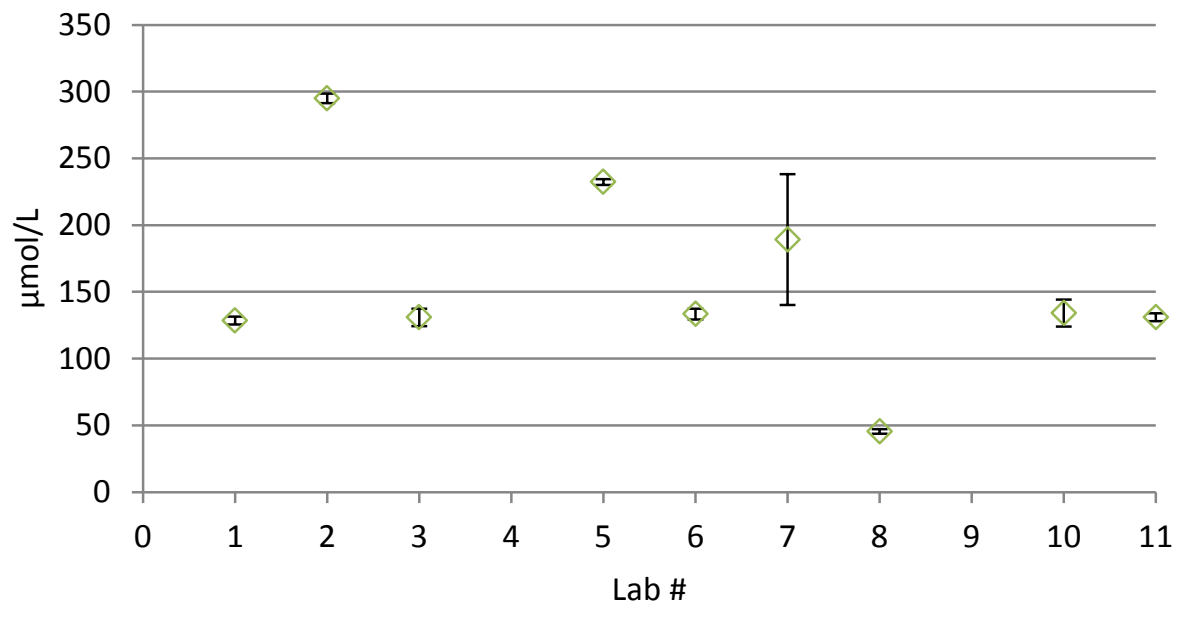

Median (excluding lab 8)

$134 \mu \mathrm{mol} / \mathrm{L}$ 


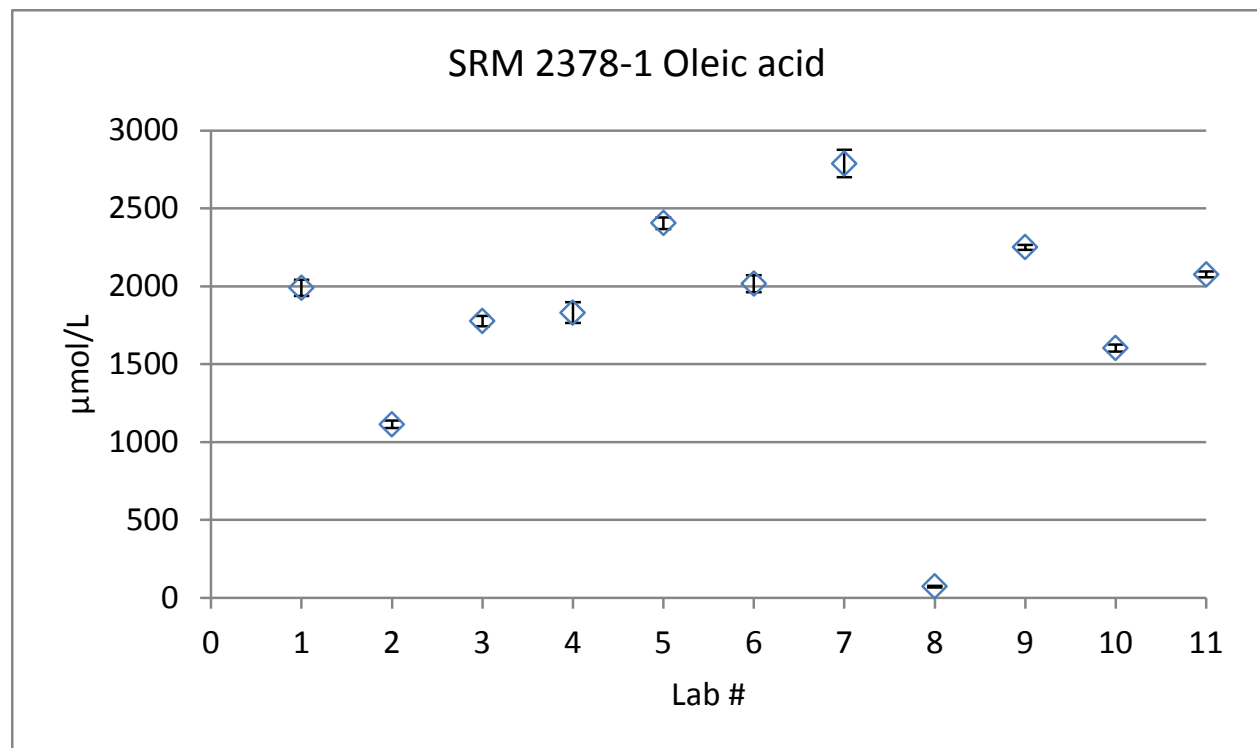

Median (excluding lab 8) $1910 \mu \mathrm{mol} / \mathrm{L}$

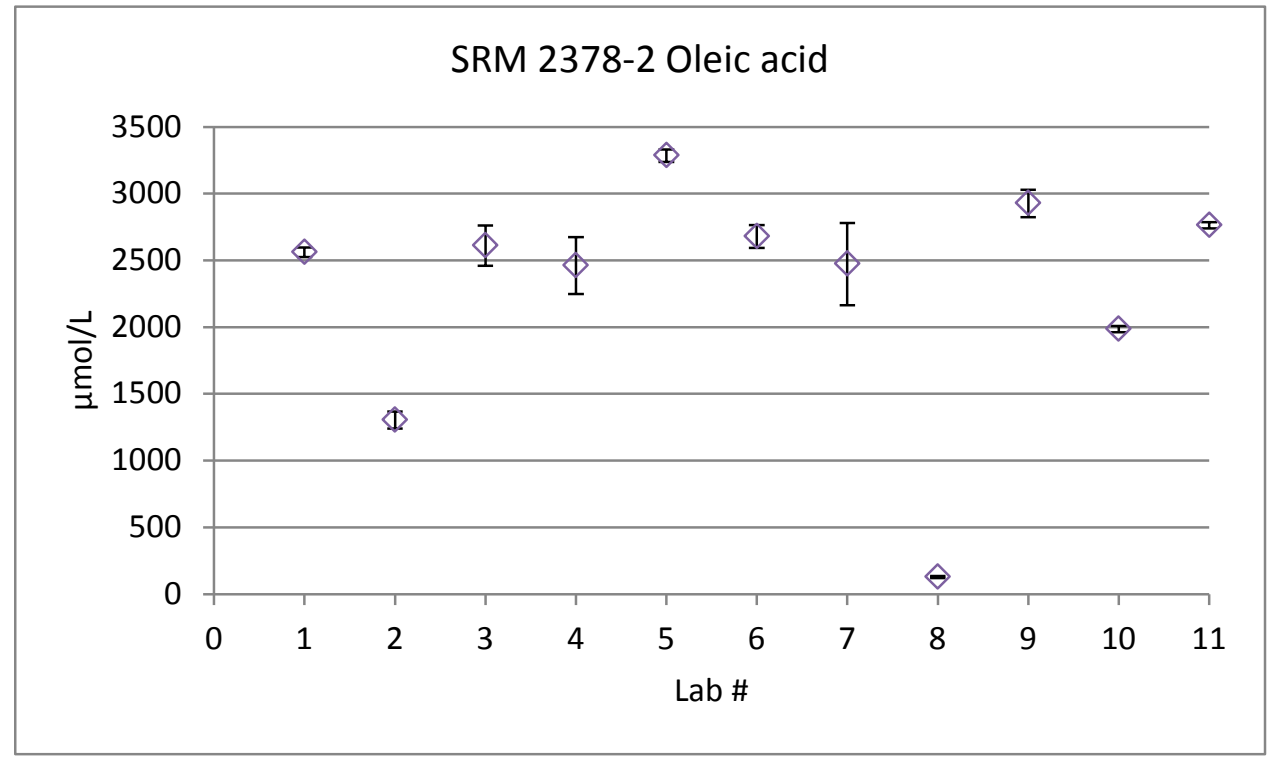

Median (excluding lab 8) $2590 \mu \mathrm{mol} / \mathrm{L}$ 

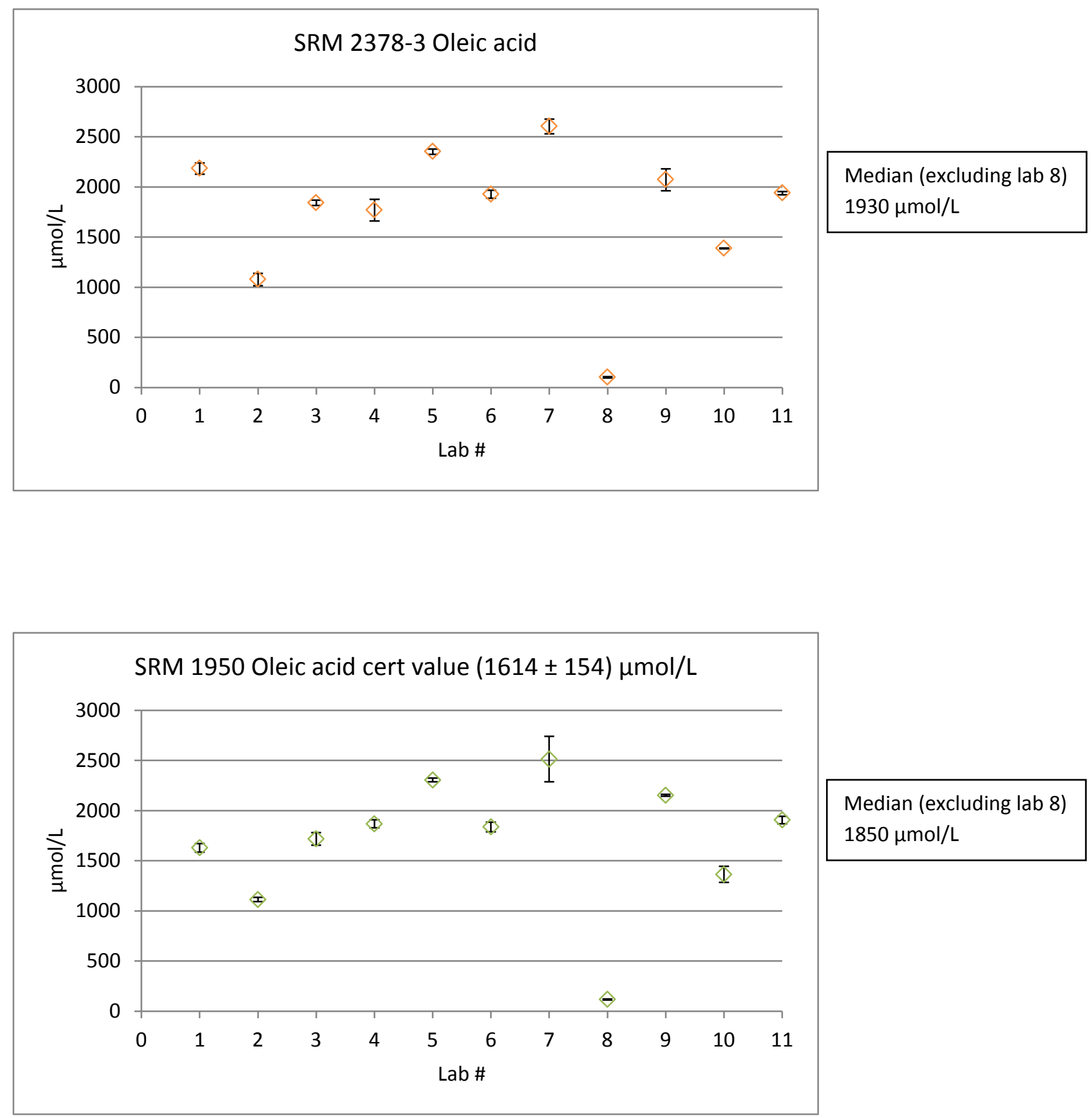

Median (excluding lab 8) $1850 \mu \mathrm{mol} / \mathrm{L}$ 


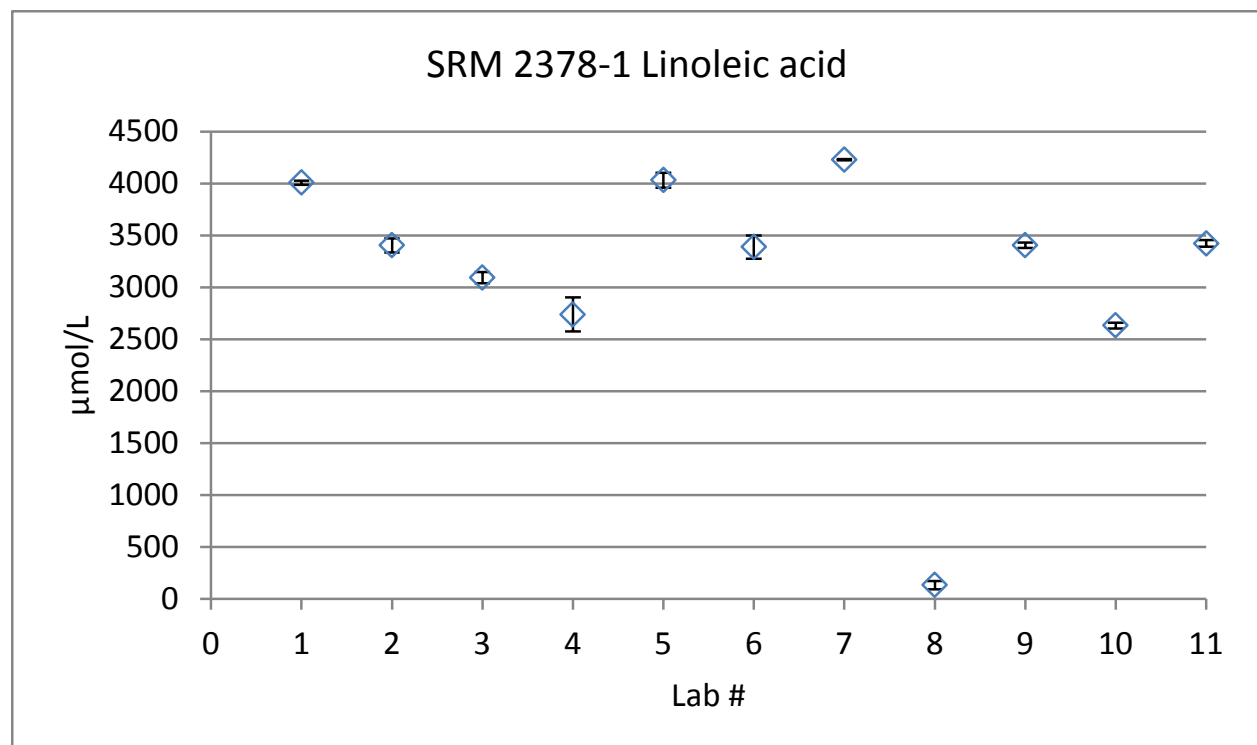

Median (excluding lab 8) $3400 \mu \mathrm{mol} / \mathrm{L}$

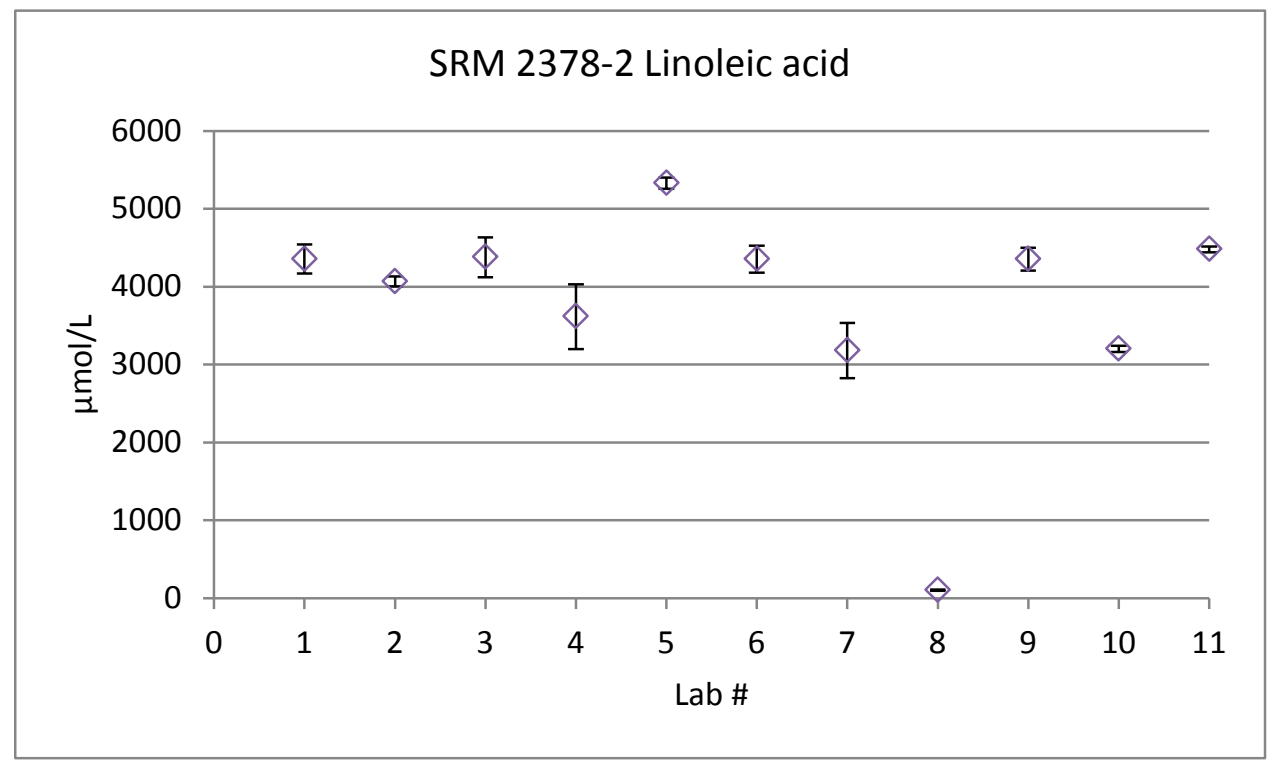

Median (excluding lab 8) $4350 \mu \mathrm{mol} / \mathrm{L}$ 


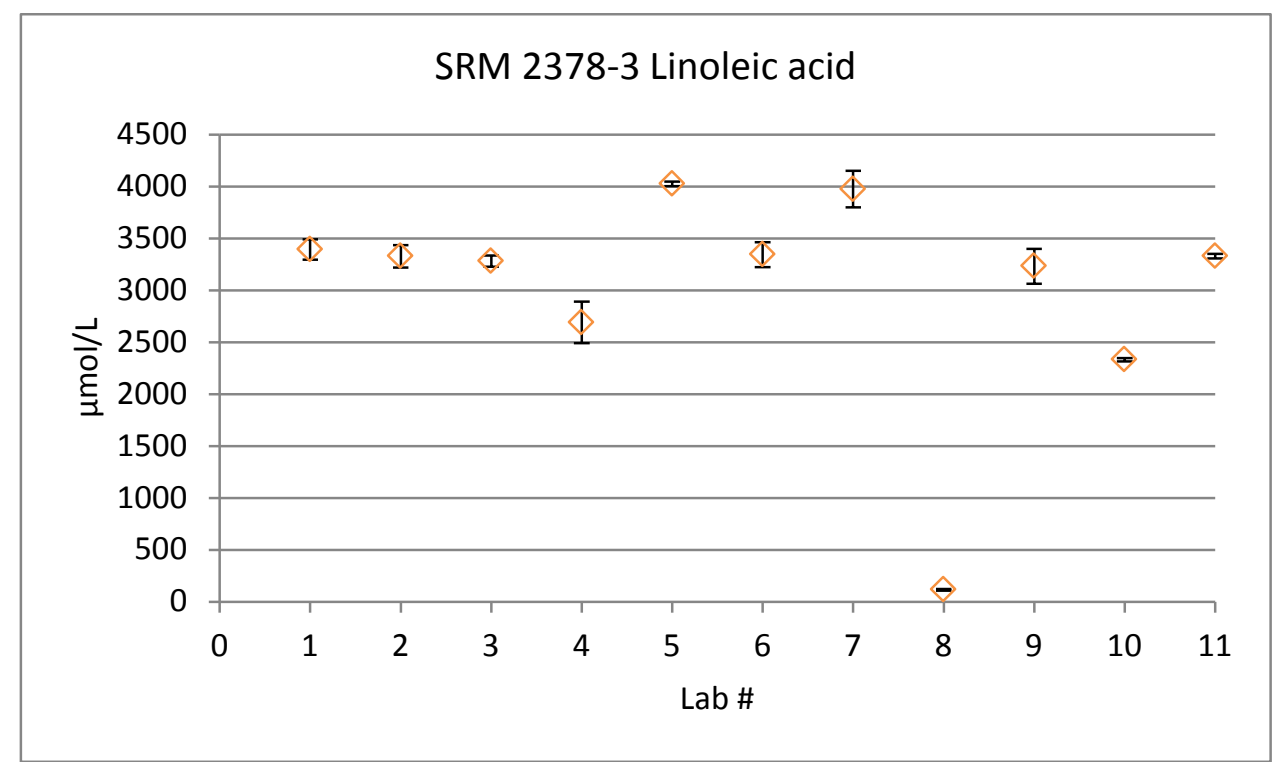

Median (excluding lab 8) $3330 \mu \mathrm{mol} / \mathrm{L}$

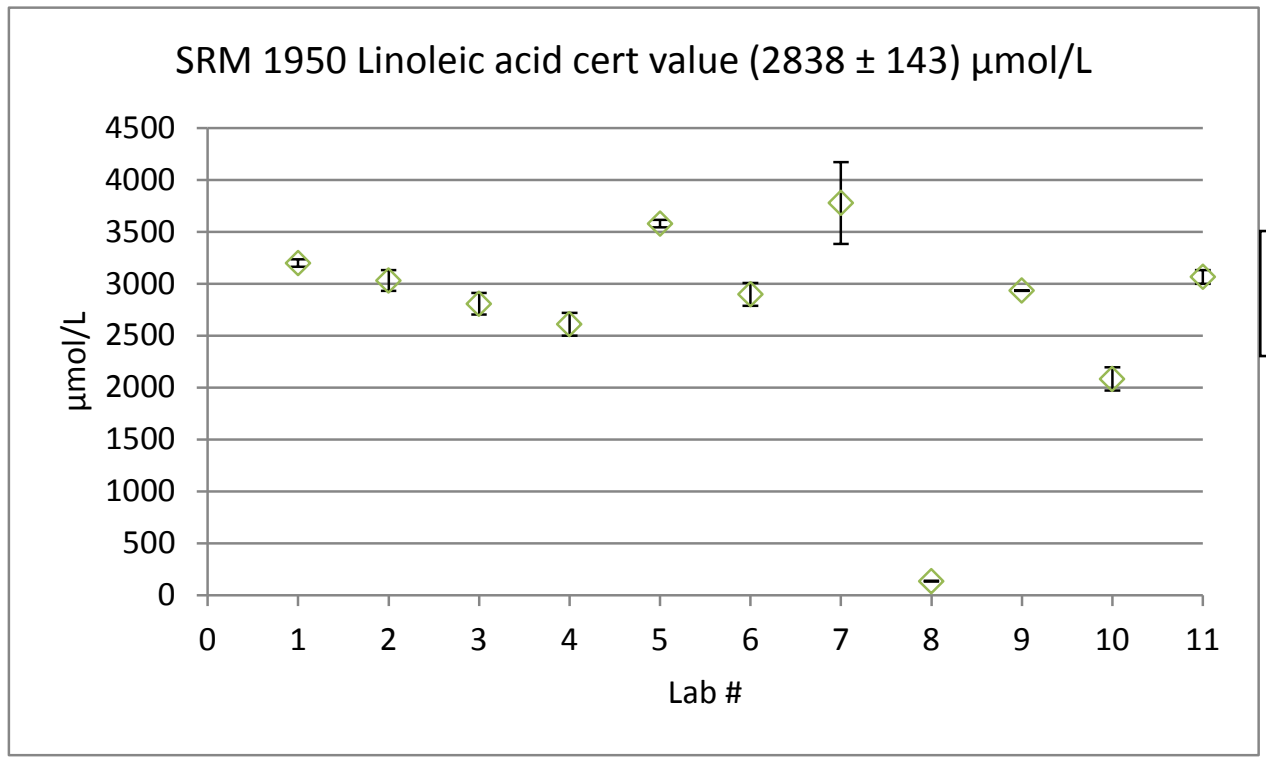

Median (excluding lab 8) $2980 \mu \mathrm{mol} / \mathrm{L}$ 


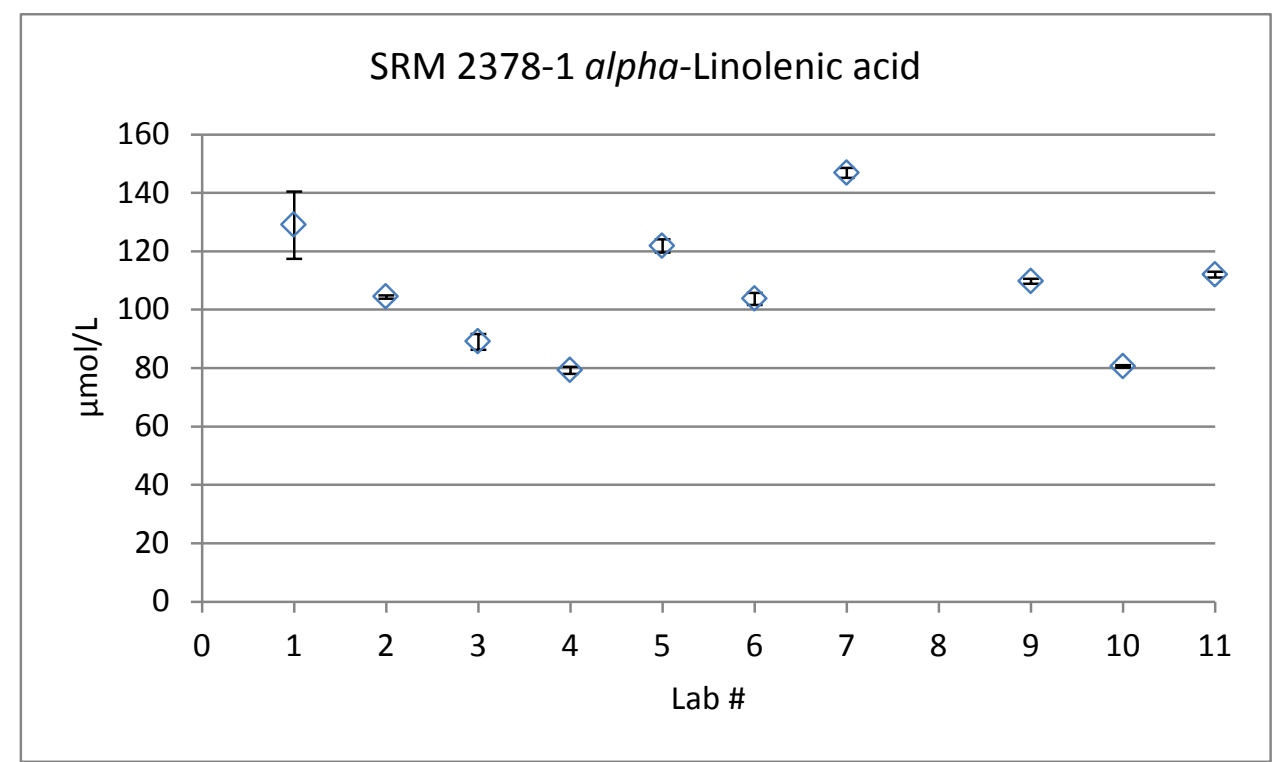

Median $104 \mu \mathrm{mol} / \mathrm{L}$

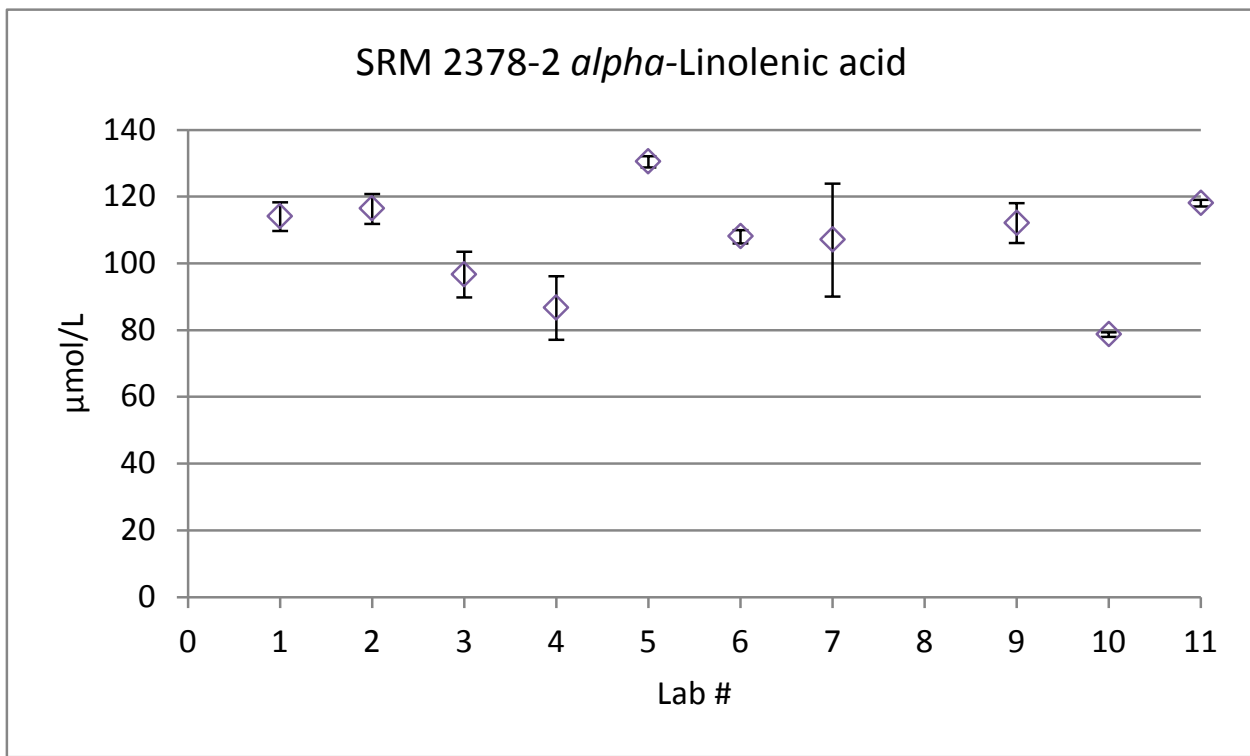

Median $110 \mu \mathrm{mol} / \mathrm{L}$ 

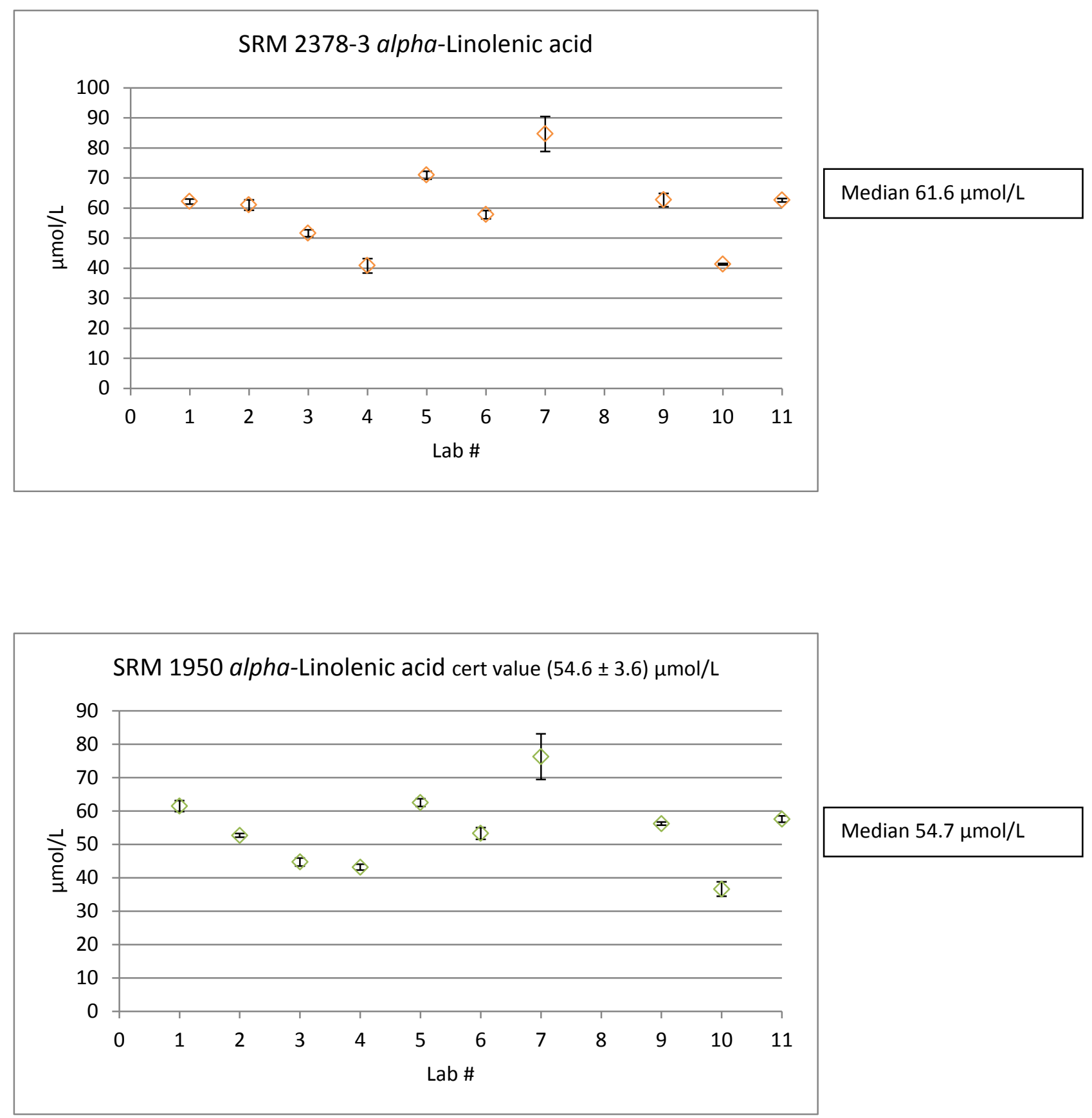

Median $54.7 \mu \mathrm{mol} / \mathrm{L}$ 

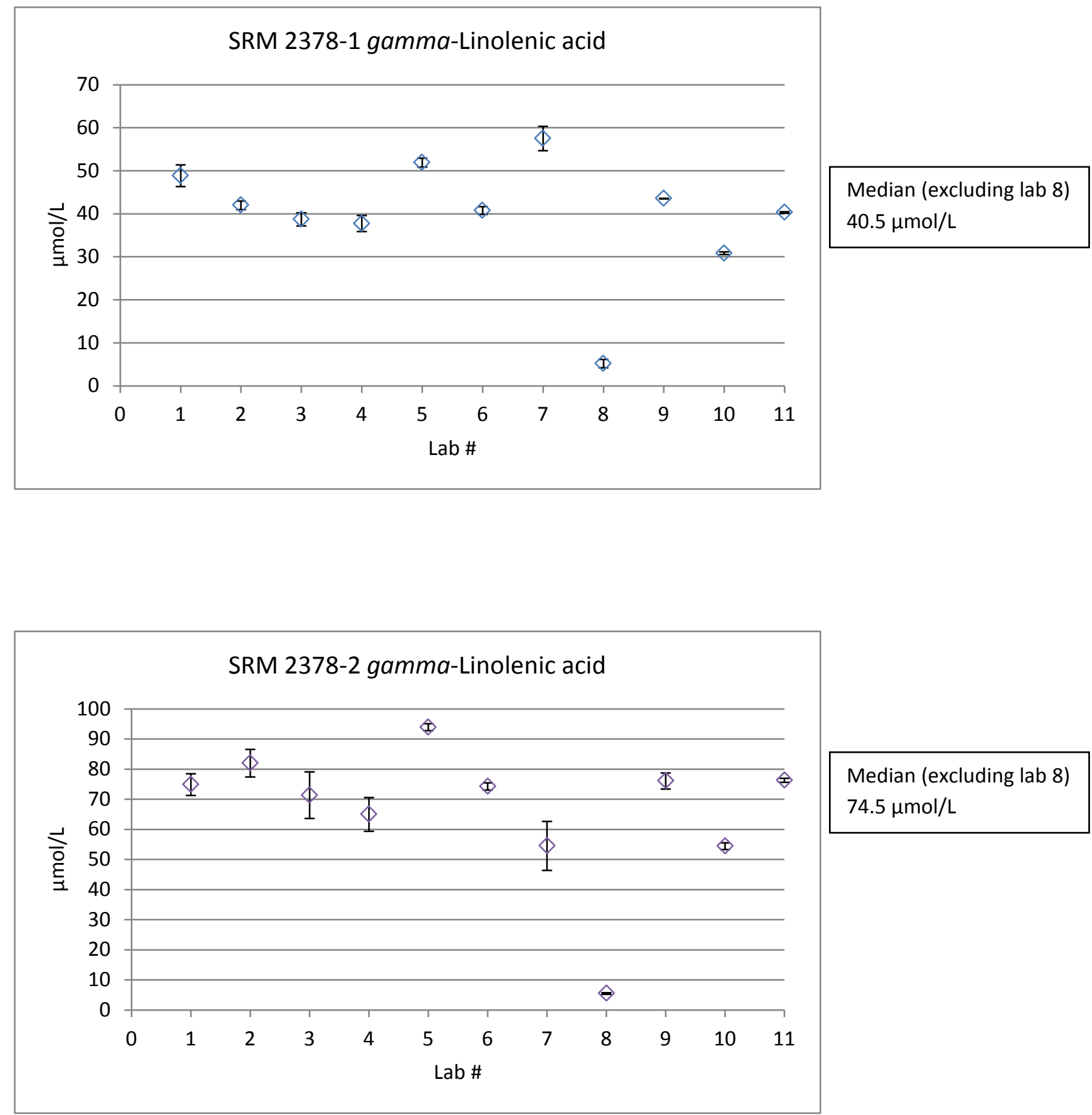

Median (excluding lab 8)

$74.5 \mu \mathrm{mol} / \mathrm{L}$ 


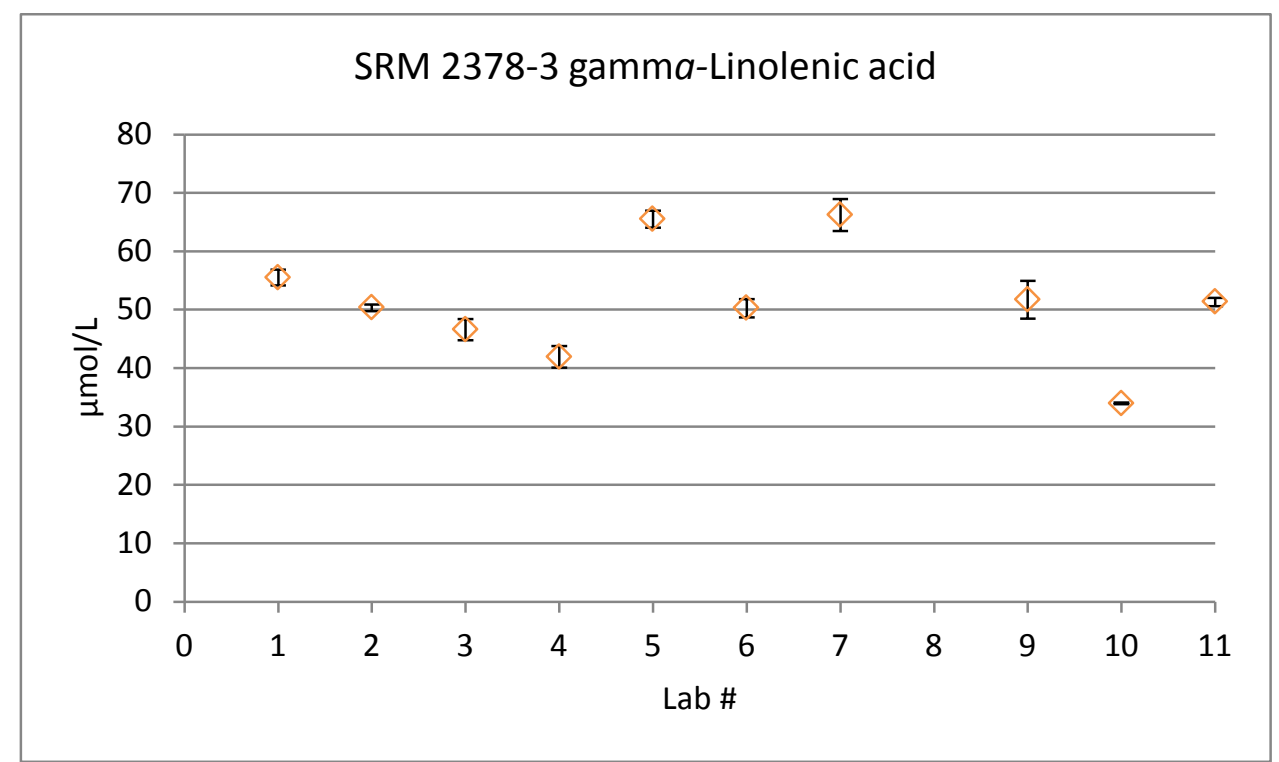

Median (excluding lab 8)

$50.8 \mu \mathrm{mol} / \mathrm{L}$

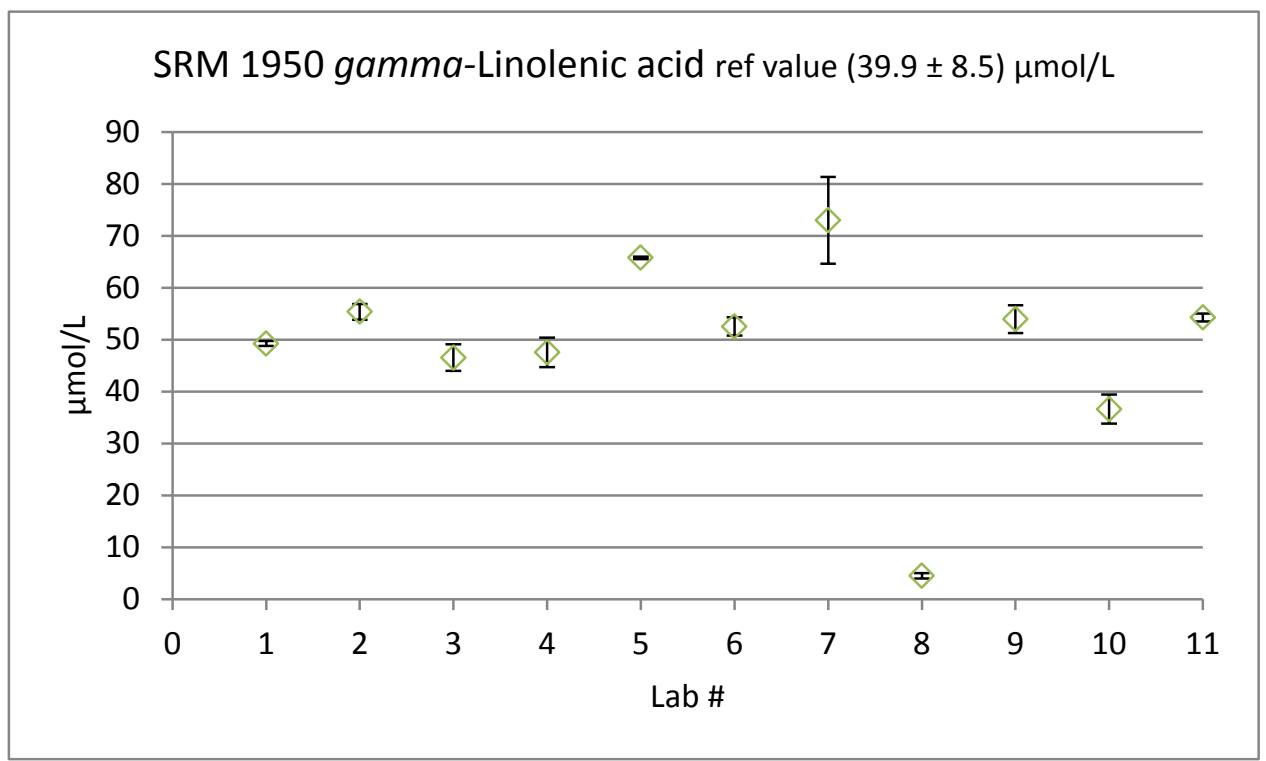

Median (excluding lab 8)

$53.2 \mu \mathrm{mol} / \mathrm{L}$ 


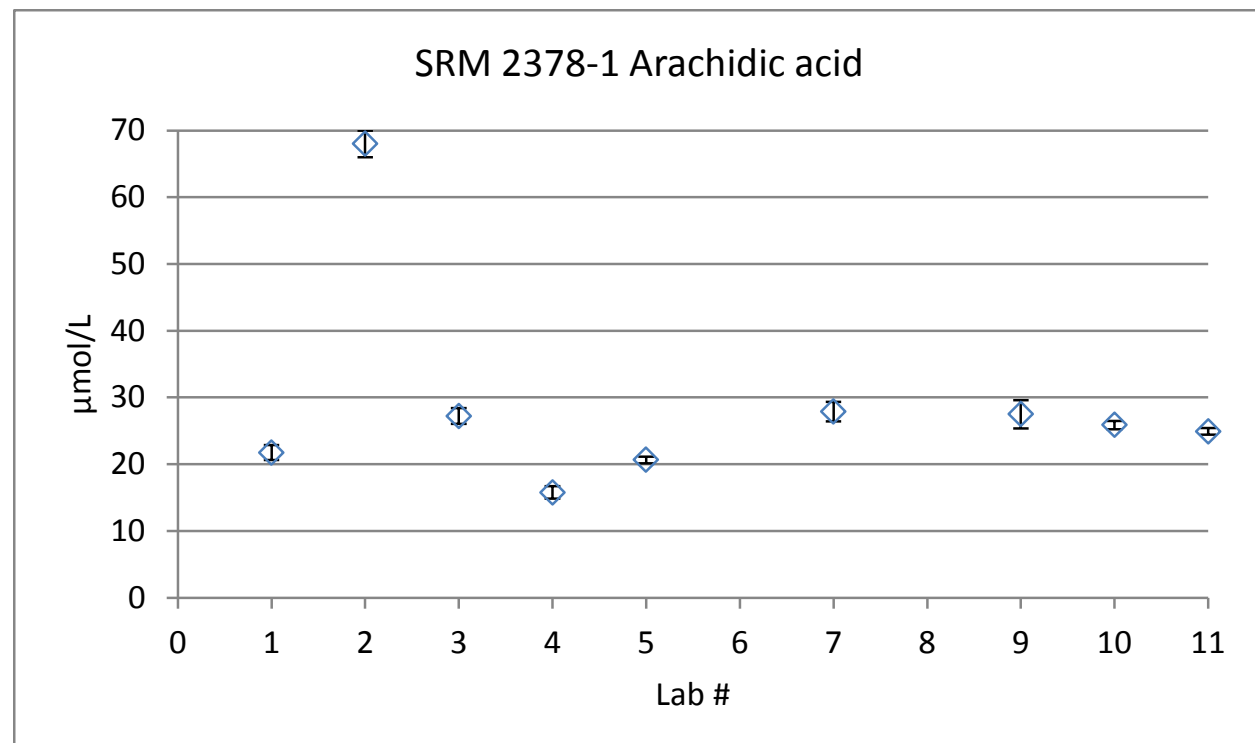

Median $24.9 \mu \mathrm{mol} / \mathrm{L}$

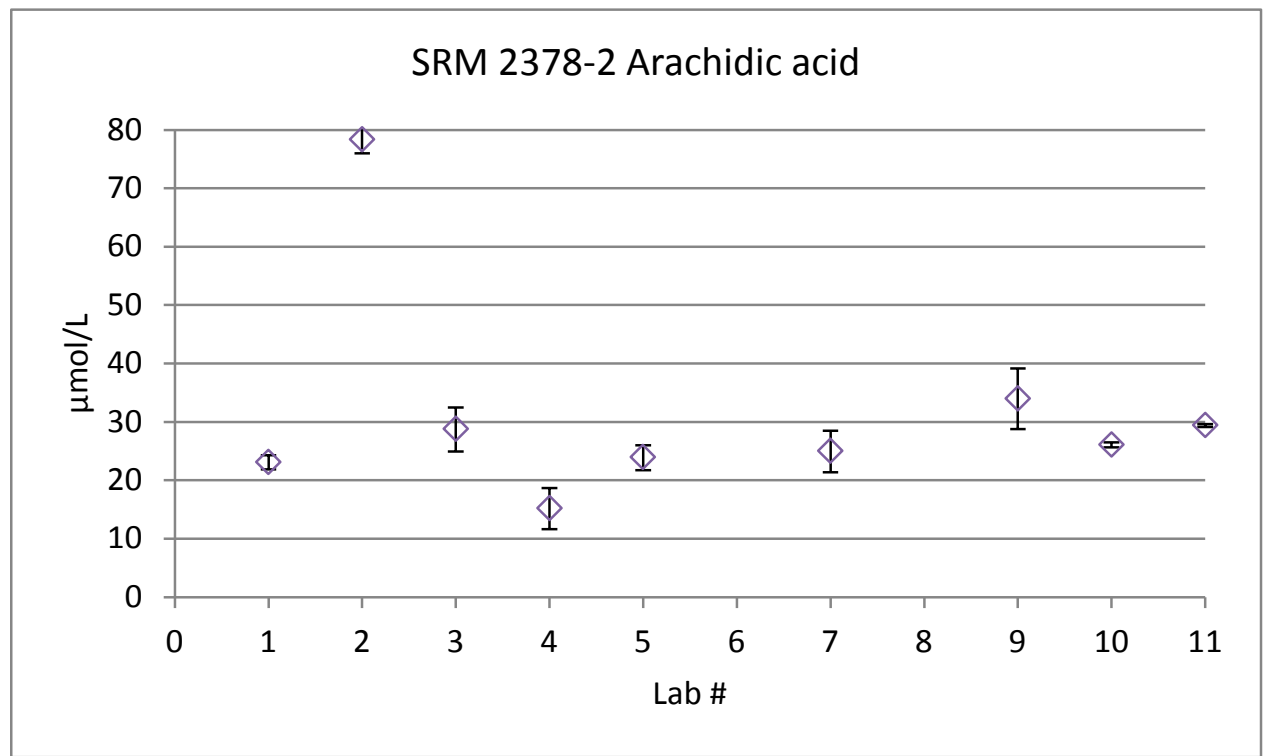

Median $26.1 \mu \mathrm{mol} / \mathrm{L}$ 

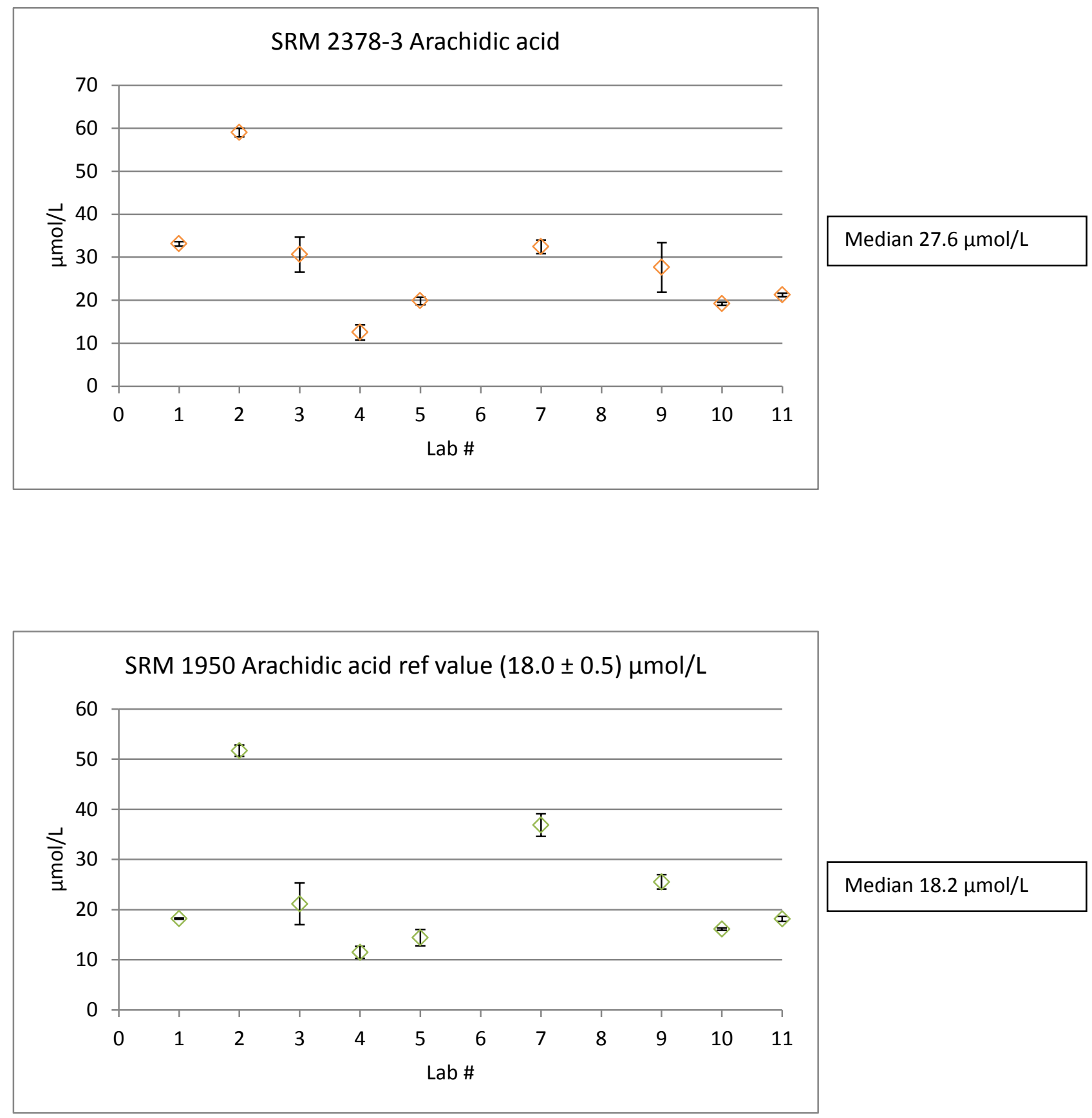

Median $18.2 \mu \mathrm{mol} / \mathrm{L}$ 


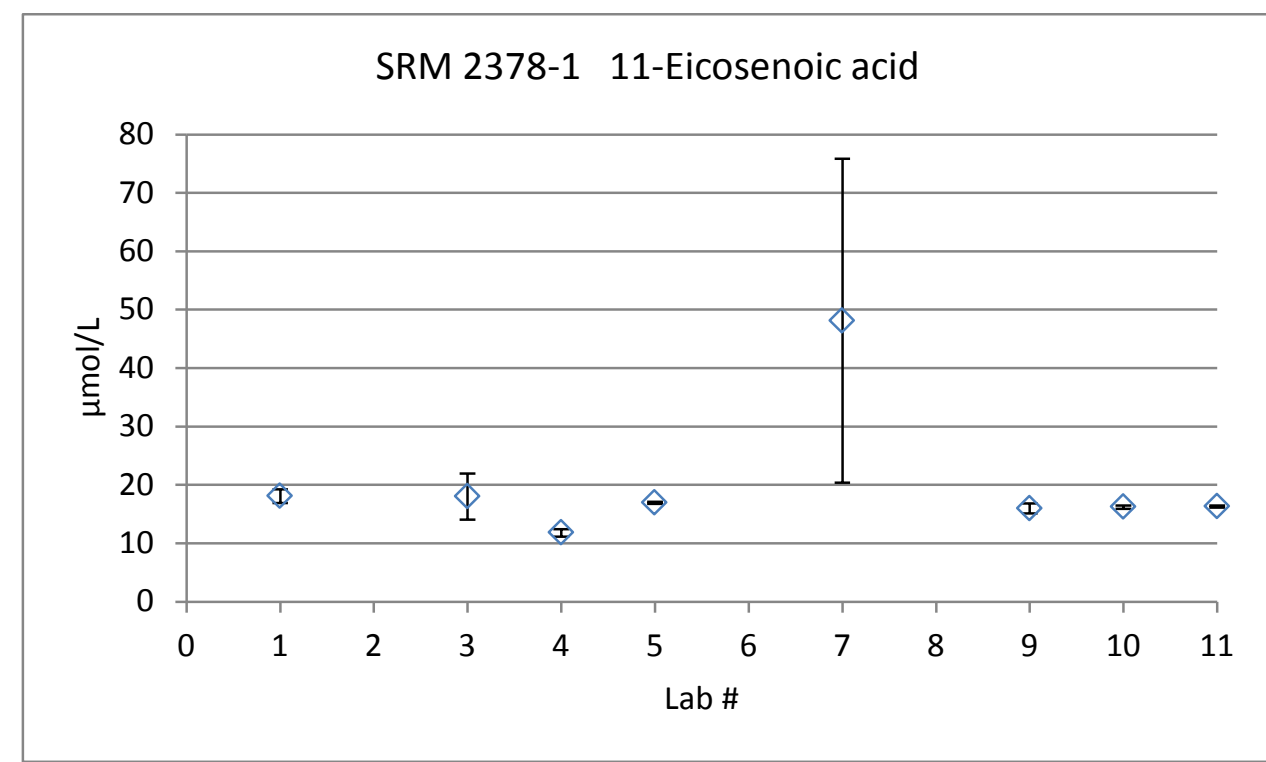

Median $16.3 \mu \mathrm{mol} / \mathrm{L}$

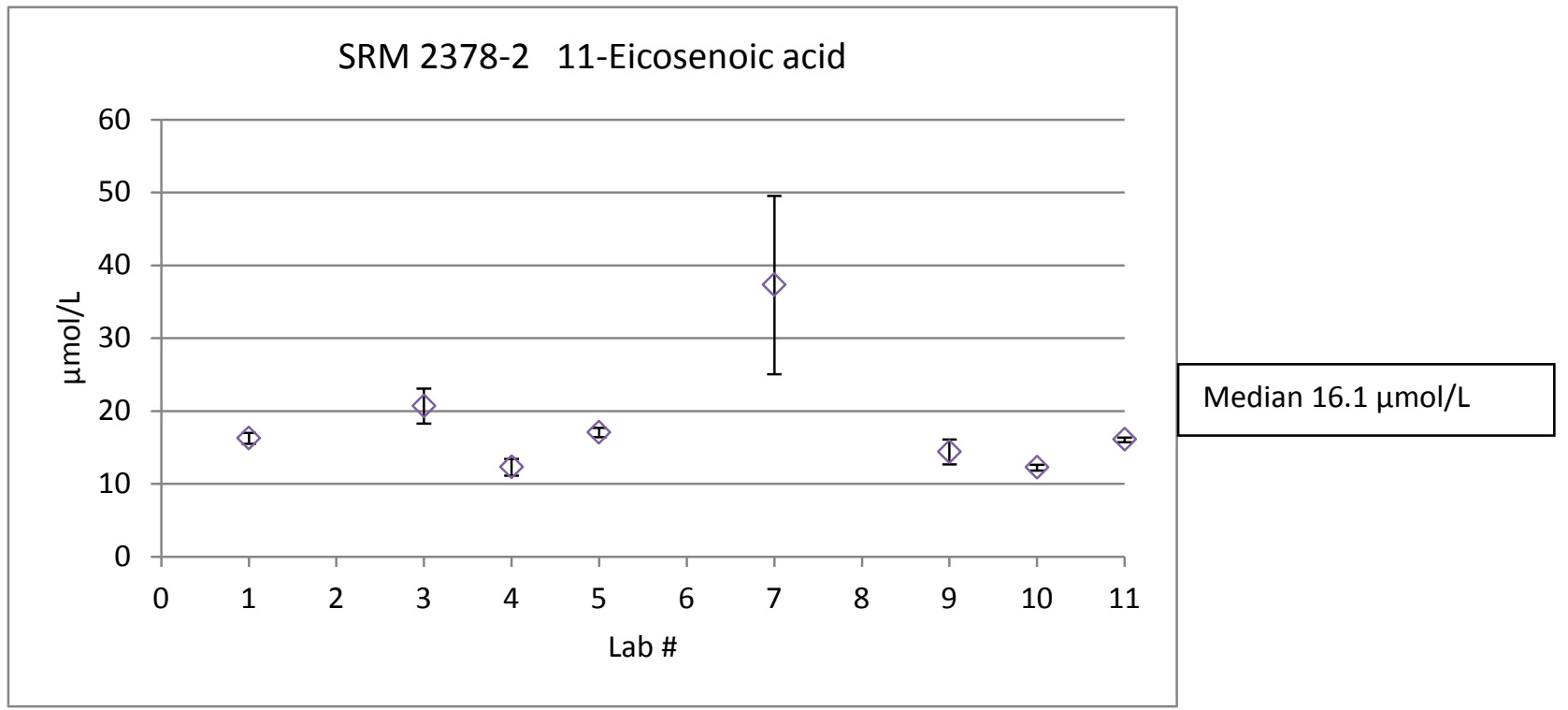



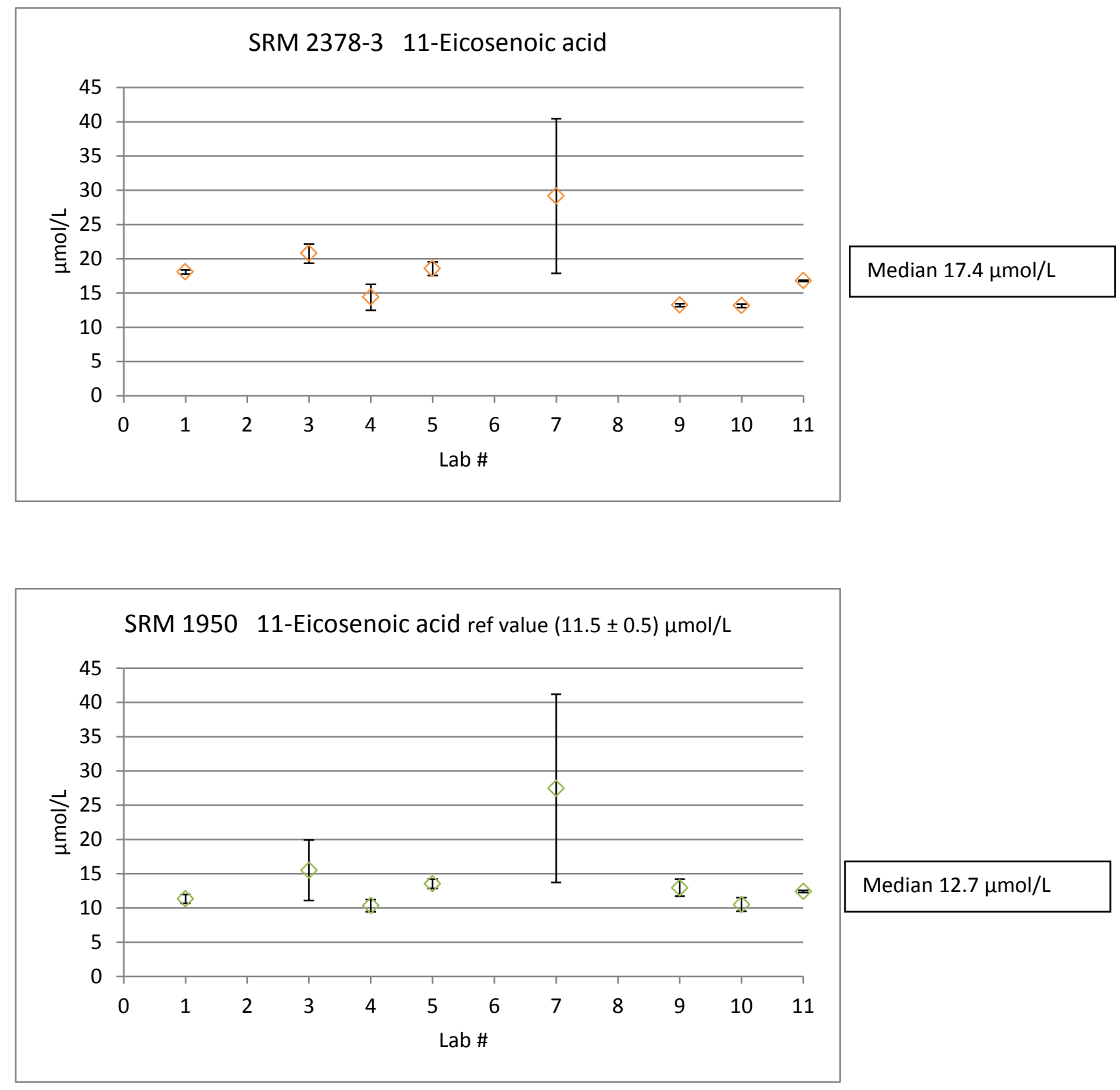

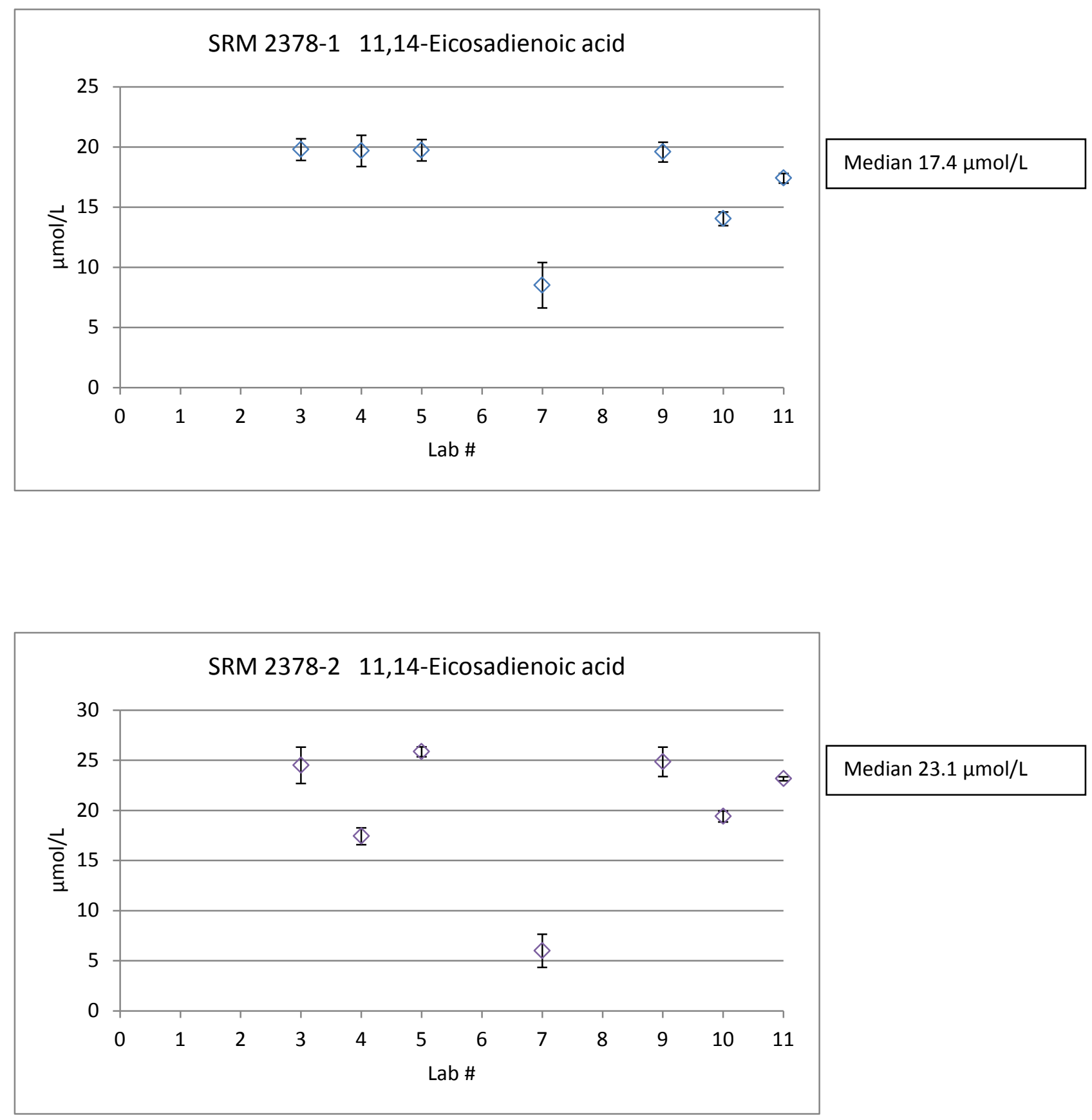

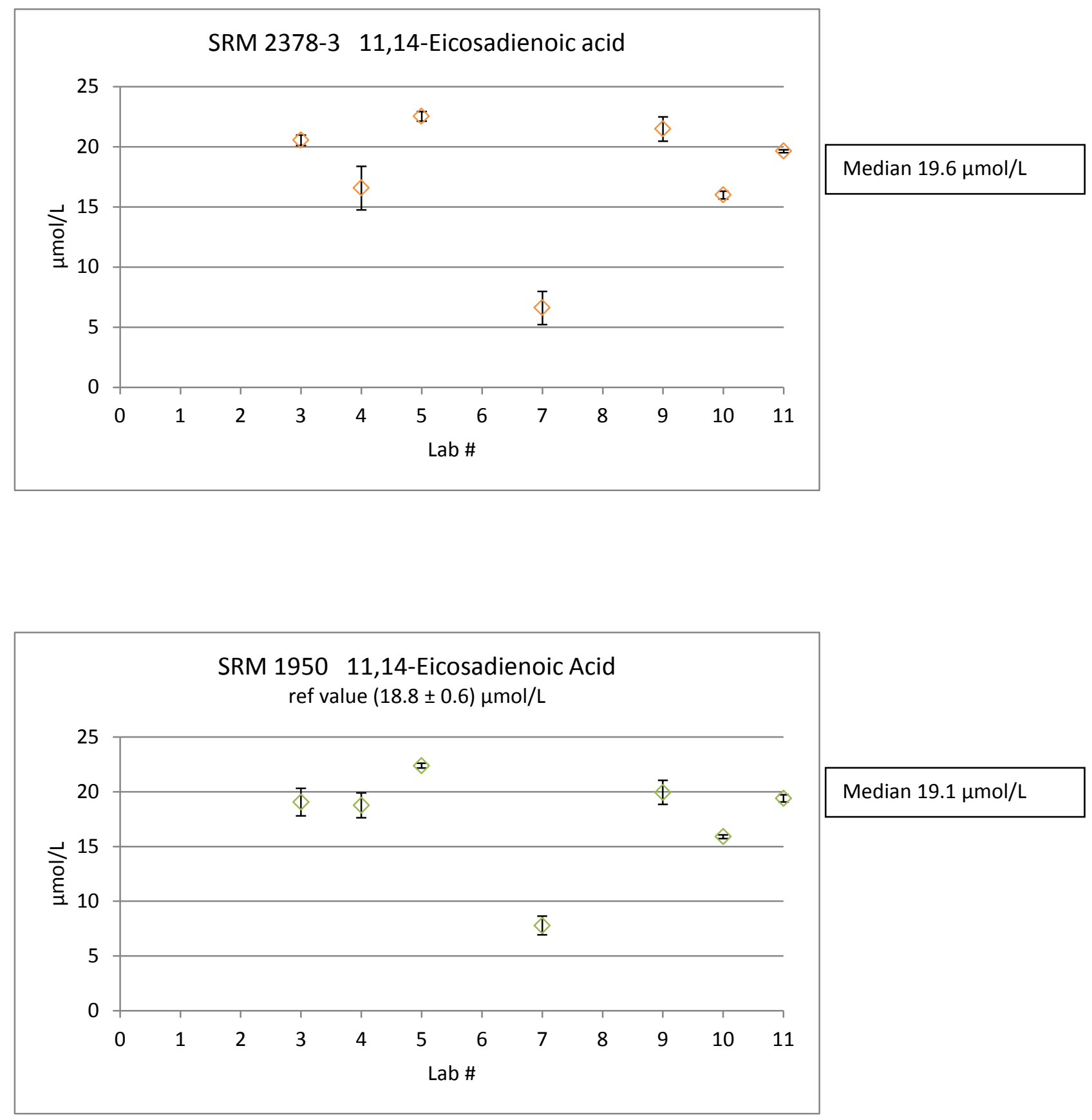


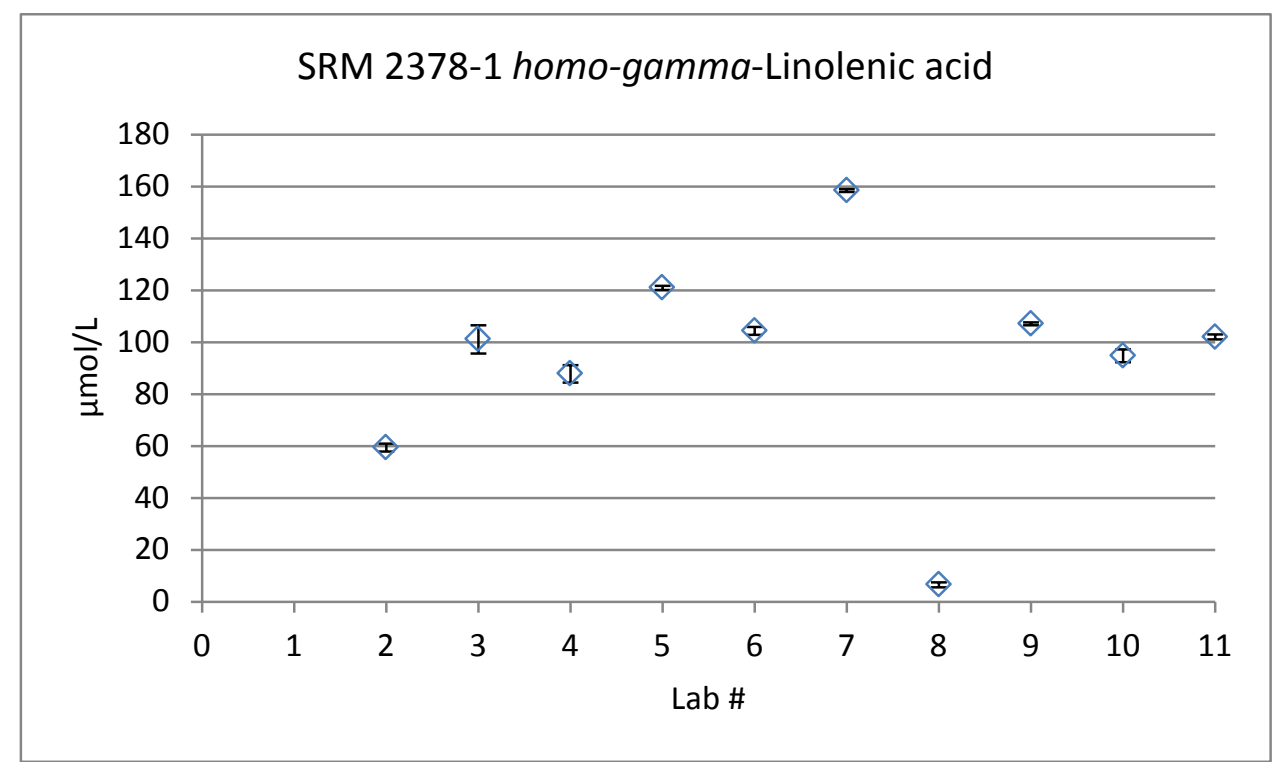

Median (excluding lab 8)

$101 \mu \mathrm{mol} / \mathrm{L}$

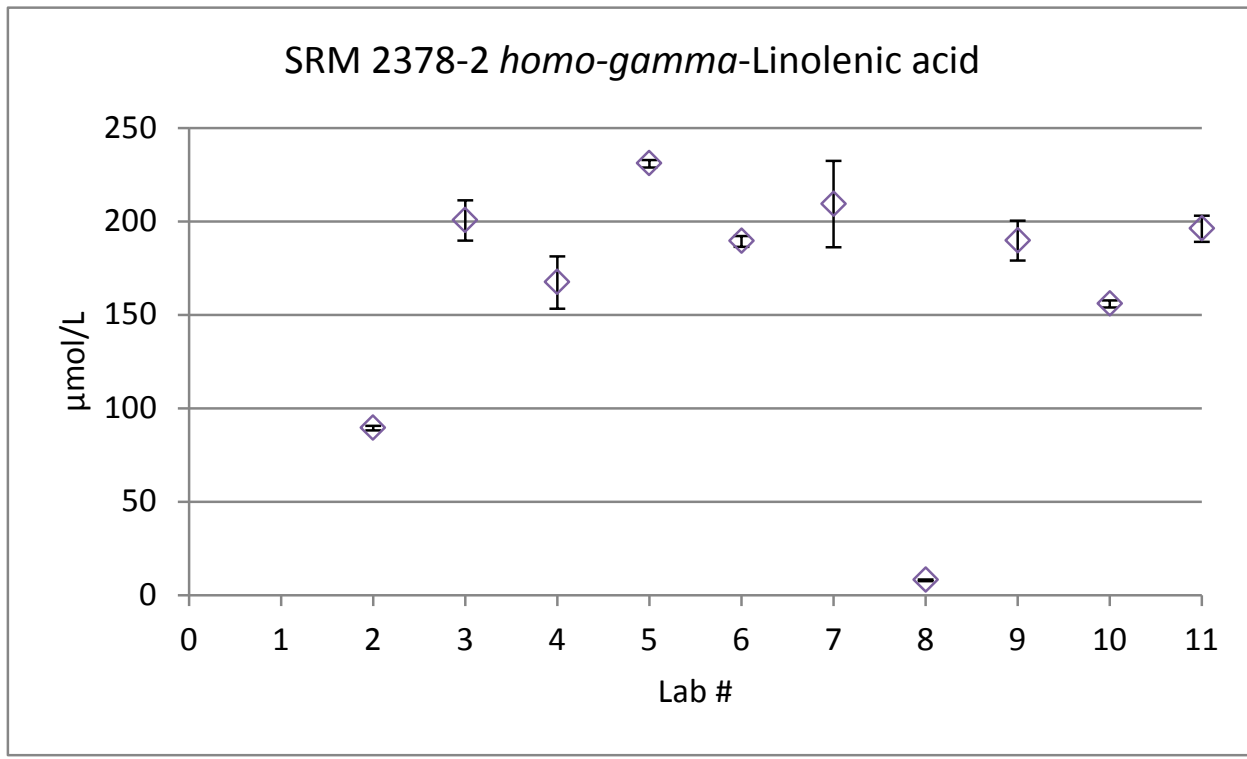

Median (excluding lab 8) $190 \mu \mathrm{mol} / \mathrm{L}$ 

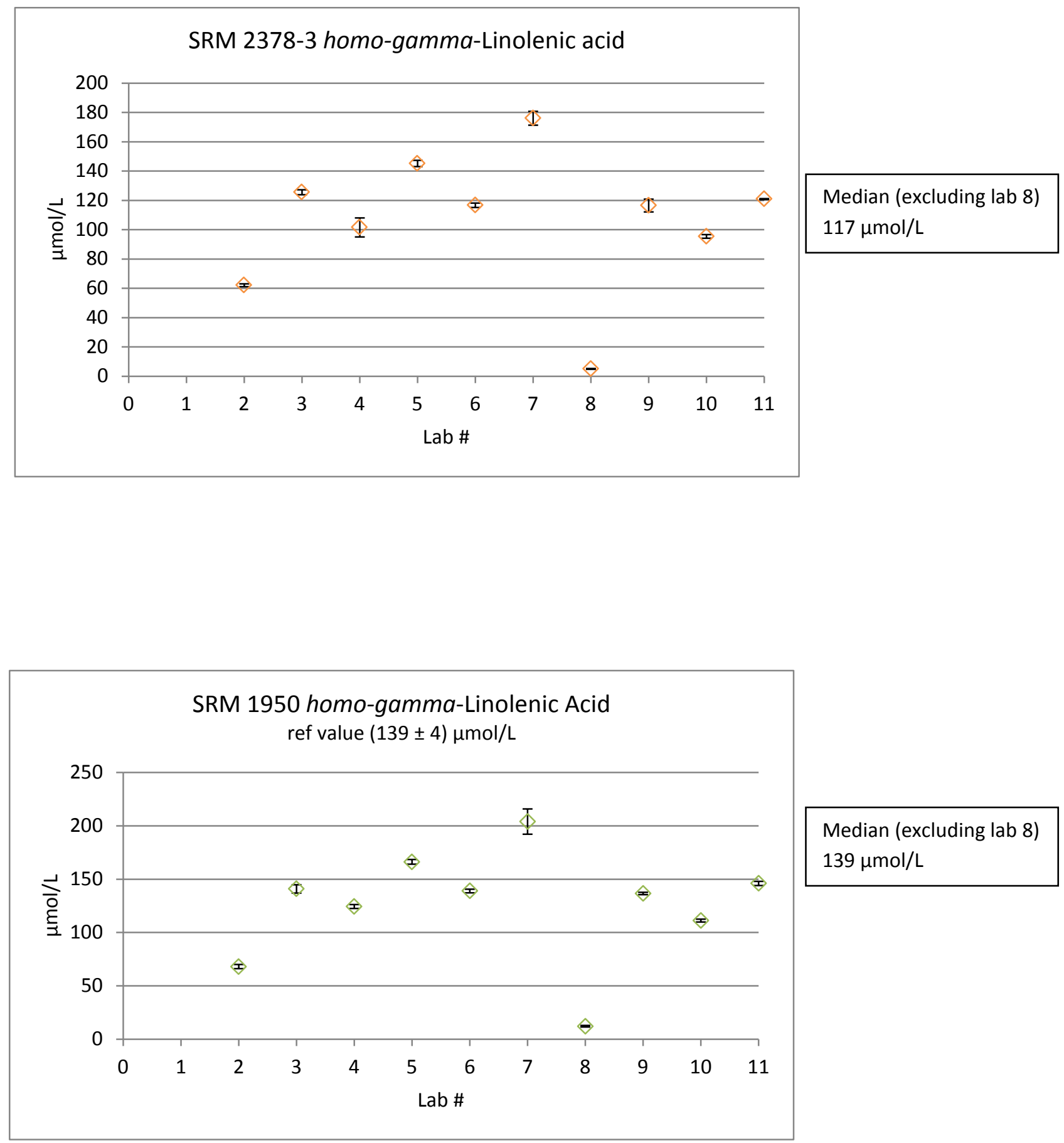

Median (excluding lab 8) $139 \mu \mathrm{mol} / \mathrm{L}$ 


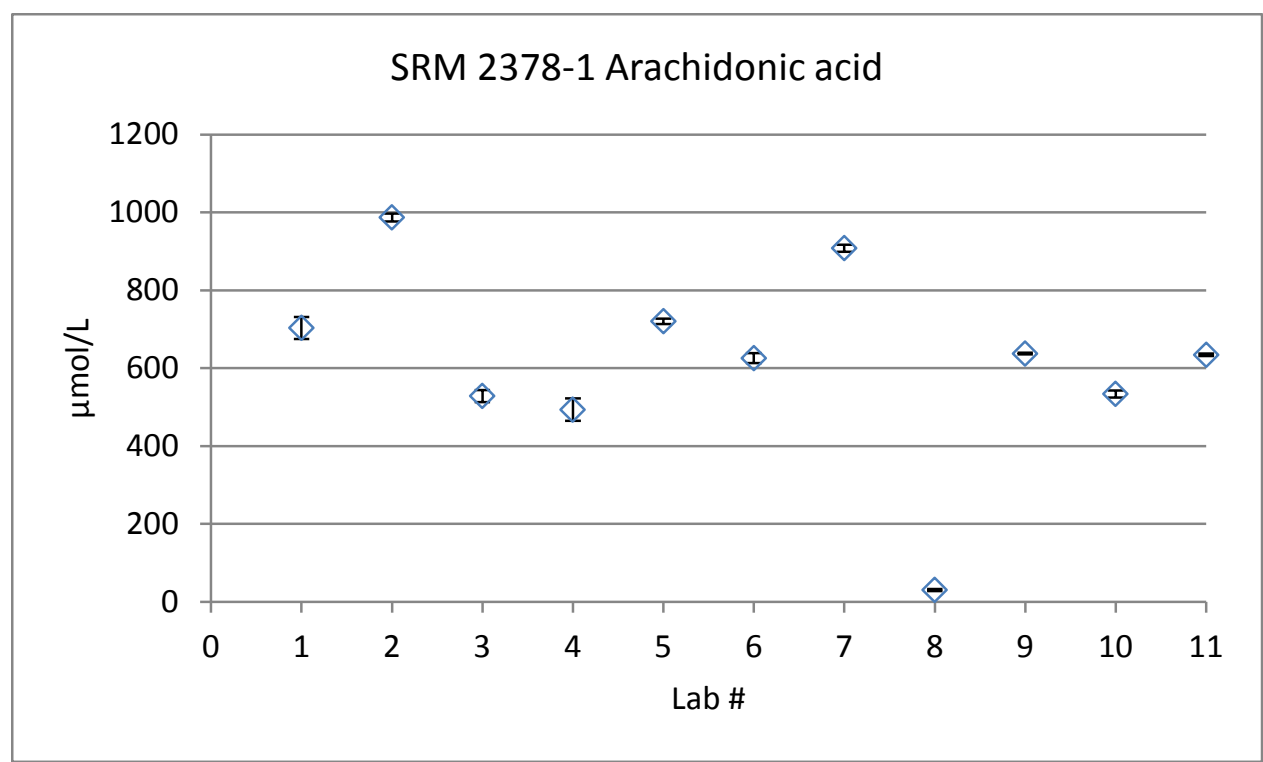

Median (excluding lab 8) $630 \mu \mathrm{mol} / \mathrm{L}$

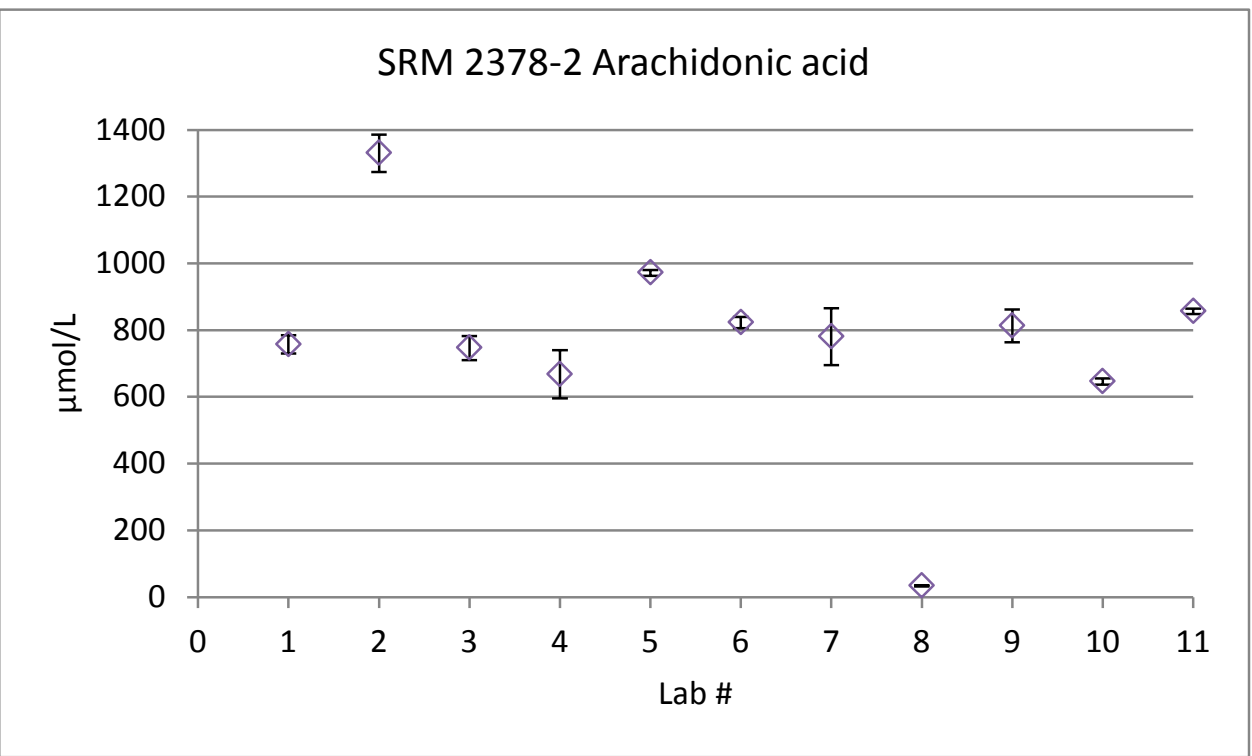

Median (excluding lab 8) $797 \mu \mathrm{mol} / \mathrm{L}$ 

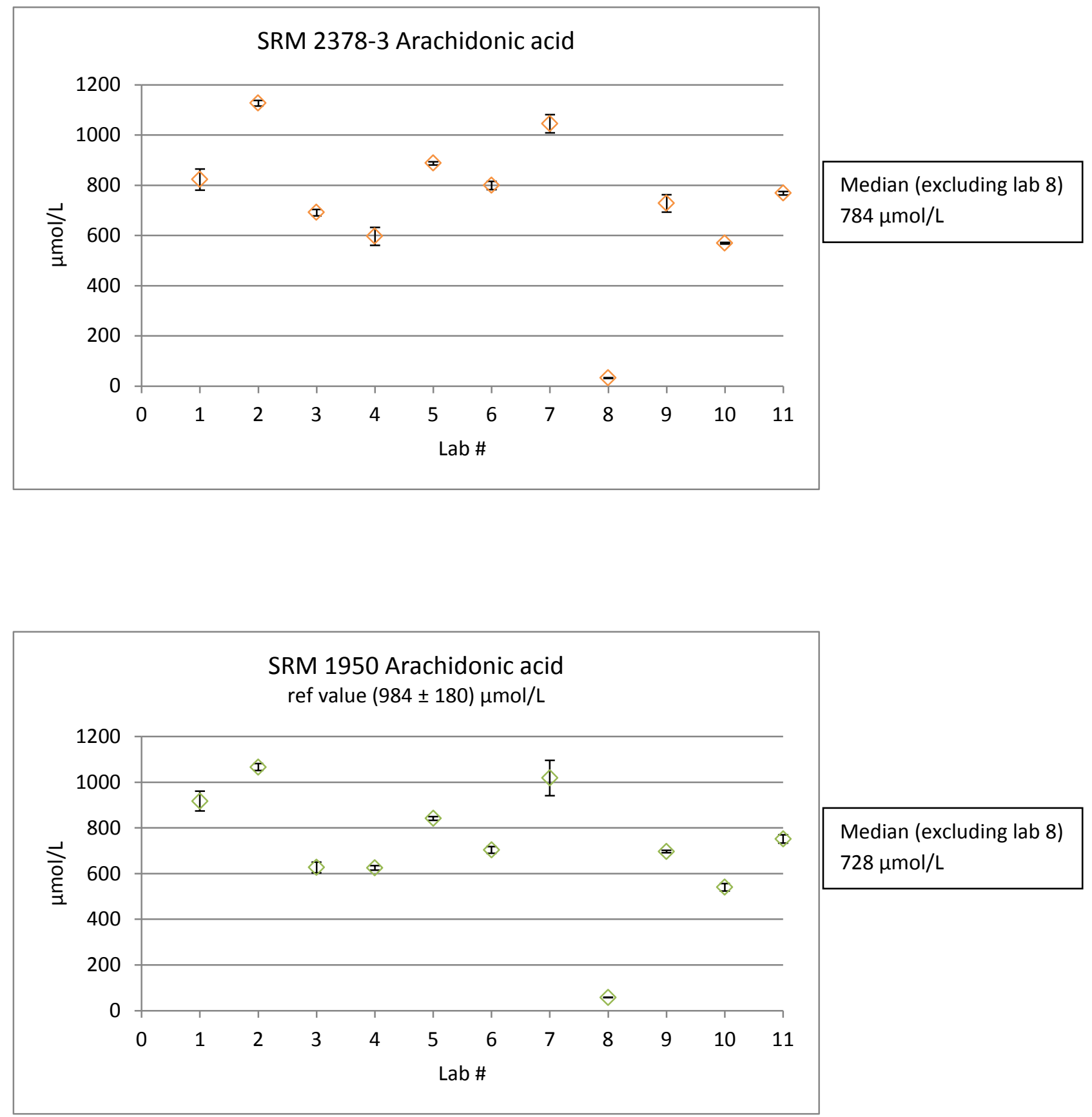


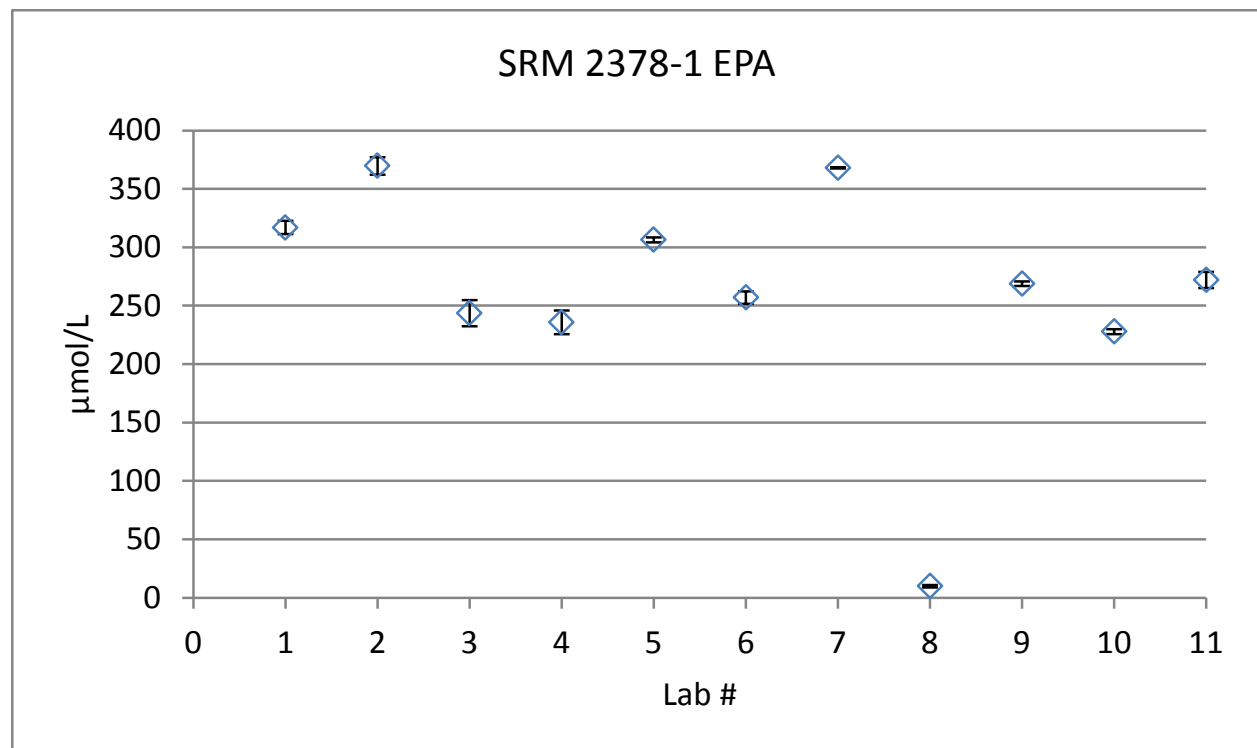

Median (excluding lab 8)

$263 \mu \mathrm{mol} / \mathrm{L}$

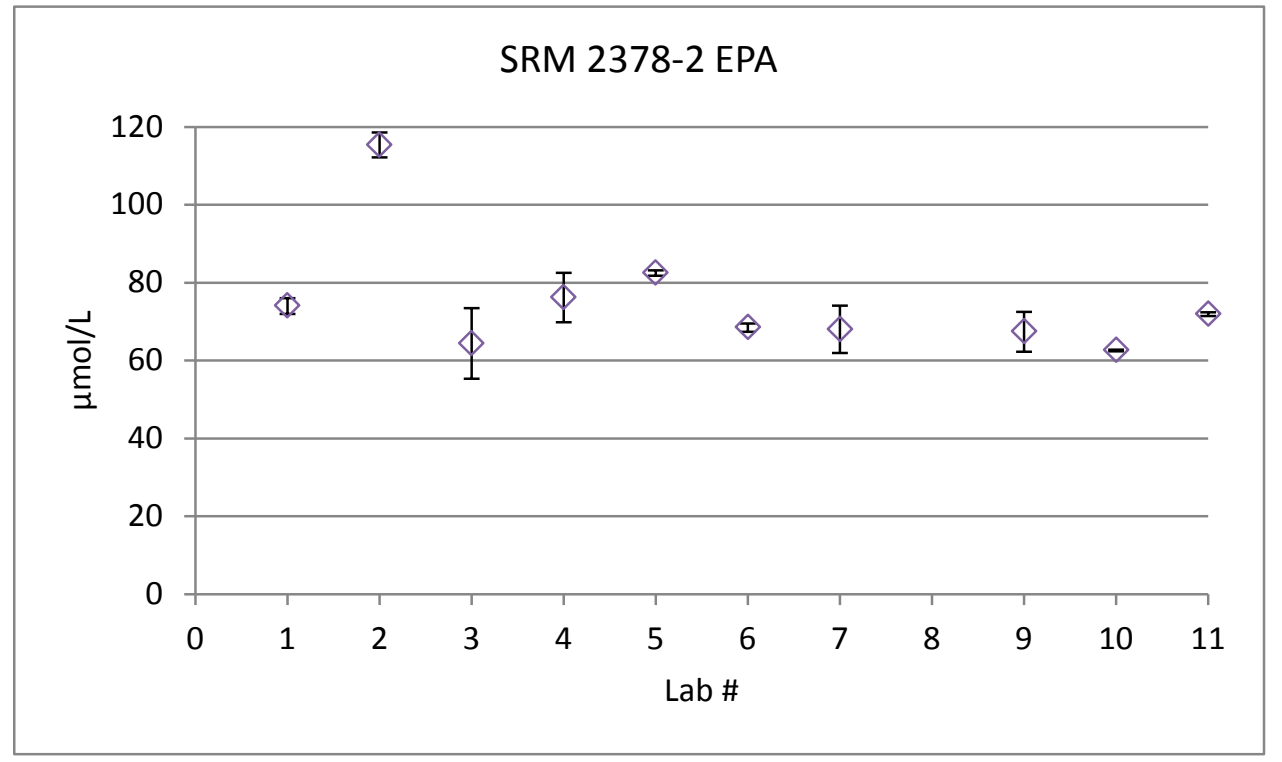

Median $70.2 \mu \mathrm{mol} / \mathrm{L}$ 


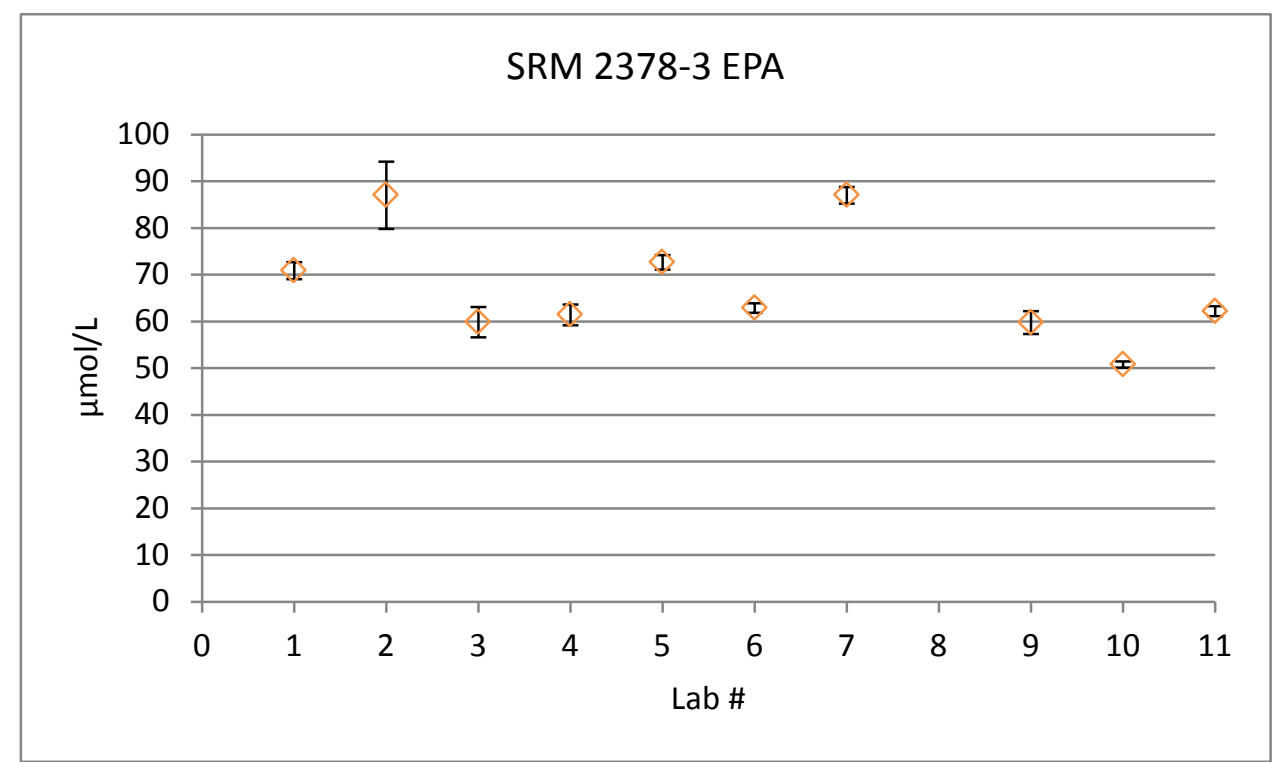

Median $62.5 \mu \mathrm{mol} / \mathrm{L}$

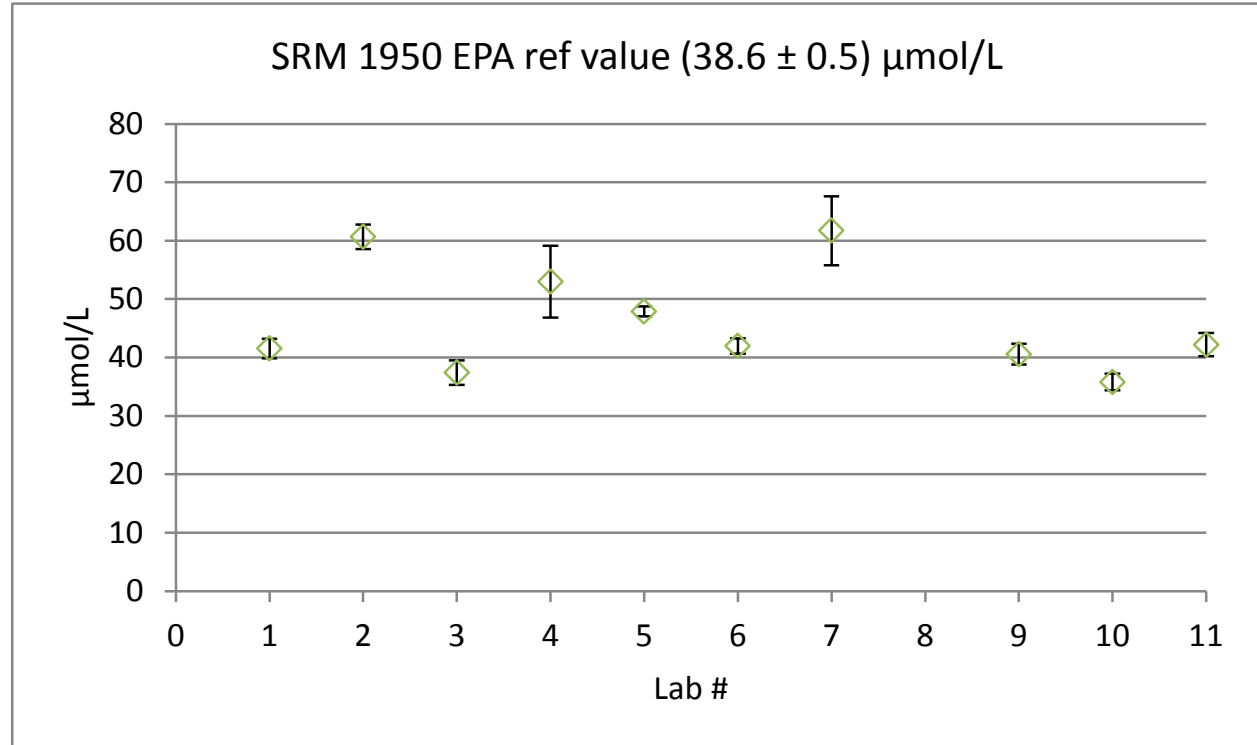

Median $42.1 \mu \mathrm{mol} / \mathrm{L}$ 


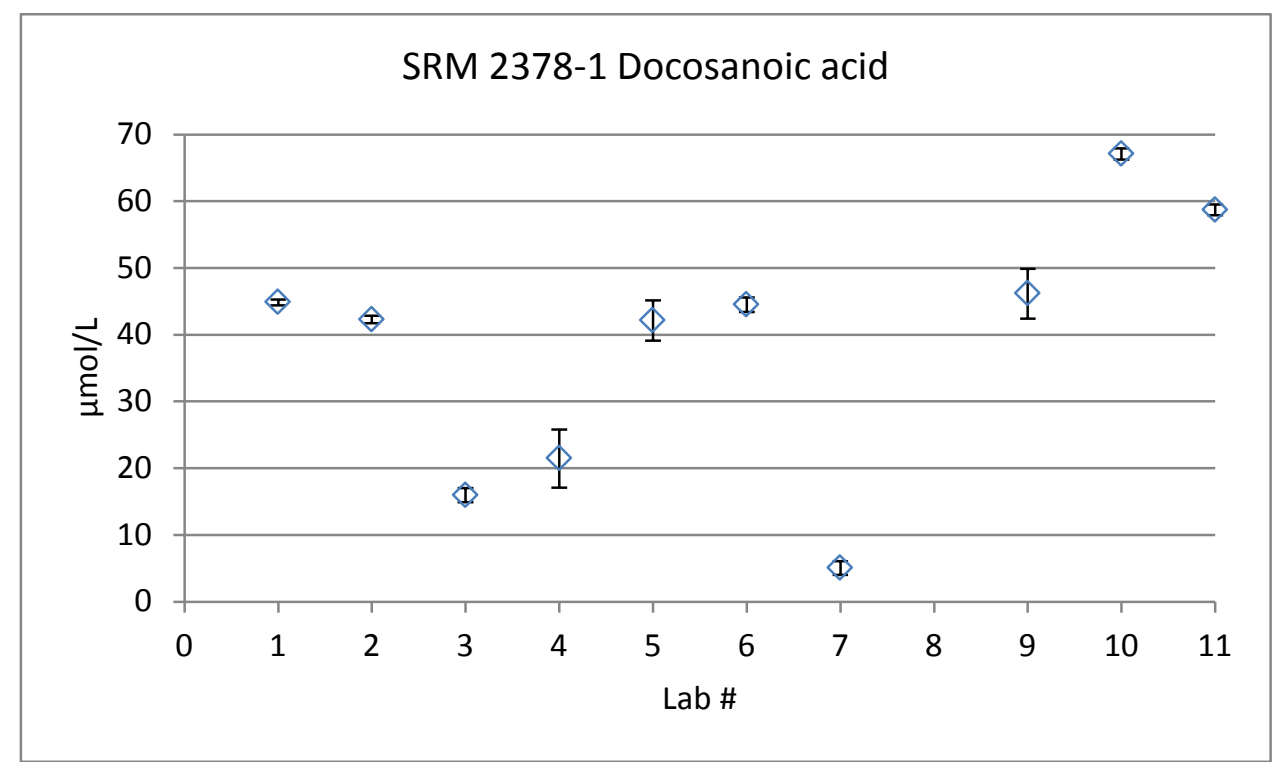

Median $42.2 \mu \mathrm{mol} / \mathrm{L}$

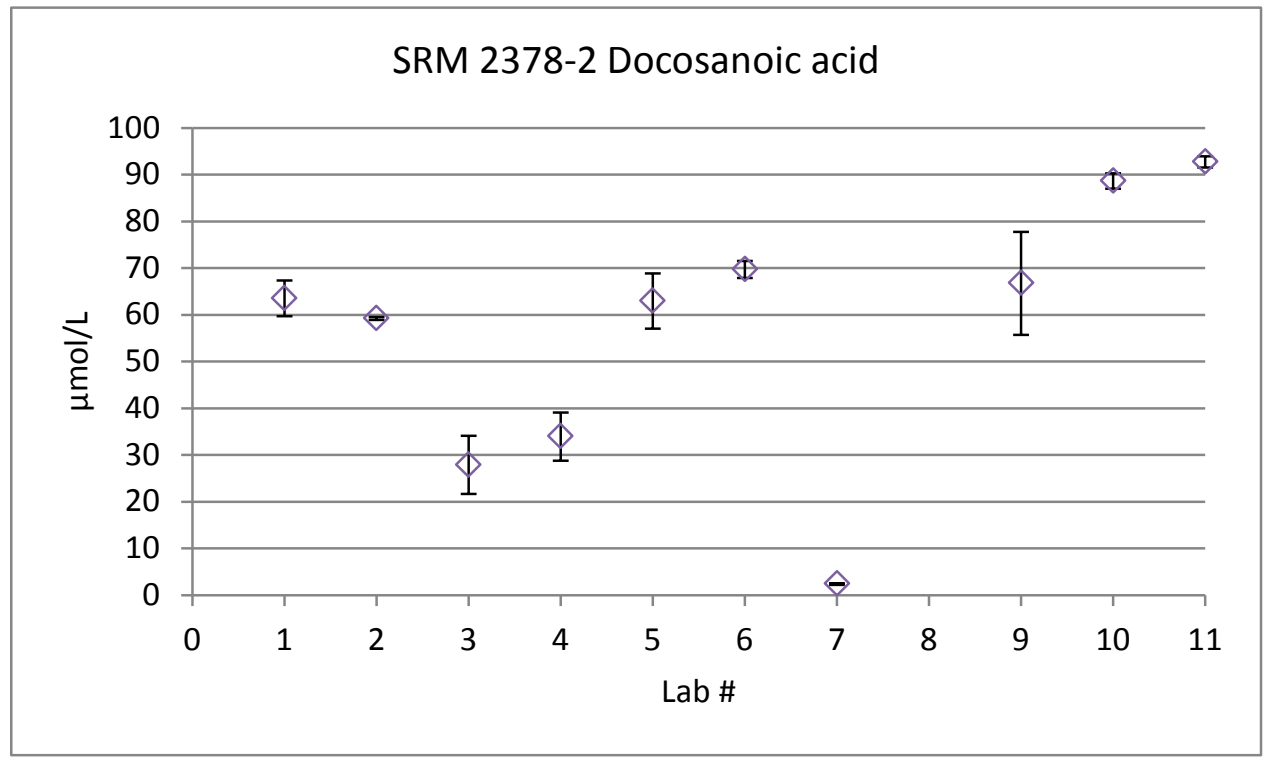

Median $63.2 \mu \mathrm{mol} / \mathrm{L}$ 

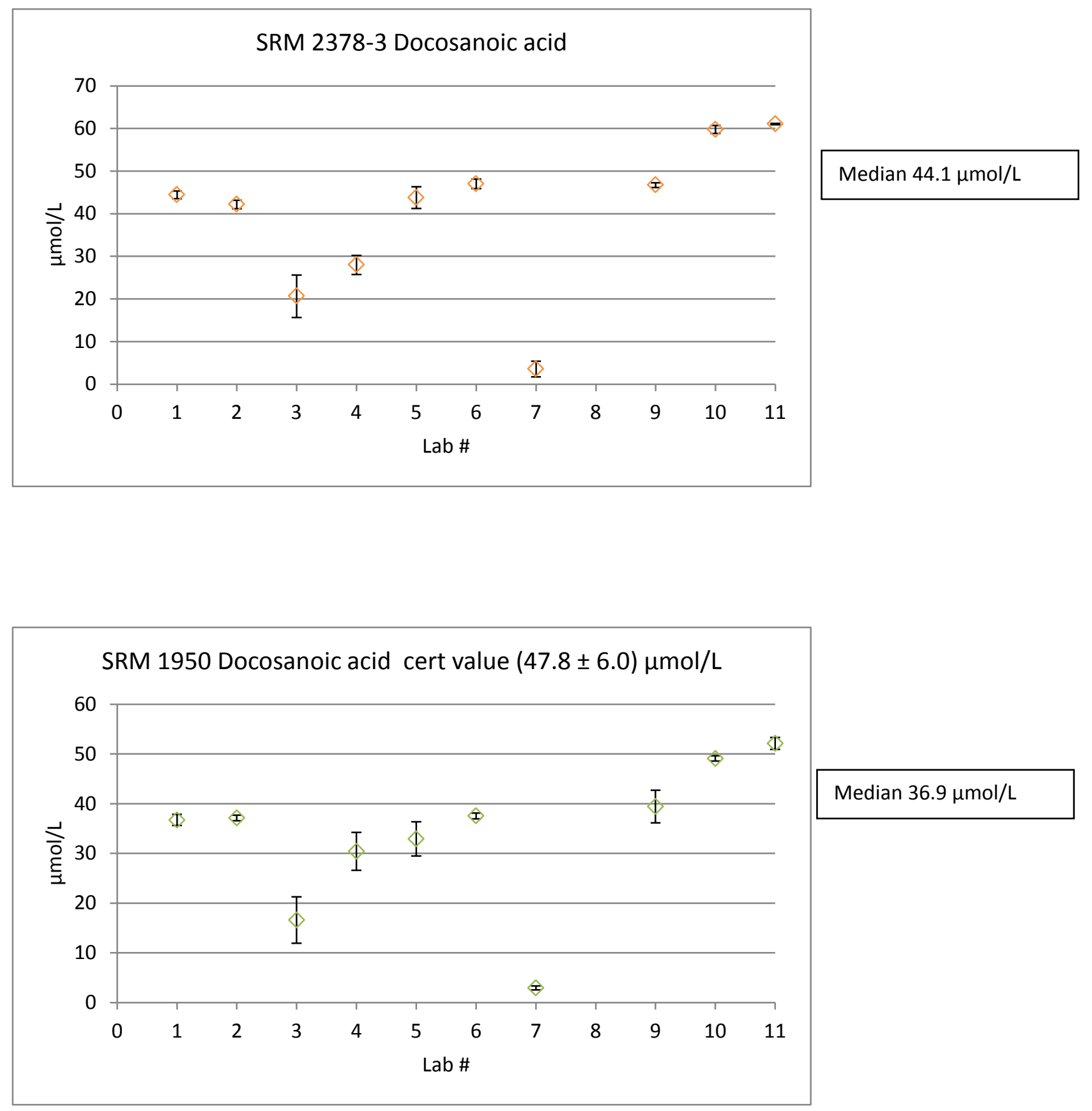


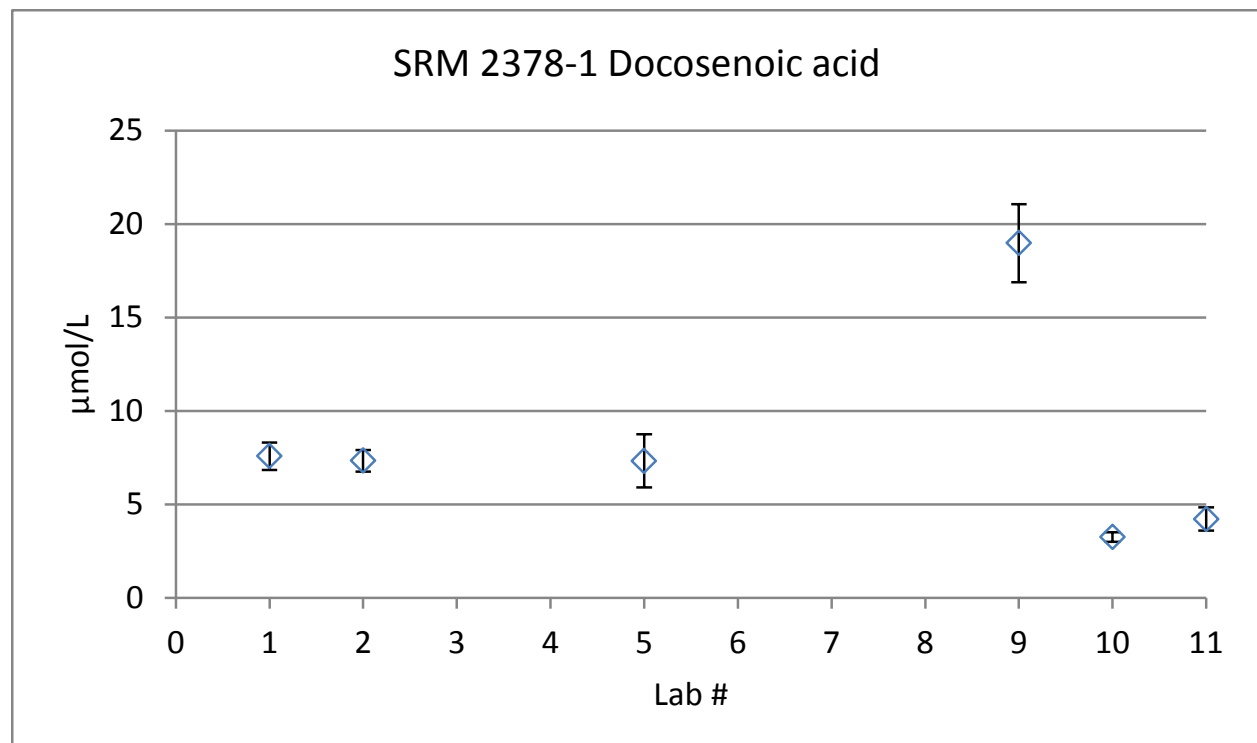

Median $5.76 \mu \mathrm{mol} / \mathrm{L}$

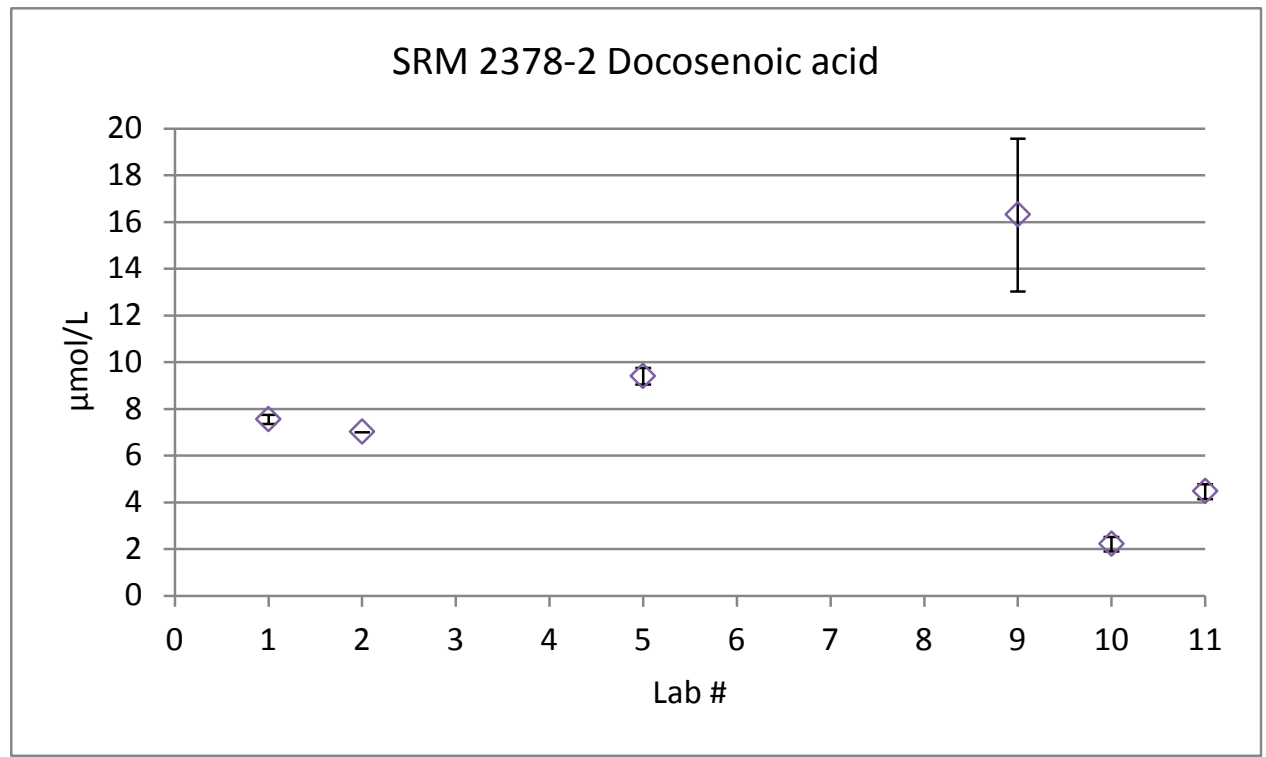

Median $7.27 \mu \mathrm{mol} / \mathrm{L}$ 

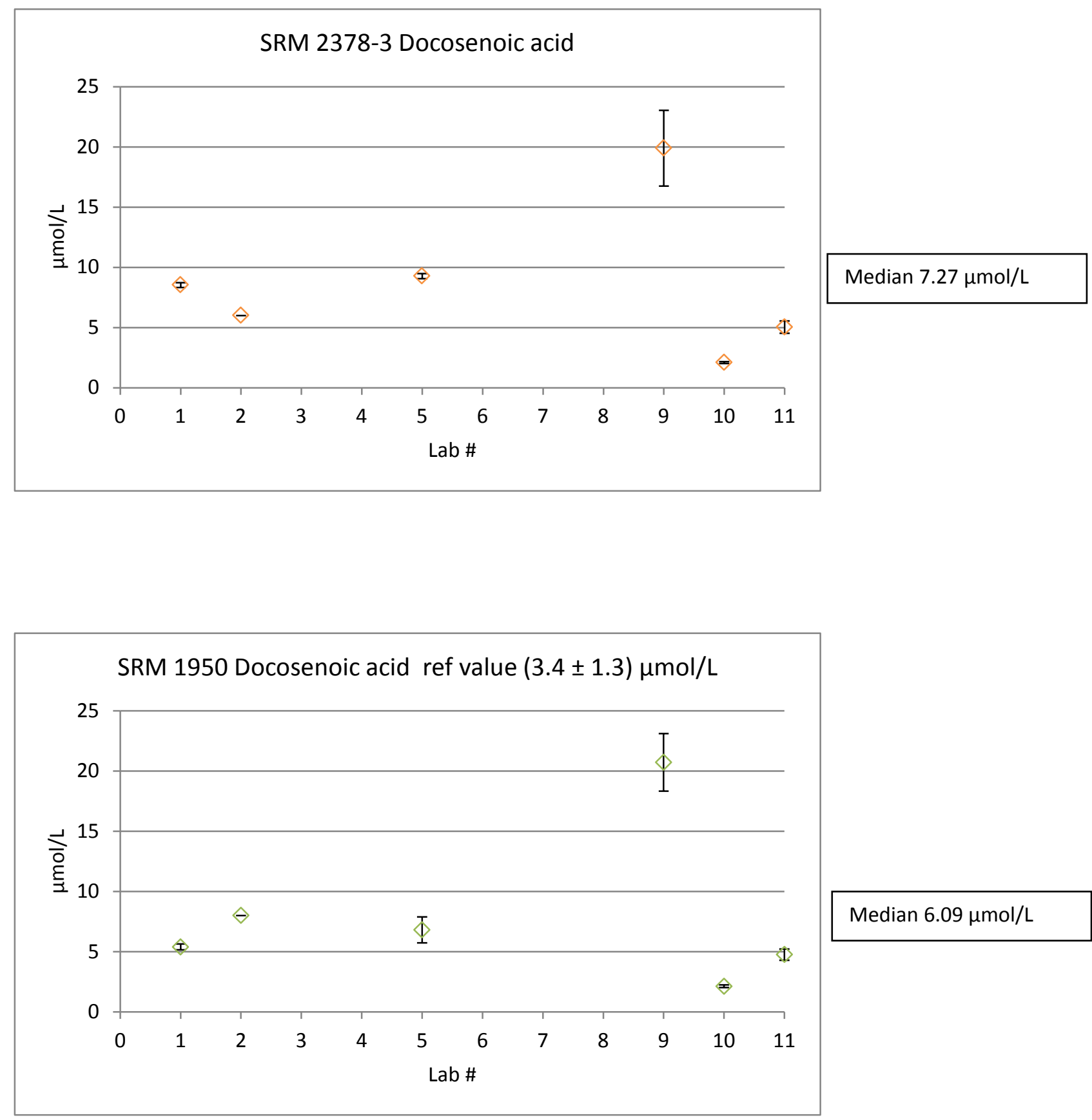

Median $6.09 \mu \mathrm{mol} / \mathrm{L}$ 


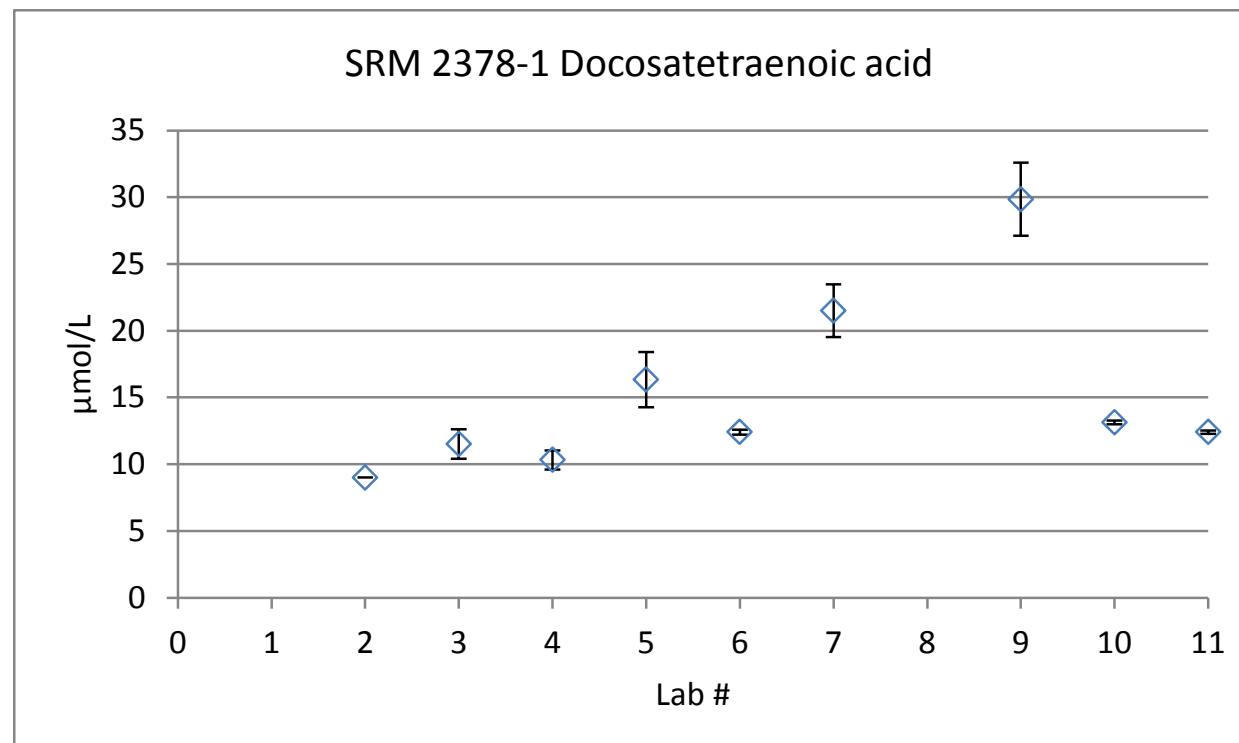

Median $12.4 \mu \mathrm{mol} / \mathrm{L}$

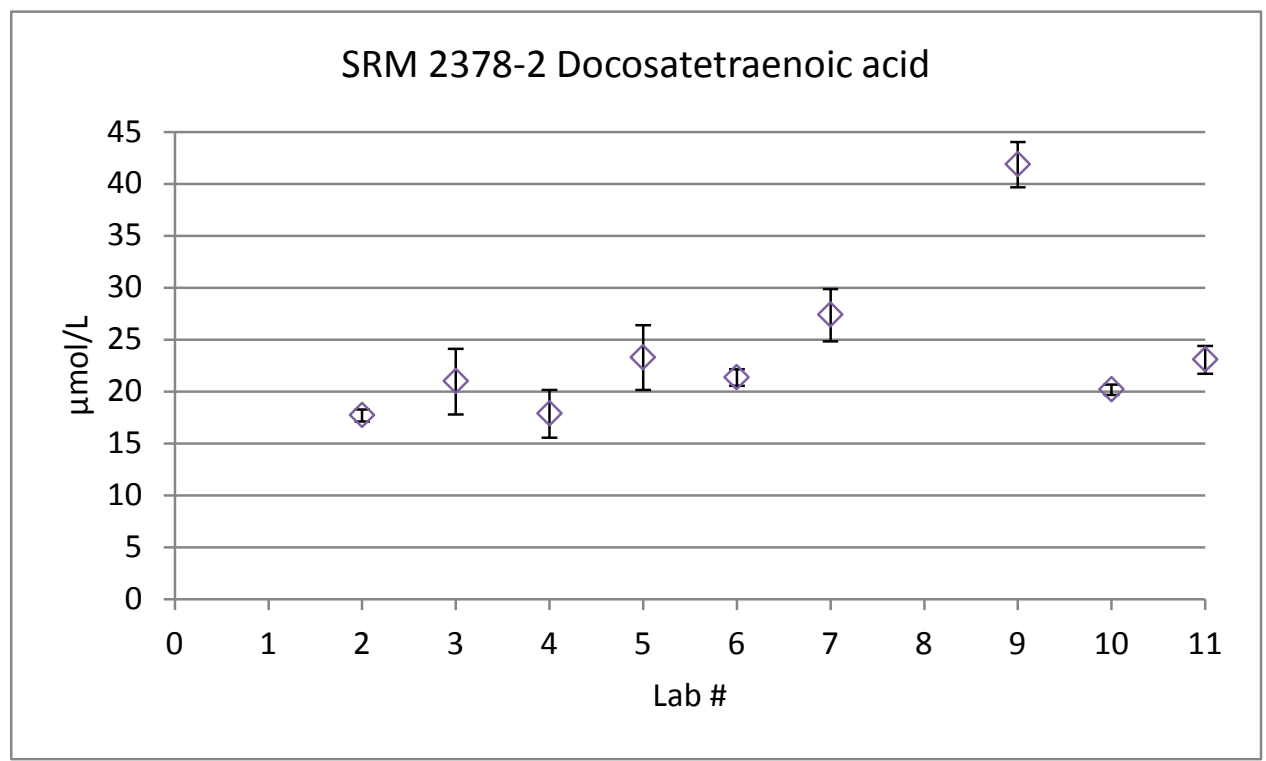

Median $21.3 \mu \mathrm{mol} / \mathrm{L}$ 

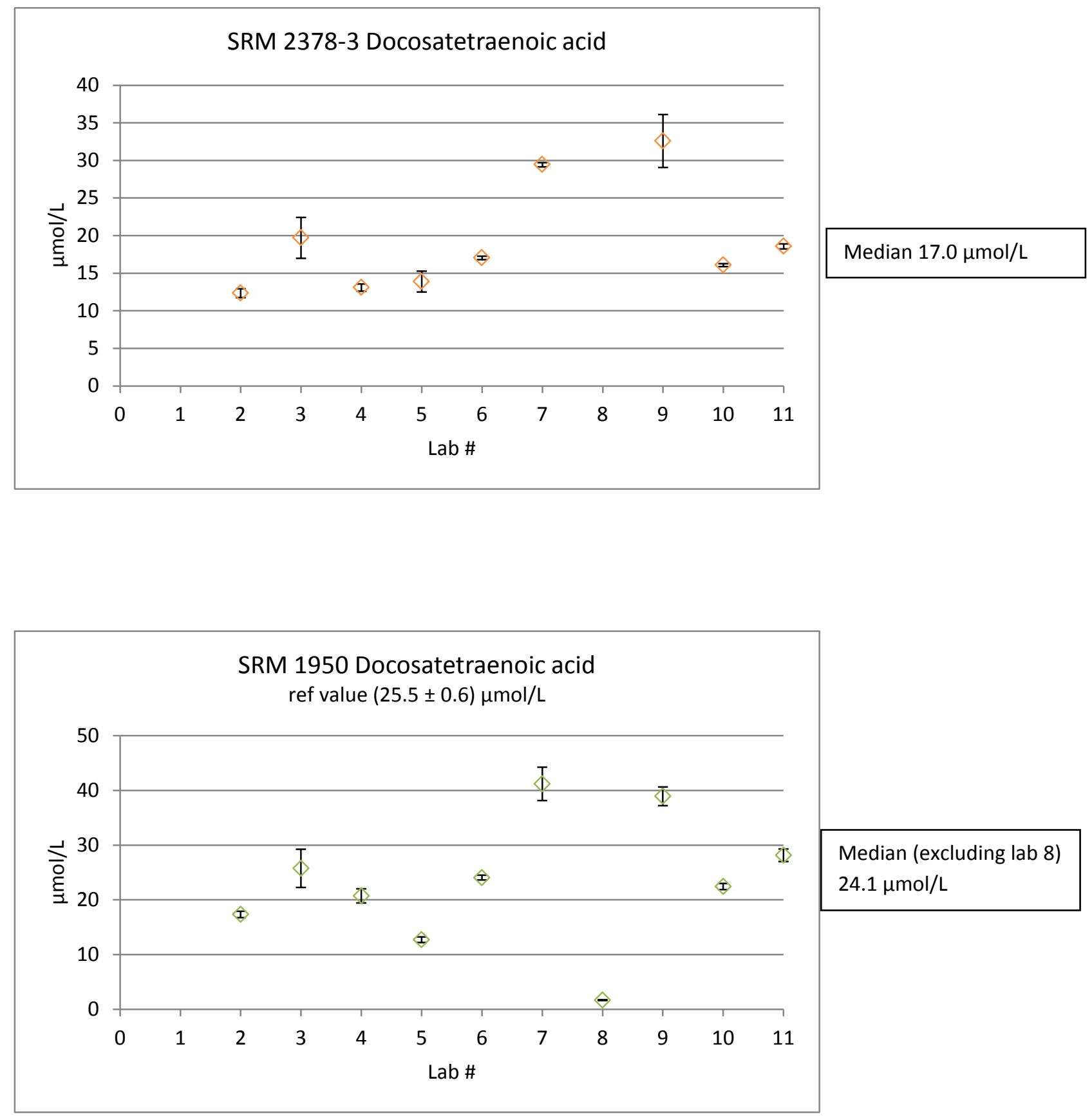


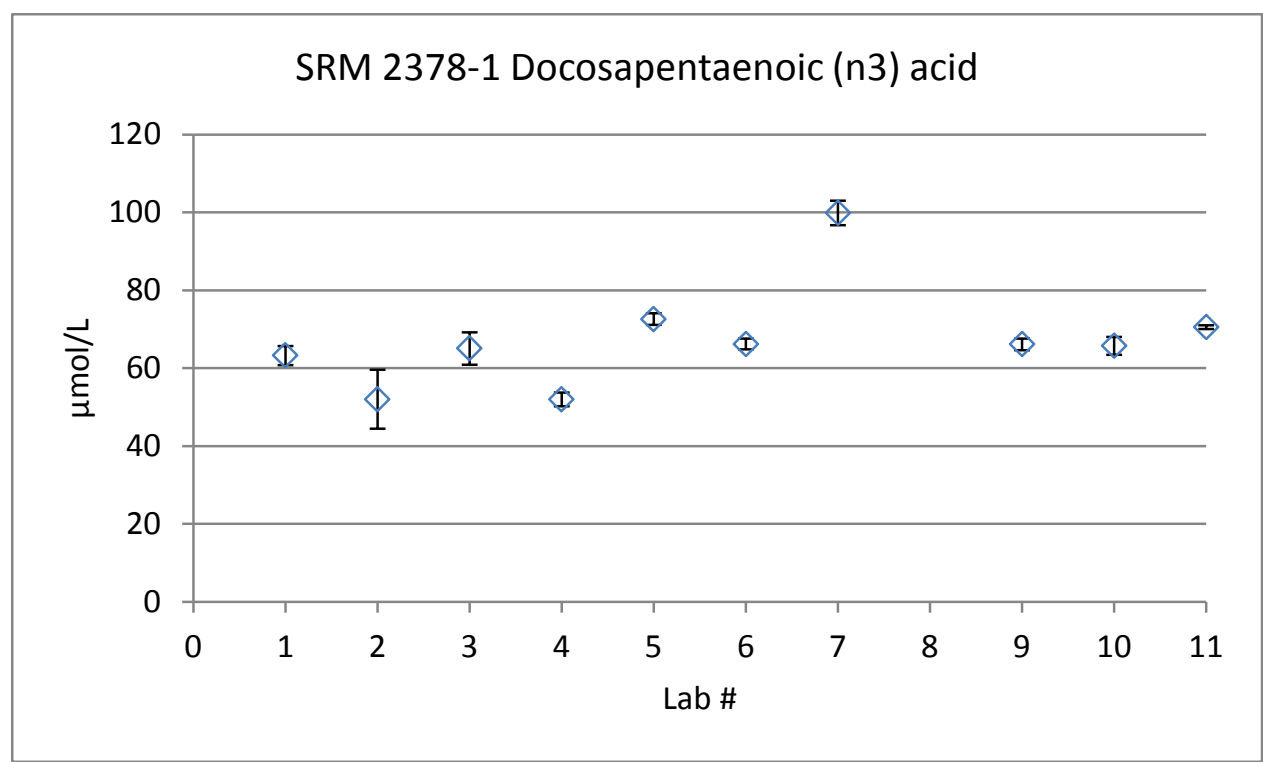

Median $65.4 \mu \mathrm{mol} / \mathrm{L}$

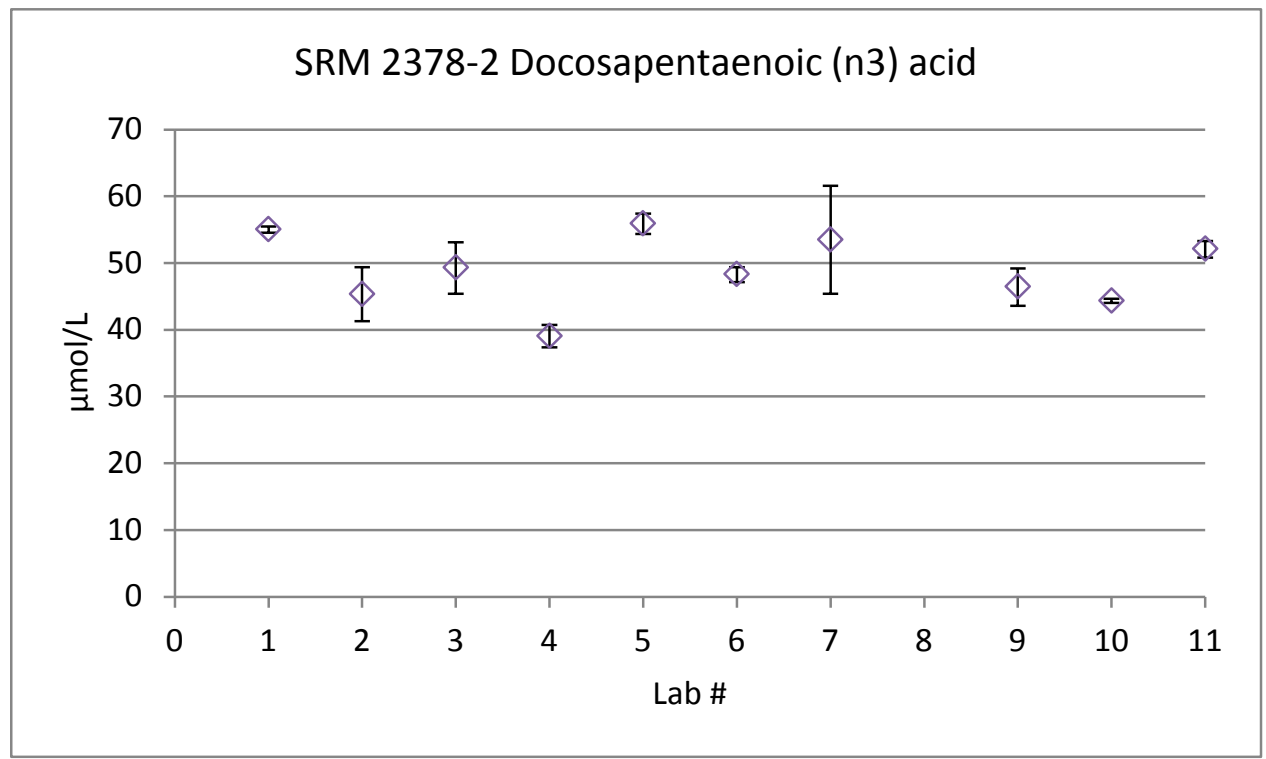

Median $48.8 \mu \mathrm{mol} / \mathrm{L}$ 

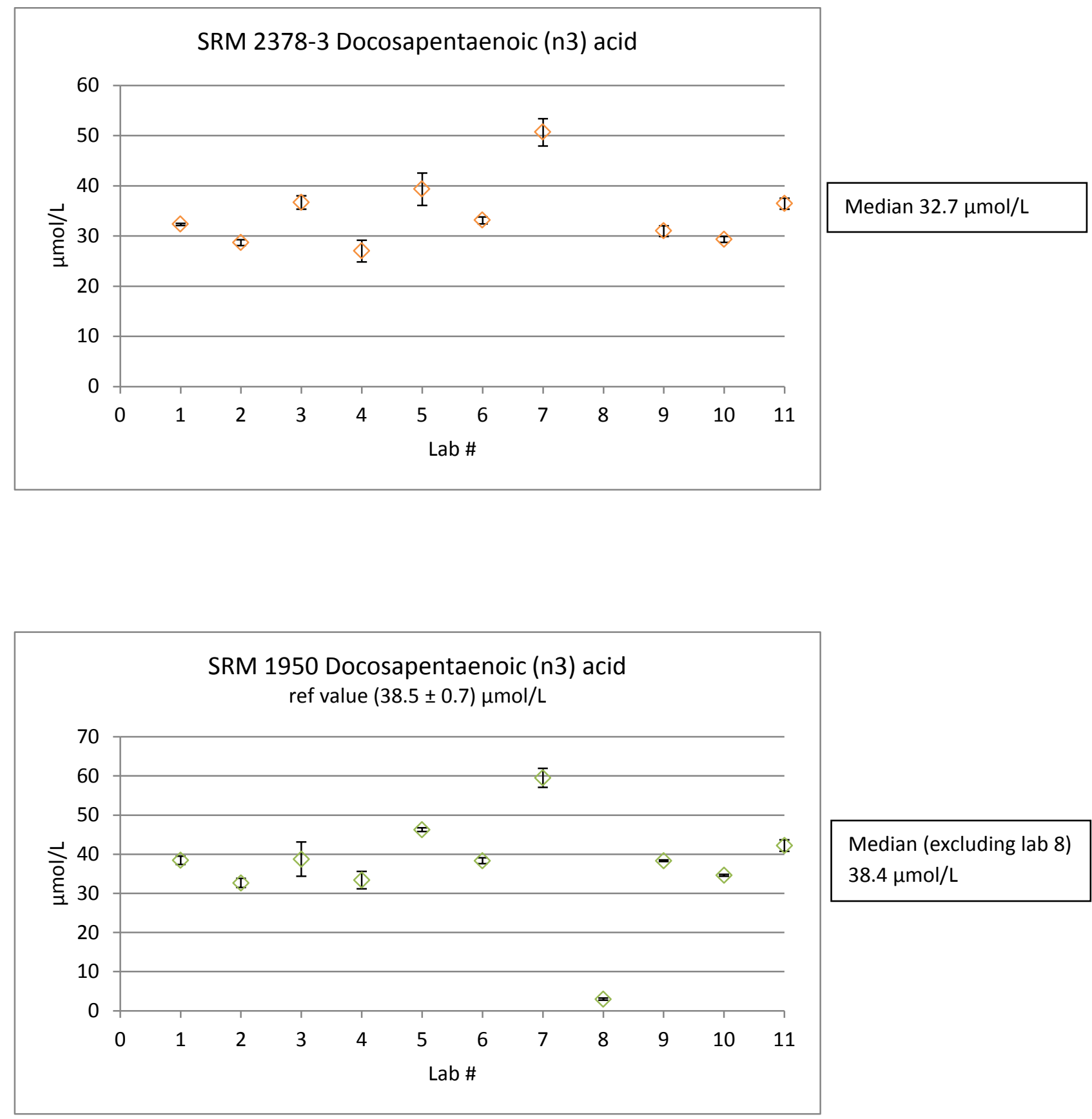

Median (excluding lab 8) $38.4 \mu \mathrm{mol} / \mathrm{L}$ 


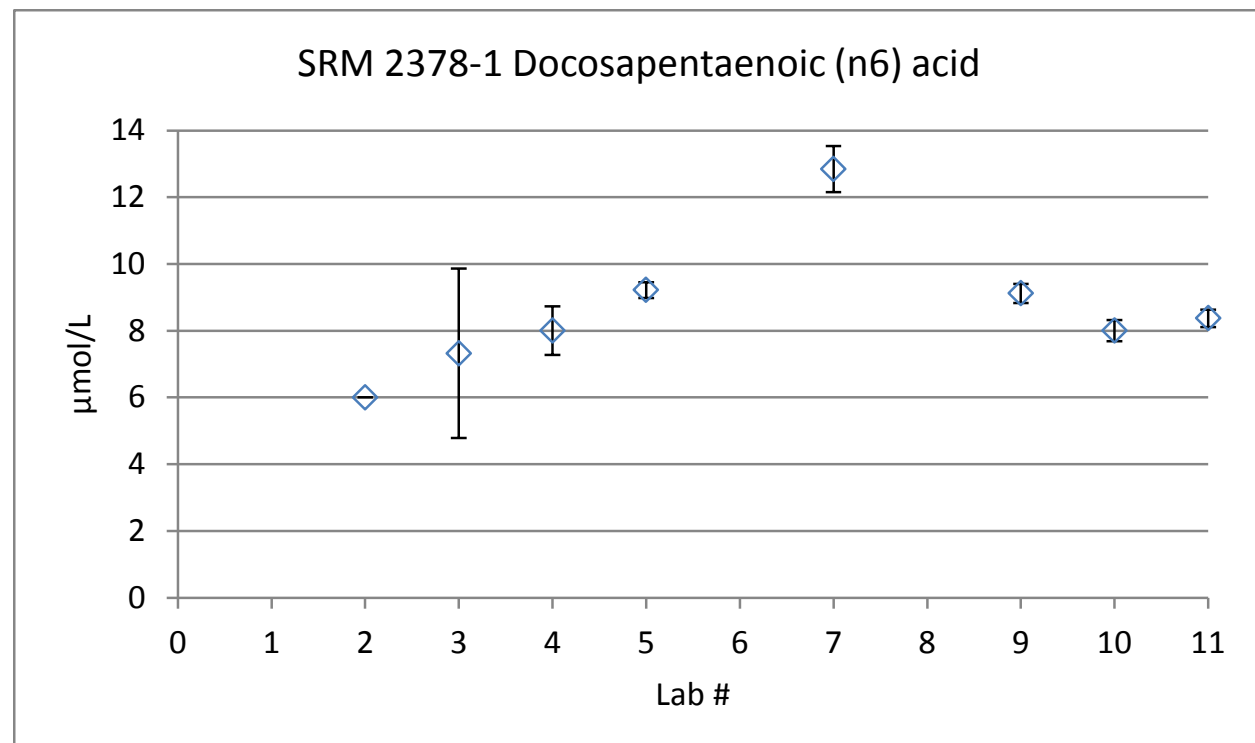

Median $8.00 \mu \mathrm{mol} / \mathrm{L}$

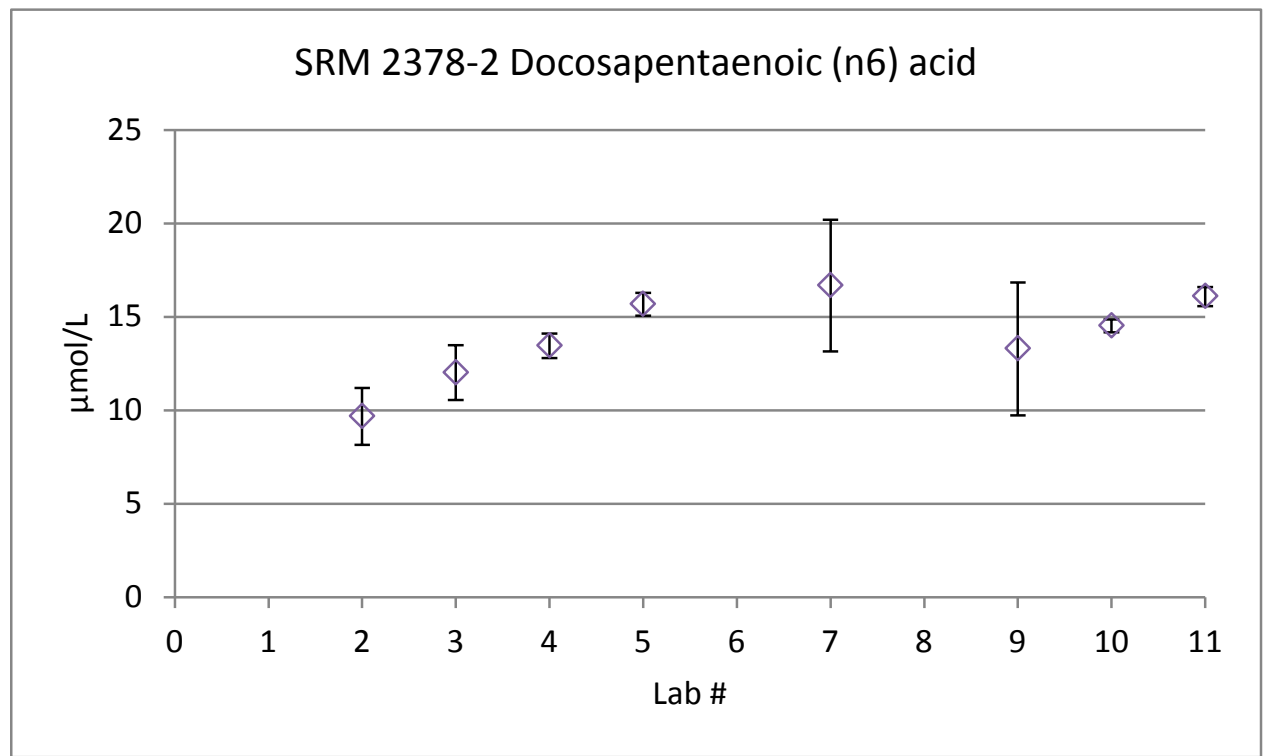

Median $14.0 \mu \mathrm{mol} / \mathrm{L}$ 


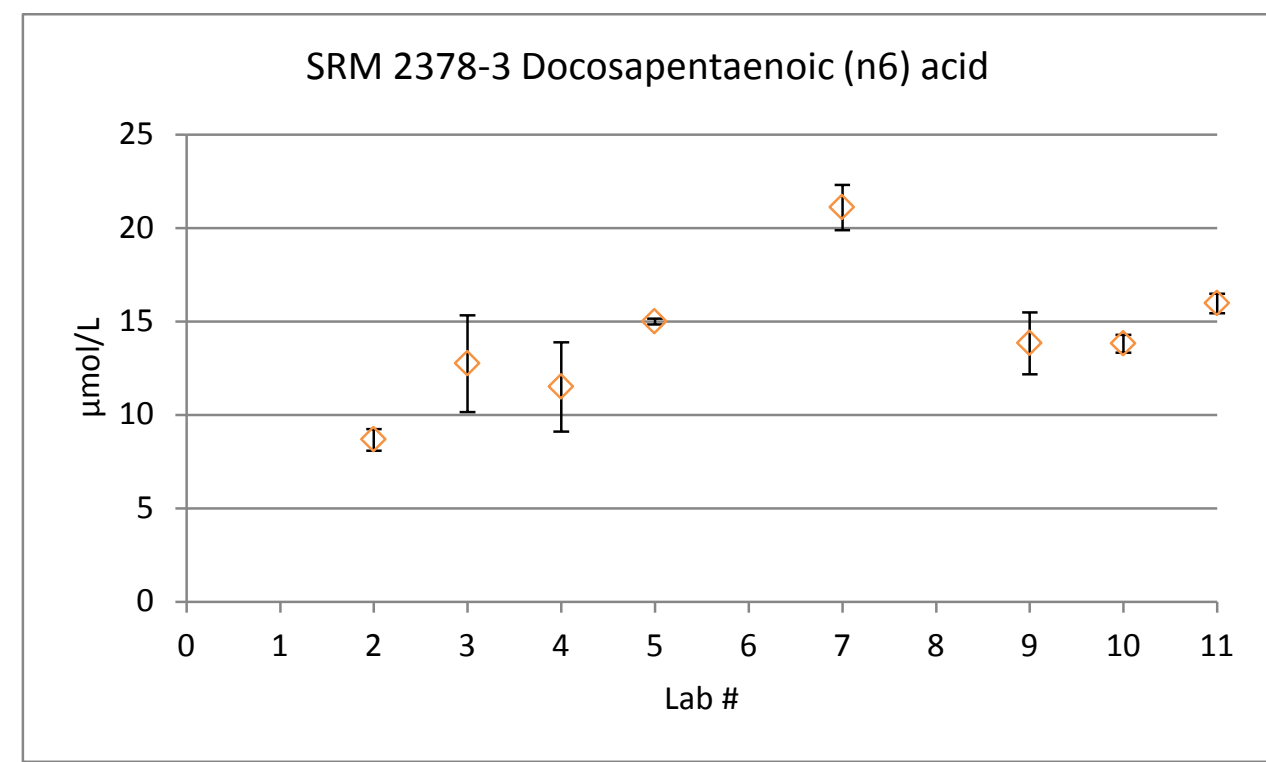

Median $13.8 \mu \mathrm{mol} / \mathrm{L}$

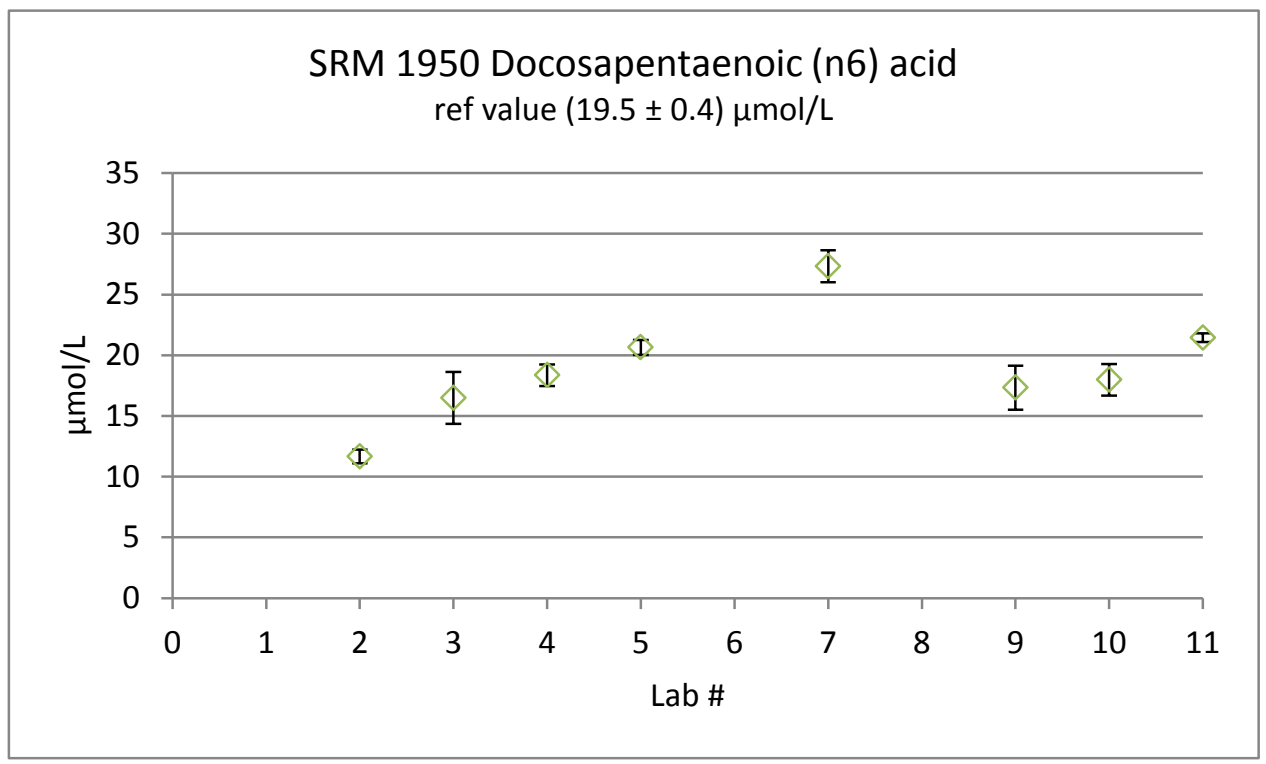

Median $18.2 \mu \mathrm{mol} / \mathrm{L}$ 


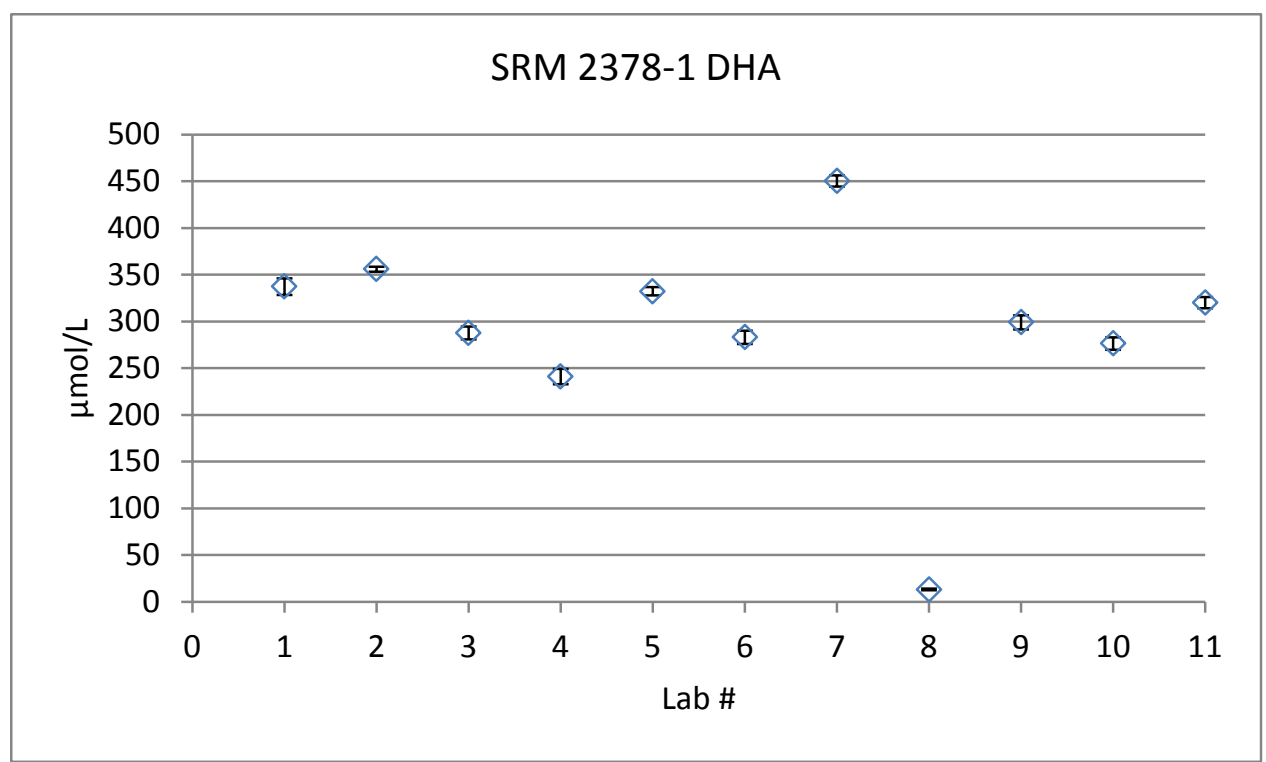

Median (excluding lab 8)

$293 \mu \mathrm{mol} / \mathrm{L}$

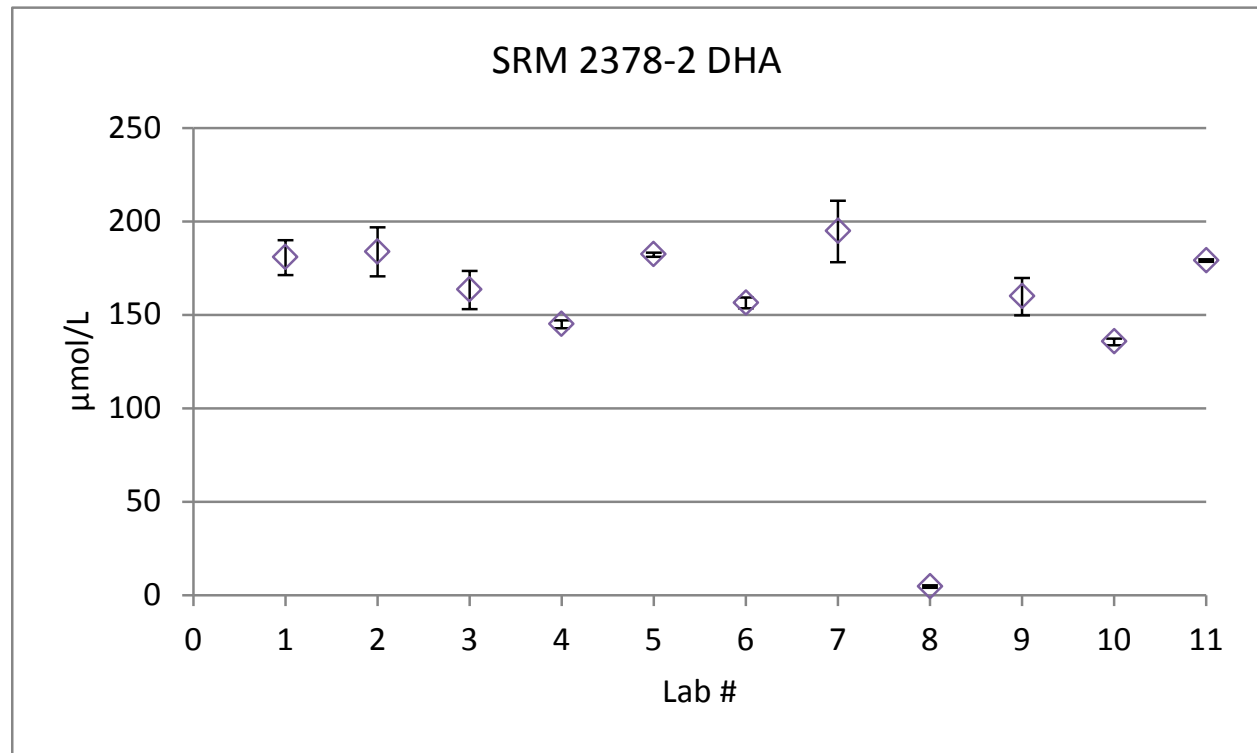

Median (excluding lab 8)

$171 \mu \mathrm{mol} / \mathrm{L}$ 


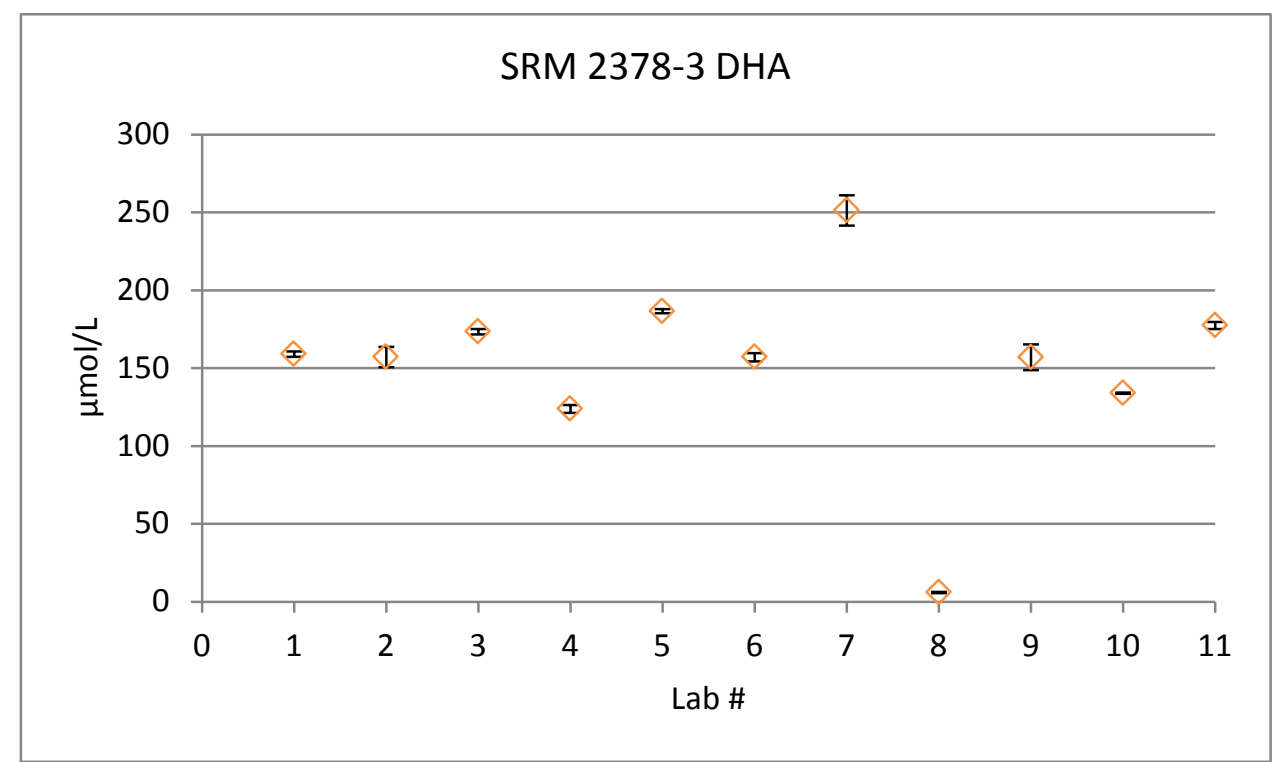

Median (excluding lab 8)

$158 \mu \mathrm{mol} / \mathrm{L}$

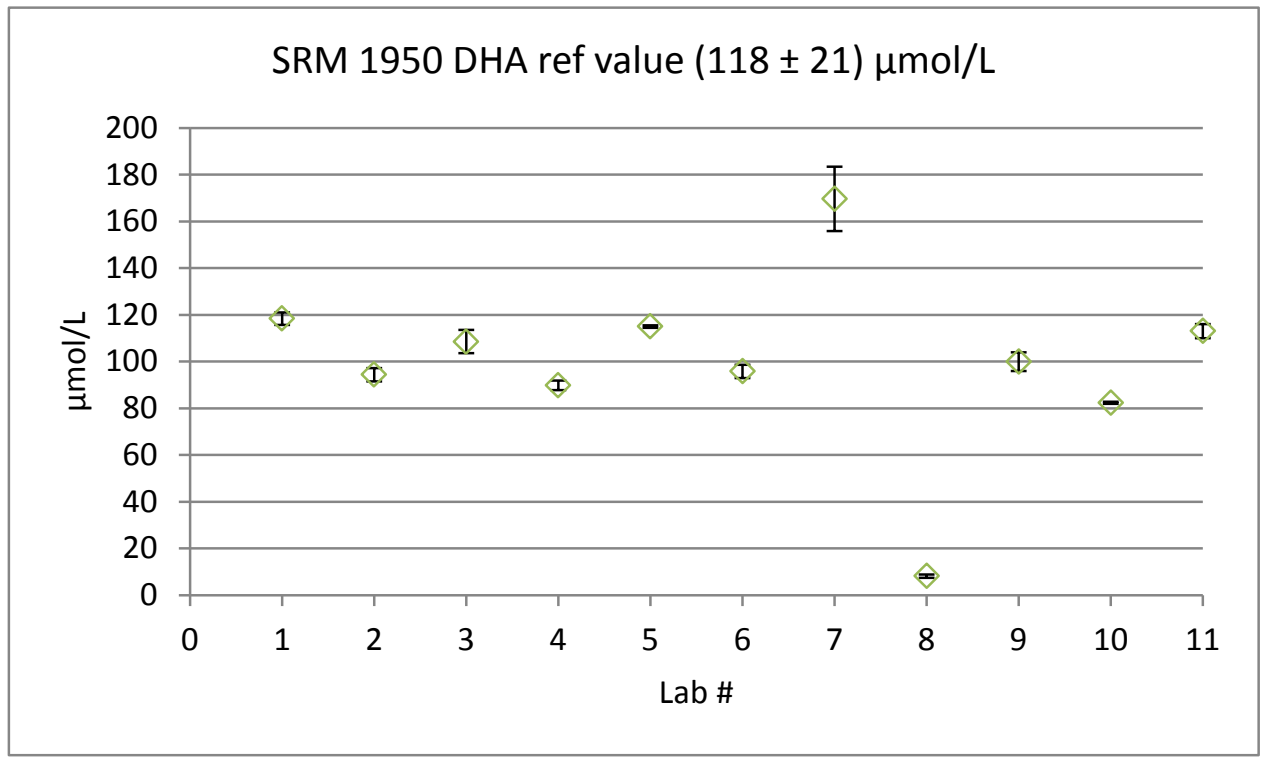

Median (excluding lab 8) $104 \mu \mathrm{mol} / \mathrm{L}$ 


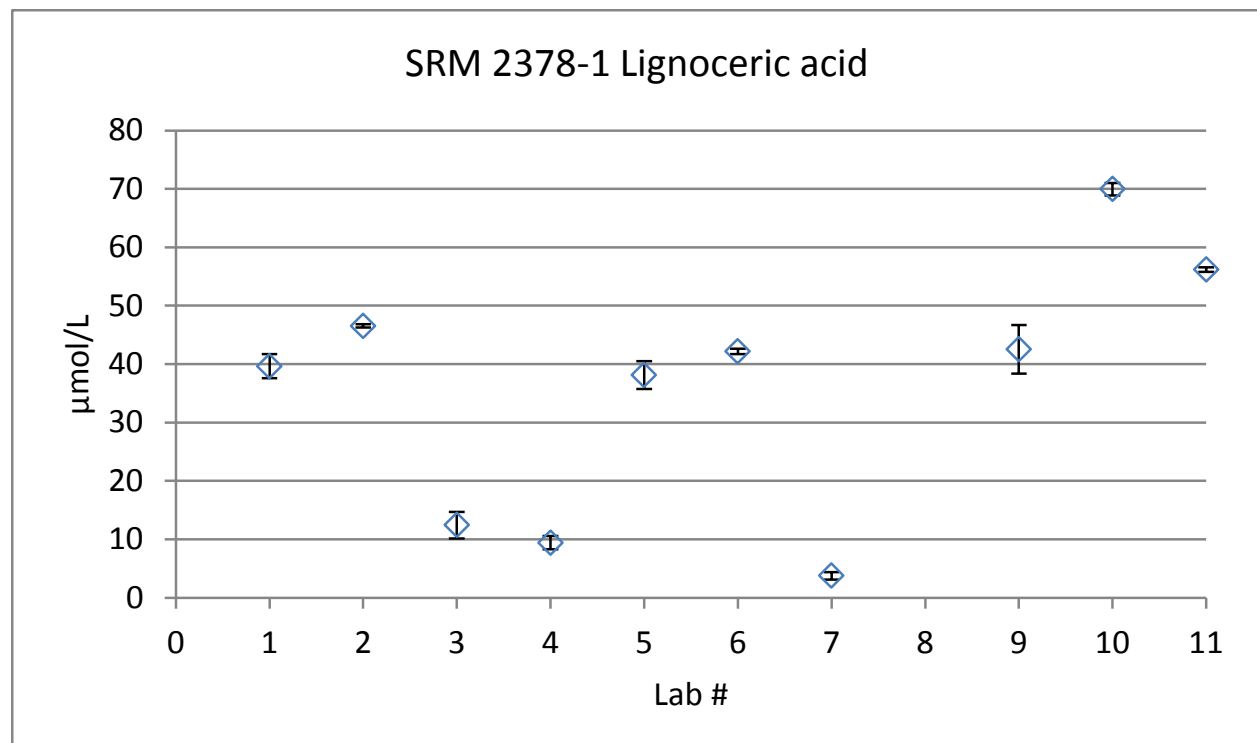

Median (excluding lab 8) $38.9 \mu \mathrm{mol} / \mathrm{L}$

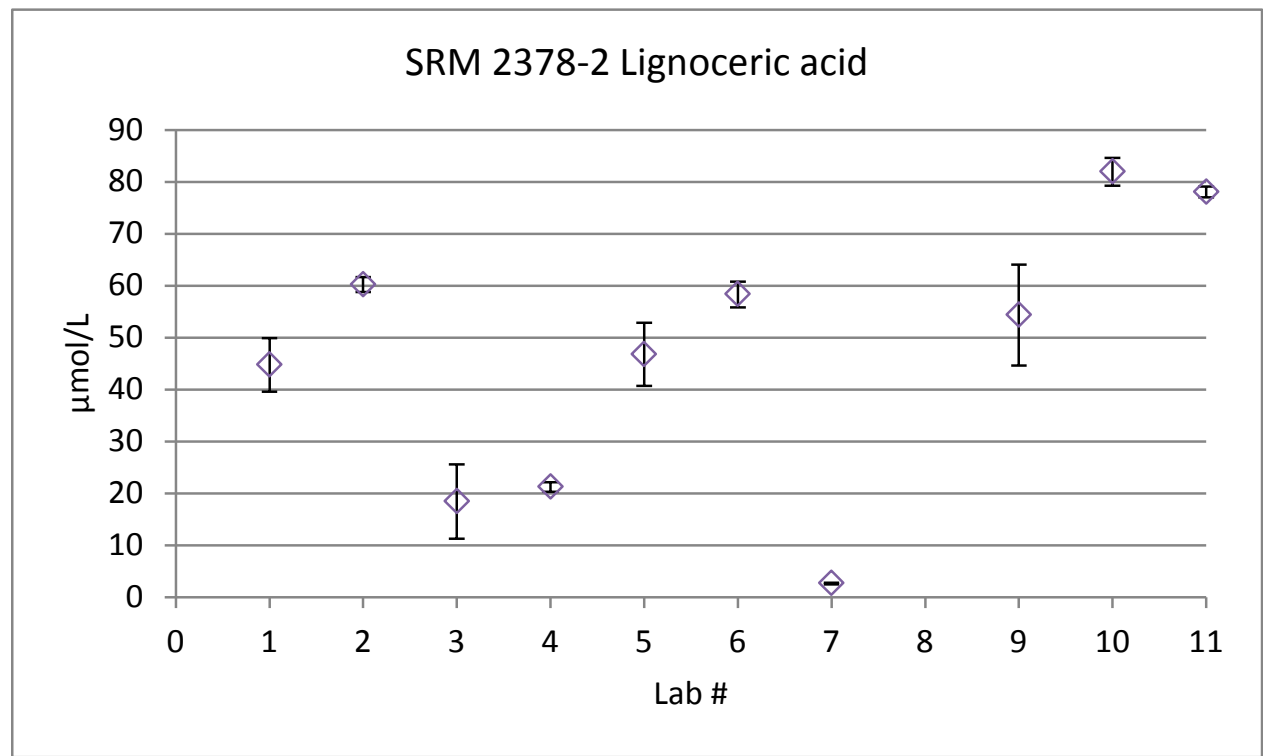

Median (excluding lab 8) $50.5 \mu \mathrm{mol} / \mathrm{L}$ 

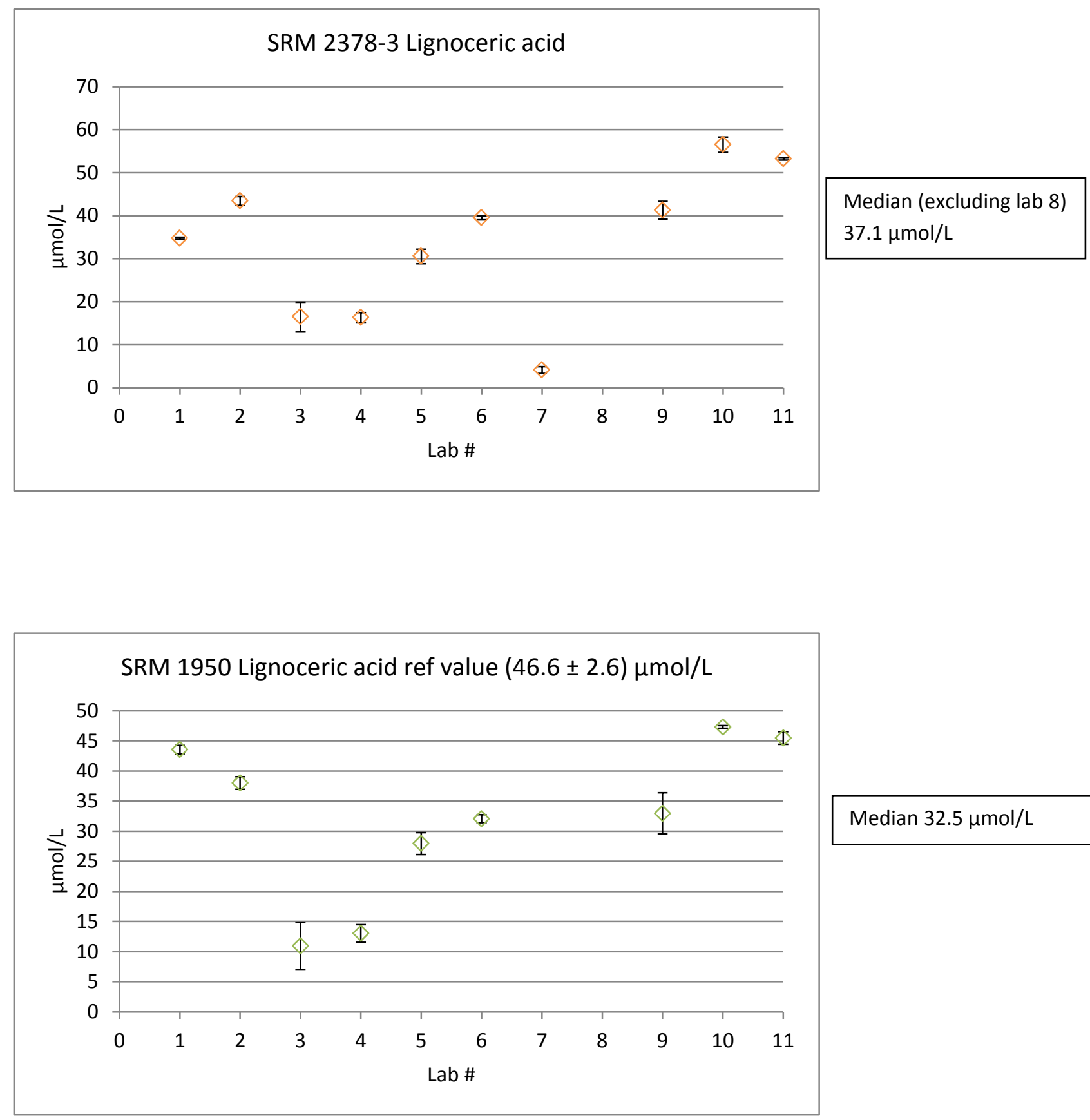

Median $32.5 \mu \mathrm{mol} / \mathrm{L}$ 


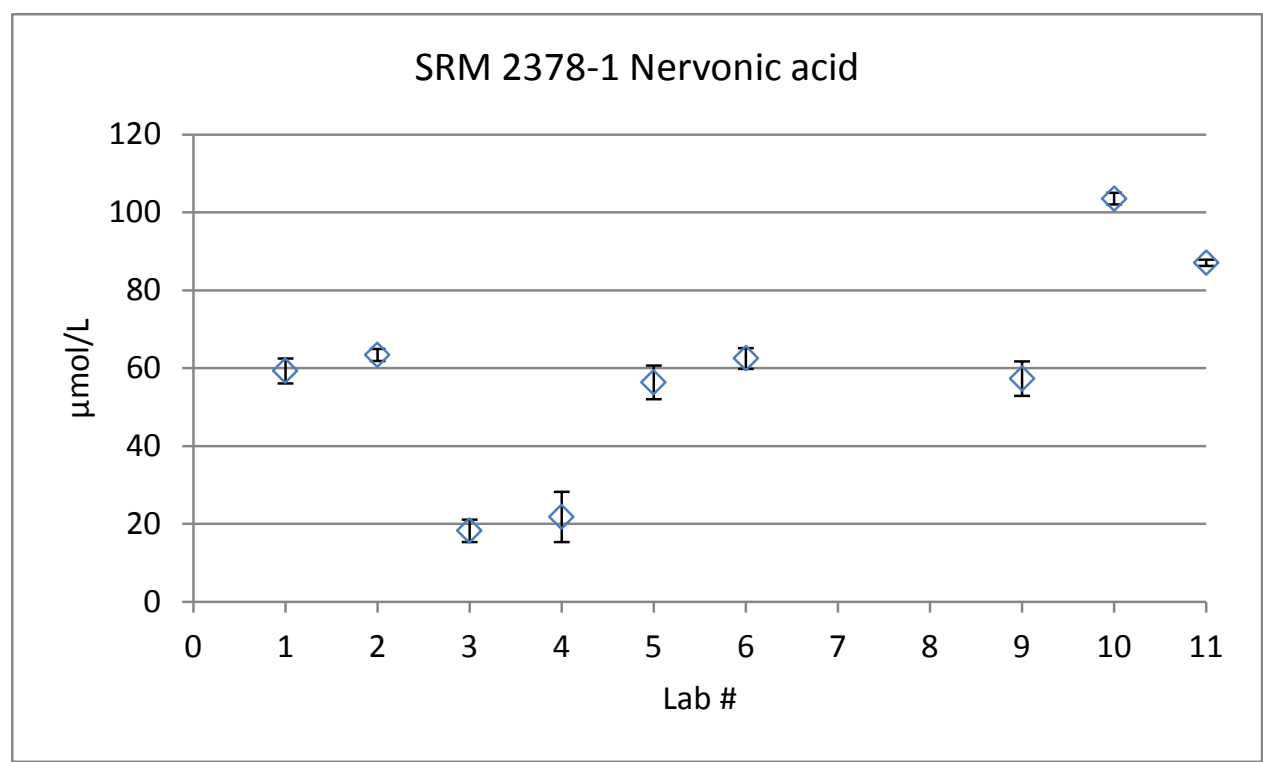

Median $57.3 \mu \mathrm{mol} / \mathrm{L}$

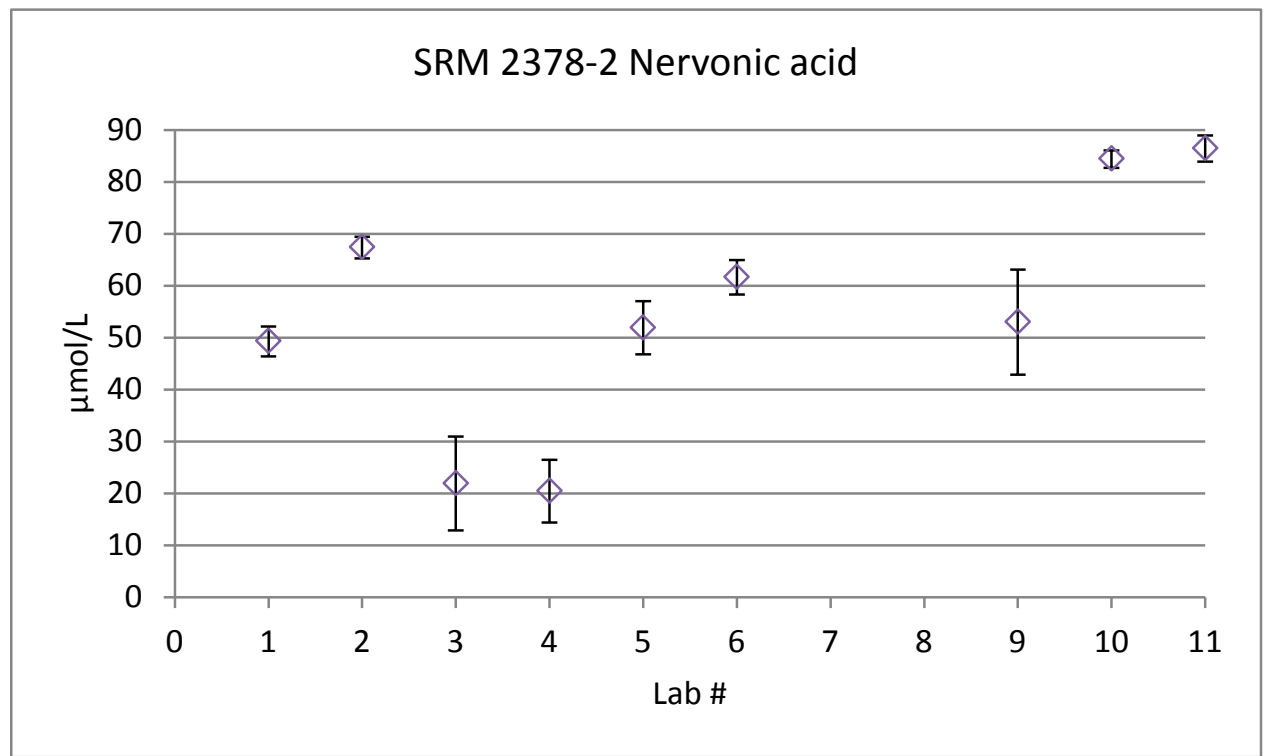

Median $52.9 \mu \mathrm{mol} / \mathrm{L}$ 


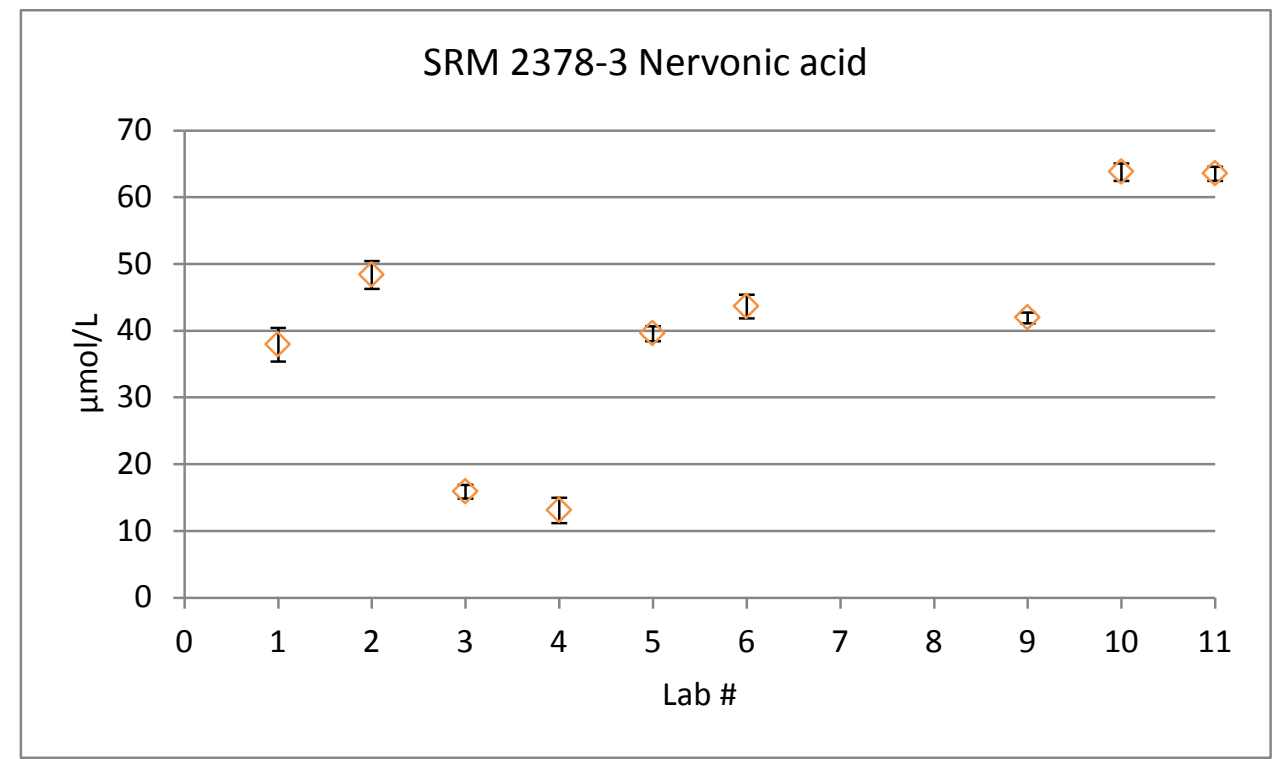

Median $41.9 \mu \mathrm{mol} / \mathrm{L}$

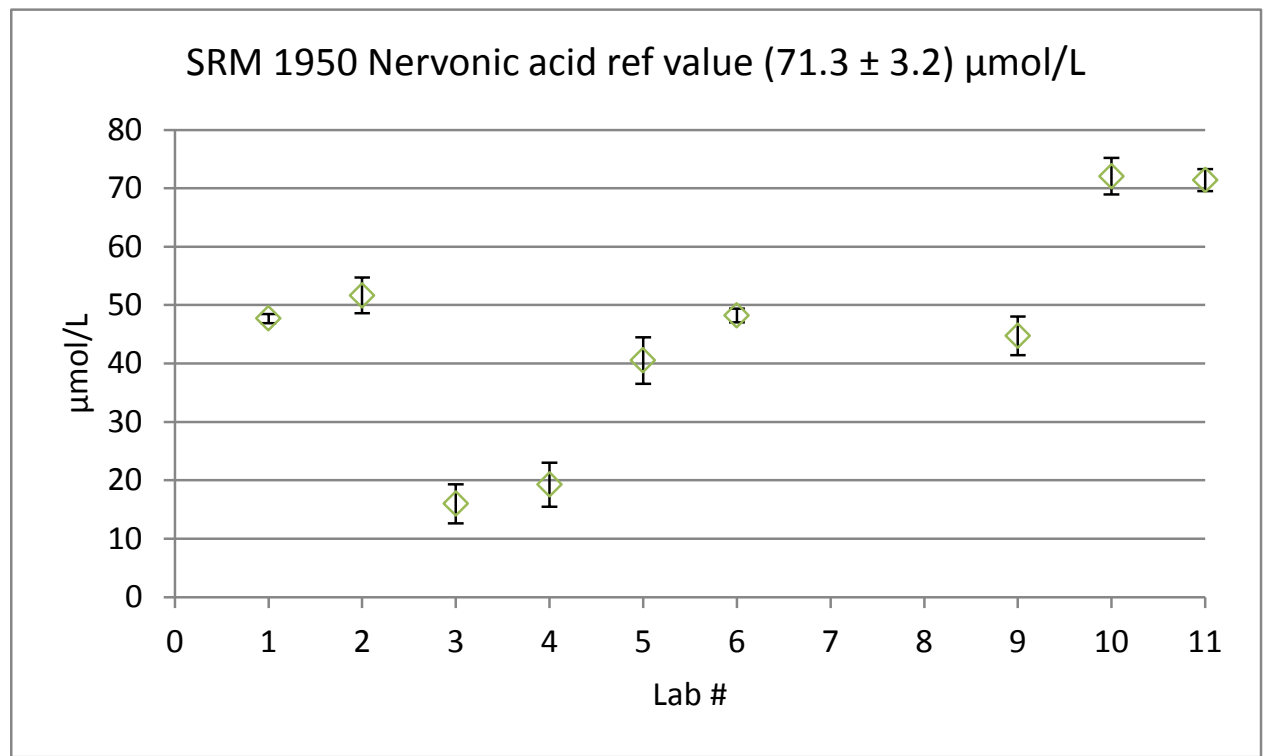

Median $47.7 \mu \mathrm{mol} / \mathrm{L}$ 
Table A-1 Procedures used

\begin{tabular}{|c|c|c|c|c|c|}
\hline & & & & & \\
\hline Reporting date & 10/19/2012 & $6 / 25 / 2012$ & 7/23/2012 & $7 / 29 / 2012$ & $7 / 31 / 2012$ \\
\hline Volume of sample extracted $(\mathrm{mL})$ : & & & $0.0125 \mathrm{~mL}$ & $0.0125 \mathrm{~mL}$ & \\
\hline \multicolumn{6}{|l|}{ Mass of samples extracted (g) (average of 3 ): } \\
\hline SRM 2378-1 & $0.93 \mathrm{~g}$ & $0.096 \mathrm{~g}$ & $0.012 \mathrm{~g}$ & & $0.100 \mathrm{~g}$ \\
\hline SRM 2378-2 & $0.90 \mathrm{~g}$ & $0.098 \mathrm{~g}$ & $0.013 \mathrm{~g}$ & & $0.100 \mathrm{~g}$ \\
\hline SRM 2378-3 & $0.90 \mathrm{~g}$ & $0.097 \mathrm{~g}$ & $0.014 \mathrm{~g}$ & & $0.100 \mathrm{~g}$ \\
\hline SRM 1950 & $0.90 \mathrm{~g}$ & $0.096 \mathrm{~g}$ & $0.013 \mathrm{~g}$ & & $0.100 \mathrm{~g}$ \\
\hline Analytical method reference, if available: & & \begin{tabular}{|} 
Lagerstadt SA, Hinrichs DR, Batt SM, Magera MJ, Rinaldo \\
P, McConnell IP. (2001) Quantitative determination of \\
plasma c8-C26 total fatty acids for the biochemical \\
diagnosis of nutritional and metabolic disorders. Mol \\
Genet Metab, 73:38--45
\end{tabular} & & & \\
\hline \multicolumn{6}{|l|}{ Analytical method used: } \\
\hline Hydrolysis method & $0.4 \mathrm{M}$ potassium hydroxide in methanol & Acid $(0.6 \mathrm{~N} \mathrm{HCl})$ and base $(1 \mathrm{~N} \mathrm{NaOH})$ hydrolysis & BF3 Methanol & BF3 Methanol & Folch \\
\hline Extraction method & liquid-liquid & Manual extraction & & & \\
\hline Extraction solvent & hexane & Hexane & & & 3mL of 2:1 chloroform:methanol \\
\hline Extraction time & $\begin{array}{l}\text { vortex for } 30 \mathrm{~min} \text { and centrifuge for } 5 \mathrm{~min} \text { - repeat } 3 \mathrm{x} \\
\text { with } 2 \mathrm{~mL} \text { of hexane each time }\end{array}$ & 2 minutes & & & 1 minute during vortex in 2:1 chloroform:methanol \\
\hline Extraction - other details & combine hexane phases & none & & & $\begin{array}{c}\text { Surrogate standard added to 2:1 chloroform:methanol } \\
\text { solution }\end{array}$ \\
\hline Sample extract cleanup method & & hexane extraction & & & $\begin{array}{l}\text { add 500ul sodium phosphate buffer, invert twice, } \\
\text { centrifuge @ 1734g for } 5 \text { min; extract bottom chloroform } \\
\text { layer, dry down under nitrogen, derivitize with } 1 \mathrm{~mL} 14 \% \\
\text { BF3 in methanol with 0.3mL hexane; Add } 1 \mathrm{~mL} \text { hexane, } \\
1 \mathrm{~mL} \text { water, vortex for } 1 \text { min, centrifuge @ } 1734 \mathrm{~g} \text { for } 3 \\
\text { min; Extract top hexane layer, dry down under nitrogen, } \\
\text { reconstitute in 65uL heptane }\end{array}$ \\
\hline Derivitization reagent & $\begin{array}{l}5 \% \text { ( } v / v) \text { sulfuric acid in methanol - heat } 80 \mathrm{deg} C \text { for } 30 \\
\text { min - extract } 3 \times \text { with } 2 \mathrm{~mL} \text { Lexane each time }\end{array}$ & Pentafluorobenzyl Bromide (PFBBr) & BF3 Methanol & BF3 Methanol & \\
\hline Analytical instrument & GC-FID & $\begin{array}{l}\text { Agilent } 6890 \text { series gas chromatograph with Agilent } 5973 \\
\text { Cl source MS }\end{array}$ & GC-FID & GC-FID & Varian 3900 \\
\hline Column phase & SP2560 & Crosslinked 5\% Diphenyl and 95\% Dimethylpolysiloxane & SP-2560 & SP-2560 & DB-FFAP \\
\hline Column length, $m$ & $100 \mathrm{~m}$ & 25 & 100 & 100 & 15 \\
\hline Column i.d., $\mathrm{mm}$ & 0.25 & 0.200 & 0.25 & 0.25 & 0.1 \\
\hline Column film thickness, $\mu \mathrm{m}$ & 0.25 & 0.33 & 0.2 & 0.2 & 0.1 \\
\hline Injection method (split, splitless, etc) & split 30:1 & split (split ratio 105:1) and splitless & Split & Split & split \\
\hline Method of quantitation: & & & & & \\
\hline$E S=$ external standards $(\mathrm{Y} / \mathrm{N})$ & & Y & Yes & $\mathrm{Y}$ & \\
\hline Number of ES used & & 10 & 3 & 3 & \\
\hline IS = internal standards $(Y / N)$ & y & $\mathrm{r}$ & Yes & r & y \\
\hline Number of IS used & 2 & 13 & 2 & 1 & 1 \\
\hline IS added PRIOR to extraction of sample (Y/N) & $\mathrm{y}$ & Y & Yes & $\mathrm{r}$ & $\mathrm{y}$ \\
\hline & & & & & \\
\hline Additional data/information & & & & & \\
\hline
\end{tabular}


Table A-1 Continued

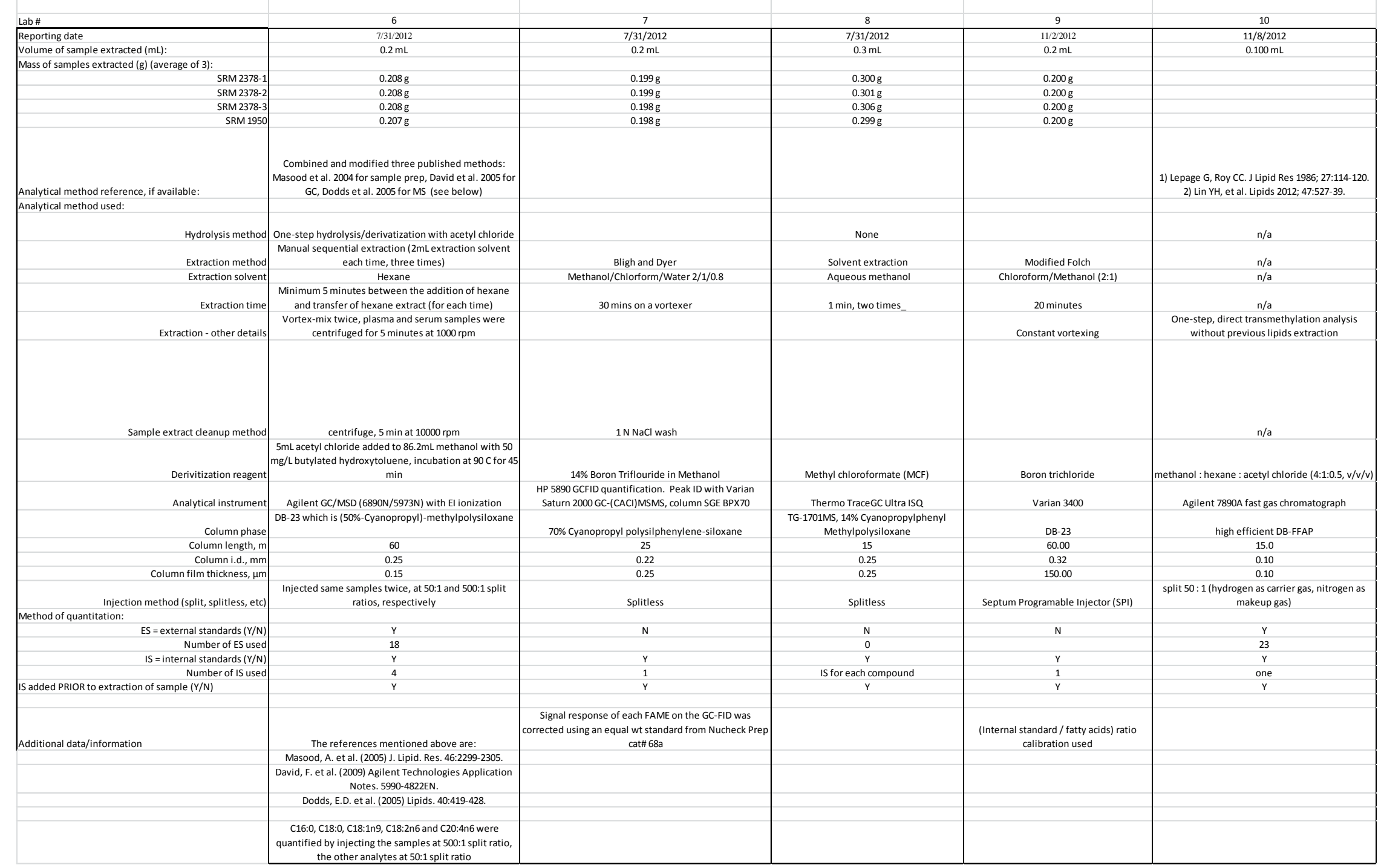




\begin{tabular}{|c|c|}
\hline Table A-1 Continued & \\
\hline Lab\# & 11 \\
\hline Reporting date & $1 / 10 / 2013$ \\
\hline Volume of sample extracted $(\mathrm{mL})$ : & $0.10 \mathrm{~mL}$ \\
\hline Mass of samples extracted (g) (average of 3): & \\
\hline SRM 2378-1 & $0.0967 \mathrm{~g}$ \\
\hline $\begin{array}{l}\text { SRM 2378-2 } \\
\text { SRM 2378-3. }\end{array}$ & $0.0983 \mathrm{~g}$ \\
\hline $\begin{array}{r}\text { SRM 2378-3 } \\
\text { SRM 1950 }\end{array}$ & $0.0987 \mathrm{~g}$ \\
\hline Analytical method reference, if available: & 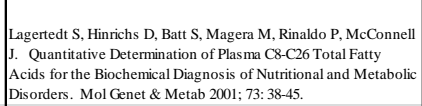 \\
\hline Hydrolysis method & Acid $(0.6 \mathrm{~N} \mathrm{HCl})$ and base $(1 \mathrm{~N} \mathrm{NaOH})$ hydrolysis \\
\hline $\begin{array}{r}\text { Extraction method } \\
\text { Extraction solvent }\end{array}$ & Liquid:liquid \\
\hline & hexane \\
\hline Extraction time & 20 to 30 minutes per extraction set \\
\hline Extraction - other details & triple extraction via automated liquid handler \\
\hline Sample extract cleanup method & Speedvac \\
\hline Derivitization reagent & PFBBr-pentafluorobenzyl bromide \\
\hline Analytical instrument & GC/MS \\
\hline Column phase & cyanopropyl-methylpolysiloxane phase (50-50) \\
\hline Column length, $m$ & 60 \\
\hline Column i.d., mm & 0.25 \\
\hline Column film thickness, $\mu \mathrm{m}$ & 0.25 \\
\hline Injection method (split, splititess, etc) & split \\
\hline Method of quantitation: & \\
\hline$E S=$ external standards $(Y / N)$ & $\mathrm{N}$ \\
\hline $\begin{array}{r}\text { Number of ES used } \\
\text { IS = internal standards }(\mathrm{Y} / \mathrm{N})\end{array}$ & Y \\
\hline $\begin{array}{l}\text { IS }=\text { internal standarass }(Y / N) \\
\text { Number of used }\end{array}$ & 18 \\
\hline Is added PRIOR to extraction of sample $(Y / N)$ & $\mathrm{r}$ \\
\hline Additional data/information & \\
\hline & \\
\hline & \\
\hline & \\
\hline & \\
\hline
\end{tabular}




\begin{tabular}{|c|c|c|c|c|c|c|c|c|c|}
\hline \multicolumn{10}{|c|}{ Table A-2 Calibration information } \\
\hline Lab & $\begin{array}{l}\text { Fatty Acid } \\
\text { Code }\end{array}$ & Fatty Acid Name & $\begin{array}{c}\text { Lowest } \\
\text { std } \\
(\mu \mathrm{mol} / \mathrm{L})\end{array}$ & $\begin{array}{c}\text { Highest } \\
\text { std } \\
(\mu \mathrm{mol} / \mathrm{L})\end{array}$ & $\begin{array}{c}\text { LOD } \\
(\mu \mathrm{mol} / \mathrm{L})\end{array}$ & $\begin{array}{c}\text { LOQ } \\
(\mu \mathrm{mol} / \mathrm{L})\end{array}$ & $\begin{array}{l}\text { Curve } \\
\text { Type }\end{array}$ & Weighting & IS used for quantitation \\
\hline \multirow[t]{24}{*}{ Lab 1} & C14:0 & Myristic acid & 5 & 500 & & & linear & & palmitic acid $-d 31$ \\
\hline & C14:1n5 & Myristoleic acid & 1.5 & 140 & & & linear & & palmitic acid $-d 31$ \\
\hline & C16:0 & Palmitic acid & 5.5 & 550 & & & linear & & palmitic acid $-d 31$ \\
\hline & C16:1n7 & Palmitoleic acid & 3.5 & 360 & & & linear & & palmitic acid $-d 31$ \\
\hline & C18:0 & Stearic acid & 5.5 & 560 & & & linear & & palmitic acid $-d 31$ \\
\hline & C18:1n7 & cis-Vaccenic acid & 1.5 & 175 & & & linear & & palmitic acid $-d 31$ \\
\hline & C18:1n9 & Oleic acid & 5.5 & 525 & & & linear & & palmitic acid $-d 31$ \\
\hline & C18:2n6 & Linoleic acid & 5.5 & 550 & & & linear & & palmitic acid $-d 31$ \\
\hline & C18:3n3 & alpha-Linolenic acid & 2.5 & 320 & & & linear & & palmitic acid $-d 31$ \\
\hline & C18:3n6 & gamma-Linolenic acid & 1 & 140 & & & linear & & palmitic acid $-d 31$ \\
\hline & C20:0 & Arachidic acid & 2.5 & 275 & & & linear & & palmitic acid $-d 31$ \\
\hline & C20:1n9 & 11-Eicosenoic acid & 1 & 150 & & & linear & & palmitic acid $-d 31$ \\
\hline & $\mathrm{C} 20: 2 \mathrm{n} 6$ & 11,14-Eicosadienoic acid & & & & & & & \\
\hline & $\mathrm{C} 20: 3 \mathrm{n} 6$ & homo-gamma-Linolenic acid & & & & & & & \\
\hline & C20:4n6 & Arachidonic acid & 1 & 150 & & & linear & & heneicosanoic acid \\
\hline & $\mathrm{C} 20: 5 \mathrm{n} 3$ & Eicosapentaenoic acid & 1 & 150 & & & linear & & heneicosanoic acid \\
\hline & C22:0 & Docosanoic acid & 3 & 320 & & & linear & & heneicosanoic acid \\
\hline & C22:1n9 & Docosenoic acid & 1.5 & 175 & & & linear & & heneicosanoic acid \\
\hline & $\mathrm{C} 22: 4 \mathrm{n} 6$ & Docosatetraenoic acid & & & & & & & \\
\hline & $\mathrm{C} 22: 5 \mathrm{n} 3$ & Docosapentaenoic acid & 1 & 150 & & & linear & & heneicosanoic acid \\
\hline & $\mathrm{C} 22: 5 \mathrm{n} 6$ & Docosapentaenoic acid & & & & & & & \\
\hline & $\mathrm{C} 22: 6 \mathrm{n} 3$ & Docosahexaenoic acid & 1 & 150 & & & linear & & heneicosanoic acid \\
\hline & C24:0 & Lignoceric acid & 1 & 150 & & & linear & & heneicosanoic acid \\
\hline & C24:1n9 & Nervonic acid & 1 & 150 & & & linear & & heneicosanoic acid \\
\hline \multirow[t]{13}{*}{ Lab 2} & C8:0 & Octanoic & 12.5 & 100 & 0.616 & ND & linear & equal & ${ }^{13} \mathrm{C}_{4}$-octanoic \\
\hline & C10:1 & Decenoic & 12.5 & 100 & 0.193 & ND & linear & equal & $\mathrm{d}_{3}$-decanoic \\
\hline & C10:0 & Decanoic & 12.5 & 100 & 0.193 & ND & linear & equal & $\mathrm{d}_{3}$-decanoic \\
\hline & C12:1 & Lauroleic & 12.5 & 100 & 0.642 & ND & linear & equal & $\mathrm{d}_{3}$-lauric \\
\hline & C12:0 & Lauric & 12.5 & 100 & 0.642 & ND & linear & equal & $\mathrm{d}_{3}$-lauric \\
\hline & C14:2 & Tetradecenoic & 12.5 & 100 & 0.048 & ND & linear & equal & $\mathrm{d}_{3}$-pentadecanoic \\
\hline & C14:1 & Myristoleic & 12.5 & 100 & 0.048 & ND & linear & equal & $\mathrm{d}_{3}$-pentadecanoic \\
\hline & C16:2 & Hexadecenoic & 12.5 & 100 & 0.048 & ND & linear & equal & $\mathrm{d}_{3}$-pentadecanoic \\
\hline & C20:0 & Arachidic & 125 & 1000 & 0.282 & ND & linear & equal & $\mathrm{d}_{3}$-arachidic \\
\hline & C20:3w9 & Mead & 6.25 & 50 & 0.023 & ND & linear & equal & $\mathrm{d}_{4}$-docosanoic \\
\hline & C20:3w6 & homo- $\gamma$-Linoleic & 6.25 & 50 & 0.023 & ND & linear & equal & $\mathrm{d}_{4}$-docosanoic \\
\hline & $\mathrm{C} 22: 5 \omega 3$ & Docosapentaenoic ( $\omega 3)$ & 6.25 & 50 & 0.021 & ND & linear & equal & $\mathrm{d}_{4}$-docosanoic \\
\hline & $\mathrm{C} 22: 5 \omega 6$ & Docosapentaenoic $(\omega 6)$ & 6.25 & 50 & 0.021 & ND & linear & equal & $\mathrm{d}_{4}$-docosanoic \\
\hline
\end{tabular}




\begin{tabular}{|c|c|c|c|c|c|c|c|c|c|}
\hline \multicolumn{10}{|c|}{ Table A-2 Continued } \\
\hline Lab & $\begin{array}{l}\text { Fatty Acid } \\
\text { Code }\end{array}$ & Fatty Acid Name & $\begin{array}{c}\text { Lowest } \\
\text { std } \\
\text { ( } \mu \mathrm{mol} / \mathrm{L})\end{array}$ & $\begin{array}{c}\text { Highest } \\
\text { std } \\
(\mu \mathrm{mol} / \mathrm{L})\end{array}$ & $\begin{array}{c}\text { LOD } \\
(\mu \mathrm{mol} / \mathrm{L})\end{array}$ & $\begin{array}{c}\text { LOQ } \\
(\mu \mathrm{mol} / \mathrm{L})\end{array}$ & $\begin{array}{l}\text { Curve } \\
\text { Type }\end{array}$ & Weighting & IS used for quantitation \\
\hline \multirow[t]{22}{*}{ Lab 2} & 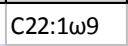 & Erucic & 6.25 & 50 & 0.022 & ND & linear & equal & $d_{4}$-docosanoic \\
\hline & C22:0 & Docosanoic & 6.25 & 50 & 0.055 & ND & linear & equal & $\mathrm{d}_{4}$-docosanoic \\
\hline & 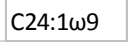 & Nervonic & 6.25 & 50 & 0.015 & ND & linear & equal & $\mathrm{d}_{4}$-tetracosanoic \\
\hline & C24:0 & Tetracosanoic & 6.25 & 50 & 0.054 & ND & linear & equal & $\mathrm{d}_{4}$-tetracosanoic \\
\hline & C26:1 & Hexacosenoic & 1.25 & 10 & 0.018 & ND & linear & equal & $\mathrm{d}_{4}$-hexacosanoic \\
\hline & C26:0 & Hexacosanoic & 1.25 & 10 & 0.018 & ND & linear & equal & $\mathrm{d}_{4}$-hexacosanoic \\
\hline & $\mathrm{PhA}$ & Phytanic & 2.5 & 20 & 0.008 & ND & linear & equal & $\mathrm{d}_{3}$-phytanic \\
\hline & PrA & Pristanic & 0.25 & 2.0 & 0.003 & ND & linear & equal & $\mathrm{d}_{3}$-pristanic \\
\hline & C14:0 & Myristic & 125 & 1000 & 0.783 & ND & linear & equal & $\mathrm{d}_{3}$-myristic \\
\hline & 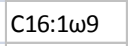 & 7-Hexadecenoic & 125 & 1000 & 0.057 & ND & linear & equal & $\mathrm{d}_{3}$-myristic \\
\hline & 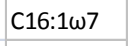 & Palmitoleic & 125 & 1000 & 0.057 & ND & linear & equal & $\mathrm{d}_{3}$-myristic \\
\hline & C16:0 & Palmitic & 250 & 2000 & 5.68 & ND & linear & equal & $\mathrm{d}_{3}$-palmitic \\
\hline & C18:2w6 & Linoleic & 250 & 2000 & 1.13 & ND & linear & equal & $\mathrm{d}_{3}$-stearic \\
\hline & 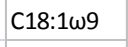 & Oleic & 250 & 2000 & 1.419 & ND & linear & equal & $\mathrm{d}_{3}$-stearic \\
\hline & $\mathrm{C} 18: 1 \omega 7$ & Vaccenic & 250 & 2000 & 1.419 & ND & linear & equal & $\mathrm{d}_{3}$-stearic \\
\hline & C18:0 & Stearic & 250 & 2000 & 8.312 & ND & linear & equal & $\mathrm{d}_{3}$-stearic \\
\hline & C18:3w6 & Y-Linolenic & 125 & 1000 & 0.042 & ND & linear & equal & $\mathrm{d}_{3}$-arachidic \\
\hline & C18:3w3 & Linolenic & 125 & 1000 & 0.042 & ND & linear & equal & $\mathrm{d}_{3}$-arachidic \\
\hline & $C 20: 5 \omega 3$ & Eicosapentaenoic & 125 & 1000 & 0.241 & ND & linear & equal & $\mathrm{d}_{3}$-arachidic \\
\hline & 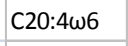 & Arachidonic & 125 & 1000 & 0.13 & ND & linear & equal & $\mathrm{d}_{3}$-arachidic \\
\hline & C22:6w3 & Docosahexaenoic & 6.25 & 50 & 0.029 & ND & linear & equal & $\mathrm{d}_{4}$-docosanoic \\
\hline & $\mathrm{C} 22: 4 \omega 6$ & Docosatetraenoic & 6.25 & 50 & 0.13 & ND & linear & equal & $\mathrm{d}_{4}$-docosanoic \\
\hline \multirow[t]{16}{*}{ Lab 3} & C14:0 & Myristic acid & 0.1 & 2.5 & & & Linear & None & \\
\hline & C16:0 & Palmitic acid & 1.6 & 40 & & & Linear & None & \\
\hline & $\mathrm{C} 16: 1 \mathrm{n} 7 \mathrm{t}$ & Palmitelaidic Acid & 0.05 & 1.25 & & & Linear & None & \\
\hline & C16:1n7 & Palmitoleic acid & 0.1 & 2.5 & & & Linear & None & \\
\hline & C17:0 & & 1 & 25 & & & Linear & None & \\
\hline & C18:0 & Stearic acid & 1.2 & 30 & & & Linear & None & \\
\hline & C18:1t & & 0.1 & 2.5 & & & Linear & None & \\
\hline & C18:1n9 & Oleic acid & 1.1 & 27.5 & & & Linear & None & \\
\hline & $\mathrm{C} 18: 2 \mathrm{n} 6 \mathrm{t}$ & Linoelaidic Acid & 0.05 & 1.25 & & & Linear & None & \\
\hline & C18:2n6 & Linoleic acid & 1 & 25 & & & Linear & None & \\
\hline & C20:0 & Arachidic acid & 0.1 & 2.5 & & & Linear & None & \\
\hline & C18:3n6 & gamma-Linolenic acid & 0.05 & 1.25 & & & Linear & None & \\
\hline & C20:1n9 & 11-Eicosenoic acid & 0.15 & 3.75 & & & Linear & None & \\
\hline & C18:3n3 & alpha-Linolenic acid & 0.05 & 1.25 & & & Linear & None & \\
\hline & $\mathrm{C} 20: 2 \mathrm{n} 6$ & 11,14-Eicosadienoic acid & 0.15 & 3.75 & & & Linear & None & \\
\hline & $\mathrm{C} 22: 0$ & Docosanoic acid & 0.1 & 2.5 & & & Linear & None & \\
\hline
\end{tabular}




\begin{tabular}{|c|c|c|c|c|c|c|c|c|c|}
\hline \multicolumn{10}{|c|}{ Table A-2 Continued } \\
\hline Lab & $\begin{array}{l}\text { Fatty Acid } \\
\text { Code }\end{array}$ & Fatty Acid Name & $\begin{array}{c}\text { Lowest } \\
\text { std } \\
\text { ( } \mu \mathrm{mol} / \mathrm{L})\end{array}$ & $\begin{array}{c}\text { Highest } \\
\text { std } \\
(\mu \mathrm{mol} / \mathrm{L})\end{array}$ & $\begin{array}{c}\text { LOD } \\
(\mu \mathrm{mol} / \mathrm{L})\end{array}$ & $\begin{array}{c}\mathrm{LOQ} \\
(\mu \mathrm{mol} / \mathrm{L})\end{array}$ & $\begin{array}{l}\text { Curve } \\
\text { Type }\end{array}$ & Weighting & IS used for quantitation \\
\hline \multirow[t]{13}{*}{ Lab 3} & $C 20: 3 n 6$ & homo-gamma-Linolenic acid & 0.3 & 7.5 & & & Linear & None & \\
\hline & $\mathrm{C} 20: 4 \mathrm{n} 6$ & Arachidonic acid & 1.2 & 30 & & & Linear & None & \\
\hline & C24:0 & Lignoceric acid & 0.1 & 2.5 & & & Linear & None & \\
\hline & $\mathrm{C} 20: 5 \mathrm{n} 3$ & Eicosapentaenoic acid & 0.3 & 7.5 & & & Linear & None & \\
\hline & C24:1n9 & Nervonic acid & 0.1 & 2.5 & & & Linear & None & \\
\hline & $\mathrm{C} 22: 3 \mathrm{n} 3$ & & 25 & 25 & & & Linear & None & \\
\hline & $\mathrm{C} 22: 4 \mathrm{n} 6$ & Docosatetraenoic acid & 0.2 & 5 & & & Linear & None & \\
\hline & $\mathrm{C} 22: 5 \mathrm{n} 6$ & Docosapentaenoic acid & 0.1 & 2.5 & & & Linear & None & \\
\hline & $\mathrm{C} 22: 5 \mathrm{n} 3$ & Docosapentaenoic acid & 0.3 & 7.5 & & & Linear & None & \\
\hline & $\mathrm{C} 22: 6 \mathrm{n} 3$ & Docosahexaenoic acid & 0.4 & 10 & & & Linear & None & \\
\hline & C14:1n5 & Myristoleic acid & & & & & & & \\
\hline & C18:1n7 & cis-Vaccenic acid & 1.1 & 27.5 & & & Linear & None & \\
\hline & C22:1n9 & Docosenoic acid & & & & & & & \\
\hline \multirow[t]{25}{*}{ Lab 4} & C14:0 & Myristic acid & 0.1 & 2.5 & & & Linear & None & \\
\hline & C16:0 & Palmitic acid & 1.6 & 40 & & & Linear & None & \\
\hline & $\mathrm{C} 16: 1 \mathrm{n} 7 \mathrm{t}$ & Palmitelaidic Acid & 0.05 & 1.25 & & & Linear & None & \\
\hline & C16:1n7 & Palmitoleic acid & 0.1 & 2.5 & & & Linear & None & \\
\hline & C18:0 & Stearic acid & 1.2 & 30 & & & Linear & None & \\
\hline & C18:1t & & 0.1 & 2.5 & & & Linear & None & \\
\hline & C18:1n9 & Oleic acid & 1.1 & 27.5 & & & Linear & None & \\
\hline & C18:2n6t & Linoelaidic Acid & 0.05 & 1.25 & & & Linear & None & \\
\hline & C18:2n6 & Linoleic acid & 1 & 25 & & & Linear & None & \\
\hline & C20:0 & Arachidic acid & 0.1 & 2.5 & & & Linear & None & \\
\hline & C18:3n6 & gamma-Linolenic acid & 0.05 & 1.25 & & & Linear & None & \\
\hline & C20:1n9 & 11-Eicosenoic acid & 0.15 & 3.75 & & & Linear & None & \\
\hline & C18:3n3 & alpha-Linolenic acid & 0.05 & 1.25 & & & Linear & None & \\
\hline & $\mathrm{C} 20: 2 \mathrm{n} 6$ & 11,14-Eicosadienoic acid & 0.15 & 3.75 & & & Linear & None & \\
\hline & $\mathrm{C} 22: 0$ & Docosanoic acid & 0.1 & 2.5 & & & Linear & None & \\
\hline & $C 20: 3 n 6$ & homo-gamma-Linolenic acid & 0.3 & 7.5 & & & Linear & None & \\
\hline & C20:4n6 & Arachidonic acid & 1.2 & 30 & & & Linear & None & \\
\hline & C24:0 & Lignoceric acid & 0.1 & 2.5 & & & Linear & None & \\
\hline & $C 20: 5 n 3$ & Eicosapentaenoic acid & 0.3 & 7.5 & & & Linear & None & \\
\hline & C24:1n9 & Nervonic acid & 0.1 & 2.5 & & & Linear & None & \\
\hline & $\mathrm{C} 22: 3 \mathrm{n} 3$ & & 25 & 25 & & & Linear & None & \\
\hline & $\mathrm{C} 22: 4 \mathrm{n} 6$ & Docosatetraenoic acid & 0.2 & 5 & & & Linear & None & \\
\hline & $\mathrm{C} 22: 5 \mathrm{n} 6$ & Docosapentaenoic acid & 0.1 & 2.5 & & & Linear & None & \\
\hline & $C 22: 5 n 3$ & Docosapentaenoic acid & 0.3 & 7.5 & & & Linear & None & \\
\hline & $\mathrm{C} 22: 6 \mathrm{n} 3$ & Docosahexaenoic acid & 0.4 & 10 & & & Linear & None & \\
\hline
\end{tabular}




\begin{tabular}{|c|c|c|c|c|c|c|c|c|c|}
\hline \multicolumn{10}{|c|}{ Table A-2 Continued } \\
\hline Lab & $\begin{array}{l}\text { Fatty Acid } \\
\text { Code }\end{array}$ & Fatty Acid Name & $\begin{array}{c}\text { Lowest } \\
\text { std } \\
(\mu \mathrm{mol} / \mathrm{L})\end{array}$ & $\begin{array}{c}\text { Highest } \\
\text { std } \\
(\mu \mathrm{mol} / \mathrm{L})\end{array}$ & $\begin{array}{c}\text { LOD } \\
(\mu \mathrm{mol} / \mathrm{L})\end{array}$ & $\begin{array}{c}\text { LOQ } \\
(\mu \mathrm{mol} / \mathrm{L})\end{array}$ & $\begin{array}{l}\text { Curve } \\
\text { Type }\end{array}$ & Weighting & IS used for quantitation \\
\hline Lab 5 & \multicolumn{2}{|c|}{ No information } & & & & & & & \\
\hline \multirow[t]{18}{*}{ Lab 6} & C14:0 & Myristic & 12.60 & 440.63 & 1.09 & & quadratic & $1 / x$ & C13:1n1 FAME \\
\hline & C16:0 & Palmitic & 233.66 & 7379.21 & 35.81 & & quadratic & $1 / x$ & C17:1n7 trans FAME \\
\hline & C16:1n7 & Palmitoleic & 23.47 & 740.53 & 4.03 & & quadratic & $1 / x$ & C13:1n1 FAME \\
\hline & C18:0 & Stearic & 100.11 & 3503.59 & 6.97 & & quadratic & $1 / x$ & C17:1n7 trans FAME \\
\hline & C18:1n9 & Oleic & 214.02 & 6758.48 & 36.77 & & quadratic & $1 / x$ & C17:1n7 trans FAME \\
\hline & C18:1n7 & Vaccenic & 21.31 & 673.38 & 2.49 & & quadratic & $1 / x$ & C21:0 FAME \\
\hline & C18:2n6 & Linoleic & 214.76 & 6782.35 & 26.76 & & quadratic & $1 / x$ & C17:1n7 trans FAME \\
\hline & C18:3n6 & gamma-Linolenic & 10.34 & 361.85 & 0.83 & & quadratic & $1 / x$ & C21:0 FAME \\
\hline & C18:3n3 & alpha-Linolenic & 10.24 & 357.83 & 1.22 & & quadratic & $1 / x$ & C21:0 FAME \\
\hline & C20:3n6 & Eicosatrienoic & 20.03 & 632.10 & 2.90 & & quadratic & $1 / x$ & C21:0 FAME \\
\hline & $\mathrm{C} 20: 4 \mathrm{n} 6$ & Arachidonic & 92.68 & 3243.48 & 5.07 & & quadratic & $1 / x$ & C17:1n7 trans FAME \\
\hline & C22:0 & Behenic & 8.25 & 288.84 & 0.66 & & quadratic & $1 / x$ & C21:0 FAME \\
\hline & $C 20: 5 n 3$ & Eicosapentaenoic & 9.59 & 335.07 & 1.41 & & quadratic & $1 / x$ & C23:1n9 FAME \\
\hline & C22:4n6 & Docosatetraenoic & 8.60 & 300.90 & 0.70 & & quadratic & $1 / x$ & C23:1n9 FAME \\
\hline & C24:0 & Lignoceric & 7.81 & 272.99 & 0.55 & & quadratic & $1 / x$ & C23:1n9 FAME \\
\hline & $C 22: 5 n 3$ & Docosapentaenoic & 8.62 & 301.39 & 0.67 & & quadratic & $1 / x$ & C23:1n9 FAME \\
\hline & C24:1 & Nervonic & 7.72 & 270.62 & 1.14 & & quadratic & $1 / x$ & C23:1n9 FAME \\
\hline & $\mathrm{C} 22: 6 \mathrm{n} 3$ & Docosahexaenoic & 18.48 & 583.11 & 1.76 & & quadratic & $1 / x$ & C23:1n9 FAME \\
\hline Lab 7 & \multicolumn{2}{|c|}{ No information } & & & & & & & \\
\hline \multirow[t]{18}{*}{ Lab 8} & C14:0 & Myristic & 0.66 & 876.39 & 1.12 & 3.39 & linear & & myristic-d3 \\
\hline & C14:1n5 & Myristoleic & 0.33 & 442.11 & 0.92 & 2.79 & linear & & myristoleic-d3 \\
\hline & C16:0 & Palmitic & 0.88 & 870.78 & 2.63 & 7.97 & linear & & palmitic-d3 \\
\hline & C16:1n7 & Palmitoleic & 0.59 & 393.36 & 0.84 & 2.55 & linear & & palmitoleic-d3 \\
\hline & C18:0 & Stearic & 1.06 & 703.56 & 2.74 & 8.30 & linear & & stearic-d3 \\
\hline & C18:1n7 & cis-Vaccenic & 1.06 & 598.32 & 1.36 & 4.12 & linear & & cis-vaccenic-d3 \\
\hline & C18:1n9 & Oleic & 1.07 & 708.57 & 1.79 & 5.42 & linear & & oleic-d3 \\
\hline & C18:2n6 & Linoleic & 1.35 & 356.84 & 0.97 & 2.94 & linear & & linoleic-d3 \\
\hline & C18:3n3 & alpha-Linolenic & 1.16 & 412.79 & 1.02 & 3.09 & linear & & alpha-linolenic-d3 \\
\hline & C18:3n6 & gamma-Linolenic & 1.36 & 459.43 & 1.01 & 3.06 & linear & & gamma-linolenic-d3 \\
\hline & C20:0 & Arachidic & 0.97 & 640.41 & 2.54 & 7.70 & linear & & arachidic-d3 \\
\hline & C20:1n9 & 11-Eicosenoic & 0.81 & 422.28 & 2.03 & 6.15 & linear & & 11-eicosenoic-d3 \\
\hline & $\mathrm{C} 20: 2 \mathrm{n} 6$ & 11,14-Eicosadienoic & 0.82 & 424.39 & 1.03 & 3.12 & linear & & 11,14-eicosadienoic-d3 \\
\hline & $\mathrm{C} 20: 3 \mathrm{n} 6$ & h-g-Linolenic & 0.51 & 415.94 & 0.96 & 2.91 & linear & & h-g-linolenic-d3 \\
\hline & $\mathrm{C} 20: 4 \mathrm{n} 6$ & Arachidonic & 0.83 & 428.69 & 1.06 & 3.21 & linear & & arachidonic-d3 \\
\hline & $C 20: 5 n 3$ & Eicosapentaenoic & 0.89 & 487.37 & 1.23 & 3.73 & linear & & eicosapentaenoic-d3 \\
\hline & C22:0 & Docosanoic & 1.19 & 521.24 & 1.08 & 3.27 & linear & & docosanoic-d3 \\
\hline & C22:1n9 & Docosenoic & 1.25 & 654.40 & 1.23 & 3.73 & linear & & docosenoic-d3 \\
\hline
\end{tabular}




\begin{tabular}{|c|c|c|c|c|c|c|c|c|c|}
\hline \multicolumn{10}{|c|}{ Table A-2 Continued } \\
\hline Lab & $\begin{array}{l}\text { Fatty Acid } \\
\text { Code }\end{array}$ & Fatty Acid Name & $\begin{array}{c}\text { Lowest } \\
\text { std } \\
(\mu \mathrm{mol} / \mathrm{L})\end{array}$ & $\begin{array}{c}\text { Highest } \\
\text { std } \\
(\mu \mathrm{mol} / \mathrm{L})\end{array}$ & $\begin{array}{c}\text { LOD } \\
(\mu \mathrm{mol} / \mathrm{L})\end{array}$ & $\begin{array}{c}\text { LOQ } \\
(\mu \mathrm{mol} / \mathrm{L})\end{array}$ & $\begin{array}{l}\text { Curve } \\
\text { Type }\end{array}$ & Weighting & IS used for quantitation \\
\hline \multirow[t]{5}{*}{ Lab 8} & $\mathrm{C} 22: 4 \mathrm{n} 6$ & Docosatetraenoic & 1.24 & 459.37 & 0.5 & 1.52 & linear & & docosatetraenoic-d3 \\
\hline & $C 22: 5 n 3$ & Docosapentaenoic & 1.26 & 423.37 & 0.48 & 1.45 & linear & & docosapentaenoic-d3 \\
\hline & $\mathrm{C} 22: 6 \mathrm{n} 3$ & Docosahexaenoic & 0.77 & 504.66 & 0.89 & 2.70 & linear & & docosahexaenoic-d3 \\
\hline & C24:0 & Lignoceric & 1.02 & 542.93 & 2.78 & 8.42 & linear & & lignoceric - d3 \\
\hline & C24:1n9 & Nervonic & 1.03 & 472.96 & 1.25 & 3.79 & linear & & nervonic-d3 \\
\hline \multirow[t]{28}{*}{ Lab 9} & C14:0 & & & & & & & 1.0097 & tritridecanoin \\
\hline & C14:1 & & & & & & & 1.0012 & tritridecanoin \\
\hline & C15:0 & & & & & & & 1.0207 & tritridecanoin \\
\hline & C16:0 & & & & & & & 1.0214 & tritridecanoin \\
\hline & C16:1 & & & & & & & 1.0337 & tritridecanoin \\
\hline & C18:0 & & & & & & & 1.0370 & tritridecanoin \\
\hline & C18:1 total & & & & & & & 1.0382 & tritridecanoin \\
\hline & C18:2n6 & & & & & & & 1.0432 & tritridecanoin \\
\hline & C18:3n6 & & & & & & & 1.0492 & tritridecanoin \\
\hline & C18:3n3 & & & & & & & 1.0511 & tritridecanoin \\
\hline & C18:4n3 & & & & & & & & tritridecanoin \\
\hline & C20:0 & & & & & & & 1.0129 & tritridecanoin \\
\hline & C20:1 total & & & & & & & 1.0159 & tritridecanoin \\
\hline & $\mathrm{C} 20: 2 \mathrm{n} 6$ & & & & & & & 1.0266 & tritridecanoin \\
\hline & $\mathrm{C} 20: 3 \mathrm{n} 6$ & & & & & & & 1.0222 & tritridecanoin \\
\hline & C20:4n6 & & & & & & & 1.0521 & tritridecanoin \\
\hline & $C 20: 3 n 3$ & & & & & & & 1.0121 & tritridecanoin \\
\hline & $\mathrm{C} 20: 4 \mathrm{n} 3$ & & & & & & & & tritridecanoin \\
\hline & $C 20: 5 n 3$ & & & & & & & 1.0341 & tritridecanoin \\
\hline & $\mathrm{C} 22: 0$ & & & & & & & 0.9930 & tritridecanoin \\
\hline & C22:1total & & & & & & & 0.9914 & tritridecanoin \\
\hline & $\mathrm{C} 22: 2 \mathrm{n} 6$ & & & & & & & 1.0039 & tritridecanoin \\
\hline & $\mathrm{C} 22: 4 \mathrm{n} 6$ & & & & & & & 1.0051 & tritridecanoin \\
\hline & $C 22: 5 n 6$ & & & & & & & 1.0324 & tritridecanoin \\
\hline & $\mathrm{C} 22: 5 \mathrm{n} 3$ & & & & & & & 0.9949 & tritridecanoin \\
\hline & $\mathrm{C} 22: 6 \mathrm{n} 3$ & & & & & & & 0.9825 & tritridecanoin \\
\hline & C24:0 & & & & & & & 0.9862 & tritridecanoin \\
\hline & C24:1 & & & & & & & 0.9740 & tritridecanoin \\
\hline \multirow[t]{5}{*}{ Lab 10} & C14:0 & Myristic acid & $\mathrm{n} / \mathrm{a}$ & 277 & $\mathrm{n} / \mathrm{a}$ & 2.76 & one point & $\mathrm{n} / \mathrm{a}$ & 22:3n-3 ethyl ester \\
\hline & C14:1n5 & Myristoleic acid & $\mathrm{n} / \mathrm{a}$ & 277 & $\mathrm{n} / \mathrm{a}$ & $\mathrm{n} / \mathrm{a}$ & one point & $\mathrm{n} / \mathrm{a}$ & 22:3n-3 ethyl ester \\
\hline & C16:0 & Palmitic acid & $\mathrm{n} / \mathrm{a}$ & 277 & $\mathrm{n} / \mathrm{a}$ & 2.76 & one point & $\mathrm{n} / \mathrm{a}$ & $22: 3 n-3$ ethyl ester \\
\hline & C16:1n7 & Palmitoleic acid & $\mathrm{n} / \mathrm{a}$ & 277 & $\mathrm{n} / \mathrm{a}$ & 2.76 & one point & $\mathrm{n} / \mathrm{a}$ & 22:3n-3 ethyl ester \\
\hline & C18:0 & Stearic acid & $\mathrm{n} / \mathrm{a}$ & 277 & $\mathrm{n} / \mathrm{a}$ & 2.76 & one point & $\mathrm{n} / \mathrm{a}$ & 22:3n-3 ethyl ester \\
\hline
\end{tabular}




\begin{tabular}{|c|c|c|c|c|c|c|c|c|c|}
\hline \multicolumn{10}{|c|}{ Table A-2 Continued } \\
\hline Lab & $\begin{array}{l}\text { Fatty Acid } \\
\text { Code }\end{array}$ & Fatty Acid Name & $\begin{array}{c}\text { Lowest } \\
\text { std } \\
(\mu \mathrm{mol} / \mathrm{L})\end{array}$ & $\begin{array}{c}\text { Highest } \\
\text { std } \\
(\mu \mathrm{mol} / \mathrm{L})\end{array}$ & $\begin{array}{c}\text { LOD } \\
(\mu \mathrm{mol} / \mathrm{L})\end{array}$ & $\begin{array}{c}\text { LOQ } \\
(\mu \mathrm{mol} / \mathrm{L})\end{array}$ & $\begin{array}{l}\text { Curve } \\
\text { Type }\end{array}$ & Weighting & IS used for quantitation \\
\hline \multirow[t]{19}{*}{ Lab 10} & C18:1n7 & cis-Vaccenic acid & $\mathrm{n} / \mathrm{a}$ & 277 & $n / a$ & 2.76 & one point & $\mathrm{n} / \mathrm{a}$ & 22:3n-3 ethyl ester \\
\hline & C18:1n9 & Oleic acid & $\mathrm{n} / \mathrm{a}$ & 277 & $\mathrm{n} / \mathrm{a}$ & 2.76 & one point & $\mathrm{n} / \mathrm{a}$ & 22:3n-3 ethyl ester \\
\hline & $\mathrm{C} 18: 2 \mathrm{n} 6$ & Linoleic acid & $\mathrm{n} / \mathrm{a}$ & 277 & $\mathrm{n} / \mathrm{a}$ & 2.76 & one point & $\mathrm{n} / \mathrm{a}$ & 22:3n-3 ethyl ester \\
\hline & $\mathrm{C} 18: 3 \mathrm{n} 3$ & alpha-Linolenic acid & $\mathrm{n} / \mathrm{a}$ & 277 & $\mathrm{n} / \mathrm{a}$ & 2.76 & one point & $\mathrm{n} / \mathrm{a}$ & 22:3n-3 ethyl ester \\
\hline & $\mathrm{C} 18: 3 \mathrm{n} 6$ & gamma-Linolenic acid & $\mathrm{n} / \mathrm{a}$ & 277 & $\mathrm{n} / \mathrm{a}$ & 2.76 & one point & $\mathrm{n} / \mathrm{a}$ & 22:3n-3 ethyl ester \\
\hline & $\mathrm{C} 20: 0$ & Arachidic acid & $\mathrm{n} / \mathrm{a}$ & 277 & $\mathrm{n} / \mathrm{a}$ & 2.76 & one point & $\mathrm{n} / \mathrm{a}$ & 22:3n-3 ethyl ester \\
\hline & $\mathrm{C} 20: \ln 9$ & 11-Eicosenoic acid & $\mathrm{n} / \mathrm{a}$ & 277 & $\mathrm{n} / \mathrm{a}$ & 2.76 & one point & $\mathrm{n} / \mathrm{a}$ & 22:3n-3 ethyl ester \\
\hline & $C 20: 2 n 6$ & 11,14-Eicosadienoic acid & $\mathrm{n} / \mathrm{a}$ & 277 & $\mathrm{n} / \mathrm{a}$ & 2.76 & one point & $\mathrm{n} / \mathrm{a}$ & 22:3n-3 ethyl ester \\
\hline & $C 20: 3 n 6$ & homo-gamma-Linolenic acid & $\mathrm{n} / \mathrm{a}$ & 277 & $\mathrm{n} / \mathrm{a}$ & 2.76 & one point & $\mathrm{n} / \mathrm{a}$ & 22:3n-3 ethyl ester \\
\hline & $\mathrm{C} 20: 4 \mathrm{n} 6$ & Arachidonic acid & $\mathrm{n} / \mathrm{a}$ & 277 & $\mathrm{n} / \mathrm{a}$ & 2.76 & one point & $\mathrm{n} / \mathrm{a}$ & $22: 3 n-3$ ethyl ester \\
\hline & $C 20: 5 n 3$ & Eicosapentaenoic acid & $\mathrm{n} / \mathrm{a}$ & 277 & $\mathrm{n} / \mathrm{a}$ & 2.76 & one point & $\mathrm{n} / \mathrm{a}$ & $22: 3 n-3$ ethyl ester \\
\hline & C22:0 & Docosanoic acid & $\mathrm{n} / \mathrm{a}$ & 277 & $\mathrm{n} / \mathrm{a}$ & 2.76 & one point & $\mathrm{n} / \mathrm{a}$ & 22:3n-3 ethyl ester \\
\hline & $\mathrm{C} 22: \ln 9$ & Docosenoic acid & $\mathrm{n} / \mathrm{a}$ & 277 & $n / a$ & 2.76 & one point & $\mathrm{n} / \mathrm{a}$ & $22: 3 n-3$ ethyl ester \\
\hline & C22:4n6 & Docosatetraenoic acid & $\mathrm{n} / \mathrm{a}$ & 277 & $n / a$ & 2.76 & one point & $n / a$ & 22:3n-3 ethyl ester \\
\hline & $C 22: 5 n 3$ & Docosapentaenoic acid & $\mathrm{n} / \mathrm{a}$ & 277 & $\mathrm{n} / \mathrm{a}$ & 2.76 & one point & $\mathrm{n} / \mathrm{a}$ & 22:3n-3 ethyl ester \\
\hline & $C 22: 5 n 6$ & Docosapentaenoic acid & $\mathrm{n} / \mathrm{a}$ & 277 & $\mathrm{n} / \mathrm{a}$ & $\mathrm{n} / \mathrm{a}$ & $\mathrm{n} / \mathrm{a}$ & $\mathrm{n} / \mathrm{a}$ & 22:3n-3 ethyl ester \\
\hline & C22:6n3 & Docosahexaenoic acid & $n / a$ & 277 & $n / a$ & 2.76 & one point & $n / a$ & 22:3n-3 ethyl ester \\
\hline & C24:0 & Lignoceric acid & $\mathrm{n} / \mathrm{a}$ & 277 & $\mathrm{n} / \mathrm{a}$ & 2.76 & one point & $n / a$ & 22:3n-3 ethyl ester \\
\hline & C24:1n9 & Nervonic acid & $\mathrm{n} / \mathrm{a}$ & 277 & $\mathrm{n} / \mathrm{a}$ & 2.76 & one point & $\mathrm{n} / \mathrm{a}$ & 22:3n-3 ethyl ester \\
\hline \multirow[t]{34}{*}{ Lab 11} & $\mathrm{C} 8: 0$ & Caprylic (octanoic) & 1.06 & 91.3 & $n / a$ & $n / a$ & linear & $1 / x$ & Caprylic-d3 \\
\hline & C10:0 & Capric (decanoic) & 0.51 & 45.7 & $\mathrm{n} / \mathrm{a}$ & $\mathrm{n} / \mathrm{a}$ & quadratic & $1 / x^{2}$ & Capric-d3 \\
\hline & C12:0 & Lauric (dodecanoic) & 1.00 & 84.0 & $\mathrm{n} / \mathrm{a}$ & $\mathrm{n} / \mathrm{a}$ & quadratic & $1 / x^{2}$ & Lauric-d3 \\
\hline & C14:0 & Myristic & 10.8 & 1075 & $\mathrm{n} / \mathrm{a}$ & $\mathrm{n} / \mathrm{a}$ & quadratic & $1 / x^{2}$ & Myristic-d27 \\
\hline & C14:1n5 & Myristoleic & 1.49 & 149 & $\mathrm{n} / \mathrm{a}$ & $\mathrm{n} / \mathrm{a}$ & quadratic & $1 / x^{2}$ & Myristic-d27 \\
\hline & C15:0 & Pentadecanoic & 0.50 & 54.7 & $\mathrm{n} / \mathrm{a}$ & $\mathrm{n} / \mathrm{a}$ & linear & $1 / x^{2}$ & Pentadecanoic-d3 \\
\hline & C16:1n7 & Palmitoleic & 25.3 & 2530 & $n / a$ & $\mathrm{n} / \mathrm{a}$ & quadratic & $1 / x^{2}$ & Palmitoleic-d14 \\
\hline & C16:0 & Palmitic & 95.9 & 9591 & $\mathrm{n} / \mathrm{a}$ & $\mathrm{n} / \mathrm{a}$ & quadratic & $1 / x^{2}$ & Palmitic-d31 \\
\hline & C17:0 & Margaric (heptadecanoic) & 2.85 & 52.2 & $\mathrm{n} / \mathrm{a}$ & $\mathrm{n} / \mathrm{a}$ & quadratic & $1 / x$ & Margaric-d3 \\
\hline & C18:0 & Stearic & 27.2 & 2721 & $\mathrm{n} / \mathrm{a}$ & $\mathrm{n} / \mathrm{a}$ & quadratic & $1 / x^{2}$ & Stearic-d35 \\
\hline & C18:1n9 & Oleic & 89.5 & 8949 & $\mathrm{n} / \mathrm{a}$ & $\mathrm{n} / \mathrm{a}$ & linear & $1 / x^{2}$ & 13C-Oleic \\
\hline & C18:1n7 & cis-Vaccenic & 7.32 & 732 & $n / a$ & $\mathrm{n} / \mathrm{a}$ & quadratic & $1 / x^{2}$ & 13C-Oleic \\
\hline & $\mathrm{C} 18: 2 \mathrm{n} 6$ & Linoleic & 86.4 & 8635 & $\mathrm{n} / \mathrm{a}$ & $\mathrm{n} / \mathrm{a}$ & linear & $1 / x^{2}$ & 13C-Linoleic \\
\hline & C18:3n3 & alpha-Linolenic & 6.62 & 662 & $\mathrm{n} / \mathrm{a}$ & $\mathrm{n} / \mathrm{a}$ & linear & $1 / x^{2}$ & alpha-Linolenic-d14 \\
\hline & $\mathrm{C} 18: 3 \mathrm{n} 6$ & gamma-Linolenic & 2.99 & 299 & $\mathrm{n} / \mathrm{a}$ & $\mathrm{n} / \mathrm{a}$ & linear & $1 / x^{2}$ & alpha-Linolenic-d14 \\
\hline & C18:4n3 & Stearidonic & 0.49 & 50.3 & $\mathrm{n} / \mathrm{a}$ & $\mathrm{n} / \mathrm{a}$ & quadratic & $1 / x$ & Arachidic-d39 \\
\hline & $\mathrm{C} 20: 0$ & Arachidic & 0.95 & 95.1 & $\mathrm{n} / \mathrm{a}$ & $\mathrm{n} / \mathrm{a}$ & linear & $1 / x^{2}$ & Arachidic-d39 \\
\hline & C20:1n9 & 11-Eicosenoic & 1.02 & 102 & $\mathrm{n} / \mathrm{a}$ & $\mathrm{n} / \mathrm{a}$ & quadratic & $1 / x^{2}$ & Arachidic-d39 \\
\hline & $\mathrm{C} 20: 2 \mathrm{n} 6$ & 11,14-Eicosadienoic & 0.97 & 96.8 & $\mathrm{n} / \mathrm{a}$ & $\mathrm{n} / \mathrm{a}$ & quadratic & $1 / x^{2}$ & Arachidic-d39 \\
\hline & C20:3n9 & 5Z, 8Z, 11Z-Eicosatrienoic & 0.91 & 71.8 & $\mathrm{n} / \mathrm{a}$ & $\mathrm{n} / \mathrm{a}$ & quadratic & $1 / x^{2}$ & Arachidic-d39 \\
\hline & $\mathrm{C} 20: 3 \mathrm{n} 6$ & homo-gamma-Linolenic & 4.63 & 463 & $\mathrm{n} / \mathrm{a}$ & $\mathrm{n} / \mathrm{a}$ & quadratic & $1 / x^{2}$ & alpha-Linolenic-d14 \\
\hline & $\mathrm{C} 20: 4 \mathrm{n} 6$ & Arachidonic & 23.0 & 2300 & $\mathrm{n} / \mathrm{a}$ & $\mathrm{n} / \mathrm{a}$ & linear & $1 / x^{2}$ & Arachidonic-d8 \\
\hline & $C 20: 5 n 3$ & Eicosapentaenoic & 3.97 & 397 & $\mathrm{n} / \mathrm{a}$ & $n / a$ & quadratic & $1 / x^{2}$ & Eicosapentaenoic-d5 \\
\hline & C22:0 & Docosanoic & 1.94 & 194 & $\mathrm{n} / \mathrm{a}$ & $\mathrm{n} / \mathrm{a}$ & linear & $1 / x^{2}$ & Docosanoic-d4 \\
\hline & $\mathrm{C} 22: \ln 9$ & Docosenoic & 0.56 & 56.1 & $\mathrm{n} / \mathrm{a}$ & $\mathrm{n} / \mathrm{a}$ & quadratic & $1 / x$ & Docosanoic-d4 \\
\hline & $\mathrm{C} 22: 2 \mathrm{n} 6$ & 13,16- Docosadienoic & 0.50 & 45.5 & $n / a$ & $\mathrm{n} / \mathrm{a}$ & linear & $1 / x^{2}$ & Docosanoic-d4 \\
\hline & $\mathrm{C} 22: 4 \mathrm{n} 6$ & Docosatetraenoic & 1.55 & 155 & $n / a$ & $\mathrm{n} / \mathrm{a}$ & quadratic & $1 / x^{2}$ & Docosahexaenoic-d5 \\
\hline & $\mathrm{C} 22: 5 \mathrm{n} 3$ & Docosapentaenoic n-3 & 2.54 & 254 & $\mathrm{n} / \mathrm{a}$ & $\mathrm{n} / \mathrm{a}$ & linear & $1 / x^{2}$ & Docosahexaenoic-d5 \\
\hline & C22:5n6 & Docosapentaenoic n- 6 & 1.50 & 150 & $\mathrm{n} / \mathrm{a}$ & $\mathrm{n} / \mathrm{a}$ & linear & $1 / x^{2}$ & Docosahexaenoic-d5 \\
\hline & C22:6n3 & Docosahexaenoic & 8.06 & 806 & $\mathrm{n} / \mathrm{a}$ & $\mathrm{n} / \mathrm{a}$ & linear & $1 / x^{2}$ & Docosahexaenoic-d5 \\
\hline & C23:0 & Tricosanoic & 0.83 & 47.2 & $\mathrm{n} / \mathrm{a}$ & $\mathrm{n} / \mathrm{a}$ & linear & $1 / x^{2}$ & Docosanoic-d4 \\
\hline & C24:0 & Tetracosanoic & 1.40 & 140 & $\mathrm{n} / \mathrm{a}$ & $\mathrm{n} / \mathrm{a}$ & linear & $1 / x^{2}$ & Tetracosanoic-d4 \\
\hline & C24:1n9 & Nervonic & 1.99 & 199 & $\mathrm{n} / \mathrm{a}$ & $\mathrm{n} / \mathrm{a}$ & quadratic & $1 / x^{2}$ & Tetracosanoic-d4 \\
\hline & C26:0 & Hexacosanoic (cerotic) & 0.49 & 41.1 & $\mathrm{n} / \mathrm{a}$ & $\mathrm{n} / \mathrm{a}$ & linear & $1 / x^{2}$ & Tetracosanoic-d4 \\
\hline
\end{tabular}


Appendix B

Participants in Interlaboratory Analytical Comparison Study of Total Fatty Acid Concentrations in Human Serum Exercise 01: QA12FASER01 (in alphabetical order)

Centers for Disease Control and Prevention (CDC)

Nutritional Biomarkers Branch

4770 Buford Hwy, NE

Atlanta, GA 30341

Contact: Rosemary Schleicher and Carissa Powers

Cornell University,

Division of Nutritional Sciences

Savage Hall B38,

Ithaca, NY 14850

Contact: Tom Brenna and Peter Lawrence

Hanyang Univeristy

Dept of Food and Nutrition

College of Human Ecology

222 Wangsimni-ro

Seongdong-gu

Seoul 133-791, Korea

Contact: Yongsoon Park and Sunyoung Jo

Lipid Analytical Labs

University of Guelph Research Park

150 Research Lane, Room 100

Guelph, ON, Canada N1G 4T2

Contact: Jerry Piekarski and Bruce Holub

Mayo Clinic

Biochemical Genetics Laboratory Hilton 330

200 First Street S.W.

Rochester, MN 55905

Contact: Angela Tauscher 
Minnesota Department of Health

Public Health Laboratory Division

MDA/MDH Lab Building

601 Robert St. N.

Saint Paul, MN 55155-2531

Contact: Tsutomu Shimotori

National Institutes of Health

LMBB, DICBR, NIAAA

5625 Fishers Lane, Rm 3N01

Rockville, MD 20852

Contact: Yuhong Lin and Joseph Hibbeln

National Institute of Standards and Technology (NIST)

Chemical Sciences Division

100 Bureau Drive, MS 8392

Gaithersburg, MD 20899

Contact: Michele Schantz

OmegaQuant, LLC

2329 N. Career Ave, Suite 113

Sioux Falls, SD 57107

Contact: Jason Polreis

The University of Auckland

School of Biological Science

Room 110-285

3a Symonds Street

Auckland 1010 NEW ZEALAND

Contact: Dang-Dung Nguyen

University of Waterloo

Dept. Kinesiology

200 University Ave West

Waterloo, ON N2L 5W7 Canada

Contact: Ken Stark 